\title{
SYNTHESIS OF DENSELY FUNCTIONALIZED PYRIMIDOURACILS BY NICKEL(II)- CATALYZED ISOCYANIDE INSERTION
}

Jurriën W. Collet, ${ }^{\dagger,}, 1$ Bénédicte Morel, ${ }^{\ddagger}, 1$ Hung-Chien Lin, ${ }^{\dagger}$ Thomas R. Roose, ${ }^{\dagger}$ Pieter Mampuys, ${ }^{\ddagger}$ Romano V.A. Orru, ${ }^{\dagger}, *$ Eelco Ruijter ${ }^{\dagger, *}$ and Bert U.W. Maes ${ }^{* *}$

†Department of Chemistry and Pharmaceutical Sciences and Amsterdam Institute for Molecules, Medicines \& Systems (AIMMS), Vrije Universiteit Amsterdam, De Boelelaan 1108, 1081 HZ Amsterdam, The Netherlands. E-mail: e.ruijter@vu.nl; r.v.a.orru@vu.nl_http://syborch.com/ $\ddagger$ Organic Synthesis, Department of Chemistry, University of Antwerp, Groenenborgerlaan 171, 2020 Antwerp, Belgium

${ }^{1}$ These authors contributed equally to this work 


\section{Table of Contents}

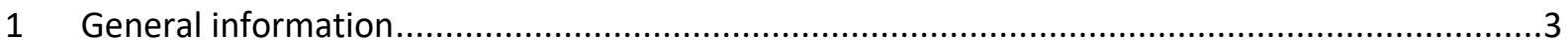

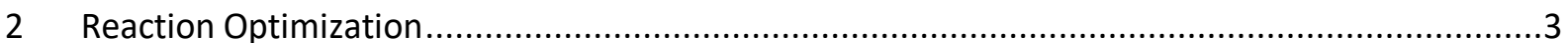

2.1 General procedure for the optimization of the direct oxidative imodoylative amination.......3

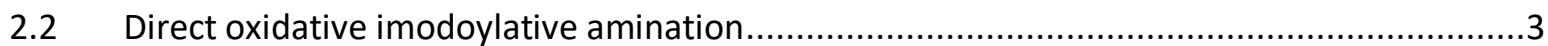

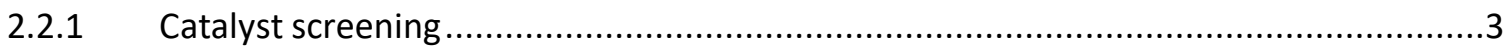

3 Mechanism .............................................................................. Error! Bookmark not defined.

3.1 Radical or ionic reaction ...................................................... Error! Bookmark not defined.

3.2 Influence of the oxidant........................................................ Error! Bookmark not defined.

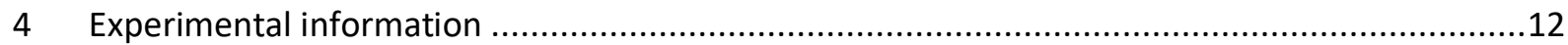

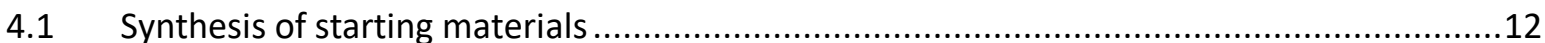

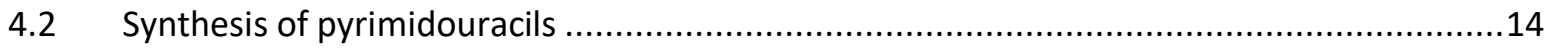

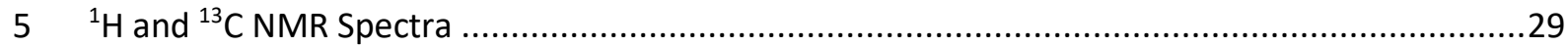

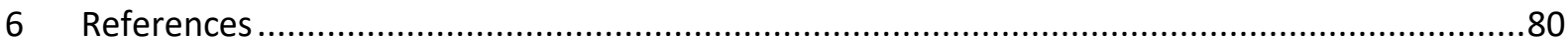




\section{General information}

Chemicals were purchased from Fischer Chemicals, TCl Chemicals or Sigma Aldrich and used without purification. Solvents were purchased from Fischer Chemicals (toluene, DCM, hexanes, EtOAc), or Acros Organics (DMSO, anisole) and used without purification, unless stated otherwise. NMR measurements were acquired either on a Bruker Avance 400 ( ${ }^{1} \mathrm{H}$ NMR: $400.13 \mathrm{MHz},{ }^{13} \mathrm{C}$ NMR: 100.61 $\mathrm{MHz}$, performed in $\mathrm{CDCl}_{3}$ (deuteration grade $>99.8 \%$, containing $\left.0.03(\mathrm{v} / \mathrm{v}) \% \mathrm{SiMe}_{4}\right)$ ) or a Bruker Avance 500.23 ( ${ }^{1} \mathrm{H}$ NMR: $500.23 \mathrm{MHz},{ }^{13} \mathrm{C}$ NMR: $125.78 \mathrm{MHz}$ (in APT mode)), performed in $\mathrm{CDCl}_{3}$ (deuteration grade $>99.8 \%)$ All shifts are reported in ppm downfield of tetramethylsilane, and are corrected according to solvent. $\mathrm{SiO}_{2}$ column chromatography was performed using Silicycle Siliaflash P60 Gel C60 (particle size 40-63 $\mu \mathrm{m}$ ) on a Teledyne CombiFlash Rf automated chromatography system with on-line UV detection, or by hand on Merck Silica Gel C60 (particle size: 40-60 $\mu \mathrm{m}$ ) using the indicated solvent system. TLC chromatography was performed on Merck Silica Gel C60 F254 plates (silica coat on aluminum support). HRMS was performed on an Ion Cyclotron Resonance (ICR) spectrometer with a positive ion polarity mode. All measured compounds displayed $<5.0 \mathrm{ppm}$ deviated from calculated values. All isolated yields are corrected for trace impurities, if present. Melting points were determined by a Büchi Melting Point M565 device and are uncorrected.

\section{Reaction Optimization}

\subsection{General procedure for the optimization of the direct oxidative imodoylative amination}

An oven-dried $10 \mathrm{~mL}$ pressure vial, equipped with a stir bar, was cooled down to room temperature under $\mathrm{O}_{2}$ atmosphere. Subsequently, it was charged with $\mathrm{N}$-(1,3-dibenzyl-2,6-dioxo-1,2,3,6tetrahydropyrimidin-4-yl)benzimidamide 1a ( $205 \mathrm{mg}, 0.5 \mathrm{mmol}, 1$ equiv), the catalyst and the solvent. The resulting mixture was stirred at room temperature under $\mathrm{O}_{2}\left(\operatorname{or} \mathrm{N}_{2}\right.$ ) atmosphere (balloon) prior to the addition of tert-butyl isocyanide $\mathbf{2 a}$. Subsequently, the reaction mixture was placed in an preheated oil bath and stirred for the indicated time under $\mathrm{O}_{2}$ (or $\mathrm{N}_{2}$ ) atmosphere (balloon). After cooling down, ethyl acetate $(60 \mathrm{~mL})$ and $\mathrm{NH}_{4} \mathrm{OH}$ (aq.)/saturated brine $(3: 2,20 \mathrm{~mL})$ were added to the reaction mixture. The organic layer was extracted, dried over $\mathrm{MgSO}_{4}$, filtered, concentrated under reduced pressure and thoroughly dried on a lyophilizer. 1,3,5-Trimethoxybenzene ( $84 \mathrm{mg}, 0.5 \mathrm{mmol}, 1$ equiv) was added to the crude reaction mixture, as internal standard, and everything was dissolved in DMSO$\mathrm{d}_{6}$. Quantitative ${ }^{1} \mathrm{H}$ NMR was performed based on manual integration of peaks belonging to starting material $\mathbf{1 a}$ and desired product $\mathbf{3 a}$ versus the internal standard.

\subsection{Direct oxidative imodoylative amination}

\subsubsection{Catalyst screening}

We started our studies to develop an intramolecular cross-dehydrogenative imidoylative amination towards functionalized pyrimidouracils with the model reaction of $\mathrm{N}-(1,3-$ dibenzyluracil)benzimidamide 1a with $t$-BuNC (2a). Remarkably, when applying the reaction conditions developed to obtain 1,3-dibenzyl-8-phenylxanthine from $\mathbf{1 a}$ by adding $\mathbf{2 a}$, a low conversion of $\mathbf{1 a}$ and no formation of the desired compound $3 a$ was obtained (Table S1, entry 1). Various $\mathrm{Cu}(\mathrm{I})$ and $\mathrm{Cu}(\mathrm{II})$ catalysts were subsequently evaluated, but they all failed to facilitate the imidoylative coupling of 1a with $\mathbf{2 a}$ (Table S1, entry 1-4). In addition, iron salts also failed to deliver the desired product (entries 5 and 6). Zhu et al. ${ }^{[1]}$ reported the imidoylative cycloaromatization of $\mathrm{N}$-arylamidines to give 4aminoquinazolines under palladium catalysis. We therefore also examined $\mathrm{Pd}(\mathrm{OAc})_{2}$ as catalyst for this transformation and 3a was obtained in $54 \%$ yield (entry 7). Satisfyingly, several nickel and cobalt catalysts also facilitated the oxidative coupling (Table $\mathrm{S} 1$, entries $8-13$ ). $\mathrm{Ni}(\mathrm{OAc})_{2} \cdot 4 \mathrm{H}_{2} \mathrm{O}$ was selected as 
optimal catalyst for further optimization at $120{ }^{\circ} \mathrm{C}$ as pyrimidouracil $3 a$ was obtained in $93 \%$ after $5 \mathrm{~h}$ (Table S1, entry 10).

Table S1. Catalyst screening for imidoylative cycloamination towards $3{ }^{a}{ }^{a}$<smiles>CC(C)(C)NC(=O)c1ccccc1</smiles><smiles>CC(C)(C)Nc1nc(-c2ccccc2)nc2c1c(=O)n(Cc1ccccc1)c(=O)n2Cc1ccccc1</smiles>

$1 a$

$2 a$

3a

\begin{tabular}{llll}
\hline entry & Catalyst & Conversion of 1a $(\%) b$ & yield 3a $(\%) b$ \\
1 & $\mathrm{CuI}$ & 14 & - \\
$2 \mathrm{c}$ & $\mathrm{CuI}$ & 12 & - \\
3 & $\mathrm{CuOAc}$ & 28 & - \\
4 & $\mathrm{Cu}(\mathrm{OAc})_{2} \cdot \mathrm{H}_{2} \mathrm{O}$ & 13 & - \\
5 & $\mathrm{Fe}(\mathrm{OAc})_{2}$ & 15 & - \\
5 & $\mathrm{Fe}_{2}\left(\mathrm{SO}_{4}\right)_{3} \cdot 5 \mathrm{H}_{2} \mathrm{O}$ & 12 & - \\
$7 \mathrm{Pd}, \mathrm{e}$ & $\mathrm{Pd}(\mathrm{OAc})_{2}$ & n.d. & 54 \\
8 & $\mathrm{Ni(acac})_{2}$ & 52 & 41 \\
9 & $\mathrm{Ni}(\mathrm{OTf})_{2}$ & 15 & - \\
10 & $\mathrm{Ni}(\mathrm{OAc})_{2} \cdot 4 \mathrm{H}_{2} \mathrm{O}$ & 100 & 93 \\
11 & $\mathrm{Ni}(\mathrm{cod})_{2}$ & 88 & 88 \\
12 & $\mathrm{Co}(\mathrm{OAc})_{2} \cdot 4 \mathrm{H}_{2} \mathrm{O}$ & 70 & 62 \\
13 & $\mathrm{Co}\left(\mathrm{SO}_{4}\right)_{2} \cdot 7 \mathrm{H}_{2} \mathrm{O}$ & 19 & -
\end{tabular}

a Reaction conditions: amidinouracil (1a, $205 \mathrm{mg}, 0.5 \mathrm{mmol}, 1$ equiv), tert-butyl isocyanide (2a, $169 \mu \mathrm{L}, 1.5$ $\mathrm{mmol}, 3.0$ equiv), catalyst ( $15 \mathrm{~mol} \%)$, DMSO $(2 \mathrm{~mL})$ were stirred at $120^{\circ} \mathrm{C}$ for $5 \mathrm{~h}$ under $\mathrm{O}_{2}$ atmosphere $(1$ bar, balloon). ${ }^{b}$ Determined by ${ }^{1} \mathrm{H}$ NMR analysis using 1,3,5-trimethoxybenzene as internal standard. ${ }^{\mathrm{c}}$ Reaction performed employing $t-\mathrm{Bu}_{2} \mathrm{O}_{2}$ as a stoichiometric oxidant. ${ }^{\mathrm{d}} \mathrm{Pd}(\mathrm{OAc})_{2}(5 \mathrm{~mol} \%)$ was used. e Reaction performed in DMF with $\mathrm{Cs}_{2} \mathrm{CO}_{3}(326 \mathrm{mg}, 2.0$ equiv) as base. N.D. $=$ not determined.

Optimization of the temperature and the reaction time indicated that the reaction could be performed at much lower temperatures, without negative effects on the yield of 3a (Table S2, entry 1-4) Attempts to lower the reaction temperature to $40^{\circ} \mathrm{C}$ or rt resulted in incomplete conversion (entry 4-6)

Table S2. Optimization of temperature and time for direct oxidative imidoylative amination towards $3 \mathrm{a}^{\mathrm{a}}$

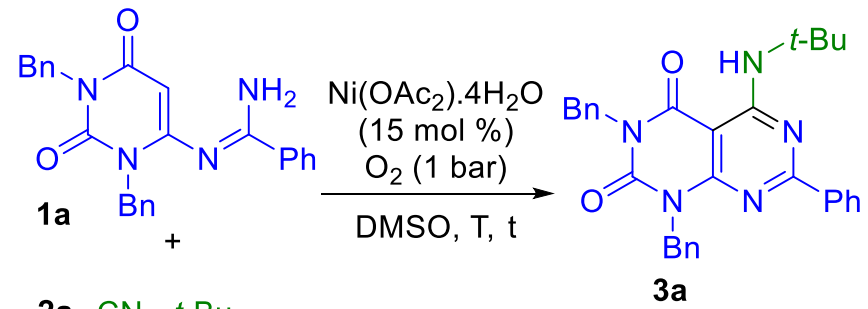

2a $\mathrm{CN}-t-\mathrm{Bu}$

\begin{tabular}{cccccc}
\hline entry & $\mathrm{T}\left({ }^{\circ} \mathrm{C}\right)$ & $\mathrm{t}(\mathrm{h})$ & recoup 1a $(\%)$ & Yield 3a $(\%)^{\mathrm{b}}$ & Mass balance \\
\hline & 120 & 5 & 0 & 93 & 93 \\
2 & 80 & 5 & 0 & 95 & 95 \\
3 & $\mathbf{6 o}$ & $\mathbf{5}$ & $\mathbf{0}$ & $\mathbf{9 4}$ & 94 \\
4 & 40 & 5 & 5 & 94 & 99 \\
5 & 40 & 18 & 0 & 90 & 90 \\
6 & 25 & 18 & 20 & 68 & 88
\end{tabular}

a Reaction conditions: 1a (o.5 mmol), $\mathbf{2 a}\left(3\right.$ eq.), $\mathrm{Ni}(\mathrm{OAc})_{2} \cdot 4 \mathrm{H}_{2} \mathrm{O}(15 \mathrm{~mol} \%)$, DMSO (o.25M), temperature (oil bath), $\mathrm{O}_{2}$ atmosphere (balloon), reaction time (h). ${ }^{b} \mathrm{NMR}$ yield (1,3,5-trimethoxybenzene as internal standard). 
Attempts to lower the catalyst loading to $10 \mathrm{~mol} \%$ (Table S3, entry 1-2) did not lead to a significant lowering of the yield of 3a. Catalyst loadings of $5 \mathrm{~mol} \%$ (Table S3, entry 3-5) were found effective at a temperature of $50^{\circ} \mathrm{C}$ (entry 4).

Table S3. Optimization of catalyst loading and temperature for direct oxidative imidoylative amination towards $3 a{ }^{a}$

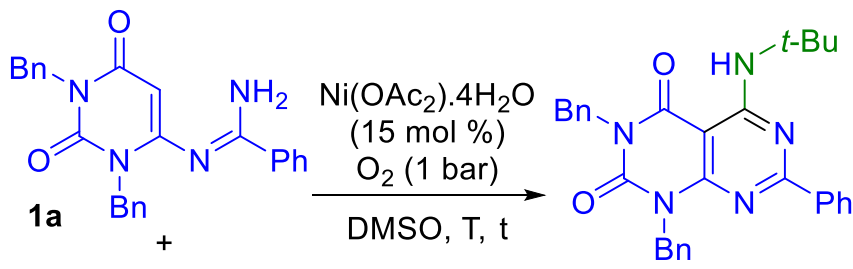

2a $\mathrm{CN}-\mathrm{t}-\mathrm{Bu}$

$3 a$

$\begin{array}{ccccccc}\text { entry } & \begin{array}{c}\text { Catalyst loading } \\ (\text { mol \%) }\end{array} & \mathrm{T}\left({ }^{\circ} \mathrm{C}\right) & \mathrm{t}(\mathrm{h}) & \text { recoup 1a (\%) } & \text { Yield 3a (\%) } & \text { Mass balance } \\ \mathbf{1} & 10 & 60 & 5 & 0 & 98 & 98 \\ 2 & 10 & 40 & 18 & 1 & 92 & 93 \\ 3 & 5 & 60 & 18 & 0 & 93 & 93 \\ 4 & 5 & 50 & \mathbf{1 8} & \mathbf{0} & 97 & 97 \\ 5 & 5 & 40 & \mathbf{1 8} & 5 & 91 & 96\end{array}$

a Reaction conditions: $\mathbf{1 a}(\mathrm{o} .5 \mathrm{mmol})$, $\mathbf{2 a}\left(3\right.$ eq.), $\mathrm{Ni}(\mathrm{OAc})_{2} \cdot 4 \mathrm{H}_{2} \mathrm{O}(15 \mathrm{~mol} \%)$, DMSO (o.25M), temperature (oil bath), $\mathrm{O}_{2}$ atmosphere (balloon), reaction time (h). ${ }^{b}$ NMR yield (1,3,5-trimethoxybenzene as internal standard).

In keeping with the CHEM21 solvent classification ${ }^{[2]}$, greener solvents than DMSO were screened. Satisfyingly, the reaction was afforded the target 3a in most solvents (Table S4). At $50{ }^{\circ} \mathrm{C}$, near full conversion to the product 3a was observed in apolar solvents (entries 4-8). Protic ethylene glycol (entry 3) did not afford any product 3a. As such, anisole was selected as the optimal solvent, due to its effectiveness of mediating this reaction, combined with its recommended status, and high flash point.

Table S4. Optimization of conditions for direct oxidative imidoylative amination towards $3{ }^{a}{ }^{a}$

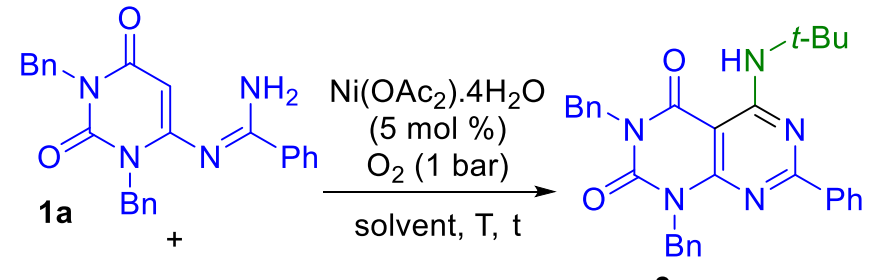

2a $\mathrm{CN}-\mathrm{t}-\mathrm{Bu}$

$3 a$

\begin{tabular}{|c|c|c|c|c|c|}
\hline entry & Solventb & $\mathrm{T}\left({ }^{\circ} \mathrm{C}\right)$ & $\mathrm{t}(\mathrm{h})$ & $\begin{array}{c}\text { Conversion of } 1 \mathrm{a} \\
(\%)\end{array}$ & Yield za (\%)c \\
\hline 1 & DMSO & 50 & 18 & 100 & 97 \\
\hline 2 & $\mathrm{CHCl}_{3}$ & 50 & 16 & 67 & 66 \\
\hline 3 & EG & 50 & 48 & 24 & - \\
\hline 4 & MIBK & 50 & 48 & 98 & 95 \\
\hline 5 & $\mathrm{BuOAc}$ & 50 & 48 & 95 & 93 \\
\hline 6 & Anisole & 50 & 16 & 100 & 99 \\
\hline 7 & DMC & 50 & 48 & 100 & 94 \\
\hline 8 & PC & 50 & 48 & 100 & 95 \\
\hline
\end{tabular}

a Reaction conditions: $\mathrm{N}$-(1,3-dibenzyluracil)benzimidamide (1a, $0.5 \mathrm{mmol}$, 1 equiv), tert-butyl isocyanide (2a, $1.25 \mathrm{mmol}, 2.5$ equiv), and $\mathrm{Ni}(\mathrm{OAc})_{2} \cdot 4 \mathrm{H}_{2} \mathrm{O}$, (0.025 mmol, $5 \mathrm{~mol} \%$ ) were stirred at indicated temperature for indicated time. ${ }^{b}$ Colored according to Chem21 solvent guide ${ }^{[2]}$ ( $\mathrm{red}=$ hazardous, yellow $=$ problematic, green $=$ recommended) ${ }^{c}$ Yield determined by ${ }^{1} \mathrm{H}$ NMR analysis using 1,3,5-trimethoxybenzene as internal standard. 
performed under air atm. EG = ethylene glycol, $\mathrm{MIBK}=$ methyl isobutyl ketone, $\mathrm{DMC}=$ dimethyl carbonate, $\mathrm{PC}=$ propylene carbonate.

Hereafter, we investigated the required atmosphere for this reaction, results imply that the reaction runs to completion within several hours, under both $\mathrm{O}_{2}$ (Table S5, entry 1 ), and air atmosphere. (entry 2-8). Although the insertion of tert-butyl isocyanide $\mathbf{2 a}$ affords the product $\mathbf{3 a}$ in near quantitative yields both at $1 \mathrm{~mol} \%$ catalyst loading (entry 2), and after 2 hours (entry 6), both these parameters are specific for isocyanide 2a. Utilization of other, less sterically hindered isocyanides lead to incomplete conversion after 2 hours, or at $1 \mathrm{~mol} \% \mathrm{Ni}(\mathrm{OAc})_{2} \cdot 4 \mathrm{H}_{2} \mathrm{O}$. As such, we opted for 16 hours reaction time, and a catalyst loading of $5 \mathrm{~mol} \%$ as the optimal, broadly applicable conditions (entry 8 ).

Table S5. Catalyst loading, atmosphere and time optimization ${ }^{[a]}$<smiles>N/C(=N\c1cc(=O)n(Cc2ccccc2)c(=O)n1Cc1ccccc1)c1ccccc1</smiles>

$1 \mathrm{a}$

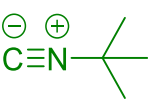

$2 \mathrm{a}$

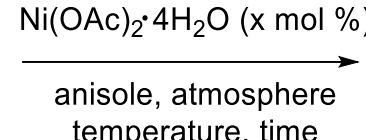
temperature, time<smiles>CC(C)(C)Nc1nc(-c2ccccc2)nc2c1c(=O)n(Cc1ccccc1)c(=O)n2Cc1ccccc1</smiles>

$3 a$

(2.5 equiv)

\begin{tabular}{|c|c|c|c|c|c|c|c|}
\hline Entry & $\begin{array}{l}\text { Reaction } \\
\text { number }\end{array}$ & Atm & $\begin{array}{l}\text { Catalyst loading } \\
\text { (mol \%) }\end{array}$ & $\begin{array}{c}\mathrm{t} \\
(\mathrm{h})\end{array}$ & $\begin{array}{l}3 \mathrm{a} \\
(\%)^{\mathrm{b}}\end{array}$ & $\begin{array}{c}\text { Recoup ra } \\
(\%)^{b}\end{array}$ & $\begin{array}{c}\text { Mass Balance } \\
(\%)^{\mathrm{b}}\end{array}$ \\
\hline 1 & $720 \mathrm{~A}$ & $\mathrm{O}_{2}$ & 5 & 16 & 100 & o & 100 \\
\hline 2 & 727 & air & 1 & 16 & 99 & o & 99 \\
\hline 3 & $725 \mathrm{~A}$ & air & 5 & o & 6 & 104 & 110 \\
\hline 4 & $725 \mathrm{~B}$ & air & 5 & 0.5 & 67 & 40 & 107 \\
\hline 5 & ${ }_{725} \mathrm{C}$ & air & 5 & 1 & 75 & 30 & 105 \\
\hline 6 & $725 \mathrm{D}$ & air & 5 & 2 & 100 & o & 100 \\
\hline 7 & $725 \mathrm{E}$ & air & 5 & 3 & 101 & o & 101 \\
\hline 8 & $723 A$ & air & 5 & 16 & 100 & $\mathbf{o}$ & 100 \\
\hline
\end{tabular}

a Reaction conditions: $\mathrm{N}$-(1,3-dibenzyluracil)benzimidamide (1a, $205 \mathrm{mg}, 0.5 \mathrm{mmol}$, 1 equiv), tert-butyl isocyanide (2a, $141 \mu \mathrm{L}, 1.25 \mathrm{mmol}, 2.5$ equiv), $\mathrm{Ni}(\mathrm{OAc})_{2} \cdot 4 \mathrm{H}_{2} \mathrm{O}$ ( $\mathrm{x} \mathrm{mol} \%$ ), anisole $(2 \mathrm{~mL})$ were stirred at indicated time and temperature. ${ }^{b}$ Yield determined by ${ }^{1} \mathrm{H}$ NMR analysis using $1,3,5$-trimethoxybenzene as internal standard. 


\section{Oxidant influence}

To our surprise, in the absence of an atmospheric oxidant 1,3-dibenzyl-5-(tert-butylamino)-7phenylpyrimido[4,5- $d$ ] pyrimidine-2,4(1H,3H)-dione (3a) was still obtained in $42 \%$ yield (Table 56 , entry 1). This result prompted further investigation. Direct quenching of the reaction mixture with $2 \mathrm{M}$ hydrochloric acid $(5 \mathrm{~mL})$, prior to ${ }^{1} \mathrm{H}$ NMR analysis, afforded the product $3 a$ in a similar yield, indicating that the reaction does not run to completion within the NMR tube. Vigorous degassing of all liquid reactants and solvents, via one or three freeze-pump-thaw cycles, did not hamper the conversion of $\mathrm{N}$-(1,3-dimethyluracil)benzimidamide 1a (entry 3-4), indicating oxygen is not responsible for the reoxidation of the nickel-catalyst.

Table S6. Reaction under argon atmosphere. ${ }^{a}$<smiles>N/C(=N\c1cc(=O)n(Cc2ccccc2)c(=O)n1Cc1ccccc1)c1ccccc1</smiles>

$1 \mathrm{a}$

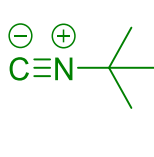

2a

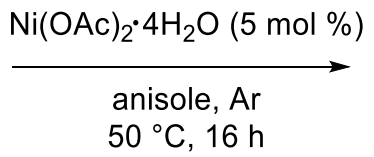
$50{ }^{\circ} \mathrm{C}, 16 \mathrm{~h}$<smiles>CC(C)(C)Nc1nc(-c2ccccc2)nc2c1c(=O)n(Cc1ccccc1)c(=O)n2Cc1ccccc1</smiles>

3a

(2.5 equiv)

$\begin{array}{llllll}\text { Entry } & \begin{array}{l}\text { Reaction } \\ \text { number }\end{array} & \text { Comments } & \text { 3a }(\%)^{\mathrm{b}} & \text { Recoup 1a (\%) }^{\mathrm{b}} & {\text { Mass Balance }(\%)^{\mathrm{b}}}^{\text {R }} \\ 1 & \text { JC724A } & - & 42 & 60 & 102 \\ 2 & \text { JC726B } & \text { Quenching with HCl (2 M) } & 47 & 46 & 93 \\ 3 & \text { JC724B } & \text { 1 Freeze-Pump-thaw cycle } & 34 & 64 & 98 \\ 4 & \text { JC724C } & \text { 3 Freeze-Pump-thaw cycles } & 58 & 44 & 102\end{array}$

a Reaction conditions: $N$-(1,3-dibenzyluracil)benzimidamide (1a, $205 \mathrm{mg}, 0.5 \mathrm{mmol}, 1$ equiv), tert-butyl isocyanide (2a, 2.5 equiv), $\mathrm{Ni}(\mathrm{OAc})_{2} \bullet 4 \mathrm{H}_{2} \mathrm{O}$ (0.006 g, $0.025 \mathrm{mmol}, 5 \mathrm{~mol} \%$ ), anisole ( $2 \mathrm{~mL}, 0.25 \mathrm{M}$ ) were stirred at $50{ }^{\circ} \mathrm{C}$ for $16 \mathrm{~h}$ under Ar atmosphere (1 bar, balloon). ${ }^{b}$ Yield determined by ${ }^{1} \mathrm{H}$ NMR analysis using ${ }^{1,3,5^{-}}$ trimethoxybenzene as internal standard.

Hereafter, we varied the equivalents of tert-butyl isocyanide (2a), with both normal (Table S7Error! Reference source not found., entries 1-4) and high (Table S7, entry 5-8) catalyst loading (see also figure S1). Interestingly, the yield of $3 a$ is decreased in the presence of a higher amount of $\mathrm{Ni}(\mathrm{OAc})_{2} \cdot 4 \mathrm{H}_{2} \mathrm{O}$. The significant difference between catalyst loadings implies the reaction requires outer sphere addition of an external isocyanide moiety. In absence hereof, the catalysis apparently slows at the stable $\mathrm{Ni}(t-\mathrm{BuNC})_{\times}(\mathrm{OAc})_{2}$ species.

Table S7. Ratio 1a/2a and catalyst loading screening. ${ }^{a}$<smiles>N/C(=N\c1cc(=O)n(Cc2ccccc2)c(=O)n1Cc1ccccc1)c1ccccc1</smiles>

$1 \mathrm{a}$

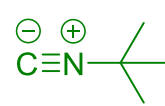

2a<smiles>CC(C)(C)Nc1nc(-c2ccccc2)nc2c1c(=O)n(Cc1ccccc1)c(=O)n2Cc1ccccc1</smiles>

3a

\begin{tabular}{ccccccc} 
Entry & $\begin{array}{c}\text { Reaction } \\
\text { number }\end{array}$ & $\begin{array}{c}\mathbf{2 a} \\
\text { (equiv) }\end{array}$ & $\begin{array}{c}\text { Catalyst loading } \\
(\text { mol \%) }\end{array}$ & $\begin{array}{c}\text { 3a } \\
(\%)^{\mathrm{b}}\end{array}$ & $\begin{array}{c}\mathbf{1 a} \\
(\%)^{\mathrm{b}}\end{array}$ & $\begin{array}{c}\text { Mass Balance } \\
(\%)^{\mathrm{b}}\end{array}$ \\
\hline 1 & JC737A & 1.00 & 5 & 76 & 26 & 102 \\
2 & JC737B & 0.75 & 5 & 56 & 45 & 101 \\
\hline
\end{tabular}




$\begin{array}{lllllll}3 & \text { JC737C } & 0.50 & 5 & 48 & 55 & 103 \\ 4 & \text { JC737D } & 0.25 & 5 & 21 & 84 & 105 \\ 5 & \text { JC773A } & 1.00 & 25 & 55 & 42 & 97 \\ 6 & \text { JC773B } & 0.75 & 25 & 46 & 54 & 100 \\ 7 & \text { JC773C } & 0.50 & 25 & 28 & 80 & 108 \\ 8 & \text { JC773D } & 0.25 & 25 & 19 & 84 & 103\end{array}$

a Reaction conditions: $\mathrm{N}$-(1,3-dibenzyluracil)benzimidamide (1a, $205 \mathrm{mg}, 0.5 \mathrm{mmol}$, 1 equiv), tert-butyl isocyanide (2a, 2.5 equiv), $\mathrm{Ni}(\mathrm{OAc})_{2} \bullet 4 \mathrm{H}_{2} \mathrm{O}(5 \mathrm{~mol} \%)$, anisole $(2 \mathrm{~mL}, 0.25 \mathrm{M})$ were stirred at $50{ }^{\circ} \mathrm{C}$ for 70 h under Ar atmosphere (1 bar, balloon). ${ }^{b}$ Yield determined by ${ }^{1} \mathrm{H}$ NMR analysis using 1,3,5-trimethoxybenzene as internal standard.

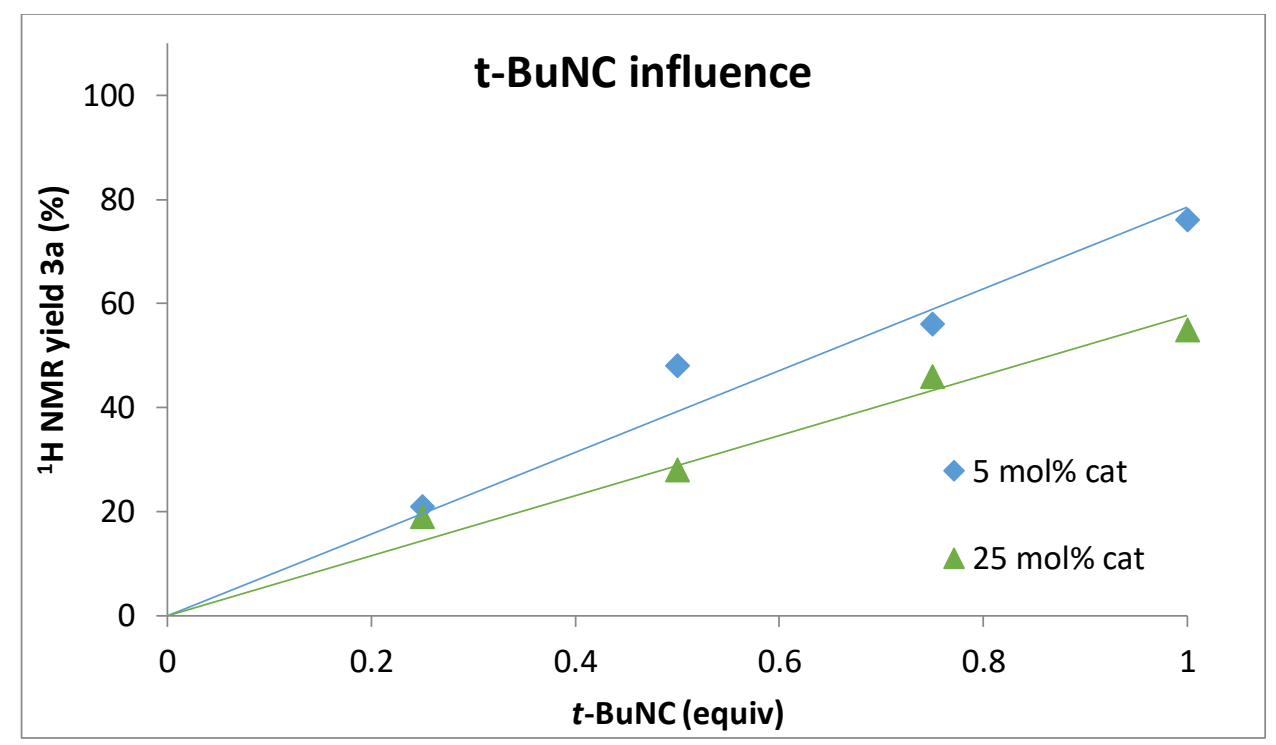

Figure S1. Investigations into maximum yield, limited by za concentration

Additionally, atmospheric influence was investigated to confirm a dehydrogenative version of the reaction in Table S8. Interestingly, we did observe a significantly slower consumption of reactant 1a in the presence of a hydrogen atmosphere, implying the reaction indeed proceeds dehydrogenatively in the absence of a suitable oxidant (Table S8, Figure S2). Hereafter, we investigated the possibility of adding a hydrogen acceptor to the reaction mixture to confirm these findings, but neither styrene nor benzoquinone were suitable hydrogen acceptors, and while the product $\mathbf{3 a}$ was formed under these conditions, no by-product ethylbenzene or hydroquinone was observed (Table S9).

Table S8. Evaluation of the reaction atmosphere and time.<smiles>N/C(=N\c1cc(=O)n(Cc2ccccc2)c(=O)n1Cc1ccccc1)c1ccccc1</smiles>

$1 \mathrm{a}$

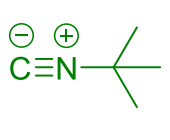

2a

$\begin{array}{ll}\text { Entry } & \text { Reaction number } \\ 1 & \text { JC892A } \\ 2 & \text { JC892B } \\ 3 & \text { JC892C }\end{array}$

$\mathrm{Ni}(\mathrm{OAc})_{2} 4 \mathrm{H}_{2} \mathrm{O}(5 \mathrm{~mol} \%)$

anisole, atmosphere

$50{ }^{\circ} \mathrm{C}$, time<smiles>CC(C)(C)Nc1nc(-c2ccccc2)nc2c1c(=O)n(Cc1ccccc1)c(=O)n2Cc1ccccc1</smiles>

3a

$\begin{array}{llll}\text { Atmosphere } & \text { time }(\mathrm{h}) & \text { 3a }(\%)^{\mathrm{b}} & \text { Recoup 1a (\%) } \\ \mathrm{N}_{2} & 0 & 0 & 100 \\ \mathrm{~N}_{2} & 1.5 & 44 & 56 \\ \mathrm{~N}_{2} & 2.5 & 50 & 50\end{array}$




$\begin{array}{llllll}4 & \text { JC892D } & \mathrm{N}_{2} & 3.5 & 53 & 47 \\ 5 & \text { JC892A } & \mathrm{H}_{2} & 0 & 0 & 100 \\ 6 & \text { JC892B } & \mathrm{H}_{2} & 1.5 & 9 & 91 \\ 7 & \text { JC892C } & \mathrm{H}_{2} & 2.5 & 29 & 71 \\ 8 & \text { JC892D } & \mathrm{H}_{2} & 3.5 & 37 & 63\end{array}$

a Reaction conditions: $N$-(1,3-dibenzyluracil)benzimidamide (1a, $205 \mathrm{mg}, 0.5 \mathrm{mmol}, 1$ equiv), tert-butyl isocyanide (2a, $139 \mu \mathrm{L}, 2.5$ equiv), $\mathrm{Ni}(\mathrm{OAc})_{2} \bullet 4 \mathrm{H}_{2} \mathrm{O}(5 \mathrm{~mol} \%)$, anisole $(2 \mathrm{~mL}, 0.25 \mathrm{M})$ were stirred at $5 \mathrm{O}^{\circ} \mathrm{C}$ for indicated time under indicated atmosphere (1 bar, balloon). ${ }^{\mathrm{b}}$ Yield determined by ${ }^{1} \mathrm{H}$ NMR analysis using $1,3,5^{-}$ trimethoxybenzene as internal standard.

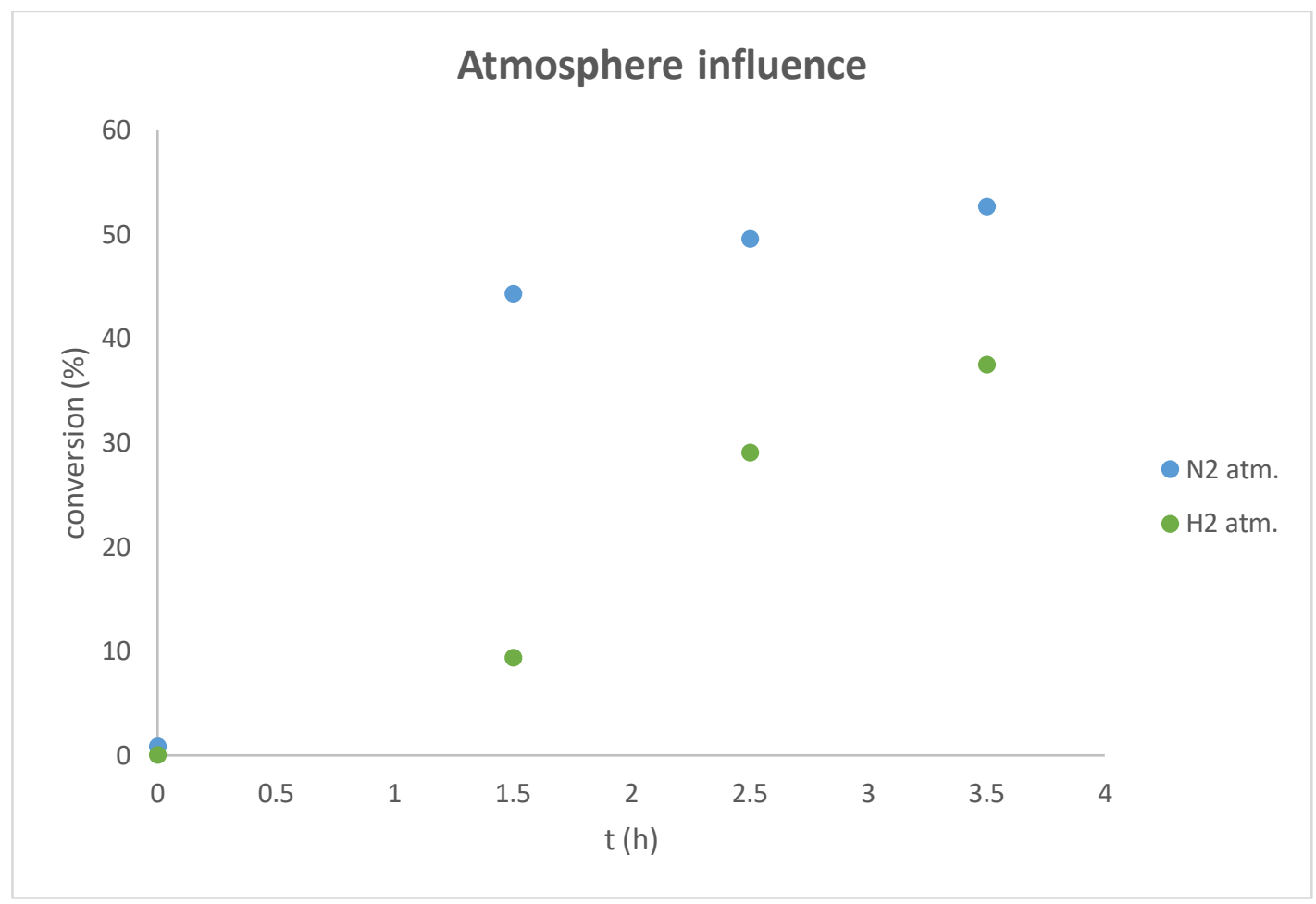

Figure S2. Evaluation of the reaction atmosphere

Table S9. Hydrogen trapping experiments<smiles>N/C(=N\c1cc(=O)n(Cc2ccccc2)c(=O)n1Cc1ccccc1)c1ccccc1</smiles>

$1 \mathrm{a}$
$\mathrm{Ni}(\mathrm{OAc})_{2} 4 \mathrm{H}_{2} \mathrm{O}(5 \mathrm{~mol} \%)$ additive

anisole, argon

$50{ }^{\circ} \mathrm{C}, 16 \mathrm{~h}$<smiles>CC(C)(C)Nc1nc(-c2ccccc2)nc2c1c(=O)n(Cc1ccccc1)c(=O)n2Cc1ccccc1</smiles>

$3 a$

\begin{tabular}{llllll} 
Entry & $\begin{array}{l}\text { Reaction } \\
\text { number }\end{array}$ & Additive (equiv) & 3a (\%) & Recoup 1a (\%) & Mass Balance (\%) \\
\hline 1 & JC932 & Styrene (5.0) & 51 & 50 & 100 \\
2 & JC735A & benzoquinone (2.0) & 44 & 56 & 100
\end{tabular}

a Reaction conditions: $\mathrm{N}$-(1,3-dibenzyluracil)benzimidamide (1a, $205 \mathrm{mg}, 0.5 \mathrm{mmol}, 1$ equiv), tert-butyl isocyanide (2a, $139 \mu \mathrm{L}, 2.5$ equiv), additive, $\mathrm{Ni}(\mathrm{OAc})_{2} \bullet 4 \mathrm{H}_{2} \mathrm{O}(5 \mathrm{~mol} \%)$, anisole $(2 \mathrm{~mL}, 0.25 \mathrm{M})$ were stirred at 50 ${ }^{\circ} \mathrm{C}$ for $16 \mathrm{~h}$ under argon atmosphere (1 bar, balloon). ${ }^{\mathrm{b}}$ Yield determined by ${ }^{1} \mathrm{H}$ NMR analysis using $1,3,5-$ trimethoxybenzene as internal standard. 


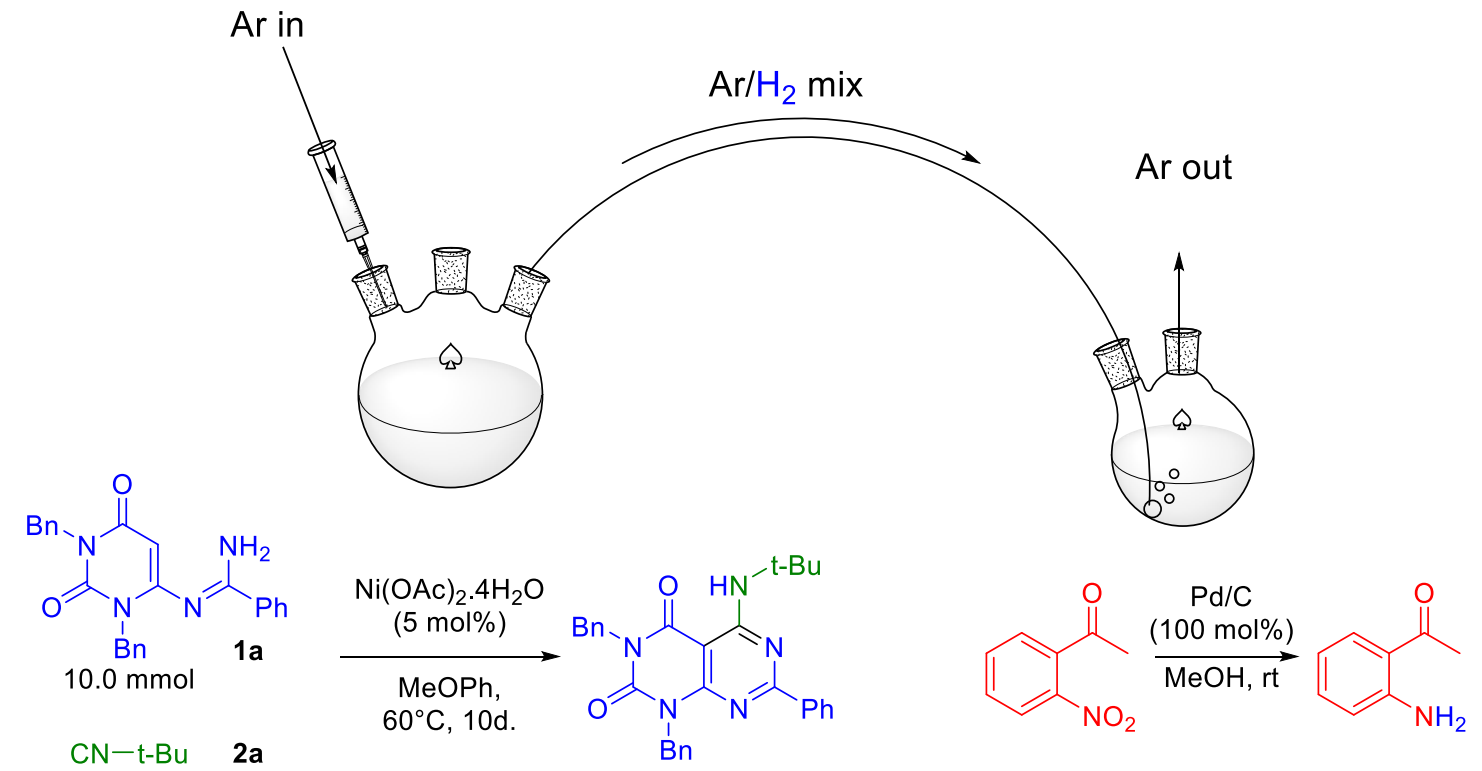

Scheme $S$ 1. Hydrogenation experiment to indicate dehydrogenative coupling.

Subsequently, we diverted the argon headspace of the nickel catalyzed isocyanide insertion through a palladium catalyzed hydrogenation (Scheme S2). For this hydrogenation experiment, we opted for 0 nitroacetophenone as substrate, since the hydrogenated analogue $o$-aminoacetophenone is highly fluorescent, and therefore more readily detectable. Additionally, the hydrogenation of nitroarenes is among the most readily facilitated hydrogenations. Running the imidoylation to completion on a 10 $\mathrm{mmol}$ scale, and directing the headspace through a suspension of palladium on activated carbon, allowed for the isolation of a small fluorescent spot with the same $R_{f}$ as $O$-aminoacetophenone. The ${ }^{1} \mathrm{H}$ NMR shows the formation of two new compounds, of which one directly corresponds to $O$ aminoacetophenone (See NMR analysis, Figure S3). The other appears to be 0 hydroxylaminoacetophenone., of which the presence was confirmed by HRMS. A negative control with identical reaction conditions, without the addition of $\mathrm{Ni}(\mathrm{OAc})_{2} \cdot 4 \mathrm{H}_{2} \mathrm{O}$ as a catalyst did not lead to any of the aforementioned results. Only reactant $1 \mathrm{a}$ and the $o$-nitroacetophenone were observed.

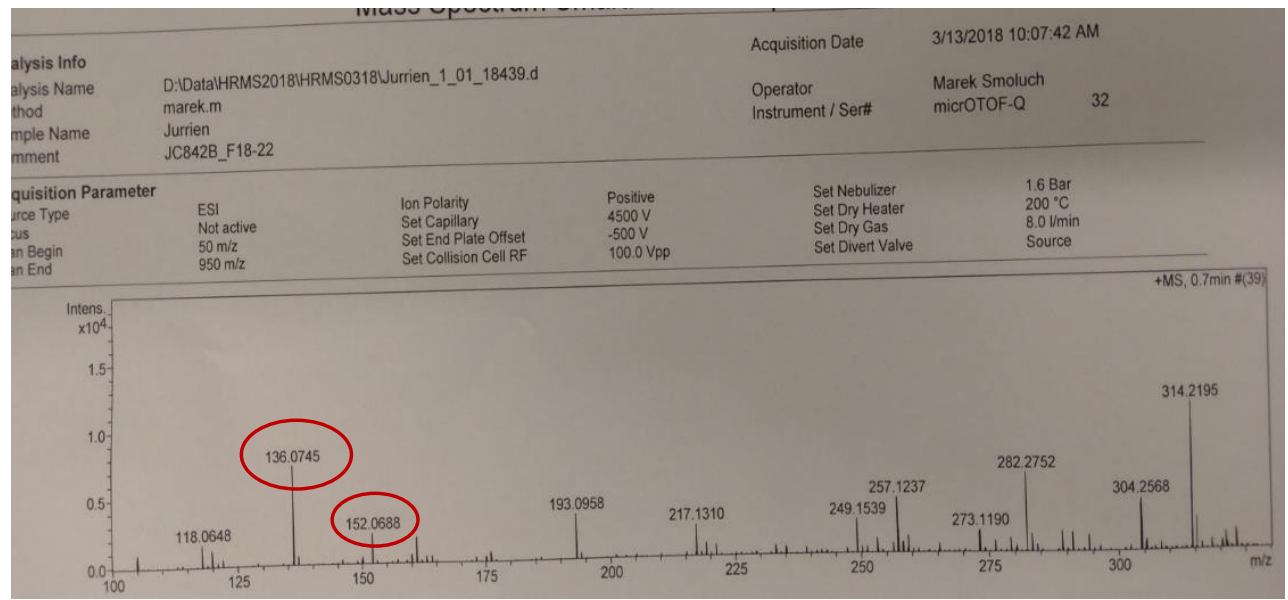

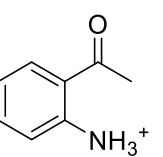

calculated mass 136.0757

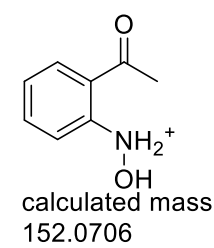

Figure $S_{4}$. HRMS confirmation of hydrogenolysis products 

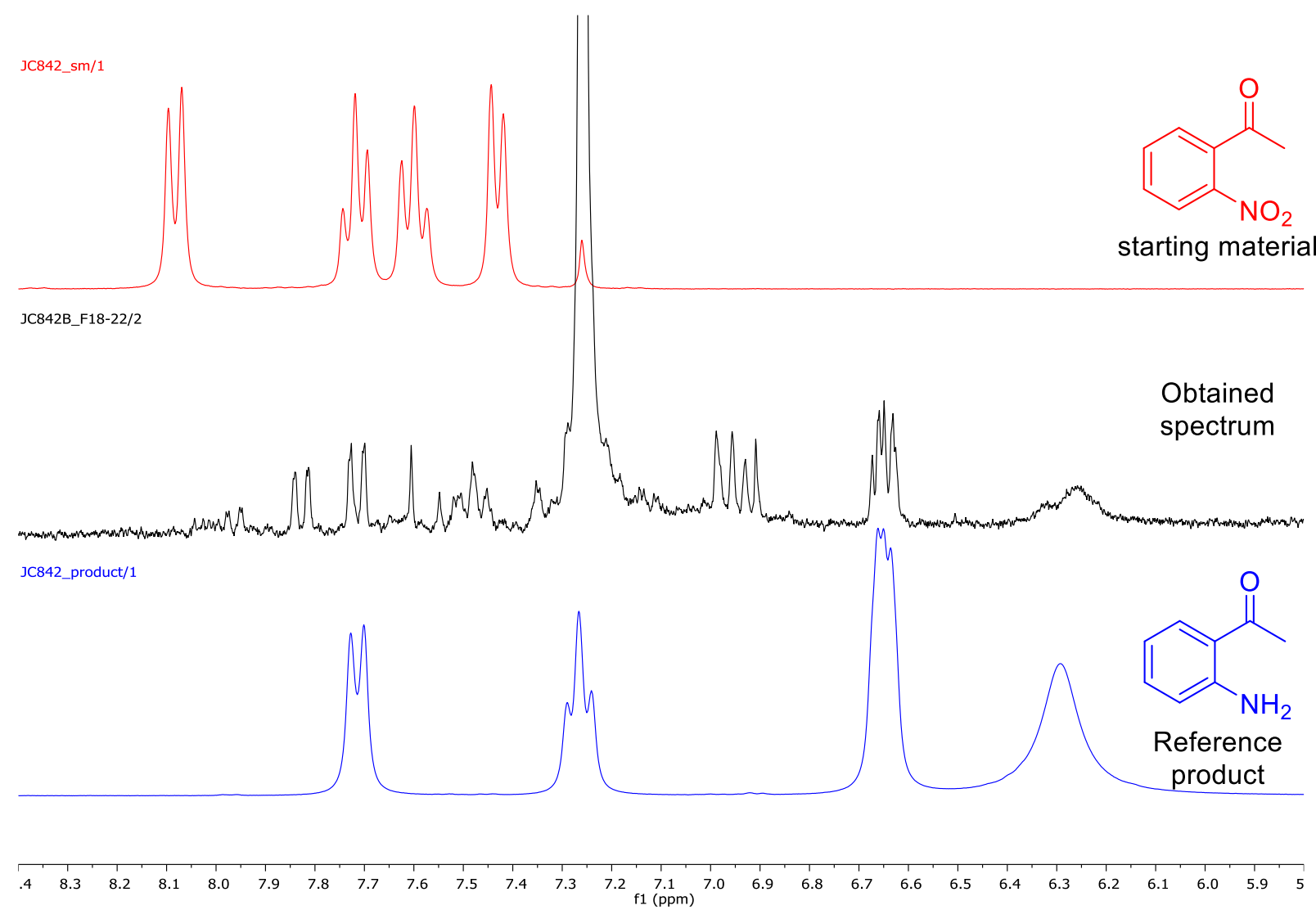

Figure $\mathrm{S}_{5}$. Spectral data of $\mathrm{H}_{\mathbf{2}}$ trapping by Pd-catalyzed hydrogenation of o-nitroacetonphenone

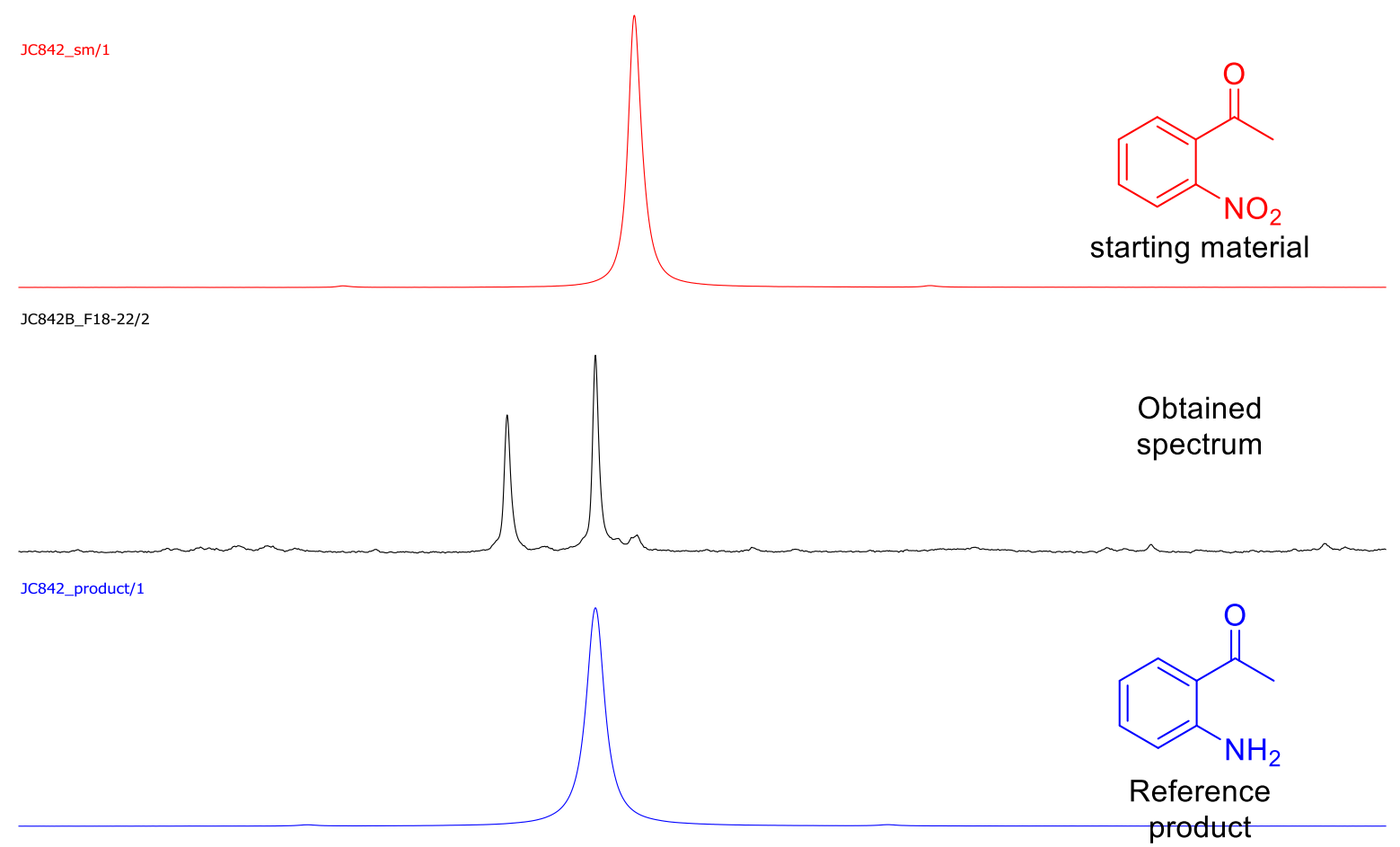

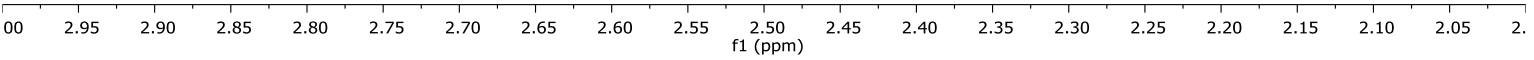

Figure S6. Spectral data of $\mathrm{H}_{2}$ trapping by Pd-catalyzed hydrogenation of o-nitroacetonphenone (continued) 


\section{Experimental information}

\subsection{Synthesis of starting materials}

General procedure B:

The $N$-uracil-amidines 1 have been synthesized according to our previously reported procedure. ${ }^{[4]}$

An oven-dried $10 \mathrm{~mL}$ pressure vial was loaded with $N, N^{\prime}$-dibenzyl-6-chlorouracil (1.0 equiv), the amidine (2.3 equiv) and anhydrous 3-ethyl-3-pentanol $(0.5 \mathrm{~mL})$, and closed with a septum. The resulting mixture was stirred at $100{ }^{\circ} \mathrm{C}$ under $\mathrm{N}_{2}$ atmosphere for $24 \mathrm{~h}$. The mixture was extracted with ethyl acetate $(60 \mathrm{~mL})$, and washed with water $(20 \mathrm{~mL})$. The organic layer was dried over $\mathrm{MgSO}_{4}$, filtered, concentrated under reduced pressure. The resulting residue was either purified by chromatography using the eluent indicated below.

\section{$N$-(1,3-dibenzyl-2,6-dioxo-1,2,3,6-tetrahydropyrimidin-4-yl)benzimidamide (1a) [JC721]}<smiles>N/C(=N\c1cc(=O)n(Cc2ccccc2)c(=O)n1Cc1ccccc1)c1ccccc1</smiles>
Synthesized according to general procedure $B$, using 1,3-dibenzyl-6chloropyridinine-2,4(1H,3H)-dione ( $6.54 \mathrm{~g}, 20.0 \mathrm{mmol}, 1.0$ equiv) benzamidine (5.53, $46 \mathrm{mmol}, 2.3$ equiv) and DBU (5.97 mL, $40 \mathrm{mmol}, 2.0$ equiv). The compound was purified by manual flash chromatography using cHex:EtOAc: $\mathrm{NEt}_{3}$ (gradient = 10:1:0.01-0:1:0.01). The title compound was isolated as a yellow solid $(5.12 \mathrm{~g}, 62 \%)$. Spectroscopic data are in accordance with the literature. ${ }^{[4]}$

$\mathbf{R}_{\mathbf{F}}=0.14$ (cHex : EtOAc: NEt $\left.; 1: 1: 0.01\right) .{ }^{1} \mathbf{H}$ NMR $\left(300 \mathrm{MHz}, \mathrm{CDCl}_{3}\right): \delta 7.76$ (d, J=7.1 Hz, 2H), $7.59-$ $7.51(\mathrm{~m}, 1 \mathrm{H}), 7.51-7.41(\mathrm{~m}, 4 \mathrm{H}), 7.35-7.19(\mathrm{~m}, 8 \mathrm{H}), 5.95(\mathrm{bs}, 2 \mathrm{H}), 5.28(\mathrm{~s}, 1 \mathrm{H}), 5.20(\mathrm{~s}, 2 \mathrm{H}), 5.12(\mathrm{~s}$, 2H) ppm. ${ }^{13} \mathrm{C}$ NMR (150.90 MHz, CDCl $)$ ): $\delta 163.5$ (C), 157.8 (C), 157.2 (C), 152.5 (C), 137.3 (C), 133.3 (C), $131.8(\mathrm{CH}), 128.6(\mathrm{CH}), 128.4(\mathrm{CH}), 128.4(\mathrm{CH}), 128.2(\mathrm{CH}), 127.6(\mathrm{CH}), 127.3(\mathrm{CH}), 127.3(\mathrm{CH}), 127.0$ (CH), 87.5 (CH), $46.6\left(\mathrm{CH}_{2}\right), 44.2\left(\mathrm{CH}_{2}\right)$ ppm (1 $\mathrm{CH}_{\text {Ar }}$ missing due to overlapping signal). HRMS (ESI): $\mathrm{m} / \mathrm{z}$ calculated for $\mathrm{C}_{25} \mathrm{H}_{23} \mathrm{~N}_{4} \mathrm{O}_{2}[\mathrm{M}+\mathrm{H}]^{+}=411.1816$, found $=411.1828$. M.p.: $168-170{ }^{\circ} \mathrm{C}$ (lit. $169{ }^{\circ} \mathrm{C}$ ) ${ }^{[4]}$.

$N$-(1,3-dibenzyl-2,6-dioxo-1,2,3,6-tetrahydropyrimidin-4-yl)-4-chlorobenzimidamide (1b) [HL110]<smiles>N/C(=N\c1cc(=O)n(Cc2ccccc2)c(=O)n1Cc1ccccc1)c1ccc(Cl)cc1</smiles>
Synthesized according to general procedure B, using 1,3-dibenzyl-6chloropyridinine-2,4(1H,3H)-dione ( $1.82 \mathrm{~g}, 5.6 \mathrm{mmol}, 1.0$ equiv), 4chlorobenzamidine hydrochloride $(1.60 \mathrm{~g}, 8.4 \mathrm{mmol}, 1.5$ equiv) and DBU (1.8 mL, $12.3 \mathrm{mmol}, 2.2$ equiv). The compound was purified by manual flash chromatography using cHex:EtOAc:NEt 3 (gradient $=$ 10:1:0.01-0:1:0.01). The title compound was isolated as a yellow solid $(0.925 \mathrm{~g}, 37 \%)$. Unknown compound according to a Sci-finder search.

$\mathbf{R}_{\mathrm{F}}=0.13$ (EtOAc: $c$ Hex : NEt $\left.\mathrm{N}_{3}=1: 1: 0.01\right) .{ }^{1} \mathbf{H}$ NMR $\left(300 \mathrm{MHz}, \mathrm{CDCl}_{3}\right): \delta 7.67(\mathrm{~d}, J=9.0 \mathrm{~Hz}, 2 \mathrm{H}), 7.44$ $(\mathrm{d}, J=9.0 \mathrm{~Hz}, 2 \mathrm{H}), 7.39(\mathrm{~d}, J=9.0 \mathrm{~Hz}, 2 \mathrm{H}), 7.33-7.26(\mathrm{~m}, 2 \mathrm{H}), 7.25-7.12(\mathrm{~m}, 6 \mathrm{H}), 5.93(\mathrm{~s}, 2 \mathrm{H}), 5.23(\mathrm{~s}$, 1H), 5.15 (s, 2H), 5.10 (s, 2H) ppm. $\left.{ }^{13} \mathrm{C} \mathrm{NMR} \mathrm{(125} \mathrm{MHz,} \mathrm{CDCl}\right): \delta 163.4$ (C), 156.8 (C), 152.5 (C), 138.3 (C), $137.24(\mathrm{C}), 137.22(\mathrm{C}), 131.6(\mathrm{C}), 129.0(\mathrm{CH}), 128.6(\mathrm{CH}), 128.5(\mathrm{CH}), 128.3(\mathrm{CH}), 127.6(\mathrm{CH}), 127.5$

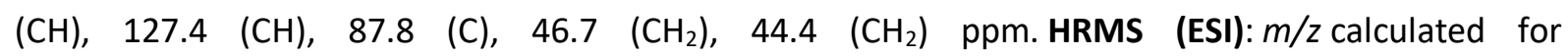
$\mathrm{C}_{25} \mathrm{H}_{22} \mathrm{~N}_{4} \mathrm{O}_{2} \mathrm{Cl}[\mathrm{M}+\mathrm{H}]^{+}=445.1431$, found $=445.1426$. M.p.: $115^{\circ} \mathrm{C}$.

$N$-(1,3-dibenzyl-2,6-dioxo-1,2,3,6-tetrahydropyrimidin-4-yl)-4-(trifluoromethyl)benzimidamide (1c)<smiles>N/C(=N\c1cc(=O)n(Cc2ccccc2)c(=O)n1Cc1ccccc1)c1ccc(C(F)(F)F)cc1</smiles>
[HL048]

Synthesized according to general procedure $B$, using 1,3-dibenzyl-6chloropyridinine-2,4(1H,3H)-dione ( $3.3 \mathrm{~g}, 10 \mathrm{mmol}, 1.0$ equiv), 4trifluoromethylbenzamidine hydrochloride dihydrate $(3.9 \mathrm{~g}, 15$ mmol, 1.5 equiv) and DBU (1.05 mL, $9 \mathrm{mmol})$. The compound was purified by manual flash chromatography using cHex:EtOAc:NEt ${ }_{3}$ 
(gradient $=10: 1: 0.01-0: 1: 0.01)$. The title compound was isolated as a light green solid (1.186 g, 25\%). Unknown compound according to a Sci-finder search.

$\mathbf{R}_{\mathbf{F}}=0.33$ (cHex : EtOAc : NEt3 = 1:1:0.01). ${ }^{1} \mathbf{H}$ NMR $\left(300 \mathrm{MHz}, \mathrm{CDCl}_{3}\right): \delta 7.81$ (d, J=6.0 Hz, 2H), 7.70 $(\mathrm{d}, J=6.0 \mathrm{~Hz}, 2 \mathrm{H}), 7.49(\mathrm{~d}, J=6.0 \mathrm{~Hz}, 2 \mathrm{H}), 7.35-7.27(\mathrm{~m}, 2 \mathrm{H}), 7.25-7.16(\mathrm{~m}, 6 \mathrm{H}), 5.71(\mathrm{~s}, 2 \mathrm{H}), 5.27(\mathrm{~s}$, $1 \mathrm{H}), 5.17(\mathrm{~s}, 2 \mathrm{H}), 5.15$ (s, 2H). ${ }^{13} \mathrm{C} \mathrm{NMR}\left(150.90 \mathrm{MHz}, \mathrm{CDCl}_{3}\right)$ : $\delta 163.4$ (C), 156.6 (C), 156.3 (C), 152.5 (C), 137.21 (C), $137.18(\mathrm{C}), 136.6(\mathrm{C}), 133.6(\mathrm{q}, 2 \mathrm{~J}=33 \mathrm{~Hz}, \mathrm{C}), 128.7(\mathrm{CH}), 128.5(\mathrm{CH}), 128.4(\mathrm{CH}), 127.8(\mathrm{CH})$, $127.53(\mathrm{CH}), 127.50(\mathrm{CH}), 127.47(\mathrm{CH}), 125.8(\mathrm{q}, 3 \mathrm{~J}=5 \mathrm{~Hz}), 125.7$ (q, 3J = $5 \mathrm{~Hz}, \mathrm{CH}), 123.5$ (q, $1 \mathrm{~J}=270$ $\mathrm{Hz}, \mathrm{CH}), 87.9(\mathrm{CH}), 46.8\left(\mathrm{CH}_{2}\right), 44.4\left(\mathrm{CH}_{2}\right)$. HRMS (ESI): $\mathrm{m} / z$ calculated for $\mathrm{C}_{26} \mathrm{H}_{22} \mathrm{~N}_{4} \mathrm{O}_{2} \mathrm{~F}_{3}[\mathrm{M}+\mathrm{H}]^{+}=$ 479.1666, found $=479.1689$. M.p.: $95^{\circ} \mathrm{C}$.

$N$-(1,3-dibenzyl-2,6-dioxo-1,2,3,6-tetrahydropyrimidin-4-yl)-4-methoxybenzimidamide (1d) [HL084]<smiles>COc1ccc(/C(N)=N/c2cc(=O)n(Cc3ccccc3)c(=O)n2Cc2ccccc2)cc1</smiles>
Synthesized according to general procedure $\mathrm{B}$, using 1,3-dibenzyl-6chloropyridinine-2,4(1H,3H)-dione (1.961 g, $6 \mathrm{mmol}, 1.0$ equiv), 4methoxybenzamidine hydrochloride $(1.68 \mathrm{~g}, 9 \mathrm{mmol}, 1.5$ equiv) and DBU $(2.0 \mathrm{~mL}, 13.2 \mathrm{mmol})$. The compound was purified by manual flash chromatography using cHex:EtOAc:NEt ${ }_{3}$ (gradient $=10: 1: 0.01-$ $0: 1: 0.01)$. The title compound was isolated as a white solid (1.13 g, 43\%). Unknown compound according to a Sci-finder search.

$\mathbf{R}_{\mathbf{F}}=0.17$ (cHex : EtOAc: NEt $\left.{ }_{3}=1: 1: 0.01\right) .{ }^{1} \mathbf{H}$ NMR $\left(300 \mathrm{MHz}, \mathrm{CDCl}_{3}\right): \delta 7.68(\mathrm{~d}, J=8.3 \mathrm{~Hz}, 2 \mathrm{H}), 7.49$ $(\mathrm{d}, J=6.4 \mathrm{~Hz}, 2 \mathrm{H}), 7.38-7.13(\mathrm{~m}, 8 \mathrm{H}), 6.93(\mathrm{~d}, J=8.4 \mathrm{~Hz}, 2 \mathrm{H}), 5.54(\mathrm{bs}, 2 \mathrm{H}), 5.26(\mathrm{~s}, 1 \mathrm{H}), 5.19(\mathrm{~s}, 2 \mathrm{H})$, $5.16(\mathrm{~s}, 2 \mathrm{H}), 3.86(\mathrm{~s}, 3 \mathrm{H}) \mathrm{ppm} .{ }^{13} \mathrm{C}$ NMR $\left(151 \mathrm{MHz}, \mathrm{CDCl}_{3}\right): \delta 163.3$ (C), 162.7 (C), 157.0 (C), 156.9 (C), $152.6(\mathrm{C}), 137.5(\mathrm{C}), 137.4(\mathrm{C}), 128.85(\mathrm{CH}), 128.83(\mathrm{CH}), 128.4(\mathrm{CH}), 128.3(\mathrm{CH}), 127.8(\mathrm{CH}), 127.4(\mathrm{CH})$, $127.3(\mathrm{CH}), 125.3(\mathrm{C}), 114.0(\mathrm{CH}), 87.7(\mathrm{CH}), 55.5\left(\mathrm{CH}_{3}\right), 46.6\left(\mathrm{CH}_{2}\right), 43.3\left(\mathrm{CH}_{2}\right)$ ppm. HRMS (ESI): $m / z$ calculated for $\mathrm{C}_{26} \mathrm{H}_{25} \mathrm{~N}_{4} \mathrm{O}_{3}[\mathrm{M}+\mathrm{H}]^{+}=441.1923$, found $=441.1921$. M.p.: $197^{\circ} \mathrm{C}$.

\section{$N$-(1,3-dibenzyl-2,6-dioxo-1,2,3,6-tetrahydropyrimidin-4-yl)acetimidamide (1e) [HL080]}<smiles>C/C(N)=N/c1cc(=O)n(Cc2ccccc2)c(=O)n1Cc1ccccc1</smiles>
Synthesized according to general procedure B, using 1,3-dibenzyl-6chloropyridinine-2,4(1H,3H)-dione $(3.27 \mathrm{~g}, 10 \mathrm{mmol}, 1.0$ equiv), ethanimidamide hydrochloride ( $1.48 \mathrm{~g}, 15 \mathrm{mmol}, 1.5$ equiv) and DBU ( $3.3 \mathrm{~mL}, 22$ mmol, 2.2 equiv). The compound was purified by manual flash chromatography using cHex:EtOAc:NEt ${ }_{3}$ (gradient = 10:1:0.01-1:1:0.01). The title compound was isolated as a white solid ( $2.50 \mathrm{~g}, 73 \%)$. Unknown compound according to a Sci-

finder search.

$\mathbf{R}_{\mathrm{F}}=0.28$ (cHex : EtOAc: $\left.\mathrm{NEt}_{3}=1: 1: 0.1\right) .{ }^{1} \mathbf{H}$ NMR $\left(600 \mathrm{MHz}, \mathrm{CDCl}_{3}\right): \delta 7.49(\mathrm{~d}, J=6.0 \mathrm{~Hz}, 2 \mathrm{H}), 7.35-7.26$ $(\mathrm{m}, 4 \mathrm{H}), 7.25-7.19(\mathrm{~m}, 4 \mathrm{H}), 5.29$ (bs, 1H), $5.15(\mathrm{~s}, 2 \mathrm{H}), 5.08(\mathrm{~s}, 3 \mathrm{H}), 4.96$ (bs, $1 \mathrm{H}), 1.78(\mathrm{~s}, 3 \mathrm{H}) .{ }^{13} \mathrm{C}$ NMR (125 MHz, CDCl $): \delta{ }^{13} \mathrm{C}$ NMR (151 MHz, DMSO-d6) $\delta 162.0$ (C), 161.5 (C), 157.6, (C) 152.1 (C), $137.9(\mathrm{C}), 137.9(\mathrm{C}), 128.3(\mathrm{CH}), 128.2(\mathrm{CH}), 127.4(\mathrm{CH}), 126.94(\mathrm{CH}), 126.92(\mathrm{CH}), 126.89(\mathrm{CH}), 86.7$ (CH), $45.5(\mathrm{C}), 43.3(\mathrm{C}), 18.9\left(\mathrm{CH}_{3}\right)$, HRMS (ESI): $\mathrm{m} / z$ calculated for $\mathrm{C}_{20} \mathrm{H}_{21} \mathrm{~N}_{4} \mathrm{O}_{2}[\mathrm{M}+\mathrm{H}]^{+}=349.1657$, found $=349.1659$. M.p.: $163^{\circ} \mathrm{C}$.

\section{$N$-(1,3-dibenzyl-2,6-dioxo-1,2,3,6-tetrahydropyrimidin-4-yl)isobutyrimidamide (1f) [HL095]}<smiles>CC(C)C(N)=Nc1cc(=O)n(Cc2ccccc2)c(=O)n1Cc1ccccc1</smiles>
Synthesized according to general procedure $B$, using 1,3-dibenzyl-6chloropyridinine-2,4(1H,3H)-dione (3.45 g, $10.6 \mathrm{mmol}, 1.0$ equiv) 2methylpropanimidamide hydrochloride $(1.94 \mathrm{~g}, 15.8 \mathrm{mmol}, 1.5$ equiv) and DBU ( $3.5 \mathrm{~mL}, 23.2 \mathrm{mmol}, 2.2$ equiv). The compound was purified by manual flash chromatography using cHex:EtOAc:NEt ${ }_{3}$ (gradient = 10:1:0.01-0:1:0.01). The title compound was isolated as an off-white solid ( $2.17 \mathrm{~g}, 55 \%)$. Unknown compound according to a Sci-finder search.

$\mathbf{R}_{\mathbf{F}}=0.17$ (cHex : EtOAc : NEt $\left.{ }_{3}=1: 1: 0.01\right) .{ }^{1} \mathbf{H} \mathbf{~ N M R}\left(300 \mathrm{MHz}, \mathrm{CDCl}_{3}\right): \delta 7.44(\mathrm{dd}, J=6.0,9.0 \mathrm{~Hz}, 2 \mathrm{H})$, 7.35 - $7.26(\mathrm{~m}, 2 \mathrm{H}), 7.25-7.15(\mathrm{~m}, 6 \mathrm{H}), 5.35$ (bs, 2H), $5.16(\mathrm{~s}, 1 \mathrm{H}), 5.11(\mathrm{~s}, 2 \mathrm{H}), 5.10(\mathrm{~s}, 2 \mathrm{H}), 2.42(\mathrm{sep}, J=$ $6.0 \mathrm{~Hz}, 1 \mathrm{H}$ ), 1.09 (d, $J=6.0 \mathrm{~Hz}, 6 \mathrm{H})$ ppm. ${ }^{13} \mathrm{C} \mathrm{NMR}\left(125 \mathrm{MHz}, \mathrm{CDCl}_{3}\right): \delta 167.0$ (C), 163.4 (C), 157.2 (C), $152.5(\mathrm{C}), 137.3(\mathrm{C}), 137.3(\mathrm{C}), 128.4(\mathrm{CH}), 128.4(\mathrm{CH}), 128.2(\mathrm{CH}), 127.2(\mathrm{CH}), 127.2(\mathrm{CH}), 127.1(\mathrm{CH})$, 
$87.3(\mathrm{CH}), \quad 46.3\left(\mathrm{CH}_{2}\right), \quad 44.2\left(\mathrm{CH}_{2}\right), \quad 34.7(\mathrm{CH}), \quad 20.1 \quad\left(\mathrm{CH}_{3}\right)$. HRMS (ESI): $m / z$ calculated for $\mathrm{C}_{22} \mathrm{H}_{25} \mathrm{~N}_{4} \mathrm{O}_{2}[\mathrm{M}+\mathrm{H}]^{+}=377.1972$, found $=377.1972$. M.p.: $152^{\circ} \mathrm{C}$.

\section{$N^{\prime}$-(1,3-dibenzyl-2,6-dioxo-1,2,3,6-tetrahydropyrimidin-4-yl)pivalimidamide (1g) [HL117]}<smiles>CC(C)(C)/C(N)=N/c1cc(=O)n(Cc2ccccc2)c(=O)n1Cc1ccccc1</smiles>
Synthesized according to general procedure $B$, using 1,3-dibenzyl-6chloropyridinine-2,4(1H,3H)-dione (1.14 g, $3.5 \mathrm{mmol}, 1.0$ equiv) 2,2dimethylpropanimidamide hydrochloride ( $710 \mathrm{mg}, 5.2 \mathrm{mmol}, 1.5$ equiv) and DBU ( $1.1 \mathrm{~mL}, 9.6 \mathrm{mmol}, 2.2$ equiv). The compound was purified by manual flash chromatography using cHex:EtOAc: $\mathrm{NEt}_{3}$ (gradient = 10:1:0.01-0:1:0.01). The title compound was isolated as a white solid (0.855 g, 62\%). Unknown compound according to a Sci-finder search.

$\mathbf{R}_{\mathrm{F}}=0.40$ (cHex : EtOAc: $\left.\mathrm{NEt}_{3}=1: 1: 0.01\right) .{ }^{1} \mathrm{H}$ NMR $\left(300 \mathrm{MHz}, \mathrm{CDCl}_{3}\right): \delta 7.45$ (d, $\left.J=10.0 \mathrm{~Hz}, 2 \mathrm{H}\right), 7.32-$ $7.26(\mathrm{~m}, 3 \mathrm{H}), 7.25-7.17(\mathrm{~m}, 5 \mathrm{H}), 5.34$ (bs, 2H), $5.17(\mathrm{~s}, 1 \mathrm{H}), 5.12(\mathrm{~s}, 2 \mathrm{H}), 5.11(\mathrm{~s}, 2 \mathrm{H}), 1.16(\mathrm{~s}, 9 \mathrm{H})$ ppm. ${ }^{13} \mathrm{C} \mathrm{NMR}\left(125 \mathrm{MHz}, \mathrm{CDCl}_{3}\right): \delta 168.5$ (C), 163.4 (C), 157.2 (C), 152.6 (C), 137.4 (C), 137.3 (C), 128.7 $(\mathrm{CH}), 128.4(\mathrm{CH}), 128.3(\mathrm{CH}), 127.3(\mathrm{CH}), 127.2(\mathrm{CH}), 127.0(\mathrm{CH}), 87.1(\mathrm{CH}), 46.3\left(\mathrm{CH}_{2}\right), 44.2\left(\mathrm{CH}_{2}\right), 37.7$ (C), $28.0\left(\mathrm{CH}_{3}\right)$ ppm. HRMS (ESI): $m / z$ calculated for $\mathrm{C}_{23} \mathrm{H}_{27} \mathrm{~N}_{4} \mathrm{O}_{2}[\mathrm{M}+\mathrm{H}]^{+}=391.2132$, found $=$ 391.2129. M.p.: $159^{\circ} \mathrm{C}$.

\subsection{Synthesis of pyrimidouracils}

\section{General procedure C:}

A $10 \mathrm{~mL}$ pressure vial, equipped with a stir bar, was charged with $\mathrm{N}$-(1,3-dibenzyluracil)benzimidamide 1 ( $0.5 \mathrm{mmol}, 1.0$ equiv), $\mathrm{Ni}(\mathrm{OAc})_{2} .4 \mathrm{H}_{2} \mathrm{O}(6 \mathrm{mg}, 0.025 \mathrm{mmol}, 5 \mathrm{~mol} \%)$ and anisole $(2.0 \mathrm{~mL})$. Hereafter, the isocyanide ( $1.0 \mathrm{mmol}, 2.0$ equiv or $1.5 \mathrm{mmol}, 3.0$ eq.) was added dropwise with a syringe (if liquid) or with a funnel (if solid). Subsequently, the reaction mixture was again heated to $50^{\circ} \mathrm{C}$ in an oil bath for 18 hours. The reaction mixture was kept under air atmosphere with a balloon (connection with a needle through the septum), although no significantly different results were obtained if the vessel was left open to air. When the reaction mixture was cooled down it was diluted with ethyl acetate $(60 \mathrm{~mL})$, and washed with $\mathrm{NH}_{4} \mathrm{OH}$ (aq.)/saturated brine $(3: 2,20 \mathrm{~mL}$ ). The organic fraction was dried over anhydrous $\mathrm{Na}_{2} \mathrm{SO}_{4}$, filtered, concentrated in vacuo, and loaded onto silica. The products were purified by flash column chromatography on $\mathrm{SiO}_{2}$, using the eluent indicated below.

\section{1,3-dibenzyl-5-(tert-butylamino)-7-phenylpyrimido[4,5-d]pyrimidine-2,4(1H,3H)-dione (3a)}<smiles>CC(C)(C)Nc1nc(-c2ccccc2)nc2c1c(=O)n(Cc1ccccc1)c(=O)n2Cc1ccccc1</smiles>

To a solution of $\mathrm{N}$-(1,3-dibenzyluracil)benzimidamide $(\mathbf{1 a}, 410 \mathrm{mg}, 1.0$ mmol, 1.0 equiv) and $\mathrm{Ni}(\mathrm{OAc})_{2} .4 \mathrm{H}_{2} \mathrm{O}(0.011 \mathrm{~g}, 0.05 \mathrm{mmol}, 0.05$ equiv) in anisole $(4.0 \mathrm{~mL})$ was added tert-butyl isocyanide $(2 \mathrm{a}, 226 \mu \mathrm{L}, 2.0 \mathrm{mmol}, 2.0$ equiv). The mixture was stirred open to air for 16 hours, after which the reaction was cooled, and the mixture was diluted with $50 \mathrm{~mL}$ EtOAc. The crude product was washed subsequently with $\mathrm{NH}_{4} \mathrm{OH}_{\text {(aq.), }}$, sat. $\mathrm{NaHCO}_{3}$, and brine. The organic fraction was dried over anhydrous $\mathrm{NaSO}_{4}$, concentrated in vacuo, and the crude product was loaded onto $\mathrm{SiO}_{2}$ with $\mathrm{DCM}$. The compound was purified by an automated flash chromatography system using cHex:EtOAc (gradient = 1:0-3:1). The title compound was isolated as a white solid (476 mg, 97\%). Unknown compound according to a Sci-finder search. $\mathbf{R}_{\mathrm{F}}=0.16$ (cHex : EtOAc : NEt $\left.; 9: 1: 0.01\right) .{ }^{1} \mathbf{H} \mathbf{~ N M R}\left(300 \mathrm{MHz}, \mathrm{CDCl}_{3}\right): \delta 9.22$ (bs, $\left.1 \mathrm{H}\right), 8.47$ (d, $J=8.0$ $\mathrm{Hz}, 1 \mathrm{H}), 7.61-7.42(\mathrm{~m}, 7 \mathrm{H}), 7.39-7.20(\mathrm{~m}, 7 \mathrm{H}), 5.59(\mathrm{~s}, 2 \mathrm{H}), 5.21(\mathrm{~s}, 2 \mathrm{H}), 1.62(\mathrm{~s}, 9 \mathrm{H}) \mathrm{ppm} .{ }^{13} \mathrm{C}$ NMR $\left(100 \mathrm{MHz}_{\mathrm{CDCl}}\right)$ ) $\delta: 165.6$ (C), 162.9 (C), 161.5 (C), 157.5 (C), 151.4 (C), 137.7 (C), 137.4 (C), 136.9 (C), $131.7(\mathrm{CH}), 129.1(\mathrm{CH}), 128.76(\mathrm{CH}), 128.75(\mathrm{CH}), 128.62(\mathrm{CH}), 128.61(\mathrm{CH}), 128.55,127.76(\mathrm{CH})$, $127.74(\mathrm{CH}), 89.0(\mathrm{C}), 52.8(\mathrm{C}), 45.6\left(\mathrm{CH}_{2}\right), 44.6\left(\mathrm{CH}_{2}\right), 29.3\left(\mathrm{CH}_{3}\right) \mathrm{ppm}$. HRMS (ESI): $\mathrm{m} / \mathrm{z}$ calculated for $\mathrm{C}_{30} \mathrm{H}_{30} \mathrm{~N}_{5} \mathrm{O}_{2}[\mathrm{M}+\mathrm{H}]^{+}=492.2394$, found $=492.2390$. M.p.: $189^{\circ} \mathrm{C}$. 


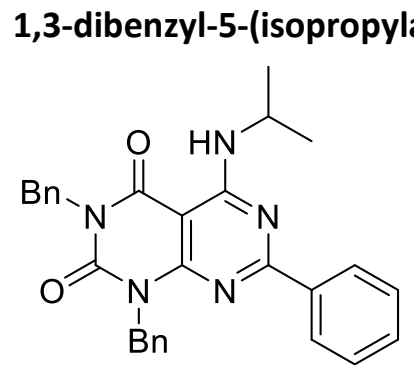
mino)-7-phenylpyrimido[4,5- $d]$ pyrimidine-2,4(1H,3H)-dione (3b) [BM1180] The general procedure $\mathrm{C}$ was applied using $\mathrm{N}-(1,3-$ dibenzyluracil)benzimidamide (1a, $205 \mathrm{mg}, 0.5 \mathrm{mmol}, 1.0$ equiv) and isopropyl isocyanide ( $140 \mu \mathrm{L}, 1.5 \mathrm{mmol}, 3.0$ equiv). The compound was purified by an automated flash chromatography system using a heptane / EtOAc gradient (from 100\% heptane to $35 \%$ ethyl acetate over 20 min, 35 $\mathrm{mL} / \mathrm{min}$ ). The title compound was isolated as a white solid (141 $\mathrm{mg}, 59 \%)$. Unknown compound according to a Sci-finder search.

$\mathbf{R}_{\mathbf{F}}=0.12$ (cHex : EtOAc: $\left.\mathrm{NEt}_{3} ; 9: 1: 0.01\right) .{ }^{1} \mathbf{H} \mathbf{~ N M R}\left(400 \mathrm{MHz}, \mathrm{CDCl}_{3}\right) \delta: 9.04(\mathrm{bd}, J=7.1 \mathrm{~Hz}, 1 \mathrm{H}), 8.47$ $(\mathrm{d}, J=6.8 \mathrm{~Hz}, 2 \mathrm{H}), 7.55-7.46(\mathrm{~m}, 7 \mathrm{H}), 7.32-7.24(\mathrm{~m}, 6 \mathrm{H}), 5.59(\mathrm{~s}, 2 \mathrm{H}), 5.21(\mathrm{~s}, 2 \mathrm{H}), 4.62$ (oct, $J=6.6$ $\mathrm{Hz}, 1 \mathrm{H}), 1.35(\mathrm{~d}, J=6.6 \mathrm{~Hz}, 6 \mathrm{H}) \mathrm{ppm} .{ }^{13} \mathrm{C}$ NMR $\left(100 \mathrm{MHz}, \mathrm{CDCl}_{3}\right) \delta: 166.0$ (C), 162.8 (C), 161.1 (C), $157.5(\mathrm{C}), 151.5(\mathrm{C}), 137.5(\mathrm{C}), 136.9(\mathrm{C}), 131.7(\mathrm{C}), 129.1(\mathrm{CH}), 128.8(\mathrm{CH}), 128.7(\mathrm{CH}), 128.6(\mathrm{CH})$, 128.6 (CH), $128.5(\mathrm{CH}), 127.8(\mathrm{CH}), 127.8(\mathrm{CH}), 88.6(\mathrm{C}), 45.7\left(\mathrm{CH}_{2}\right), 44.6\left(\mathrm{CH}_{2}\right), 43.0(\mathrm{CH}), 22.8\left(\mathrm{CH}_{3}\right)$ ppm ( $1 \mathrm{CH}_{\text {Ar }}$ missing due to overlapping signals). HRMS (ESI): $m / z$ calculated for $\mathrm{C}_{29} \mathrm{H}_{28} \mathrm{~N}_{5} \mathrm{O}_{2}[\mathrm{M}+\mathrm{H}]^{+}=$ 478.2243, found $=478.2258$. M.p.: $183^{\circ} \mathrm{C}$.

1,3-dibenzyl-5-(cyclohexylamino)-7-phenylpyrimido[4,5-d]pyrimidine-2,4(1H,3H)-dione (3c) [BM1182]<smiles>O=c1c2c(NC3CCCCC3)nc(-c3ccccc3)nc2n(Cc2ccccc2)c(=O)n1Cc1ccccc1</smiles>

The general procedure $\mathrm{C}$ was applied using $\mathrm{N}-(1,3-$ dibenzyluracil)benzimidamide (1a, $205 \mathrm{mg}, 0.5 \mathrm{mmol}, 1.0$ equiv) and cyclohexyl isocyanide (194 $\mu \mathrm{L}, 1.5 \mathrm{mmol}, 3.0$ equiv). The compound was purified by an automated flash chromatography system using a heptane / EtOAc gradient (from 100\% heptane to $20 \%$ ethyl acetate over $20 \mathrm{~min}, 35$ $\mathrm{mL} / \mathrm{min}$ ). The title compound was isolated as a white solid (206 mg, 80\%). Unknown compound according to a Sci-finder search.

$\mathbf{R}_{\mathbf{F}}=0.12$ (cHex : EtOAc: $\left.\mathrm{NEt}_{3} ; 9: 1: 0.01\right) .{ }^{1} \mathbf{H}$ NMR $\left(400 \mathrm{MHz}, \mathrm{CDCl}_{3}\right) \delta: 8.99$ (bd, $J=7.5 \mathrm{~Hz}, 1 \mathrm{H}), 8.47-8.43(\mathrm{~m}, 2 \mathrm{H}), 7.52(\mathrm{~d}, J=7.0 \mathrm{~Hz}, 2 \mathrm{H}), 7.51-7.45(\mathrm{~m}, 5 \mathrm{H}), 7.32-7.20(\mathrm{~m}, 6 \mathrm{H})$, $5.55(\mathrm{~s}, 2 \mathrm{H}), 5.19(\mathrm{~s}, 2 \mathrm{H}), 4.36-4.27(\mathrm{~m}, 1 \mathrm{H}), 2.11-2.04(\mathrm{~m}, 2 \mathrm{H}), 1.86-1.77(\mathrm{~m}, 2 \mathrm{H}), 1.71-1.63(\mathrm{~m}$, 1H), 1.53 - 1.25 (m, 5H). ${ }^{13} \mathrm{C}$ NMR (100 MHz, CDCl $)$ $\delta: 165.9$ (C), 162.7 (C), 161.0 (C), 157.5 (C), 151.4 (C), $137.5(\mathrm{C}), 137.3(\mathrm{C}), 136.9(\mathrm{C}), 131.6(\mathrm{CH}), 129.0(\mathrm{CH}), 128.8(\mathrm{CH}), 128.6(\mathrm{CH}), 128.6(\mathrm{CH}), 128.4$ $(\mathrm{CH}), 127.7(\mathrm{CH}), 127.7(\mathrm{CH}), 88.5(\mathrm{C}), 49.8(\mathrm{CH}), 45.6\left(\mathrm{CH}_{2}\right), 44.5\left(\mathrm{CH}_{2}\right), 32.8\left(\mathrm{CH}_{2}\right), 25.8\left(\mathrm{CH}_{2}\right), 24.9\left(\mathrm{CH}_{2}\right)$ ppm, $\left(1 \mathrm{CH}_{\mathrm{Ar}}\right.$ missing due to overlapping signals). $\mathrm{HRMS}$ (ESI): $\mathrm{m} / z$ calculated for $\mathrm{C}_{32} \mathrm{H}_{32} \mathrm{~N}_{5} \mathrm{O}_{2}[\mathrm{M}+\mathrm{H}]^{+}=$ 519.2556, found $=519.2570$. M.p.: $205^{\circ} \mathrm{C}$.

\section{1,3-dibenzyl-5-(pentylamino)-7-phenylpyrimido[4,5-d]pyrimidine-2,4(1H,3H)-dione (3d)} [BM1165]<smiles>CCCCCNc1nc(-c2ccccc2)nc2c1c(=O)n(Cc1ccccc1)c(=O)n2Cc1ccccc1</smiles>

The general procedure $\mathrm{C}$ was applied using $\mathrm{N}-(1,3-$ dibenzyluracil)benzimidamide (1a, $205 \mathrm{mg}, 0.5 \mathrm{mmol}, 1.0$ equiv) and $n$ pentyl isocyanide ( $130 \mu \mathrm{L}, 1.5 \mathrm{mmol}, 3.0$ equiv). The compound was purified by an automated flash chromatography system using a heptane / EtOAc gradient (from $100 \%$ heptane to $10 \%$ ethyl acetate over 30 min, $35 \mathrm{~mL} / \mathrm{min}$ ). The title compound was isolated as a white solid (205 mg, 82\%). Unknown compound according to a Sci-finder search.

$\mathbf{R}_{\mathrm{F}}=0.12$ (cHex : EtOAc: NEt $\left.{ }_{3} ; 9: 1: 0.01\right) .{ }^{1} \mathbf{H}$ NMR $\left(400 \mathrm{MHz}, \mathrm{CDCl}_{3}\right) \delta: 9.04(\mathrm{bt}, J=5.1 \mathrm{~Hz}, 1 \mathrm{H}), 8.48$ $(\mathrm{d}, J=6.8 \mathrm{~Hz}, 2 \mathrm{H}), 7.56-7.46(\mathrm{~m}, 7 \mathrm{H}), 7.34-7.23(\mathrm{~m}, 6 \mathrm{H}), 5.59(\mathrm{~s}, 2 \mathrm{H}), 5.21(\mathrm{~s}, 2 \mathrm{H}), 3.70(\mathrm{q}, J=6.8 \mathrm{~Hz}$, $2 \mathrm{H}), 1.71$ (quint, $J=7.1 \mathrm{~Hz}, 2 \mathrm{H}), 1.45-1.37(\mathrm{~m}, 4 \mathrm{H}), 0.93(\mathrm{t}, J=6.9 \mathrm{~Hz}, 3 \mathrm{H}) \mathrm{ppm} .{ }^{13} \mathrm{C} \mathrm{NMR}(100 \mathrm{MHz}$, $\left.\mathrm{CDCl}_{3}\right) \delta: 166.1$ (C), $162.8(\mathrm{C}), 161.9$ (C), $157.5(\mathrm{C}), 151.5(\mathrm{C}), 137.5$ (C), 137.3 (C), 136.9 (C), $131.7(\mathrm{CH})$, $129.1(\mathrm{CH}), 128.8(\mathrm{CH}), 128.8(\mathrm{CH}), 128.7(\mathrm{CH}), 128.6(\mathrm{CH}), 128.5(\mathrm{CH}), 127.8(\mathrm{CH}), 127.8(\mathrm{CH}), 88.8(\mathrm{C})$, $45.7\left(\mathrm{CH}_{2}\right), 44.6\left(\mathrm{CH}_{2}\right), 41.1\left(\mathrm{CH}_{2}\right), 29.4\left(\mathrm{CH}_{2}\right), 29.3\left(\mathrm{CH}_{2}\right), 22.6\left(\mathrm{CH}_{2}\right), 14.1\left(\mathrm{CH}_{3}\right)$ ppm. HRMS (ESI): $m / z$ calculated for $\mathrm{C}_{31} \mathrm{H}_{32} \mathrm{~N}_{5} \mathrm{O}_{2}[\mathrm{M}+\mathrm{H}]^{+}=506.2556$, found $=506.2559$. M.p.: $144{ }^{\circ} \mathrm{C}$. 


\section{1,3-dibenzyl-5-(benzylamino)-7-phenylpyrimido[4,5-d]pyrimidine-2,4-(1H,3H)-dione (3e)}

[BM1183]<smiles>O=c1c2c(NCc3ccccc3)nc(-c3ccccc3)nc2n(Cc2ccccc2)c(=O)n1Cc1ccccc1</smiles>

The general procedure $C$ was applied using $\mathrm{N}-(1,3-$ dibenzyluracil)benzimidamide (1a, $205 \mathrm{mg}, 0.5 \mathrm{mmol}, 1.0$ equiv) and benzyl isocyanide (122 $\mu \mathrm{L}, 1.5 \mathrm{mmol}, 3.0$ equiv). The compound was purified by an automated flash chromatography system using a heptane / EtOAc gradient (from 100\% heptane to $16 \%$ ethyl acetate over 20 min, 35 $\mathrm{mL} / \mathrm{min}$ ). The title compound was isolated as yellowish solid (204 mg, 78\%). Unknown compound according to a Sci-finder search.

$\mathbf{R}_{\mathrm{F}}=0.07$ (cHex : EtOAc: NEt $\left.{ }_{3} ; 9: 1: 0.01\right) .{ }^{1} \mathbf{H}$ NMR $\left(400 \mathrm{MHz}, \mathrm{CDCl}_{3}\right) \delta: 9.38$ (bt, J=5.4 Hz, 1H), 8.46 $(\mathrm{d}, J=7.0 \mathrm{~Hz}, 2 \mathrm{H}), 7.55-7.24(\mathrm{~m}, 18 \mathrm{H}), 5.58(\mathrm{~s}, 2 \mathrm{H}), 5.19(\mathrm{~s}, 2 \mathrm{H}), 4.92(\mathrm{~d}, J=5.9 \mathrm{~Hz}, 2 \mathrm{H}) \mathrm{ppm} .{ }^{13} \mathrm{C}$ NMR $\left(100 \mathrm{MHz}, \mathrm{CDCl}_{3}\right)$ \&: 166.2 (C), 162.8 (C), 161.9 (C), 157.6 (C), 151.4 (C), 138.5 (C), 137.3 (C), 137.3 (C), $136.8(\mathrm{C}), 131.9(\mathrm{CH}), 129.2(\mathrm{CH}), 128.9(\mathrm{CH}), 128.9(\mathrm{CH}), 128.8(\mathrm{CH}), 128.6(\mathrm{CH}), 128.5(\mathrm{CH}), 127.9(\mathrm{CH})$, $127.8(\mathrm{CH}), 127.8(\mathrm{CH}), 127.6(\mathrm{CH}), 88.9(\mathrm{C}), 45.7\left(\mathrm{CH}_{2}\right), 44.9\left(\mathrm{CH}_{2}\right), 44.7\left(\mathrm{CH}_{2}\right)$ ppm $\left(1 \mathrm{CH}_{\text {Ar }}\right.$ missing due to overlapping signals). HRMS (ESI): $\mathrm{m} / z$ calculated for $\mathrm{C}_{33} \mathrm{H}_{28} \mathrm{~N}_{5} \mathrm{O}_{2}[\mathrm{M}+\mathrm{H}]^{+}=526.2243$, found $=$ 526.2263. M.p.: $192^{\circ} \mathrm{C}$.

1,3-dibenzyl-7-phenyl-5-[(2,4,4-trimethylpentan-2-yl)amino]pyrimido[4,5-d]pyrimidine-2,4(1H,3H)dione (3f) [BM1173]<smiles>CC(C)(C)CC(C)(C)Nc1nc(-c2ccccc2)nc2c1c(=O)n(Cc1ccccc1)c(=O)n2Cc1ccccc1</smiles>

The general procedure $C$ was applied using $\mathrm{N}-(1,3-$ dibenzyluracil)benzimidamide (1a, $205 \mathrm{mg}, 0.5 \mathrm{mmol}, 1.0$ equiv) and 1,1,3,3-tetramethylbutyl isocyanide ( $175 \mu \mathrm{L}, 1.5 \mathrm{mmol}, 3.0$ equiv). The compound was purified by an automated flash chromatography system using a heptane / EtOAc gradient (from 100\% heptane to $16 \%$ ethyl acetate over $20 \mathrm{~min}, 10 \mathrm{~mL} / \mathrm{min}$ ). The title compound was isolated as yellowish solid (235 mg, 86\%). Unknown compound according to a Sci-finder search. $\mathbf{R}_{\mathbf{F}}=0.16$ (cHex : EtOAc: $\left.\mathrm{NEt}_{3} ; 9: 1: 0.01\right) .{ }^{1} \mathbf{H} \mathbf{~ N M R}\left(400 \mathrm{MHz}, \mathrm{CDCl}_{3}\right) \delta: 9.28$ (bs, $1 \mathrm{H}), 8.22(\mathrm{~d}, J=6.3 \mathrm{~Hz}, 2 \mathrm{H}), 7.54(\mathrm{~d}, J=7.0 \mathrm{~Hz}, 2 \mathrm{H}), 7.52-7.45(\mathrm{~m}, 5 \mathrm{H}), 7.33-7.22(\mathrm{~m}, 6 \mathrm{H}), 5.57(\mathrm{~s}$, 2H), $5.20(\mathrm{~s}, 2 \mathrm{H}), 2.09(\mathrm{~s}, 2 \mathrm{H}), 1.65(\mathrm{~s}, 6 \mathrm{H}), 1.0(\mathrm{~s}, 9 \mathrm{H}) \mathrm{ppm} .{ }^{13} \mathrm{C} \mathrm{NMR}\left(100 \mathrm{MHz}, \mathrm{CDCl}_{3}\right) \delta: 165.2(\mathrm{C}), 162.9$ (C), 161.4 (C), 157.5 (C), 151.3 (C), 137.7 (C), 137.4 (C), 136.9 (C), $131.6(\mathrm{CH}), 129.1(\mathrm{CH}), 128.9(\mathrm{CH})$, $128.9(\mathrm{CH}), 128.6(\mathrm{CH}), 128.6(\mathrm{CH}), 128.6(\mathrm{CH}), 127.8(\mathrm{CH}), 88.9(\mathrm{C}), 56.6(\mathrm{C}), 51.6\left(\mathrm{CH}_{2}\right), 45.6\left(\mathrm{CH}_{2}\right), 44.5$ $\left(\mathrm{CH}_{2}\right), 31.9$ (C), $31.7\left(\mathrm{CH}_{3}\right), 29.9\left(\mathrm{CH}_{3}\right)$ ppm (1 $\mathrm{CH}_{\text {Ar }}$ missing due to overlapping signals). HRMS (ESI): $m / z$ calculated for $\mathrm{C}_{34} \mathrm{H}_{38} \mathrm{~N}_{5} \mathrm{O}_{2}[\mathrm{M}+\mathrm{H}]^{+}=548.3026$, found $=548.3046$. M.p.: $143^{\circ} \mathrm{C}$.

\section{1,3-dibenzyl-5-[(5-(diethylamino)pentan-2-yl)amino]-7-phenylpyrimido[4,5- $d$ ]pyrimidine-} 2,4(1H,3H)-dione (3g) [JC782A]<smiles>CCN(CC)CCCC(C)Nc1nc(-c2ccccc2)nc2c1c(=O)n(Cc1ccccc1)c(=O)n2Cc1ccccc1</smiles>

The general procedure $C$ was applied using $N-(1,3-$ dibenzyluracil)benzimidamide (1a, $205 \mathrm{mg}, 0.5 \mathrm{mmol}, 1.0$ equiv) and 1,1,3,3-tetramethylbutyl isocyanide $(168 \mathrm{mg}, 1.0 \mathrm{mmol}, 2.0$ equiv). The compound was purified by manual flash chromatography using a cHex:EtOAc:NEt ${ }_{3}$ gradient (10:1:0.011:1:0.01). The title compound was isolated as an off-white solid (145 mg, 51\%). Unknown compound according to a Sci-finder search.

${ }^{1} \mathrm{H}$ NMR $\left(300 \mathrm{MHz}, \mathrm{CDCl}_{3}\right) \delta 8.92(\mathrm{~d}, J=8.1 \mathrm{~Hz}, 1 \mathrm{H}), 8.52-8.42(\mathrm{~m}, 2 \mathrm{H}), 7.58-7.42(\mathrm{~m}, 7 \mathrm{H}), 7.36-7.22$ $(\mathrm{m}, 6 \mathrm{H}), 5.58(\mathrm{~s}, 2 \mathrm{H}), 5.20(\mathrm{~s}, 2 \mathrm{H}), 4.66-4.51(\mathrm{~m}, 1 \mathrm{H}), 2.50(\mathrm{q}, J=7.0 \mathrm{~Hz}, 4 \mathrm{H}), 2.45(\mathrm{t}, J=6.5 \mathrm{~Hz}, 2 \mathrm{H})$, $1.76-1.50(\mathrm{~m}, 4 \mathrm{H}), 1.33(\mathrm{~d}, J=6.5 \mathrm{~Hz}, 3 \mathrm{H}), 0.99(\mathrm{t}, J=7.1 \mathrm{~Hz}, 6 \mathrm{H}) \mathrm{ppm} .{ }^{13} \mathrm{C} \mathrm{NMR}\left(101 \mathrm{MHz}, \mathrm{CDCl}_{3}\right) \delta$ 165.8 (C), 162.6 (C), 161.2 (C), 157.4 (C), 151.3 (C), 137.3 (C), 137.2 (C), 136.7 (C), 131.5 (CH), 128.9 $(\mathrm{CH}), 128.6(\mathrm{CH}), 128.5,128.4(\mathrm{CH}), 128.3(\mathrm{CH}), 127.6(\mathrm{CH}), 127.6(\mathrm{CH}), 88.4(\mathrm{C}), 52.8\left(\mathrm{CH}_{2}\right), 46.8\left(\mathrm{CH}_{2}\right)$, 
$46.7(\mathrm{CH}), 45.5\left(\mathrm{CH}_{2}\right), 44.4\left(\mathrm{CH}_{2}\right), 34.9\left(\mathrm{CH}_{2}\right), 23.9\left(\mathrm{CH}_{2}\right), 20.9\left(\mathrm{CH}_{3}\right), 11.6\left(\mathrm{CH}_{3}\right)$ ppm. HRMS (ESI): $\mathrm{m} / \mathrm{z}$ calculated for $\mathrm{C}_{35} \mathrm{H}_{41} \mathrm{~N}_{6} \mathrm{O}_{2}[\mathrm{M}+\mathrm{H}]^{+}=577.3286$, found $=577.3299$. M.p.: $76{ }^{\circ} \mathrm{C}$.

\section{1,3-dibenzyl-5-((2-morpholinoethyl)amino)-7-phenylpyrimido[4,5-d]pyrimidine-2,4(1H,3H)-dione} (3h) [BM1172]<smiles>O=c1c2c(NCCN3CCOCC3)nc(-c3ccccc3)nc2n(Cc2ccccc2)c(=O)n1Cc1ccccc1</smiles>

The general procedure $\mathrm{C}$ was applied using $\mathrm{N}-(1,3-$ dibenzyluracil)benzimidamide (1a, $205 \mathrm{mg}, 0.5 \mathrm{mmol}, 1.0$ equiv) and 2morpholinoethyl isocyanide (140 $\mu \mathrm{L}, 1.0 \mathrm{mmol}, 2.0$ equiv). The compound was purified by an automated flash chromatography system using a heptane / EtOAc gradient (from 100\% heptane to $50 \%$ ethyl acetate over $70 \mathrm{~min}, 35 \mathrm{~mL} / \mathrm{min}$ ). The title compound was isolated as a white solid (271 mg, 79\%). Unknown compound according to a Scifinder search.

$\mathbf{R}_{\mathbf{F}}=0.28$ (cHex : EtOAc: NEt $\left.{ }_{3} ; 9: 1: 0.01\right) .{ }^{1} \mathbf{H}$ NMR $\left(400 \mathrm{MHz}, \mathrm{CDCl}_{3}\right) \delta: 9.25$ (bt, $\left.J=5.0 \mathrm{~Hz}, 1 \mathrm{H}\right), 8.46$ $(\mathrm{d}, J=6.9 \mathrm{~Hz}, 2 \mathrm{H}), 7.54-7.45(\mathrm{~m}, 7 \mathrm{H}), 7.33-7.22(\mathrm{~m}, 6 \mathrm{H}), 5.58(\mathrm{~s}, 2 \mathrm{H}), 5.21(\mathrm{~s}, 2 \mathrm{H}), 3.83(\mathrm{q}, J=6.3 \mathrm{~Hz}$, $2 \mathrm{H}), 3.75(\mathrm{t}, J=4.6 \mathrm{~Hz}, 4 \mathrm{H}), 2.68(\mathrm{t}, J=6.5 \mathrm{~Hz}, 2 \mathrm{H}), 2.55(\mathrm{t}, J=4.4 \mathrm{~Hz}, 4 \mathrm{H}) \mathrm{ppm} .{ }^{13} \mathrm{C} \mathrm{NMR}(100 \mathrm{MHz}$, $\left.\mathrm{CDCl}_{3}\right) \delta: 165.9$ (C), 162.5 (C), 161.7 (C), 157.3 (C), 151.3 (C), 137.3 (C), 137.2 (C), 136.9 (C), 131.7 (CH), $129.0(\mathrm{CH}), 128.9(\mathrm{CH}), 128.7(\mathrm{CH}), 128.5(\mathrm{CH}), 128.5(\mathrm{CH}), 128.4(\mathrm{CH}), 127.8(\mathrm{CH}), 127.7(\mathrm{CH}), 88.9(\mathrm{C})$, $67.1\left(\mathrm{CH}_{2}\right), 57.2\left(\mathrm{CH}_{2}\right), 53.7\left(\mathrm{CH}_{2}\right), 45.6\left(\mathrm{CH}_{2}\right), 44.5\left(\mathrm{CH}_{2}\right), 37.9\left(\mathrm{CH}_{2}\right)$ ppm. HRMS (ESI): $\mathrm{m} / z$ calculated for $\mathrm{C}_{32} \mathrm{H}_{33} \mathrm{~N}_{6} \mathrm{O}_{3}[\mathrm{M}+\mathrm{H}]^{+}=549.2614$, found $=549.2610$. M.p.: $155^{\circ} \mathrm{C}$.

\section{1,3-dibenzyl-5-((3,4-dimethoxyphenethyl)amino)-7-phenylpyrimido[4,5-d]pyrimidine-2,4(1H,3H)-} dione (3i) [JC784A]<smiles>COc1ccc(CCNc2nc(-c3ccccc3)nc3c2c(=O)n(Cc2ccccc2)c(=O)n3Cc2ccccc2)cc1OC</smiles>

The general procedure $\mathrm{C}$ was applied using $\mathrm{N}-(1,3-$ dibenzyluracil)benzimidamide (1a, $205 \mathrm{mg}, 0.5 \mathrm{mmol}, 1.0$ equiv) and 4-(2-isocyanoethyl)-1,2-dimethoxybenzene (191 mg, 1.0 mmol, 2.0 equiv). The compound was purified by manual flash chromatography system using a cHex:EtOAc:NEt 3 (10:1:0.01) as eluent. The title compound was isolated as a white solid $(256 \mathrm{mg}$, 85\%). Unknown compound according to a Sci-finder search.

$\mathbf{R}_{\mathbf{F}}=0.37$ (cHex : EtOAc: $\left.\mathrm{NEt}_{3}=5: 1: 0.01\right) .{ }^{1} \mathbf{H}$ NMR $(300 \mathrm{MHz}$, $\left.\mathrm{CDCl}_{3}\right): \delta 9.15(\mathrm{bt}, J=6.5 \mathrm{~Hz}, 1 \mathrm{H}), 8.53(\mathrm{~d}, J=6.6 \mathrm{~Hz}, 2 \mathrm{H}), 7.62-7.44(\mathrm{~m}, 7 \mathrm{H}), 7.39-7.26(\mathrm{~m}, 6 \mathrm{H}), 6.85$ $(\mathrm{m}, 3 \mathrm{H}), 5.61(\mathrm{~s}, 2 \mathrm{H}), 5.22(\mathrm{~s}, 2 \mathrm{H}), 3.96(\mathrm{q}, J=6.9 \mathrm{~Hz}, 2 \mathrm{H}), 3.89(\mathrm{~s}, 6 \mathrm{H}), 2.99(\mathrm{t}, J=7.3 \mathrm{~Hz}, 2 \mathrm{H}) \mathrm{ppm} .{ }^{13} \mathrm{C}$ NMR $\left(101 \mathrm{MHz}, \mathrm{CDCl}_{3}\right) \delta 165.9$ (C), 162.5 (C), 161.7 (C), 157.3 (C), 151.2 (C), 149.0 (C), 147.8 (C), 137.2 (C), $137.1(\mathrm{C}), 136.7(\mathrm{C}), 131.6(\mathrm{CH}), 131.6(\mathrm{C}), 129.0(\mathrm{CH}), 128.7(\mathrm{CH}), 128.6(\mathrm{CH}), 128.5(2 \mathrm{CH}), 128.4$ $(\mathrm{CH}), 127.7(\mathrm{CH}), 127.6(\mathrm{CH}), 120.7(\mathrm{CH}), 112.2(\mathrm{CH}), 111.5(\mathrm{CH}), 88.7(\mathrm{C}), 56.0\left(\mathrm{CH}_{3}\right), 55.8\left(\mathrm{CH}_{3}\right), 45.6$ $\left(\mathrm{CH}_{2}\right), 44.5\left(\mathrm{CH}_{2}\right), 42.8\left(\mathrm{CH}_{2}\right), 35.4\left(\mathrm{CH}_{2}\right)$ ppm. HRMS (ESI): $\mathrm{m} / z$ calculated for $\mathrm{C}_{36} \mathrm{H}_{33} \mathrm{~N}_{5} \mathrm{O}_{4} \mathrm{Na}[\mathrm{M}+\mathrm{Na}]^{+}=$ 622.2425 , found $=622.2393$. M.p.: $182{ }^{\circ} \mathrm{C}$.

\section{1,3-dibenzyl-5-((2,6-dimethylphenyl)amino)-7-phenylpyrimido[4,5-d]pyrimidine-2,4(1H,3H)-dione} (3j) [HL064/BM1174/BM1160]<smiles>Cc1cccc(C)c1Nc1nc(-c2ccccc2)nc2c1c(=O)n(Cc1ccccc1)c(=O)n2Cc1ccccc1</smiles>

The general procedure $\mathrm{C}$ was applied using $\mathrm{N}$-(1,3dibenzyluracil)benzimidamide (1a, $205 \mathrm{mg}, 0.5 \mathrm{mmol}, 1.0$ equiv) and 2,6dimethylphenyl isocyanide ( $131 \mathrm{mg}, 1.0 \mathrm{mmol}, 2.0$ equiv). The compound was purified by an automated flash chromatography system using a heptane / EtOAc gradient (from 100\% heptane to 10\% EtOAc over $20 \mathrm{~min}, 35$ $\mathrm{mL} / \mathrm{min}$ ). The title compound was isolated as a white solid (214 $\mathrm{mg}, 79 \%$ ). Unknown compound according to a Sci-finder search.

$\mathbf{R}_{\mathbf{F}}=0.12$ (cHex : EtOAc: $\left.\mathrm{NEt}_{3} ; 9: 1: 0.01\right) .{ }^{1} \mathbf{H} \mathbf{~ N M R}\left(400 \mathrm{MHz}, \mathrm{CDCl}_{3}\right) \delta: 10.35$ (bs, $1 \mathrm{H}), 8.22(\mathrm{~d}, J=7.2 \mathrm{~Hz}, 2 \mathrm{H}), 7.59(\mathrm{~d}, J=7.0 \mathrm{~Hz}, 2 \mathrm{H}), 7.53(\mathrm{~d}, J=7.0 \mathrm{~Hz}, 2 \mathrm{H}), 7.46-7.42(\mathrm{~m}, 1 \mathrm{H}), 7.38$ - $7.26(\mathrm{~m}, 8 \mathrm{H}), 7.20$ - $7.14(\mathrm{~m}, 3 \mathrm{H}), 5.60(\mathrm{~s}, 2 \mathrm{H}), 5.26(\mathrm{~s}, 2 \mathrm{H}), 2.24(\mathrm{~s}, 6 \mathrm{H}) \mathrm{ppm} .{ }^{13} \mathrm{C} \mathrm{NMR}\left(100 \mathrm{MHz}, \mathrm{CDCl}_{3}\right)$ 
$\delta: 166.2(\mathrm{C}), 163.1(\mathrm{C}), 161.1(\mathrm{C}), 157.7$ (C), 151.4 (C), 137.2 (C), 137.1 (C), 136.8 (C), 135.6 (C), 135.1(C), 131.8 (CH), $129.2(\mathrm{CH}), 129.1(\mathrm{CH}), 129.0(\mathrm{CH}), 128.7(\mathrm{CH}), 128.7(\mathrm{CH}), 128.5(\mathrm{CH}), 128.2(\mathrm{CH}), 127.9$ $(\mathrm{CH}), \quad 127.9(\mathrm{CH}), \quad 127.2(\mathrm{CH}), \quad 89.0 \quad(\mathrm{C}), 45.8\left(\mathrm{CH}_{2}\right), 44.8\left(\mathrm{CH}_{2}\right), \quad 18.9\left(\mathrm{CH}_{3}\right)$ ppm. HRMS (ESI): $m / z$ calculated for $\mathrm{C}_{34} \mathrm{H}_{30} \mathrm{~N}_{5} \mathrm{O}_{2}[\mathrm{M}+\mathrm{H}]^{+}=540.2400$, found $=540.2408$. M.p.: $213^{\circ} \mathrm{C}$.

\section{Methyl 2-((6,8-dibenzyl-5,7-dioxo-2-phenyl-5,6,7,8-tetrahydropyrimido[4,5-d]pyrimidin-4-yl)} amino)benzoate $(3 \mathrm{~m})$ [JC803B/JC958]<smiles>CC(=O)c1ccccc1Nc1nc(-c2ccccc2)nc2c1c(=O)n(Cc1ccccc1)c(=O)n2Cc1ccccc1</smiles>

The general procedure $C$ was applied using $\mathrm{N}-(1,3-$ dibenzyluracil)benzimidamide (1a, $205 \mathrm{mg}, 0.5 \mathrm{mmol}, 1.0$ equiv) and methyl 2-isocyanobenzoate ( $161 \mathrm{mg}, 1.0 \mathrm{mmol}, 2.0$ equiv). The compound was purified by manual flash chromatography using cHex:EtOAc:NEt 3 (10:1:0.01) as eluent. The title compound was isolated as a white solid (56 $\mathrm{mg}, 21 \%)$. Unknown compound according to a Sci-finder search.

$\mathbf{R}_{\mathrm{F}}=0.62$ (cHex : EtOAc $\left.=4: 1\right) .{ }^{1} \mathbf{H}$ NMR $\left(300 \mathrm{MHz}, \mathrm{CDCl}_{3}\right) \delta 12.44(\mathrm{~s}, 1 \mathrm{H})$, $8.47(\mathrm{~d}, J=8.3 \mathrm{~Hz}, 1 \mathrm{H}), 8.41(\mathrm{~d}, J=7.2 \mathrm{~Hz}, 2 \mathrm{H}), 8.07(\mathrm{dd}, J=7.9,1.7 \mathrm{~Hz}, 1 \mathrm{H})$, $7.63(\mathrm{t}, J=7.4 \mathrm{~Hz}, 1 \mathrm{H}), 7.56-7.45(\mathrm{~m}, J=9.2 \mathrm{~Hz}, 5 \mathrm{H}), 7.47(\mathrm{t}, J=7.4 \mathrm{~Hz}, 2 \mathrm{H})$, 7.35 - $7.21(\mathrm{~m}, 7 \mathrm{H}), 5.62(\mathrm{~s}, 2 \mathrm{H}), 5.32(\mathrm{~s}, 2 \mathrm{H}), 3.96(\mathrm{~s}, 3 \mathrm{H}) \mathrm{ppm} .{ }^{13} \mathrm{C} \mathrm{NMR}\left(126 \mathrm{MHz}, \mathrm{CDCl}_{3}\right) \delta 167.1(\mathrm{C})$, 165.7 (C), 161.9 (C), 159.7 (C), 157.7 (C), 151.1 (C), 139.0 (C), 136.9 (C), 136.8 (C), 136.6 (C), 132.6 (CH), $131.9(\mathrm{CH}), 131.1(\mathrm{CH}), 129.1(\mathrm{CH}), 128.9(\mathrm{CH}), 128.5(\mathrm{CH}), 128.5(\mathrm{CH}), 128.5(\mathrm{CH}), 128.4(\mathrm{CH}), 127.7$ $(\mathrm{CH}), 127.7(\mathrm{CH}), 124.9(\mathrm{CH}), 123.8(\mathrm{CH}), 120.7(\mathrm{C}), 90.3(\mathrm{C}), 52.5\left(\mathrm{CH}_{3}\right), 45.7\left(\mathrm{CH}_{2}\right), 44.5\left(\mathrm{CH}_{2}\right)$ ppm. HRMS (ESI): $m / z$ calculated for $\mathrm{C}_{34} \mathrm{H}_{28} \mathrm{~N}_{5} \mathrm{O}_{4}[\mathrm{M}+\mathrm{H}]^{+}=570.2136$, found $=570.2151$.

\section{1,3-dibenzyl-5-(tert-butylamino)-7-(4-chlorophenyl)pyrimido[4,5- $d$ ]pyrimidine-2,4(1H,3H)-dione}

(4a) [HL116]<smiles>CC(C)(C)Nc1nc(-c2ccc(Cl)cc2)nc2c1c(=O)n(Cc1ccccc1)c(=O)n2Cc1ccccc1</smiles>

The general procedure $\mathrm{C}$ was applied using 4-chloro- $\mathrm{N}$-(1,3-dibenzyl2,6-dioxo-1,2,3,6-tetrahydropyrimidin-4-yl)benzimidamide (1), 223 $\mathrm{mg}, 0.5 \mathrm{mmol}, 1.0$ equiv) and tert-butyl isocyanide $(2 \mathrm{a}, 119 \mu \mathrm{L}, 1.0$ mmol, 2.0 equiv). The compound was purified by manual flash chromatography using cHex:EtOAc:NEt 3 (25:1:0.01) as eluent. The title compound was isolated as a white solid (234 mg, 89\%). Unknown compound according to a Sci-finder search.

$\mathbf{R}_{\mathbf{F}}=0.45$ (cHex:EtOAc:NEt $\left.{ }_{3} ; 15: 1: 0.01\right) .{ }^{1} \mathbf{H}$ NMR (300 MHz, $\left.\mathrm{CDCl}_{3}\right): \delta$

9.24 (bs, 1H), $8.38(\mathrm{~d}, J=8.5 \mathrm{~Hz}, 2 \mathrm{H}), 7.58-7.39(\mathrm{~m}, 6 \mathrm{H}), 7.38-7.17(\mathrm{~m}, 6 \mathrm{H}), 5.56(\mathrm{~s}, 2 \mathrm{H}), 5.20(\mathrm{~s}, 2 \mathrm{H})$, $1.60(\mathrm{~s}, 9 \mathrm{H}) \mathrm{ppm} .{ }^{13} \mathrm{C}$ NMR $\left(126 \mathrm{MHz}, \mathrm{CDCl}_{3}\right): \delta 164.1$ (C), 162.7 (C), 161.2 (C), 157.3 (C), 151.1 (C), 137.8 (C), $137.1(\mathrm{C}), 136.6(\mathrm{C}), 136.0(\mathrm{C}), 130.2(\mathrm{CH}), 128.6(\mathrm{CH}), 128.6(\mathrm{CH}), 128.5(\mathrm{CH}), 128.5(\mathrm{CH}), 127.6$ (CH), 88.8 (C), 52.6 (C), $45.5\left(\mathrm{CH}_{2}\right), 44.4\left(\mathrm{CH}_{2}\right), 29.0\left(\mathrm{CH}_{3}\right)$ ppm. HRMS (ESI): $m / z$ calculated for $\mathrm{C}_{30} \mathrm{H}_{29} \mathrm{~N}_{5} \mathrm{O}_{2} \mathrm{Cl}[\mathrm{M}+\mathrm{H}]^{+}=526.2003$, found $=526.2004$. M.p.: $207^{\circ} \mathrm{C}$.

\section{1,3-dibenzyl-7-(4-chlorophenyl)-5-((2,6-dimethylphenyl)amino)pyrimido[4,5-d]pyrimidine- 2,4(1H,3H)-dione (4b) [JC900A]}<smiles>Cc1cccc(C)c1Nc1nc(-c2ccc(Cl)cc2)nc2c1c(=O)n(Cc1ccccc1)c(=O)n2Cc1ccccc1</smiles>

The general procedure $C$ was applied using $N$-(1,3-dibenzyl-2,6-dioxo1,2,3,6-tetrahydropyrimidin-4-yl)-4-chloro-benzimidamide (1b, 223 $\mathrm{mg}, 0.5 \mathrm{mmol}, 1.0$ equiv) and 2,6-dimethylphenyl isocyanide (131 mg, $1.0 \mathrm{mmol}, 2.0$ equiv). The compound was purified by manual flash chromatography using cHex:EtOAc:NEt 3 (10:1:0.01) as eluent. The title compound was isolated as a white solid (290 mg, 99\%). Unknown compound according to a Sci-finder search.

$\mathbf{R}_{\mathbf{F}}=0.22$ (cHex : EtOAc: NEt3 ; 9:1:0.01). ${ }^{1} \mathbf{H}$ NMR $\left(300 \mathrm{MHz}, \mathrm{CDCl}_{3}\right): \delta$ 10.39(bs, 1H), $8.15(\mathrm{~d}, J=8.6 \mathrm{~Hz}, 2 \mathrm{H}), 7.61-7.51(\mathrm{~m}, 4 \mathrm{H}), 7.40-7.25(\mathrm{~m}, 8 \mathrm{H}), 7.24-7.12(\mathrm{~m}, 3 \mathrm{H}), 5.59$ (s, 2H), $5.27(\mathrm{~s}, 2 \mathrm{H}), 2.24(\mathrm{~s}, 6 \mathrm{H}) \mathrm{ppm} .{ }^{13} \mathrm{C} \mathrm{NMR}\left(101 \mathrm{MHz}, \mathrm{CDCl}_{3}\right) \delta 165.1$ (C), 162.8 (C), 160.9 (C), 157.5 (C), 151.2 (C), 138.0 (C), 136.9 (C), 136.6 (C), 135.42 (C) 135.41 (C), 134.8 (C), 130.3 (CH), 129.0 (CH), $128.7(\mathrm{CH}), 128.6(\mathrm{CH}), 128.55(\mathrm{CH}), 128.54(\mathrm{CH}), 128.0(\mathrm{CH}), 127.79(\mathrm{CH}), 127.78(\mathrm{CH}), 127.3(\mathrm{CH}), 88.9$ 
(C), $45.7\left(\mathrm{CH}_{2}\right), 44.7\left(\mathrm{CH}_{2}\right), 18.8\left(\mathrm{CH}_{3}\right)$ ppm. HRMS (ESI): $\mathrm{m} / z$ calculated for $\mathrm{C}_{34} \mathrm{H}_{29} \mathrm{~N}_{5} \mathrm{O}_{2} \mathrm{Cl}[\mathrm{M}+\mathrm{H}]^{+}=$ 574.2004, found $=574.2015$. M.p.: $229^{\circ} \mathrm{C}$.

1,3-dibenzyl-5-(butylamino)-7-(4-chlorophenyl)pyrimido[4,5- $d$ ]pyrimidine-2,4(1H,3H)-dione [JC900B]<smiles>CCCCNc1nc(-c2ccc(Cl)cc2)nc2c1c(=O)n(Cc1ccccc1)c(=O)n2Cc1ccccc1</smiles>

The general procedure $\mathrm{C}$ was applied using $\mathrm{N}$-(1,3-dibenzyl-2,6-dioxo1,2,3,6-tetrahydropyrimidin-4-yl)-4-chlorobenzimidamide (1), 223 $\mathrm{mg}, 0.5 \mathrm{mmol}, 1.0$ equiv) and $n$-butyl isocyanide (105 $\mu \mathrm{L}, 1.0 \mathrm{mmol}$, 2.0 equiv). The compound was purified by manual flash chromatography using cHex:EtOAc:NEt ${ }_{3}$ (10:1:0.01) as eluent. The title compound was isolated as a white solid ( $260 \mathrm{mg}, 99 \%)$. Unknown compound according to a Sci-finder search.

$\mathbf{R}_{\mathbf{F}}=0.21$ (cHex : EtOAc: NEt $\left.{ }_{3} ; 9: 1: 0.01\right) .{ }^{1} \mathbf{H}$ NMR $\left(300 \mathrm{MHz}, \mathrm{CDCl}_{3}\right): \delta 9.09$ (bt, $\left.J=5.7 \mathrm{~Hz}, 1 \mathrm{H}\right), 8.43$ $(\mathrm{d}, J=8.7 \mathrm{~Hz}, 2 \mathrm{H}), 7.65-7.40(\mathrm{~m}, 6 \mathrm{H}), 7.40-7.22(\mathrm{~m}, 6 \mathrm{H}), 5.58(\mathrm{~s}, 2 \mathrm{H}), 5.23(\mathrm{~s}, 2 \mathrm{H}), 3.72(\mathrm{q}, J=6.7 \mathrm{~Hz}$, $2 \mathrm{H}), 1.72(\mathrm{p}, J=7.2 \mathrm{~Hz}, 2 \mathrm{H}), 1.59-1.40(\mathrm{~m}, 2 \mathrm{H}), 1.02(\mathrm{t}, J=7.3 \mathrm{~Hz}, 3 \mathrm{H}) \mathrm{ppm} .{ }^{13} \mathrm{C}$ NMR $\left(101 \mathrm{MHz}, \mathrm{CDCl}_{3}\right)$ $\delta 164.9$ (C), 162.6 (C), 161.7 (C), 157.3 (C), 151.2 (C), 137.9 (C), 137.1 (C), 136.7 (C), 135.8 (C), 130.3 $(\mathrm{CH}), 128.7(\mathrm{CH}), 128.6(\mathrm{CH}), 128.51(\mathrm{CH}), 128.49(\mathrm{CH}), 127.7(\mathrm{CH}), 127.7(\mathrm{CH}), 88.6(\mathrm{C}), 45.6\left(\mathrm{CH}_{2}\right), 44.5$ $\left(\mathrm{CH}_{2}\right), 40.7\left(\mathrm{CH}_{2}\right), 31.5\left(\mathrm{CH}_{2}\right), 20.2\left(\mathrm{CH}_{2}\right), 13.8\left(\mathrm{CH}_{3}\right) \mathrm{ppm}$. HRMS (ESI): $\mathrm{m} / z$ calculated for $\mathrm{C}_{30} \mathrm{H}_{28} \mathrm{~N}_{5} \mathrm{O}_{2} \mathrm{ClNa}$ $[\mathrm{M}+\mathrm{Na}]^{+}=548.1824$, found $=548.1802$. M.p.: $154^{\circ} \mathrm{C}$.

\section{1,3-dibenzyl-5-(tert-butylamino)-7-(4-(trifluoromethyl)phenyl)pyrimido[4,5-d]pyrimidine-} 2,4(1H,3H)-dione (4d) [HL055]<smiles>CC(C)(C)Nc1nc(-c2ccc(C(F)(F)F)cc2)nc2c1c(=O)n(Cc1ccccc1)c(=O)n2Cc1ccccc1</smiles>

The general procedure $C$ was applied using $N$-(1,3-dibenzyl-2,6dioxo-1,2,3,6-tetrahydropyrimidin-4-yl)-4-(trifluoromethyl) benzimidamide (1c, $239 \mathrm{mg}, 0.5 \mathrm{mmol}, 1.0$ equiv) and tert-butyl isocyanide ( $119 \mu \mathrm{L}, 1.0 \mathrm{mmol}, 2.0$ equiv). The compound was purified by manual flash chromatography using cHex:EtOAc:NEt 3 (25:1:0.01) as eluent. The title compound was isolated as a white solid (174 mg, $62 \%)$. Unknown compound according to a Sci-finder search.

$\mathbf{R}_{\mathbf{F}}=0.44$ (cHex : EtOAc: $\left.\mathrm{NEt}_{3}=10: 1: 0.01\right) .{ }^{1} \mathbf{H}$ NMR $\left(300 \mathrm{MHz}, \mathrm{CDCl}_{3}\right):$ $\delta 9.29$ (br s, 1H), 8.54 (d, J=9.0 Hz, 2H), $7.75(\mathrm{~d}, J=9.0 \mathrm{~Hz}, 2 \mathrm{H}$ ), 7.52 (dd, $J=3.0,9.0 \mathrm{~Hz}, 2 \mathrm{H}$ ), 7.48 $(\mathrm{dd}, J=3.0,9.0 \mathrm{~Hz}, 2 \mathrm{H}), 7.35-7.25(\mathrm{~m}, 6 \mathrm{H}), 5.57(\mathrm{~s}, 2 \mathrm{H}), 5.21(\mathrm{~s}, 2 \mathrm{H}), 1.61(\mathrm{~s}, 9 \mathrm{H}) .{ }^{13} \mathrm{C}$ NMR $(126 \mathrm{MHz}$, $\mathrm{CDCl}_{3}$ ): $\delta 163.8$ (C), 162.7 (C), 161.3 (C), 157.3 (C), 151.1 (C), 140.8 (C), 137.0 (C), 136.5 (C), 132.9 (q, ${ }^{2}$ J $=33 \mathrm{~Hz}, \mathrm{C}), 129.1(\mathrm{CH}), 128.6(\mathrm{CH}), 128.5(\mathrm{CH}), 128.5(\mathrm{CH}), 128.4(\mathrm{CH}), 127.7(\mathrm{CH}), 127.7(\mathrm{CH}), 125.3(\mathrm{q}$, $\left.{ }^{3} \mathrm{~J}=5 \mathrm{~Hz}, \mathrm{CH}\right), 124.0\left(\mathrm{q},{ }^{1} \mathrm{~J}=270 \mathrm{~Hz}, \mathrm{C}\right), 89.1(\mathrm{C}), 52.8(\mathrm{C}), 45.6\left(\mathrm{CH}_{2}\right), 44.5\left(\mathrm{CH}_{2}\right), 29.0\left(\mathrm{CH}_{3}\right)$ ppm. HRMS (ESI): $m / z$ calculated for $\mathrm{C}_{31} \mathrm{H}_{29} \mathrm{~N}_{5} \mathrm{O}_{2} \mathrm{~F}_{3}[\mathrm{M}+\mathrm{H}]^{+}=560.2264$, found $=560.2268$. M.p.: $172{ }^{\circ} \mathrm{C}$.

\section{1,3-dibenzyl-5-(cyclohexylamino)-7-(4-(trifluoromethyl)phenyl)pyrimido[4,5-d]pyrimidine-} 2,4(1H,3H)-dione (4e) [HL057]<smiles>O=c1c2c(NC3CCCCC3)nc(-c3ccc(C(F)(F)F)cc3)nc2n(Cc2ccccc2)c(=O)n1Cc1ccccc1</smiles>

The general procedure $C$ was applied using $N$-(1,3-dibenzyl-2,6dioxo-1,2,3,6-tetrahydropyrimidin-4-yl)-4-(trifluoromethyl) benzimidamide (1c, $239 \mathrm{mg}, 0.5 \mathrm{mmol}, 1.0$ equiv) and cyclohexyl isocyanide ( $124 \mu \mathrm{L}, 1.0 \mathrm{mmol}, 2.0$ equiv). The compound was purified by manual flash chromatography using cHex:EtOAc:NEt 3 (50:1:0.01) as eluent. The title compound was isolated as a white solid (152 mg, $52 \%)$. Unknown compound according to a Sci-finder search.

$\mathbf{R}_{\mathbf{F}}=0.48$ (cHex :EtOAc: $\left.\mathrm{NEt}_{3}=10: 1: 0.01\right) .{ }^{1} \mathbf{H}$ NMR $\left(300 \mathrm{MHz}, \mathrm{CDCl}_{3}\right)$ : $\delta 9.07(\mathrm{~d}, J=9.0 \mathrm{~Hz}, 1 \mathrm{H}), 8.54(\mathrm{~d}, J=9.0 \mathrm{~Hz}, 2 \mathrm{H}), 7.74(\mathrm{~d}, J=9.0 \mathrm{~Hz}$, $2 \mathrm{H}), 7.52(\mathrm{~d}, J=9.0 \mathrm{~Hz}, 2 \mathrm{H}), 7.49(\mathrm{~d}, J=9.0 \mathrm{~Hz}, 2 \mathrm{H}), 7.37-7.27(\mathrm{~m}, 6 \mathrm{H}), 5.58(\mathrm{~s}, 2 \mathrm{H}), 5.22(\mathrm{~s}, 2 \mathrm{H}), 4.38-$ $4.27(\mathrm{~m}, 1 \mathrm{H}), 2.14-2.03(\mathrm{~m}, 2 \mathrm{H}), 1.89-1.78(\mathrm{~m}, 2 \mathrm{H}), 1.74-1.65(\mathrm{~m}, 1 \mathrm{H}), 1.60-1.50(\mathrm{~m}, 1 \mathrm{H}), 1.48-$ $1.24(\mathrm{~m}, 4 \mathrm{H})-.{ }^{13} \mathrm{C}$ NMR $\left(126 \mathrm{MHz}, \mathrm{CDCl}_{3}\right): \delta 164.5$ (C), 162.5 (C), 160.8 (C), 157.4 (C), 151.2 (C), 140.6 (C), 137.0 (C), 136.5 (C), 132.9 (q, $\left.{ }^{2} \mathrm{~J}=33 \mathrm{~Hz}, \mathrm{C}\right), 129.1(\mathrm{CH}), 128.6(\mathrm{CH}), 128.5(\mathrm{CH}), 128.5(\mathrm{CH}), 128.4$ 
(CH), $127.7(\mathrm{CH}), 127.7(\mathrm{CH}), 125.3\left(\mathrm{q},{ }^{3} \mathrm{~J}=5 \mathrm{~Hz}, \mathrm{CH}\right.$ ), 124.0 (q, $\left.{ }^{1} \mathrm{~J}=270 \mathrm{~Hz}, \mathrm{C}\right), 88.7$ (C), 49.8 (CH), 45.6 $\left(\mathrm{CH}_{2}\right), \quad 44.5\left(\mathrm{CH}_{2}\right), \quad 32.6\left(\mathrm{CH}_{2}\right), 25.6\left(\mathrm{CH}_{2}\right), 24.7\left(\mathrm{CH}_{2}\right) \quad$ ppm. HRMS (ESI): $m / z$ calculated for $\mathrm{C}_{33} \mathrm{H}_{31} \mathrm{~N}_{5} \mathrm{O}_{2} \mathrm{~F}_{3}[\mathrm{M}+\mathrm{H}]^{+}=586.2443$, found $=586.2424$. M.p.: $205^{\circ} \mathrm{C}$.

\section{1,3-dibenzyl-5-(benzylamino)-7-(4-(trifluoromethyl)phenyl)pyrimido[4,5-d]pyrimidine-2,4(1H,3H)- dione (4f) [HL056]}<smiles>O=c1c2c(NCc3ccccc3)nc(-c3ccc(C(F)(F)F)cc3)nc2n(Cc2ccccc2)c(=O)n1Cc1ccccc1</smiles>

The general procedure $C$ was applied using $N$-(1,3-dibenzyl-2,6dioxo-1,2,3,6-tetrahydropyrimidin-4-yl)-4-(trifluoromethyl) benzimidamide (1c, $239 \mathrm{mg}, 0.5 \mathrm{mmol}, 1.0$ equiv) and benzyl isocyanide ( $122 \mu \mathrm{L}, 1.0 \mathrm{mmol}, 2.0$ equiv). The compound was purified by manual flash chromatography using cHex:EtOAc:NEt 3 (25:1:0.01) as eluent. The title compound was isolated as a white solid (172 mg, $58 \%)$. Unknown compound according to a Sci-finder search.

$\mathbf{R}_{\mathbf{F}}=0.37$ (cHex : EtOAc: $\left.\mathrm{NEt}_{3}=10: 1: 0.01\right) .{ }^{1} \mathbf{H}$ NMR $\left(300 \mathrm{MHz}, \mathrm{CDCl}_{3}\right): \delta 9.49-9.43(\mathrm{~m}, 1 \mathrm{H}), 8.54(\mathrm{~d}, J=$ $6.0 \mathrm{~Hz}, 2 \mathrm{H}), 7.72(\mathrm{~d}, J=6.0 \mathrm{~Hz}, 2 \mathrm{H}), 7.52(\mathrm{dd}, J=3.0,9.0 \mathrm{~Hz}, 2 \mathrm{H}), 7.47(\mathrm{dd}, J=3.0,9.0 \mathrm{~Hz}, 2 \mathrm{H}), 7.43-7.27$ $(\mathrm{m}, 11 \mathrm{H}), 5.58(\mathrm{~s}, 2 \mathrm{H}), 5.21(\mathrm{~s}, 2 \mathrm{H}), 4.92(\mathrm{~d}, J=6.0 \mathrm{~Hz}, 2 \mathrm{H}) \mathrm{ppm} .{ }^{13} \mathrm{C} \mathrm{NMR}\left(126 \mathrm{MHz}, \mathrm{CDCl}_{3}\right): \delta 164.7(\mathrm{C})$, 162.5 (C), 161.7 (C), 157.4 (C), 151.1 (C), 140.4 (C), 138.1 (C), 136.8 (C), 136.5 (C), 133.0 (q, ${ }^{2} \mathrm{~J}=33 \mathrm{~Hz}$, C), $129.2(\mathrm{CH}), 128.8(\mathrm{CH}), 128.7(\mathrm{CH}), 128.5(\mathrm{CH}), 128.5(\mathrm{CH}), 128.5(\mathrm{CH}), 127.7(\mathrm{CH}), 127.7(\mathrm{CH}), 127.6$ $(\mathrm{CH}), 125.3\left(\mathrm{q},{ }^{3} \mathrm{~J}=5 \mathrm{~Hz}, \mathrm{CH}\right), 124.0\left(\mathrm{q},{ }^{1} \mathrm{~J}=270 \mathrm{~Hz}, \mathrm{C}\right), 89.1(\mathrm{C}), 45.7\left(\mathrm{CH}_{2}\right), 44.9\left(\mathrm{CH}_{2}\right), 44.6\left(\mathrm{CH}_{2}\right)$ ppm. HRMS (ESI): $\mathrm{m} / z$ calculated for $\mathrm{C}_{34} \mathrm{H}_{27} \mathrm{~N}_{5} \mathrm{O}_{2} \mathrm{~F}_{3}[\mathrm{M}+\mathrm{H}]^{+}=594.2112$, found $=594.2111$. M.p.: $182{ }^{\circ} \mathrm{C}$.

\section{1,3-dibenzyl-5-(tert-butylamino)-7-(4-methoxyphenyl)pyrimido[4,5-d]pyrimidine-2,4(1H,3H)-dione} (4g) [HL088]<smiles>COc1ccc(-c2nc(NC(C)(C)C)c3c(=O)n(Cc4ccccc4)c(=O)n(Cc4ccccc4)c3n2)cc1</smiles>

The general procedure $C$ was applied using $N$-(1,3-dibenzyl2,6-dioxo-1,2,3,6-tetrahydropyrimidin-4-yl)-4methoxybenzimidamide (1d, $220 \mathrm{mg}, 0.5 \mathrm{mmol}, 1.0$ equiv) and tertbutyl isocyanide ( $2 \mathrm{a}, 220 \mu \mathrm{L}, 1.5 \mathrm{mmol}, 3.0$ equiv). The compound was purified by manual flash chromatography using cHex:EtOAc:NEt ${ }_{3}$ (gradient $=$ 40:1:0.01 - 10:1:0.01) as eluent. The title compound was isolated as a white solid (178 $\mathrm{mg}, 68 \%$ ). Unknown compound according to a Sci-finder search.

$\mathbf{R}_{\mathbf{F}}=0.25$ (cHex : EtOAc: $\left.\mathrm{NEt}_{3}=15: 1: 0.01\right) .{ }^{1} \mathrm{H} \mathrm{NMR}\left(300 \mathrm{MHz}, \mathrm{CDCl}_{3}\right): \delta 9.17(\mathrm{~s}, 1 \mathrm{H}), 8.43$ (d, J $=9.0 \mathrm{~Hz}$, $2 \mathrm{H}), 7.54(\mathrm{~d}, J=6.0 \mathrm{~Hz}, 2 \mathrm{H}), 7.46(\mathrm{~d}, J=6.0 \mathrm{~Hz}, 2 \mathrm{H}), 7.37-7.21(\mathrm{~m}, 6 \mathrm{H}), 7.00(\mathrm{~d}, J=6.0 \mathrm{~Hz}, 2 \mathrm{H}), 5.57(\mathrm{~s}$, 2H), 5.19 (s, 2H), $3.89(\mathrm{~s}, 3 \mathrm{H}), 1.61(\mathrm{~s}, 9 \mathrm{H}) \mathrm{ppm} .{ }^{13} \mathrm{C}$ NMR $\left(150.90 \mathrm{MHz}, \mathrm{CDCl}_{3}\right): \delta 164.8$ (C), 162.7 (C), $162.6(\mathrm{C}), 161.2$ (C), 157.2 (C), 151.3 (C), 137.3 (C), 136.7 (C), $130.8(\mathrm{CH}), 130.1(\mathrm{C}), 128.6(\mathrm{CH}), 128.5$ $(\mathrm{CH}), 128.5(\mathrm{CH}), 128.4(\mathrm{CH}), 127.6(\mathrm{CH}), 127.6(\mathrm{CH}), 113.7(\mathrm{CH}), 88.4(\mathrm{C}), 55.4\left(\mathrm{CH}_{3}\right), 52.5(\mathrm{C}), 45.3\left(\mathrm{CH}_{2}\right)$, $44.3\left(\mathrm{CH}_{2}\right), 29.1\left(\mathrm{CH}_{3}\right)$ ppm. M.p.: $237^{\circ} \mathrm{C}$.

\section{1,3-dibenzyl-5-((2,6-dimethylphenyl)amino)-7-(4-methoxyphenyl)pyrimido[4,5- $d$ ]pyrimidine- 2,4(1H,3H)-dione (4h) [HL090]}<smiles>COc1ccc(-c2nc(Nc3c(C)cccc3C)c3c(=O)n(Cc4ccccc4)c(=O)n(Cc4ccccc4)c3n2)cc1</smiles>

The general procedure $\mathrm{C}$ was applied using $\mathrm{N}$-(1,3-dibenzyl-2,6dioxo-1,2,3,6-tetrahydropyrimidin-4-yl)-4-methoxybenzimidamide (1d, $220 \mathrm{mg}, 0.5 \mathrm{mmol}, 1.0$ equiv) and 2,6-dimethylphenylisocyanide (131 $\mathrm{mg}, 1.0 \mathrm{mmol}, 2.0$ equiv). The compound was purified by manual flash chromatography using cHex:EtOAc:NEt 3 (15:1:0.01) as eluent. The title compound was isolated as an off-white solid (224 $\mathrm{mg}, 79 \%)$. Unknown compound according to a Sci-finder search.

$\mathbf{R}_{\mathbf{F}}=0.39$ (cHex : EtOAc: $\left.\mathrm{NEt}_{3}=7: 1: 0.01\right) .{ }^{1} \mathbf{H}$ NMR $\left(300 \mathrm{MHz}, \mathrm{CDCl}_{3}\right)$ : $\delta 10.33(\mathrm{~s}, 1 \mathrm{H}), 8.19(\mathrm{~d}, J=6.0 \mathrm{~Hz}, 2 \mathrm{H}), 7.60(\mathrm{~d}, J=6.0 \mathrm{~Hz}, 2 \mathrm{H}), 7.54$ $(\mathrm{d}, J=6.0 \mathrm{~Hz}, 2 \mathrm{H}), 7.40-7.27(\mathrm{~m}, 6 \mathrm{H}), 7.21-7.12(\mathrm{~m}, 3 \mathrm{H}), 6.88(\mathrm{~d}, J=6.0 \mathrm{~Hz}, 2 \mathrm{H}), 5.60(\mathrm{~s}, 2 \mathrm{H}), 5.26(\mathrm{~s}$, 2H), 3.83 (s, 3H), 2.24 (s, 6H) ppm. ${ }^{13} \mathrm{C}$ NMR (126 MHz, CDCl 3 ): $\delta 165.7$ (C), 162.9 (C), 162.7 (C), 160.8 (C), 157.4 (C), 151.3 (C), 137.1 (C), 136.7 (C), 135.4 (C), 135.0 (C), 130.9 (CH), 129.5 (C), 128.9 (CH), 
128.8 (CH), $128.50(\mathrm{CH}), 128.49(\mathrm{CH}), 127.9(\mathrm{CH}), 127.7(\mathrm{CH}), 127.7(\mathrm{CH}), 126.9(\mathrm{CH}), 113.7(\mathrm{CH}), 88.4$ (C), $55.4\left(\mathrm{CH}_{3}\right), \quad 45.5 \quad\left(\mathrm{CH}_{2}\right), \quad 44.6 \quad\left(\mathrm{CH}_{2}\right), \quad 18.8 \quad\left(\mathrm{CH}_{3}\right) \quad$ ppm. HRMS (ESI): $m / z$ calculated for $\mathrm{C}_{35} \mathrm{H}_{32} \mathrm{~N}_{5} \mathrm{O}_{3}[\mathrm{M}+\mathrm{H}]^{+}=570.2500$, found $=570.2500$. M.p.: $237^{\circ} \mathrm{C}$.

\section{1,3-dibenzyl-5-(cyclohexylamino)-7-(4-(trifluoromethyl)phenyl)pyrimido[4,5- $d$ ]pyrimidine- 2,4(1H,3H)-dione (4i) [HL089]}<smiles>COc1ccc(-c2nc(NC3CCCCC3)c3c(=O)n(Cc4ccccc4)c(=O)n(Cc4ccccc4)c3n2)cc1</smiles>

The general procedure $\mathrm{C}$ was applied using $\mathrm{N}$-(1,3-dibenzyl-2,6dioxo-1,2,3,6-tetrahydropyrimidin-4-yl)-4-methoxybenzimidamide (1d, $220 \mathrm{mg}, 0.5 \mathrm{mmol}, 1.0$ equiv) and cyclohexyl isocyanide ( $124 \mu \mathrm{L}$, $1.0 \mathrm{mmol}, 2.0$ equiv). The compound was purified by manual flash chromatography using cHex:EtOAc:NEt ${ }_{3}$ (gradient $=20: 1: 0.01-$ 10:1:0.01) as eluent. The title compound was isolated as an offwhite solid ( $211 \mathrm{mg}, 77 \%$ ). Unknown compound according to a Scifinder search.

$\mathbf{R}_{\mathbf{F}}=0.41$ (cHex : EtOAc: NEt $\left.t_{3}=7: 1: 0.01\right) .{ }^{1} \mathbf{H}$ NMR $\left(300 \mathrm{MHz}, \mathrm{CDCl}_{3}\right): \delta 8.96(\mathrm{~d}, J=9.0,1 \mathrm{H}), 8.42(\mathrm{~d}, J=$ $9.0,2 \mathrm{H}), 7.53(\mathrm{~d}, J=9.0 \mathrm{~Hz}, 2 \mathrm{H}), 7.46(\mathrm{~d}, J=9.0 \mathrm{~Hz}, 2 \mathrm{H}), 7.37-7.20(\mathrm{~m}, 6 \mathrm{H}), 6.98(\mathrm{~d}, J=9.0 \mathrm{~Hz}, 2 \mathrm{H}), 5.56$ (s, 2H), $5.19(\mathrm{~s}, 2 \mathrm{H}), 4.39-4.22(\mathrm{~m}, 1 \mathrm{H}), 3.89(\mathrm{~s}, 3 \mathrm{H}), 2.15-1.99(\mathrm{~m}, 2 \mathrm{H}), 1.89-1.75(\mathrm{~m}, 2 \mathrm{H}), 1.73-1.62$ $(\mathrm{m}, 1 \mathrm{H}), 1.55-1.22(\mathrm{~m}, 5 \mathrm{H}) .{ }^{13} \mathrm{C} \mathrm{NMR}\left(126 \mathrm{MHz}, \mathrm{CDCl}_{3}\right): \delta 165.5(\mathrm{C}), 162.6(\mathrm{C}), 162.6(\mathrm{C}), 160.7(\mathrm{C})$, $157.3(\mathrm{C}), 151.3(\mathrm{C}), 137.2(\mathrm{C}), 136.8(\mathrm{C}), 130.8(\mathrm{CH}), 130.0(\mathrm{C}), 128.6(\mathrm{CH}), 128.5(\mathrm{CH}), 128.5(\mathrm{CH}), 128.4$ $(\mathrm{CH}), 127.6(\mathrm{CH}), 127.5(\mathrm{CH}), 113.7(\mathrm{CH}), 88.0(\mathrm{C}), 55.4\left(\mathrm{CH}_{3}\right), 49.6(\mathrm{CH}), 45.4\left(\mathrm{CH}_{2}\right), 44.3\left(\mathrm{CH}_{2}\right), 32.7$ $\left(\mathrm{CH}_{2}\right), 25.7\left(\mathrm{CH}_{2}\right), 24.8\left(\mathrm{CH}_{2}\right)$ ppm. HRMS (ESI): $\mathrm{m} / z$ calculated for $\mathrm{C}_{33} \mathrm{H}_{34} \mathrm{~N}_{5} \mathrm{O}_{3}[\mathrm{M}+\mathrm{H}]^{+}=548.2656$, found $=$ 548.2632. M.p.: $205^{\circ} \mathrm{C}$.

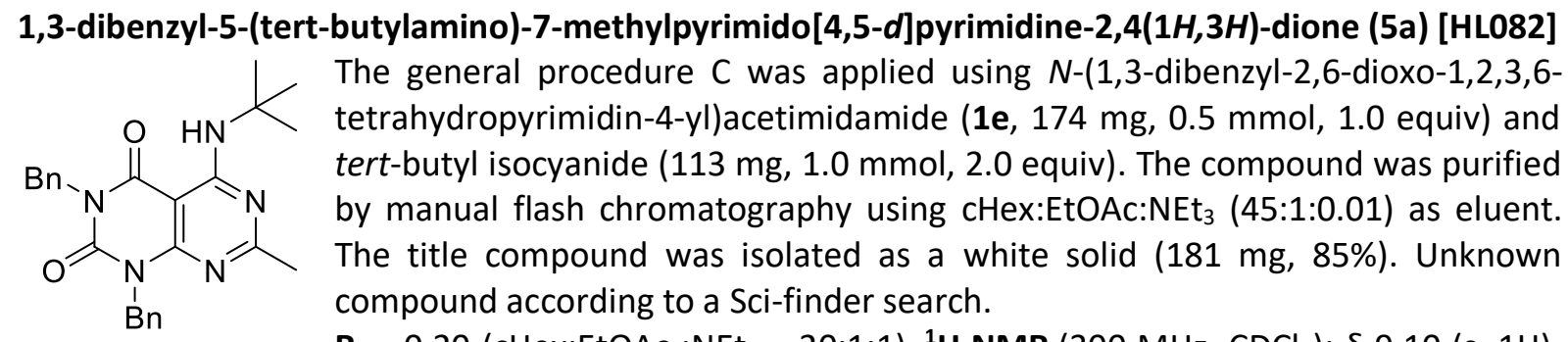
$\mathbf{R}_{\mathbf{F}}=0.20$ (cHex:EtOAc : $\left.\mathrm{NEt}_{3}=20: 1: 1\right) .{ }^{1} \mathbf{H}$ NMR $\left(300 \mathrm{MHz}, \mathrm{CDCl}_{3}\right): \delta 9.10(\mathrm{~s}, 1 \mathrm{H})$, $7.53(\mathrm{~d}, J=6.0 \mathrm{~Hz}, 2 \mathrm{H}), 7.47(\mathrm{~d}, J=6.0 \mathrm{~Hz}, 2 \mathrm{H}), 7.37-7.23(\mathrm{~m}, 6 \mathrm{H}), 5.46(\mathrm{~s}, 2 \mathrm{H}), 5.20(\mathrm{~s}, 2 \mathrm{H}), 2.51(\mathrm{~s}$, 3H), $1.54(\mathrm{~s}, 9 \mathrm{H})$ ppm. ${ }^{13} \mathrm{C} \mathrm{NMR}\left(150.90 \mathrm{MHz}, \mathrm{CDCl}_{3}\right): \delta 170.4$ (C), 162.8 (C), 161.1 (C), 156.9 (C), 151.1 (C), $137.1(\mathrm{C}), 136.7(\mathrm{C}), 128.9(\mathrm{CH}), 128.5(\mathrm{CH}), 128.4(\mathrm{CH}), 128.3(\mathrm{CH}), 127.5(\mathrm{CH}), 127.5(\mathrm{CH}), 88.1(\mathrm{C})$, 52.5 (C), $45.0\left(\mathrm{CH}_{2}\right), 44.3\left(\mathrm{CH}_{2}\right), 28.9\left(\mathrm{CH}_{3}\right), 27.0\left(\mathrm{CH}_{3}\right) \mathrm{ppm}$. HRMS (ESI): $m / z$ calculated for $\mathrm{C}_{25} \mathrm{H}_{28} \mathrm{~N}_{5} \mathrm{O}_{2}[\mathrm{M}+\mathrm{H}]^{+}=430.2238$, found $=430.2238$. M.p.: $100^{\circ} \mathrm{C}$.

\section{1,3-dibenzyl-5-(isopropylamino)-7-methylpyrimido[4,5-d]pyrimidine-2,4(1H,3H)-dione (5b) [HL131]}<smiles>Cc1nc(NC(C)C)c2c(=O)n(Cc3ccccc3)c(=O)n(Cc3ccccc3)c2n1</smiles>
The general procedure $C$ was applied using $N$-(1,3-dibenzyl-2,6-dioxo-1,2,3,6tetrahydropyrimidin-4-yl)acetimidamide (1e, $174 \mathrm{mg}, 0.5 \mathrm{mmol}, 1.0$ equiv) and isopropyl isocyanide ( $94 \mu \mathrm{L}, 1.0 \mathrm{mmol}, 2.0$ equiv). The compound was purified by manual flash chromatography using cHex:EtOAc (30:1) as eluent. The title compound was isolated as a white solid (56 mg, 30\%). Unknown compound according to a Sci-finder search.

$\mathbf{R}_{\mathbf{F}}=0.31$ (cHex : EtOAc : NEt $\left.{ }_{3}=10: 1: 1\right) .{ }^{1} \mathbf{H}$ NMR $\left(300 \mathrm{MHz}, \mathrm{CDCl}_{3}\right): \delta 8.88-8.76$

$(\mathrm{m}, 1 \mathrm{H}), 7.50(\mathrm{~d}, J=6.0 \mathrm{~Hz}, 2 \mathrm{H}), 7.44(\mathrm{~d}, J=6.0 \mathrm{~Hz}, 2 \mathrm{H}), 7.36-7.21(\mathrm{~m}, 6 \mathrm{H}), 5.44(\mathrm{~s}, 2 \mathrm{H}), 5.17(\mathrm{~s}, 2 \mathrm{H})$, 4.47 (sep, $J=6.0 \mathrm{~Hz}, 1 \mathrm{H}), 2.49(\mathrm{~s}, 3 \mathrm{H}), 1.27(\mathrm{~d}, J=6.0 \mathrm{~Hz}, 6 \mathrm{H}) \mathrm{ppm} .{ }^{13} \mathrm{C} \mathrm{NMR}\left(126 \mathrm{MHz}, \mathrm{CDCl}_{3}\right): \delta 171.4$ (C), 162.6 (C), 160.6 (C), 156.9 (C), 151.2 (C), 137.1 (C), 136.7 (C), $128.9(\mathrm{CH}), 128.5(\mathrm{CH}), 128.5(\mathrm{CH})$, $128.3(\mathrm{CH}), 127.6(\mathrm{CH}), 127.5(\mathrm{CH}), 87.7(\mathrm{C}), 45.2\left(\mathrm{CH}_{2}\right), 44.3\left(\mathrm{CH}_{2}\right), 42.5(\mathrm{CH}), 26.9\left(\mathrm{CH}_{3}\right), 22.7\left(\mathrm{CH}_{3}\right)$ ppm. HRMS (ESI): $m / z$ calculated for $\mathrm{C}_{24} \mathrm{H}_{26} \mathrm{~N}_{5} \mathrm{O}_{2}[\mathrm{M}+\mathrm{H}]^{+}=416.2081$, found $=416.2077$. M.p.: $121^{\circ} \mathrm{C}$. 
<smiles>Cc1nc(NC2CCCCC2)c2c(=O)n(Cc3ccccc3)c(=O)n(Cc3ccccc3)c2n1</smiles>

The general procedure $\mathrm{C}$ was applied using $N$-(1,3-dibenzyl-2,6-dioxo-1,2,3,6tetrahydropyrimidin-4-yl)acetimidamide (1e, $174 \mathrm{mg}, 0.5 \mathrm{mmol}, 1.0$ equiv) and cyclohexyl isocyanide ( $124 \mu \mathrm{L}, 1.0 \mathrm{mmol}, 2.0$ equiv). The compound was purified by manual flash chromatography using cHex:EtOAc (30:1) as eluent. The title compound was isolated as a white solid (153 mg, 67\%). Unknown compound according to a Sci-finder search.

$\mathbf{R}_{\mathbf{F}}=0.25$ (EtOAc: $\left.\mathrm{cHex}_{\mathrm{NEt}}=1: 20: 1\right) .{ }^{1} \mathrm{H}$ NMR $\left(300 \mathrm{MHz}, \mathrm{CDCl}_{3}\right): \delta 8.96(\mathrm{~d}, J=$ $9 \mathrm{~Hz}, 1 \mathrm{H}), 7.53(\mathrm{~d}, J=6.0 \mathrm{~Hz}, 2 \mathrm{H}), 7.48(\mathrm{~d}, J=6.0 \mathrm{~Hz}, 2 \mathrm{H}), 7.38-7.24(\mathrm{~m}, 6 \mathrm{H})$, $5.47(\mathrm{~s}, 2 \mathrm{H}), 5.20(\mathrm{~s}, 2 \mathrm{H}), 4.28-4.14(\mathrm{~m}, 1 \mathrm{H}), 2.52(\mathrm{~s}, 3 \mathrm{H}), 2.07-1.96(\mathrm{~m}, 2 \mathrm{H}), 1.85-1.75(\mathrm{~m}, 2 \mathrm{H}), 1.72$ - $1.61(\mathrm{~m}, 1 \mathrm{H}), 1.55-1.21(\mathrm{~m}, 5 \mathrm{H}) \mathrm{ppm} .{ }^{13} \mathrm{C} \mathrm{NMR}\left(126 \mathrm{MHz}, \mathrm{CDCl}_{3}\right): \delta 171.4(\mathrm{C}), 162.6(\mathrm{C}), 160.5(\mathrm{C})$, $156.9(\mathrm{C}), 151.2(\mathrm{C}), 137.1(\mathrm{C}), 136.7(\mathrm{C}), 128.9(\mathrm{CH}), 128.5(\mathrm{CH}), 128.4(\mathrm{CH}), 128.3(\mathrm{CH}), 127.6(\mathrm{CH})$, $127.5(\mathrm{CH}), 87.7(\mathrm{C}), 49.1(\mathrm{CH}), 45.1\left(\mathrm{CH}_{2}\right), 44.3\left(\mathrm{CH}_{2}\right), 32.8\left(\mathrm{CH}_{2}\right), 26.9\left(\mathrm{CH}_{3}\right), 25.6\left(\mathrm{CH}_{2}\right), 24.6\left(\mathrm{CH}_{2}\right)$ ppm. HRMS (ESI): $\mathrm{m} / z$ calculated for $\mathrm{C}_{27} \mathrm{H}_{30} \mathrm{~N}_{5} \mathrm{O}_{2}[\mathrm{M}+\mathrm{H}]^{+}=456.2394$, found $=456.2394$. M.p.: $127^{\circ} \mathrm{C}$.

\section{1,3-dibenzyl-7-methyl-5-(pentylamino)pyrimido[4,5-d]pyrimidine-2,4(1H,3H)-dione (5d) [HL129]}<smiles>CCCCCNc1nc(C)nc2c1c(=O)n(Cc1ccccc1)c(=O)n2Cc1ccccc1</smiles>
The general procedure $C$ was applied using $N$-(1,3-dibenzyl-2,6-dioxo1,2,3,6-tetrahydropyrimidin-4-yl)acetimidamide (1e, $174 \mathrm{mg}, 0.5 \mathrm{mmol}$, 1.0 equiv) and $n$-pentyl isocyanide (126 $\mu \mathrm{L}, 1.0 \mathrm{mmol}, 2.0$ equiv). The compound was purified by manual flash chromatography using cHex:EtOAc (10:1) as eluent. The title compound was isolated as a yellow oil (126 mg, 57\%). Unknown compound according to a Sci-finder search.

$\mathbf{R}_{\mathbf{F}}=0.21$ (cHex : EtOAc: $\left.\mathrm{NEt}_{3}=10: 1: 1\right) .{ }^{1} \mathrm{H}$ NMR $\left(300 \mathrm{MHz}, \mathrm{CDCl}_{3}\right): \delta 9.05-8.85(\mathrm{~m}, 1 \mathrm{H}), 7.50(\mathrm{~d}, J=6.0$ $\mathrm{Hz}, 2 \mathrm{H}), 7.44(\mathrm{~d}, J=6.0 \mathrm{~Hz}, 2 \mathrm{H}), 7.36-7.20(\mathrm{~m}, 6 \mathrm{H}), 5.44(\mathrm{~s}, 2 \mathrm{H}), 5.17(\mathrm{~s}, 2 \mathrm{H}), 3.55(\mathrm{q}, J=6.0 \mathrm{~Hz}, 2 \mathrm{H})$, $2.49(\mathrm{~s}, 3 \mathrm{H}), 1.73-1.50(\mathrm{~m}, 2 \mathrm{H}), 1.45-1.30(\mathrm{~m}, 4 \mathrm{H}), 1.00-0.80(\mathrm{~m}, 3 \mathrm{H}) \mathrm{ppm} .{ }^{13} \mathrm{C}$ NMR $\left(126 \mathrm{MHz}, \mathrm{CDCl}_{3}\right)$ : $\delta 171.4(\mathrm{C}), 162.7$ (C), 161.4 (C), 156.9 (C), 151.2 (C), 137.0 (C), 136.7 (C), 128.9 (CH), $128.6(\mathrm{CH}), 128.5$ $(\mathrm{CH}), 128.3(\mathrm{CH}), 127.6(\mathrm{CH}), 127.6(\mathrm{CH}), 87.9(\mathrm{C}), 45.2(\mathrm{CH}), 44.4\left(\mathrm{CH}_{2}\right), 40.8\left(\mathrm{CH}_{2}\right), 29.7\left(\mathrm{CH}_{2}\right), 29.1$ $\left(\mathrm{CH}_{2}\right), 26.9\left(\mathrm{CH}_{3}\right), 22.3\left(\mathrm{CH}_{2}\right), 14.0\left(\mathrm{CH}_{3}\right)$ ppm. HRMS (ESI): $\mathrm{m} / z$ calculated for $\mathrm{C}_{26} \mathrm{H}_{30} \mathrm{~N}_{5} \mathrm{O}_{2}[\mathrm{M}+\mathrm{H}]^{+}=$ 444.2394 , found $=444.2394$.

\section{1,3-dibenzyl-5-(benzylamino)-7-methylpyrimido[4,5-d]pyrimidine-2,4(1H,3H)-dione (5e) [HL130]}<smiles>Cc1nc(NCc2ccccc2)c2c(=O)n(Cc3ccccc3)c(=O)n(Cc3ccccc3)c2n1</smiles>

The general procedure $C$ was applied using $N$-(1,3-dibenzyl-2,6-dioxo1,2,3,6-tetrahydropyrimidin-4-yl)acetimidamide (1e, $174 \mathrm{mg}, 0.5 \mathrm{mmol}, 1.0$ equiv) and benzyl isocyanide ( $122 \mu \mathrm{L}, 1.0 \mathrm{mmol}, 2.0$ equiv). The compound was purified by manual flash chromatography using cHex:EtOAc (10:1) as eluent. The title compound was isolated as a green oil (40 mg, 17\%). Unknown compound according to a Sci-finder search.

$\mathbf{R}_{\mathbf{F}}=0.18$ (cHex : EtOAc: $\left.\mathrm{NEt}_{3}=10: 1: 0.01\right) .{ }^{1} \mathbf{H} \mathbf{N M R}\left(300 \mathrm{MHz}, \mathrm{CDCl}_{3}\right): \delta 9.27(\mathrm{~s}, 1 \mathrm{H}), 7.50(\mathrm{~d}, J=6.0$ $\mathrm{Hz}, 2 \mathrm{H}), 7.43(\mathrm{~d}, J=6.0 \mathrm{~Hz}, 2 \mathrm{H}), 7.38-7.31(\mathrm{~m}, 5 \mathrm{H}), 7.30-7.20(\mathrm{~m}, 6 \mathrm{H}), 5.45(\mathrm{~s}, 2 \mathrm{H}), 5.16(\mathrm{~s}, 2 \mathrm{H}), 4.86-$ $4.74(\mathrm{~m}, 2 \mathrm{H}), 2.52(\mathrm{~s}, 3 \mathrm{H}) \mathrm{ppm} .{ }^{13} \mathrm{C} \mathrm{NMR}\left(126 \mathrm{MHz}, \mathrm{CDCl}_{3}\right): \delta 171.5(\mathrm{C}), 162.6$ (C), 161.4 (C), 156.9 (C), $151.1(\mathrm{C}), 138.1(\mathrm{C}), 136.9(\mathrm{C}), 136.6(\mathrm{C}), 128.9(\mathrm{CH}), 128.6(\mathrm{CH}), 128.6(\mathrm{CH}), 128.5(\mathrm{CH}), 128.3(\mathrm{CH})$, $127.8(\mathrm{CH}), 127.6(\mathrm{CH}), 127.6(\mathrm{CH}), 127.4(\mathrm{CH}), 88.1(\mathrm{C}), 45.3\left(\mathrm{CH}_{2}\right), 44.6\left(\mathrm{CH}_{2}\right), 44.4\left(\mathrm{CH}_{2}\right), 26.9\left(\mathrm{CH}_{3}\right)$ ppm. HRMS (ESI): $m / z$ calculated for $\mathrm{C}_{28} \mathrm{H}_{26} \mathrm{~N}_{5} \mathrm{O}_{2}[\mathrm{M}+\mathrm{H}]^{+}=464.2081$, found $=464.2068$.

\section{1,3-dibenzyl-7-methyl-5-((2,4,4-trimethylpentan-2-yl)amino)pyrimido[4,5-d]pyrimidine-2,4(1H,3H)- dione (5f) [HL132]}

The general procedure $\mathrm{C}$ was applied using $\mathrm{N}$-(1,3-dibenzyl-2,6-dioxo-1,2,3,6-tetrahydropyrimidin-4$\mathrm{yl}$ )acetimidamide (1e, $174 \mathrm{mg}, 0.5 \mathrm{mmol}, 1.0$ equiv) and 2-isocyano-2,4,4-trimethylpentane (175 $\mu \mathrm{L}$, $1.0 \mathrm{mmol}, 2.0$ equiv). The compound was purified by manual flash chromatography using cHex:EtOAc 
<smiles>Cc1nc(NC(C)(C)CC(C)(C)C)c2c(=O)n(Cc3ccccc3)c(=O)n(Cc3ccccc3)c2n1</smiles>

(30:1) as eluent. The title compound was isolated as a green oil (188 mg, $77 \%)$. Unknown compound according to a Sci-finder search.

$\mathbf{R}_{\mathrm{F}}=0.53$ (cHex : EtOAc: $\left.\mathrm{NEt}_{3}=10: 1: 1\right) .{ }^{1} \mathrm{H} \mathrm{NMR}\left(300 \mathrm{MHz}, \mathrm{CDCl}_{3}\right): \delta 9.15$ $(\mathrm{s}, 1 \mathrm{H}), 7.53(\mathrm{~d}, J=6.0 \mathrm{~Hz}, 2 \mathrm{H}), 7.46(\mathrm{~d}, J=6.0 \mathrm{~Hz}, 2 \mathrm{H}), 7.37-7.23(\mathrm{~m}, 6 \mathrm{H})$, $5.44(\mathrm{~s}, 2 \mathrm{H}), 5.19(\mathrm{~s}, 2 \mathrm{H}), 2.50(\mathrm{~s}, 3 \mathrm{H}), 1.99(\mathrm{~s}, 2 \mathrm{H}), 1.58(\mathrm{~s}, 6 \mathrm{H}), 1.00(\mathrm{~s}, 9 \mathrm{H})$ ppm. ${ }^{13} \mathrm{C} \mathrm{NMR}\left(150.90 \mathrm{MHz}, \mathrm{CDCl}_{3}\right): \delta 170.2$ (C), 162.8 (C), 161.0 (C), 156.9 (C), $151.1(\mathrm{C}), 137.1(\mathrm{C}), 136.7(\mathrm{C}), 129.0(\mathrm{CH}), 128.6(\mathrm{CH}), 128.4(\mathrm{CH}), 128.3$ $(\mathrm{CH}), 127.5(\mathrm{CH}), 127.5(\mathrm{CH}), 88.0(\mathrm{C}), 56.4(\mathrm{C}), 51.2\left(\mathrm{CH}_{2}\right), 45.0\left(\mathrm{CH}_{2}\right), 44.2\left(\mathrm{CH}_{2}\right), 31.7(\mathrm{C}), 31.5\left(\mathrm{CH}_{3}\right)$, $29.5\left(\mathrm{CH}_{3}\right), 27.0\left(\mathrm{CH}_{3}\right)$ ppm. HRMS (ESI): $\mathrm{m} / z$ calculated for $\mathrm{C}_{29} \mathrm{H}_{36} \mathrm{~N}_{5} \mathrm{O}_{2}[\mathrm{M}+\mathrm{H}]^{+}=486.2839$, found $=$ 486.2864.

\section{1,3-dibenzyl-7-methyl-5-((2-morpholinoethyl)amino)pyrimido[4,5- $d$ ]pyrimidine-2,4(1H,3H)-dione} (5g) [HL135]<smiles>Cc1nc(NCCN2CCOCC2)c2c(=O)n(Cc3ccccc3)c(=O)n(Cc3ccccc3)c2n1</smiles>

The general procedure $C$ was applied using $N$-(1,3-dibenzyl-2,6-dioxo1,2,3,6-tetrahydropyrimidin-4-yl)acetimidamide (1e, $174 \mathrm{mg}, 0.5 \mathrm{mmol}$, 1.0 equiv) and 2-morpholinoethyl isocyanide ( $140 \mu \mathrm{L}, 1.0 \mathrm{mmol}, 2.0$ equiv). The compound was purified by manual flash chromatography using cHex:EtOAc (gradient $=6: 1-3: 1$ ) as eluent. The title compound was isolated as a green oil (60 mg, 25\%). Unknown compound according to a Sci-finder search.

$\mathbf{R}_{\mathrm{F}}=0.32$ (EtOAC: DCM = 2:3). ${ }^{1} \mathbf{H}$ NMR $\left(300 \mathrm{MHz}, \mathrm{CDCl}_{3}\right): \delta 9.25-9.10$ (m, 1H), $7.54-7.42(\mathrm{~m}, 4 \mathrm{H}), 7.35-7.21(\mathrm{~m}, 6 \mathrm{H}), 5.43(\mathrm{~s}, 2 \mathrm{H}), 5.18(\mathrm{~s}, 2 \mathrm{H}), 3.80-3.73(\mathrm{~m}, 4 \mathrm{H}), 3.72-3.64$ $(\mathrm{m}, 2 \mathrm{H}), 2.68-2.58(\mathrm{~m}, 2 \mathrm{H}), 2.57-2.50(\mathrm{~m}, 4 \mathrm{H}), 2.48(\mathrm{~s}, 3 \mathrm{H}) \mathrm{ppm} .{ }^{13} \mathrm{C} \mathrm{NMR}\left(126 \mathrm{MHz}, \mathrm{CDCl}_{3}\right): \delta 171.5(\mathrm{C})$, $162.5(\mathrm{C}), 161.5(\mathrm{C}), 157.0(\mathrm{C}), 151.3(\mathrm{C}), 137.1(\mathrm{C}), 136.8(\mathrm{C}), 129.0(\mathrm{CH}), 128.8(\mathrm{CH}), 128.5(\mathrm{CH}), 128.4$ $(\mathrm{CH}), 127.7(\mathrm{CH}), 127.7(\mathrm{CH}), 88.3(\mathrm{C}), 67.0\left(\mathrm{CH}_{2}\right), 57.1\left(\mathrm{CH}_{2}\right), 53.5\left(\mathrm{CH}_{2}\right), 45.3\left(\mathrm{CH}_{2}\right), 44.4\left(\mathrm{CH}_{2}\right), 37.5$ $\left(\mathrm{CH}_{2}\right), 27.0\left(\mathrm{CH}_{3}\right)$ ppm. HRMS (ESI): $\mathrm{m} / z$ calculated for $\mathrm{C}_{27} \mathrm{H}_{31} \mathrm{~N}_{6} \mathrm{O}_{3}[\mathrm{M}+\mathrm{H}]^{+}=487.2434$, found $=$ 487.2452 .

\section{1,3-dibenzyl-5-((2,6-dimethylphenyl)amino)-7-methylpyrimido[4,5-d]pyrimidine-2,4(1H,3H)-dione} (5h) [HL114]<smiles>Cc1nc(Nc2c(C)cccc2C)c2c(=O)n(Cc3ccccc3)c(=O)n(Cc3ccccc3)c2n1</smiles>

The general procedure $C$ was applied using $N$-(1,3-dibenzyl-2,6-dioxo-1,2,3,6tetrahydropyrimidin-4-yl)acetimidamide (1e, $174 \mathrm{mg}, 0.5 \mathrm{mmol}, 1.0$ equiv) and 2,6-dimethylphenylisocyanide (131 $\mathrm{mg}, 1.0 \mathrm{mmol}, 2.0$ equiv). The compound was purified by manual flash chromatography using cHex:EtOAc $(20: 1)$ as eluent. The title compound was isolated as an off-white solid (184 $\mathrm{mg}, 77 \%)$. Unknown compound according to a Sci-finder search.

$\mathbf{R}_{\mathbf{F}}=0.13$ (cHex : EtOAc: $\left.\mathrm{NEt}_{3}=20: 1: 0.01\right) .{ }^{1} \mathbf{H} \mathbf{~ N M R}\left(300 \mathrm{MHz}, \mathrm{CDCl}_{3}\right): \delta 10.30$ (bs, $1 \mathrm{H}), 7.55(\mathrm{~d}, J=9.0 \mathrm{~Hz}, 2 \mathrm{H}), 7.51(\mathrm{~d}, J=9.0 \mathrm{~Hz}, 2 \mathrm{H}), 7.38-7.23(\mathrm{~m}, 6 \mathrm{H})$, $7.20-2.05(\mathrm{~m}, 3 \mathrm{H}), 5.46(\mathrm{~s}, 2 \mathrm{H}), 5.23(\mathrm{~s}, 2 \mathrm{H}), 2.40(\mathrm{~s}, 3 \mathrm{H}), 2.20(\mathrm{~s}, 6 \mathrm{H}) \mathrm{ppm} .{ }^{13} \mathrm{C}$ NMR $\left(126 \mathrm{MHz}, \mathrm{CDCl}_{3}\right)$ : $\delta 171.9$ (C), 163.0 (C), 160.7 (C), 157.0 (C), 151.2 (C), 136.9 (C), 136.6 (C), 135.5 (C), 134.7 (C), 129.1 $(\mathrm{CH}), 128.9(\mathrm{CH}), 128.5(\mathrm{CH}), 128.4(\mathrm{CH}), 128.0(\mathrm{CH}), 127.7(\mathrm{CH}), 127.7(\mathrm{CH}), 127.1(\mathrm{CH}), 88.2(\mathrm{C}), 45.3$ $\left(\mathrm{CH}_{2}\right), 44.6\left(\mathrm{CH}_{2}\right), 27.0\left(\mathrm{CH}_{3}\right), 18.8\left(\mathrm{CH}_{3}\right)$ ppm. HRMS (ESI): $\mathrm{m} / z$ calculated for $\mathrm{C}_{29} \mathrm{H}_{28} \mathrm{~N}_{5} \mathrm{O}_{2}[\mathrm{M}+\mathrm{H}]^{+}=$ 478.2230, found $=478.2238$. M.p.: $179^{\circ} \mathrm{C}$.

\section{1,3-dibenzyl-5-(tert-butylamino)-7-isopropylpyrimido[4,5-d]pyrimidine-2,4(1H,3H)-dione}<smiles>CC(C)c1nc(NC(C)(C)C)c2c(=O)n(Cc3ccccc3)c(=O)n(Cc3ccccc3)c2n1</smiles>
[HL111]

The general procedure $C$ was applied using $N$-(1,3-dibenzyl-2,6-dioxo-1,2,3,6tetrahydropyrimidin-4-yl)isobutyrimidamide (1f, $188 \mathrm{mg}, 0.5 \mathrm{mmol}, 1.0$ equiv) and tert-butyl isocyanide (2a, $131 \mathrm{mg}, 1.0 \mathrm{mmol}, 2.0$ equiv). The compound was purified by manual flash chromatography using heptane / EtOAc () as eluent. The title compound was isolated as a yellow oil (225 mg, 98\%). Unknown compound according to a Sci-finder search. 


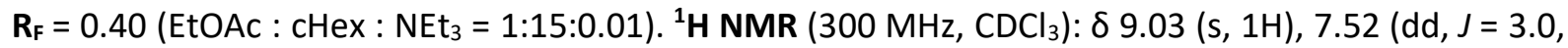
$9.0 \mathrm{~Hz}, 2 \mathrm{H}), 7.43(\mathrm{dd}, J=3.0,9.0 \mathrm{~Hz}, 2 \mathrm{H}), 7.35-7.20(\mathrm{~m}, 6 \mathrm{H}), 5.45(\mathrm{~s}, 2 \mathrm{H}), 5.17(\mathrm{~s}, 2 \mathrm{H}), 2.96$ (hep, $J=6.0$ $\mathrm{Hz}, 1 \mathrm{H}), 1.52(\mathrm{~s}, 9 \mathrm{H}), 1.29(\mathrm{~d}, 9.0 \mathrm{~Hz}, 6 \mathrm{H}) \mathrm{ppm} .{ }^{13} \mathrm{C}$ NMR (126 MHz, CDCl $): \delta 177.5$ (C), $162.8(\mathrm{C}), 161.3$ (C), $156.9(\mathrm{C}), 151.3(\mathrm{C}), 137.2(\mathrm{C}), 136.7(\mathrm{C}), 129.0(\mathrm{CH}), 128.4(\mathrm{CH}), 128.4(\mathrm{CH}), 128.3(\mathrm{CH}), 127.5(\mathrm{CH})$, $127.5(\mathrm{CH}), 88.3(\mathrm{C}), 52.5(\mathrm{C}), 45.2\left(\mathrm{CH}_{2}\right), 44.3\left(\mathrm{CH}_{2}\right), 38.1(\mathrm{CH}), 29.0\left(\mathrm{CH}_{3}\right), 21.2\left(\mathrm{CH}_{3}\right)$ ppm. HRMS (ESI): $m / z$ calculated for $\mathrm{C}_{27} \mathrm{H}_{32} \mathrm{~N}_{5} \mathrm{O}_{2}[\mathrm{M}+\mathrm{H}]^{+}=458.2551$, found $=458.2551$.

1,3-dibenzyl-5-(cyclohexylamino)-7-isopropylpyrimido[4,5-d]pyrimidine-2,4(1H,3H)-dione [JC901]<smiles>CC(C)c1nc(NC2CCCCC2)c2c(=O)n(Cc3ccccc3)c(=O)n(Cc3ccccc3)c2n1</smiles>

The general procedure $C$ was applied using $N$-(1,3-dibenzyl-2,6-dioxo-1,2,3,6tetrahydropyrimidin-4-yl)isobutyrimidamide (1f, $188 \mathrm{mg}, 0.5 \mathrm{mmol}, 1.0$ equiv) and cyclohexyl isocyanide ( $124 \mu \mathrm{L}, 1.0 \mathrm{mmol}, 2.0$ equiv). The compound was purified by manual flash chromatography using heptane / EtOAc () as eluent. The title compound was isolated as a white solid (200 mg, 83\%). Unknown compound according to a Sci-finder search.

$\mathbf{R}_{\mathbf{F}}=0.19$ (cHex : EtOAc: $\left.\mathrm{NEt}_{3} ; 9: 1: 0.01\right) .{ }^{1}{ }^{\mathbf{H}} \mathbf{N M R}\left(300 \mathrm{MHz}, \mathrm{CDCl}_{3}\right): \delta 8.88$ $(\mathrm{d}, J=7.8 \mathrm{~Hz}, 1 \mathrm{H}), 7.59-7.52(\mathrm{~m}, 2 \mathrm{H}), 7.49-7.44(\mathrm{~m}, 2 \mathrm{H}), 7.37-7.24(\mathrm{~m}, 6 \mathrm{H})$, $5.48(\mathrm{~s}, 2 \mathrm{H}), 5.21(\mathrm{~s}, 2 \mathrm{H}), 4.29-4.07(\mathrm{~m}, 1 \mathrm{H}), 2.97$ (hept, $J=6.9 \mathrm{~Hz}, 1 \mathrm{H}), 2.04(\mathrm{~m}, 2 \mathrm{H}), 1.79(\mathrm{~m}, 2 \mathrm{H}), 1.73$ - $1.61(\mathrm{~m}, 1 \mathrm{H}), 1.40(\mathrm{~m}, 5 \mathrm{H}), 1.31(\mathrm{~d}, J=6.8 \mathrm{~Hz}, 6 \mathrm{H}) \mathrm{ppm} .{ }^{13} \mathrm{C} \mathrm{NMR}\left(126 \mathrm{MHz}, \mathrm{CDCl}_{3}\right): \delta 178.4(\mathrm{C}), 162.7$ (C), $160.8(\mathrm{C}), 157.1(\mathrm{C}), 151.4(\mathrm{C}), 137.3(\mathrm{C}), 136.8(\mathrm{C}), 129.0(\mathrm{CH}), 128.5(\mathrm{CH}), 128.4(\mathrm{CH}), 128.2(\mathrm{CH})$, $127.5(\mathrm{CH}), 127.5(\mathrm{CH}), 88.0(\mathrm{C}), 49.3(\mathrm{CH}), 45.2\left(\mathrm{CH}_{2}\right), 44.3\left(\mathrm{CH}_{2}\right), 38.1(\mathrm{CH}), 32.6\left(\mathrm{CH}_{2}\right), 25.7\left(\mathrm{CH}_{2}\right), 24.7$ $\left(\mathrm{CH}_{2}\right), 21.2\left(\mathrm{CH}_{3}\right)$ ppm. HRMS (ESI): $\mathrm{m} / z$ calculated for $\mathrm{C}_{29} \mathrm{H}_{34} \mathrm{~N}_{5} \mathrm{O}_{2}[\mathrm{M}+\mathrm{H}]^{+}=484.2707$, found $=$ 484.2701. M.p.: $91^{\circ} \mathrm{C}$.

\section{1,3-dibenzyl-5-((2,6-dimethylphenyl)amino)-7-isopropylpyrimido[4,5-d]pyrimidine-2,4(1H,3H)- dione $(5 \mathrm{~m})$ [JC901B]}<smiles>Cc1cccc(C)c1Nc1nc(C(C)C)nc2c1c(=O)n(Cc1ccccc1)c(=O)n2Cc1ccccc1</smiles>

The general procedure $\mathrm{C}$ was applied using $N$-(1,3-dibenzyl-2,6-dioxo-1,2,3,6tetrahydropyrimidin-4-yl)isobutyrimidamide (1f, $188 \mathrm{mg}, 0.5 \mathrm{mmol}, 1.0$ equiv) and 2,6-dimethylphenylisocyanide (131 $\mathrm{mg}, 1.0 \mathrm{mmol}, 2.0$ equiv). The compound was purified by manual flash chromatography using cHex:EtOAc:NEt ${ }_{3}$ (10:1:0.01) as eluent. The title compound was isolated as a white solid (252 mg, 99\%). Unknown compound according to a Sci-finder search.

$\mathbf{R}_{\mathbf{F}}=0.25$ (cHex : EtOAc: $\left.\mathrm{NEt}_{3} ; 9: 1: 0.01\right) .{ }^{1} \mathbf{H} \mathbf{~ N M R}\left(300 \mathrm{MHz}, \mathrm{CDCl}_{3}\right): \delta 10.26$ (bs, $1 \mathrm{H}), 7.61(\mathrm{~d}, J=6.6 \mathrm{~Hz}, 2 \mathrm{H}), 7.53(\mathrm{~d}, J=7.0 \mathrm{~Hz}, 2 \mathrm{H}), 7.40-7.25(\mathrm{~m}, 6 \mathrm{H}), 7.19-7.02(\mathrm{~m}, 3 \mathrm{H}), 5.50(\mathrm{~s}$, $2 \mathrm{H}), 5.26(\mathrm{~s}, 2 \mathrm{H}), 2.86$ (hept, $J=6.9 \mathrm{~Hz}, 1 \mathrm{H}), 2.22(\mathrm{~s}, 6 \mathrm{H}), 1.18(\mathrm{~d}, J=6.8 \mathrm{~Hz}, 6 \mathrm{H}) \mathrm{ppm} .{ }^{13} \mathrm{C}$ NMR $(125$ $\mathrm{MHz}, \mathrm{CDCl}_{3}$ ) $\delta 178.9$ (C), 163.0 (C), 160.9 (C), 157.2 (C), 151.3 (C), 137.1 (C), 136.7 (C), 135.5 (C), 134.9 (C), $129.3(\mathrm{CH}), 128.8(\mathrm{CH}), 128.5(\mathrm{CH}), 128.3(\mathrm{CH}), 127.9(\mathrm{CH}), 127.8(\mathrm{CH}), 127.7(\mathrm{CH}), 126.9(\mathrm{CH}), 88.5$ (C), $45.4\left(\mathrm{CH}_{2}\right), 44.6\left(\mathrm{CH}_{2}\right), 37.9(\mathrm{CH}), 21.0\left(\mathrm{CH}_{3}\right), 18.7\left(\mathrm{CH}_{3}\right)$ ppm. HRMS (ESI): $\mathrm{m} / z$ calculated for $\mathrm{C}_{31} \mathrm{H}_{32} \mathrm{~N}_{5} \mathrm{O}_{2}[\mathrm{M}+\mathrm{H}]^{+}=506.2551$, found $=506.2537$. M.p.: $83-84^{\circ} \mathrm{C}$.

\section{1,3-dibenzyl-7-(tert-butyl)-5-(tert-butylamino)pyrimido[4,5-d]pyrimidine-2,4(1H,3H)-dione} [HLO70]<smiles>CC(C)(C)Nc1nc(C(C)(C)C)nc2c1c(=O)n(Cc1ccccc1)c(=O)n2Cc1ccccc1</smiles>

The general procedure $C$ was applied using $N^{\prime}$-(1,3-dibenzyl-2,6-dioxo-1,2,3,6tetrahydropyrimidin-4-yl)pivalimidamide (1g, $195 \mathrm{mg}, 0.5 \mathrm{mmol}, 1.0$ equiv) and tert-butyl isocyanide (2a, $119 \mu \mathrm{L}, 1.0 \mathrm{mmol}, 2.0$ equiv). The compound was purified by manual flash chromatography using cHex:EtOAc $(15: 1)$ as eluent. The title compound was isolated as a colorless oil (224 mg, 95\%). Unknown compound according to a Sci-finder search.

$\mathbf{R}_{\mathrm{F}}=0.33$ (cHex : EtOAc: NEt $\left.{ }_{3}=20: 1: 0.01\right) .{ }^{1} \mathbf{H}$ NMR $\left(300 \mathrm{MHz}, \mathrm{CDCl}_{3}\right): \delta 9.20-$ $9.12(\mathrm{~m}, 1 \mathrm{H}), 7.67-7.59(\mathrm{~m}, 2 \mathrm{H}), 7.57-7.50(\mathrm{~m}, 2 \mathrm{H}), 7.42-7.34(\mathrm{~m}, 4 \mathrm{H}), 7.33-7.28(\mathrm{~m}, 2 \mathrm{H}), 5.54(\mathrm{~s}$, 2H), $5.27(\mathrm{~s}, 2 \mathrm{H}), 1.70-1.55(\mathrm{~m}, 9 \mathrm{H}), 1.50-1.40(\mathrm{~m}, 9 \mathrm{H}) \mathrm{ppm} .{ }^{13} \mathrm{C} \mathrm{NMR}\left(126 \mathrm{MHz}, \mathrm{CDCl}_{3}\right): \delta 179.3(\mathrm{C})$, 
162.7 (C), 161.0 (C), 156.8 (C), 151.2 (C), 137.2 (C), 136.7 (C), 128.8 (CH), $128.4(\mathrm{CH}), 128.4(\mathrm{CH}), 128.3$ $(\mathrm{CH}), 128.2(\mathrm{CH}), 127.4(\mathrm{CH}), 88.0(\mathrm{C}), 52.2(\mathrm{C}), 45.2\left(\mathrm{CH}_{2}\right), 44.2\left(\mathrm{CH}_{2}\right), 40.1(\mathrm{C}), 29.1\left(\mathrm{CH}_{3}\right), 28.9\left(\mathrm{CH}_{3}\right)$ ppm. HRMS (ESI): $m / z$ calculated for $\mathrm{C}_{28} \mathrm{H}_{34} \mathrm{~N}_{5} \mathrm{O}_{2}[\mathrm{M}+\mathrm{H}]^{+}=472.2704$, found $=472.2707$.

\section{1,3-dibenzyl-7-(tert-butyl)-5-(cyclohexylamino)pyrimido[4,5-d]pyrimidine-2,4(1H,3H)-dione [HL073]}<smiles>CC(C)(C)c1nc(NC2CCCCC2)c2c(=O)n(Cc3ccccc3)c(=O)n(Cc3ccccc3)c2n1</smiles>

The general procedure $C$ was applied using $N^{\prime}$-(1,3-dibenzyl-2,6-dioxo-1,2,3,6tetrahydropyrimidin-4-yl)pivalimidamide $(\mathbf{1 g}, 195 \mathrm{mg}, 0.5 \mathrm{mmol}, 1.0$ equiv) and cyclohexyl isocyanide ( $124 \mu \mathrm{L}, 1.0 \mathrm{mmol}, 2.0$ equiv). The compound was purified by manual flash chromatography using cHex:EtOAc:NEt 3 (20:1:0.01) as eluent. The title compound was isolated as a white solid (194 mg, 81\%). Unknown compound according to a Sci-finder search.

$\mathbf{R}_{\mathbf{F}}=0.20$ (EtOAc: $\left.\mathrm{cHex}_{\mathrm{NEt}}=1: 20: 0.01\right) .{ }^{1} \mathbf{H} \mathbf{~ N M R}\left(500 \mathrm{MHz}, \mathrm{CDCl}_{3}\right): \delta 8.84$ $(\mathrm{d}, J=3.0 \mathrm{~Hz}, 1 \mathrm{H}), 7.55(\mathrm{~d}, J=10.0 \mathrm{~Hz}, 2 \mathrm{H}), 7.47(\mathrm{~d}, J=10.0 \mathrm{~Hz}, 2 \mathrm{H}), 7.36-7.25$ $(\mathrm{m}, 6 \mathrm{H}), 5.49(\mathrm{~s}, 2 \mathrm{H}), 5.22(\mathrm{~s}, 2 \mathrm{H}), 4.20-4.12(\mathrm{~m}, 1 \mathrm{H}), 2.09-2.02(\mathrm{~m}, 2 \mathrm{H}), 1.84-1.76(\mathrm{~m}, 2 \mathrm{H}), 1.70-$ $1.63(\mathrm{~m}, 1 \mathrm{H}), 1.50-1.38(\mathrm{~m}, 4 \mathrm{H}), 1.37(\mathrm{~s}, 9 \mathrm{H}), 1.36-1.23(\mathrm{~m}, 1 \mathrm{H}) \mathrm{ppm} .{ }^{13} \mathrm{C} \mathrm{NMR}\left(126 \mathrm{MHz}, \mathrm{CDCl}_{3}\right): \delta$ 180.1 (C), 162.6 (C), 160.5 (C), 156.9 (C), 151.4 (C), 137.2 (C), 136.7 (C), 128.8 (CH), 128.4 (CH), 128.4 $(\mathrm{CH}), 128.2(\mathrm{CH}), 127.5(\mathrm{CH}), 127.4(\mathrm{CH}), 87.7(\mathrm{C}), 49.5(\mathrm{CH}), 45.3\left(\mathrm{CH}_{2}\right), 44.2\left(\mathrm{CH}_{2}\right), 40.0(\mathrm{C}), 32.5\left(\mathrm{CH}_{2}\right)$, $29.1\left(\mathrm{CH}_{3}\right), 25.7\left(\mathrm{CH}_{2}\right), 24.7\left(\mathrm{CH}_{2}\right)$ ppm. HRMS (ESI): $\mathrm{m} / z$ calculated for $\mathrm{C}_{30} \mathrm{H}_{36} \mathrm{~N}_{5} \mathrm{O}_{2}[\mathrm{M}+\mathrm{H}]^{+}=498.2853$, found $=498.2864$. M.p.: $123^{\circ} \mathrm{C}$.

\section{1,3-dibenzyl-5-(benzylamino)-7-(tert-butyl)pyrimido[4,5-d]pyrimidine-2,4(1H,3H)-dione} [HL147]<smiles>CC(C)(C)c1nc(NCc2ccccc2)c2c(=O)n(Cc3ccccc3)c(=O)n(Cc3ccccc3)c2n1</smiles>

The general procedure $C$ was applied using $N^{\prime}$-(1,3-dibenzyl-2,6-dioxo1,2,3,6-tetrahydropyrimidin-4-yl)pivalimidamide $(1 \mathrm{~g}, 195 \mathrm{mg}, 0.5 \mathrm{mmol}$, 1.0 equiv) and benzyl isocyanide (120 $\mu \mathrm{L}, 1.0 \mathrm{mmol}, 2.0$ equiv). The compound was purified by manual flash chromatography using cHex:EtOAc $(40 ; 1)$ as eluent. The title compound was isolated as a white solid (202 mg, 80\%). Unknown compound according to a Sci-finder search.

$\mathbf{R}_{\mathrm{F}}=0.26$ (cHex : EtOAc : NEt $\left.\mathrm{N}_{3}=20: 1: 0.01\right) .{ }^{1}{ }^{\mathrm{H}} \mathrm{NMR}\left(300 \mathrm{MHz}, \mathrm{CDCl}_{3}\right): \delta 9.30-9.22(\mathrm{~m}, 1 \mathrm{H}), 7.54(\mathrm{dd}, J=$ $3.0,9.0 \mathrm{~Hz}, 2 \mathrm{H}), 7.45(\mathrm{dd}, J=3.0,9.0 \mathrm{~Hz}, 2 \mathrm{H}), 7.41-7.22(\mathrm{~m}, 11 \mathrm{H}), 5.49(\mathrm{~s}, 2 \mathrm{H}), 5.20(\mathrm{~s}, 2 \mathrm{H}), 4.81(\mathrm{~d}, J=$ $6 \mathrm{~Hz}, 2 \mathrm{H}), 1.36(\mathrm{~s}, 9 \mathrm{H}) \mathrm{ppm} .{ }^{13} \mathrm{C} \mathrm{NMR}\left(126 \mathrm{MHz}, \mathrm{CDCl}_{3}\right): \delta 180.5$ (C), $162.6(\mathrm{C}), 161.4$ (C), 156.9 (C), 151.4 (C), $138.6(\mathrm{C}), 137.1(\mathrm{C}), 136.7(\mathrm{C}), 128.8(\mathrm{CH}), 128.5(\mathrm{CH}), 128.5(\mathrm{CH}), 128.4(\mathrm{CH}), 128.3(\mathrm{CH}), 127.8$ $(\mathrm{CH}), 127.6(\mathrm{CH}), 127.5(\mathrm{CH}), 127.3(\mathrm{CH}), 88.1(\mathrm{C}), 45.4\left(\mathrm{CH}_{2}\right), 44.5\left(\mathrm{CH}_{2}\right), 44.4\left(\mathrm{CH}_{2}\right), 40.2(\mathrm{C}), 29.1\left(\mathrm{CH}_{3}\right)$ ppm. HRMS (ESI): $m / z$ calculated for $\mathrm{C}_{31} \mathrm{H}_{32} \mathrm{~N}_{5} \mathrm{O}_{2}[\mathrm{M}+\mathrm{H}]^{+}=506.2525$, found $=506.2551$. M.p.: $133^{\circ} \mathrm{C}$.

\section{1,3-dibenzyl-7-(tert-butyl)-5-[(2,6-dimethylphenyl)amino]pyrimido[4,5- $d]$ pyrimidine-2,4(1H,3H)- dione (5r) [HL076]}<smiles>Cc1cccc(C)c1Nc1nc(C(C)(C)C)nc2c1c(=O)n(Cc1ccccc1)c(=O)n2Cc1ccccc1</smiles>

The general procedure $C$ was applied using $N^{\prime}$-(1,3-dibenzyl-2,6-dioxo-1,2,3,6tetrahydropyrimidin-4-yl)pivalimidamide $(\mathbf{1 g}, 195 \mathrm{mg}, 0.5 \mathrm{mmol}, 1.0$ equiv) and 2,6-dimethylphenylisocyanide (131 $\mathrm{mg}, 1.0 \mathrm{mmol}, 2.0$ equiv). The compound was purified by manual flash chromatography using cHex:EtOAc:NEt ${ }_{3}$ (20:1:0.01) as eluent. The title compound was isolated as a white solid (242 mg, 93\%). Unknown compound according to a Sci-finder search.

$\mathbf{R}_{\mathbf{F}}=0.43$ (cHex : EtOAc: $\left.\mathrm{NEt}_{3}=20: 1: 0.01\right) .{ }^{1} \mathbf{H}$ NMR $\left(500 \mathrm{MHz}, \mathrm{CDCl}_{3}\right): \delta 10.22$

(brs, $1 \mathrm{H}), 7.64(\mathrm{~d}, J=10 \mathrm{~Hz}, 2 \mathrm{H}), 7.56(\mathrm{~d}, J=10 \mathrm{~Hz}, 2 \mathrm{H}), 7.40-7.34(\mathrm{~m}, 4 \mathrm{H}), 7.33-7.29(\mathrm{~m}, 2 \mathrm{H}), 7.19-$ $7.12(\mathrm{~m}, 3 \mathrm{H}), 5.54(\mathrm{~s}, 2 \mathrm{H}), 5.29(\mathrm{~s}, 2 \mathrm{H}), 2.24(\mathrm{~s}, 6 \mathrm{H}), 1.23(\mathrm{~s}, 9 \mathrm{H}) \mathrm{ppm} .{ }^{13} \mathrm{C} \mathrm{NMR}\left(126 \mathrm{MHz}, \mathrm{CDCl}_{3}\right): \delta 180.6$ (C), 162.9 (C), 160.7 (C), 157.0 (C), 151.4 (C), 137.1 (C), 136.6 (C), 135.4 (C), 134.9 (C), 129.1 (CH), 128.8 $(\mathrm{CH}), 128.5(\mathrm{CH}), 128.3(\mathrm{CH}), 127.7(\mathrm{CH}), 127.6(\mathrm{CH}), 127.6(\mathrm{CH}), 126.8(\mathrm{CH}), 88.1(\mathrm{C}), 45.4\left(\mathrm{CH}_{2}\right), 44.5$ $\left(\mathrm{CH}_{2}\right), 40.0$ (C), $28.9\left(\mathrm{CH}_{3}\right), 18.7\left(\mathrm{CH}_{3}\right)$ ppm. HRMS (ESI): $\mathrm{m} / z$ calculated for $\mathrm{C}_{32} \mathrm{H}_{34} \mathrm{~N}_{5} \mathrm{O}_{2}[\mathrm{M}+\mathrm{H}]^{+}=$ 520.2691, found $=520.2707$. M.p.: $154^{\circ} \mathrm{C}$. 
1,3-dibenzyl-7-(tert-butyl)-5-((4-methoxyphenyl)amino)pyrimido[4,5-d]pyrimidine-2,4(1H,3H)dione (5s) [HL148]<smiles>COc1ccc(Nc2nc(C(C)(C)C)nc3c2c(=O)n(Cc2ccccc2)c(=O)n3Cc2ccccc2)cc1</smiles>

The general procedure $C$ was applied using $N^{\prime}$-(1,3-dibenzyl-2,6-dioxo1,2,3,6-tetrahydropyrimidin-4-yl)pivalimidamide (1g, $205 \mathrm{mg}, 0.5 \mathrm{mmol}$, 1.0 equiv) and 4-methoxyphenyl isocyanide $(133 \mu \mathrm{g}, 1.0 \mathrm{mmol}, 2.0$ equiv). The compound was purified by manual flash chromatography using cHex:EtOAc (30:1) as eluent. The title compound was isolated as an off-white solid (170 mg, 65\%). Unknown compound according to a Sci-finder search.

$\mathbf{R}_{\mathbf{F}}=0.19$ (cHex : EtOAc: $\left.\mathrm{NEt}_{3}=20: 1: 0.01\right) .{ }^{1} \mathbf{H} \mathbf{~ N M R}\left(300 \mathrm{MHz}, \mathrm{CDCl}_{3}\right): \delta$ 10.95 (brs, 1H), 7.69 (d, $J=9.0 \mathrm{~Hz}, 2 \mathrm{H}$ ), 7.52 (dd, $J=3.0,9.0 \mathrm{~Hz}, 2 \mathrm{H}$ ), 7.47 (dd, $J=3.0,9.0 \mathrm{~Hz}, 2 \mathrm{H}$ ), 7.36 - $7.22(\mathrm{~m}, 6 \mathrm{H}), 6.90(\mathrm{~d}, J=9.0 \mathrm{~Hz}, 2 \mathrm{H}), 5.49(\mathrm{~s}, 2 \mathrm{H}), 5.23(\mathrm{~s}, 2 \mathrm{H}), 3.82(\mathrm{~s}, 3 \mathrm{H}), 1.36(\mathrm{~s}, 9 \mathrm{H}) \mathrm{ppm} .{ }^{13} \mathrm{C}$ NMR $\left(126 \mathrm{MHz}, \mathrm{CDCl}_{3}\right): \delta 180.8$ (C), 162.7 (C), 159.3 (C), 157.0 (C), 156.2 (C), 151.2 (C), 137.0 (C), 136.5 (C), $131.4(\mathrm{C}), 128.8(\mathrm{CH}), 128.6(\mathrm{CH}), 128.5(\mathrm{CH}), 128.3(\mathrm{CH}), 127.7(\mathrm{CH}), 127.6(\mathrm{CH}), 123.0(\mathrm{CH}), 113.9$ (CH), $88.3(\mathrm{C}), 55.5\left(\mathrm{CH}_{3}\right), 45.5\left(\mathrm{CH}_{2}\right), 44.5\left(\mathrm{CH}_{2}\right), 40.4(\mathrm{C}), 29.2\left(\mathrm{CH}_{3}\right) \mathrm{ppm}$. HRMS (ESI): $\mathrm{m} / \mathrm{z}$ calculated for $\mathrm{C}_{31} \mathrm{H}_{32} \mathrm{~N}_{5} \mathrm{O}_{3}[\mathrm{M}+\mathrm{H}]^{+}=522.2500$, found $=522.2500$. M.p.: $173^{\circ} \mathrm{C}$.

Methyl (6,8-dibenzyl-2-(tert-butyl)-5,7-dioxo-5,6,7,8-tetrahydropyrimido[4,5-d]pyrimidin-4yl)glycinate (5t) [HL149]<smiles>CC(=O)CNc1nc(C(C)(C)C)nc2c1c(=O)n(Cc1ccccc1)c(=O)n2Cc1ccccc1</smiles>

The general procedure $C$ was applied using $N^{\prime}$-(1,3-dibenzyl-2,6-dioxo1,2,3,6-tetrahydropyrimidin-4-yl)pivalimidamide $(\mathbf{1 g}, 195 \mathrm{mg}, 0.5 \mathrm{mmol}$, 1.0 equiv) and methyl 2 -isocyanoacetate $(93 \mu \mathrm{L}, 1.0 \mathrm{mmol}, 2.0$ equiv). The compound was purified by manual flash chromatography using cHex:EtOAc (8:1) as eluent. The title compound was isolated as a clear oil (34 mg, 14\%). Unknown compound according to a Sci-finder search.

$\mathbf{R}_{\mathbf{F}}=0.39$ (EtOAc : c-Hex : NEt $\left.t_{3}=1: 4: 0.01\right) .{ }^{1} \mathbf{H}$ NMR $\left(300 \mathrm{MHz}, \mathrm{CDCl}_{3}\right): \delta 9.13(\mathrm{t}, \mathrm{J}=5.6,1 \mathrm{H}), 7.53-7.41$ $(\mathrm{m}, 4 \mathrm{H}), 7.34-7.20(\mathrm{~m}, 6 \mathrm{H}), 5.45(\mathrm{~s}, 2 \mathrm{H}), 5.19(\mathrm{~s}, 2 \mathrm{H}), 4.28(\mathrm{~d}, J=5.6 \mathrm{~Hz}, 2 \mathrm{H}), 3.75(\mathrm{~s}, 3 \mathrm{H}), 1.30(\mathrm{~s}, 9 \mathrm{H})$ ppm. ${ }^{13} \mathrm{C} \mathrm{NMR}\left(126 \mathrm{MHz}, \mathrm{CDCl}_{3}\right.$ ): $\delta 180.4$ (C), 170.1 (C), 162.4 (C), 161.3 (C), 156.8 (C), 151.3 (C), 137.0 (C), $136.6(\mathrm{C}), 128.8(\mathrm{CH}), 128.7(\mathrm{CH}), 128.4(\mathrm{CH}), 128.3(\mathrm{CH}), 127.6(\mathrm{CH}), 127.6(\mathrm{CH}), 88.6(\mathrm{C}), 52.2$ $\left(\mathrm{CH}_{3}\right), 45.5\left(\mathrm{CH}_{2}\right), 44.4\left(\mathrm{CH}_{2}\right), 42.7\left(\mathrm{CH}_{2}\right), 40.1(\mathrm{C}), 29.0\left(\mathrm{CH}_{3}\right)$ ppm. HRMS (ESI): $\mathrm{m} / z$ calculated for $\mathrm{C}_{27} \mathrm{H}_{30} \mathrm{~N}_{5} \mathrm{O}_{4}[\mathrm{M}+\mathrm{H}]^{+}=488.2260$, found $=488.2292$.

5-(tert-butylamino)-1,3-dimethyl-7-phenylpyrimido[4,5-d]pyrimidine-2,4(1H,3H)-dione (6a) [JC755]<smiles>Cn1c(=O)c2c(NC(C)(C)C)nc(-c3ccccc3)nc2n(C)c1=O</smiles>
The general procedure $C$ was applied using $N$-(1,3-dimethyl-2,6-dioxo1,2,3,6-tetrahydropyrimidin-4-yl)benzimidamide (1h, $129 \mathrm{mg}, 0.5 \mathrm{mmol}, 1.0$ equiv) and tert-butyl isocyanide (2a, $119 \mu \mathrm{L}, 1.0 \mathrm{mmol}, 2.0$ equiv). The compound was purified by manual flash chromatography using cHex:EtOAc (10:1) as eluent. The title compound was isolated as a white solid (150 mg, 89\%). Unknown compound according to a Sci-finder search.

$\mathbf{R}_{\mathbf{F}}=0.64$ (cHex : EtOAc : NEt $;$; 5:1:0.01). ${ }^{1} \mathbf{H}$ NMR (300 MHz, $\left.\mathrm{CDCl}_{3}\right): \delta 9.21$ (bs, $1 \mathrm{H}), 8.48(\mathrm{~d}, J=6.8 \mathrm{~Hz}, 2 \mathrm{H}), 7.58-7.42(\mathrm{~m}, 3 \mathrm{H}), 3.73(\mathrm{~s}, 3 \mathrm{H}), 3.43(\mathrm{~s}, 3 \mathrm{H})$, 1.62 (s, 9H) ppm. ${ }^{13} \mathrm{C} \mathrm{NMR}\left(101 \mathrm{MHz}, \mathrm{CDCl}_{3}\right): \delta 165.1$ (C), 163.1 (C), 161.2 (C), 157.4 (C), 151.4 (C), 137.5 (C), $131.5(\mathrm{CH}), 128.9(\mathrm{CH}), 128.3(\mathrm{CH}), 88.7$ (C), $52.5(\mathrm{C}), 29.4\left(\mathrm{CH}_{3}\right), 29.1\left(\mathrm{CH}_{3}\right), 27.8\left(\mathrm{CH}_{3}\right)$ ppm. HRMS (ESI): $m / z$ calculated for $\mathrm{C}_{18} \mathrm{H}_{21} \mathrm{~N}_{5} \mathrm{O}_{2} \mathrm{Na}[\mathrm{M}+\mathrm{Na}]^{+}=362.1587$, found $=362.1568$. M.p.: $242{ }^{\circ} \mathrm{C}$.

\section{5-(Cyclohexylamino)-1,3-dimethyl-7-phenylpyrimido[4,5-d]pyrimidine-2,4(1H,3H)-dione [JC933]}

(6b)

The general procedure $\mathrm{C}$ was applied using $\mathrm{N}$-(1,3-dimethyl-2,6-dioxo-1,2,3,6-tetrahydropyrimidin-4yl)benzimidamide ( $1 \mathrm{~h}, 129 \mathrm{mg}, 0.5 \mathrm{mmol}, 1.0$ equiv) and cyclohexyl isocyanide ( $124 \mu \mathrm{L}, 1.0 \mathrm{mmol}, 2.0$ equiv). The compound was purified by manual flash chromatography using heptane:EtOAc (10:1) as 
<smiles>Cn1c(=O)c2c(NC3CCCCC3)nc(-c3ccccc3)nc2n(C)c1=O</smiles>

eluent. The title compound was isolated as a white solid (182 $\mathrm{mg}, 98 \%$ ). Unknown compound according to a Sci-finder search.

$\mathbf{R}_{\mathbf{F}}=0.58$ (cHex : EtOAc : NEt $;$; 5:1:0.01). ${ }^{1} \mathbf{H}$ NMR (400 MHz, $\left.\mathrm{CDCl}_{3}\right) \delta 9.02$ (bd, $J=7.8 \mathrm{~Hz}, 1 \mathrm{H}), 8.47(\mathrm{dd}, J=1.7,8.1 \mathrm{~Hz}, 2 \mathrm{H}), 7.62-7.36(\mathrm{~m}, 3 \mathrm{H}), 4.34$ (dtd, $J=3.7,8.1,12.9 \mathrm{~Hz}, 1 \mathrm{H}), 3.73(\mathrm{~s}, 3 \mathrm{H}), 3.43(\mathrm{~s}, 3 \mathrm{H}), 2.15-2.02(\mathrm{~m}, 2 \mathrm{H})$, 1.86-1.76 (m, 2H), $1.69-1.65(\mathrm{~m}, 1 \mathrm{H}), 1.57-1.29(\mathrm{~m}, 5 \mathrm{H}) \mathrm{ppm} .{ }^{13} \mathrm{C}$ NMR (101 $\left.\mathrm{MHz}, \mathrm{CDCl}_{3}\right) \delta 165.9$ (C), 163.0 (C), 160.8 (C), 157.6 (C), 151.5 (C), 137.4 (C), $131.5(\mathrm{CH}), 128.9(\mathrm{CH}), 128.3(\mathrm{CH}), 88.3(\mathrm{C}), 49.5(\mathrm{CH}), 32.7\left(\mathrm{CH}_{2}\right), 29.4\left(\mathrm{CH}_{2}\right)$, $27.8\left(\mathrm{CH}_{2}\right), 25.7\left(\mathrm{CH}_{2}\right), 24.6\left(\mathrm{CH}_{2}\right)$ ppm. HRMS (ESI): $\mathrm{m} / z$ calculated for $\mathrm{C}_{20} \mathrm{H}_{24} \mathrm{~N}_{5} \mathrm{O}_{2}[\mathrm{M}+\mathrm{H}]^{+}=366.1925$, found $=366.1909$. M.p.: $199^{\circ} \mathrm{C}$.

\section{5-(Butylamino)-1,3-dimethyl-7-phenylpyrimido[4,5-d]pyrimidine-2,4(1H,3H)-dione (6c) [JC779A]}<smiles>CCCCNc1nc(-c2ccccc2)nc2c1c(=O)n(C)c(=O)n2C</smiles>
The general procedure $C$ was applied using $N$-(1,3-dimethyl-2,6-dioxo1,2,3,6-tetrahydropyrimidin-4-yl)benzimidamide (1h, $129 \mathrm{mg}, 0.5 \mathrm{mmol}$, 1.0 equiv) and $n$-butyl isocyanide (105 $\mu \mathrm{L}, 1.0 \mathrm{mmol}, 2.0$ equiv). The compound was purified by manual flash chromatography using cHex:EtOAc (10:1) as eluent. The title compound was isolated as a white solid (125 mg, 74\%). Unknown compound according to a Sci-finder search.

$\mathbf{R}_{\mathrm{F}}=0.53$ (cHex : EtOAc: $\left.\mathrm{NEt}_{3} ; 5: 1: 0.01\right) .{ }^{1} \mathrm{H}$ NMR $\left(400 \mathrm{MHz}, \mathrm{CDCl}_{3}\right) \delta 8.99$ (bt, $\left.J=5.7,1 \mathrm{H}\right), 8.46$ (dd, $J=$ 1.5, 8.1 Hz, 2H), $7.55-7.41(\mathrm{~m}, 3 \mathrm{H}), 3.71(\mathrm{q}, J=6.6 \mathrm{~Hz}, 2 \mathrm{H}), 3.70(\mathrm{~s}, 3 \mathrm{H}), 3.40(\mathrm{~s}, 3 \mathrm{H}), 1.70(\mathrm{tt}, J=6.3$, $7.3 \mathrm{~Hz}, 2 \mathrm{H}), 1.49(\mathrm{dt}, 7.3,14.9 \mathrm{~Hz}, 2 \mathrm{H}), 0.99(\mathrm{t}, J=7.3 \mathrm{~Hz}, 3 \mathrm{H}) \mathrm{ppm} .{ }^{13} \mathrm{C} \mathrm{NMR}\left(101 \mathrm{MHz}, \mathrm{CDCl}_{3}\right) \delta 165.8$ (C), 162.9 (C), 161.6 (C), 157.4 (C), 151.4 (C), 137.2 (C), 131.5 (CH), 128.8 (CH), 128.2 (CH), 88.4 (C), 40.6 $\left(\mathrm{CH}_{2}\right), 31.5\left(\mathrm{CH}_{2}\right), 29.4(\mathrm{CH}), 27.8(\mathrm{CH}), 20.2\left(\mathrm{CH}_{2}\right), 13.8\left(\mathrm{CH}_{3}\right)$ ppm. HRMS (ESI): $\mathrm{m} / z$ calculated for $\mathrm{C}_{18} \mathrm{H}_{21} \mathrm{~N}_{5} \mathrm{O}_{2} \mathrm{Na}[\mathrm{M}+\mathrm{Na}]^{+}=362.1587$, found $=362.1570$. M.p.: $160^{\circ} \mathrm{C}$.

\section{1,3-dimethyl-5-[(2-morpholinoethyl)amino]-7-phenylpyrimido[4,5-d]pyrimidine-2,4(1H,3H)-dione} (6d) [JC784B]<smiles>Cn1c(=O)c2c(NCCN3CCOCC3)nc(-c3ccccc3)nc2n(C)c1=O</smiles>

The general procedure $\mathrm{C}$ was applied using $\mathrm{N}$-(1,3-dimethyl-2,6-dioxo1,2,3,6-tetrahydropyrimidin-4-yl)benzimidamide (1 $\mathbf{h}, 129 \mathrm{mg}, 0.5 \mathrm{mmol}$, 1.0 equiv) and 2-morpholinoethyl isocyanide $(140 \mathrm{mg}, 1.0 \mathrm{mmol}, 2.0$ equiv). The compound was purified by manual flash chromatography using cHex:EtOAc: NEt $_{3}$ (gradient $=$ 20:1:0.01-4:1:0.01) as eluent. The title compound $\mathbf{6 d}$ was isolated as a white solid (101 $\mathrm{mg}, 51 \%)$. The starting material was recovered via this isolation $(63 \mathrm{mg}, 49 \%)$. Unknown compound according to a Sci-finder search.

$\mathbf{R}_{\mathbf{F}}=0.09$ (cHex : EtOAc: NEt $;$; 15:1:0.01). ${ }^{1} \mathbf{H}$ NMR (300 MHz, CDCl $) \delta 9.23$ (bt, $J=5.5 \mathrm{~Hz}, 1 \mathrm{H}$ ), 8.47 $(\mathrm{d}, J=6.2 \mathrm{~Hz}, 2 \mathrm{H}), 7.59-7.37(\mathrm{~m}, 3 \mathrm{H}), 3.85(\mathrm{q}, J=6.0 \mathrm{~Hz}, 2 \mathrm{H}), 3.76(\mathrm{t}, J=4.6 \mathrm{~Hz}, 4 \mathrm{H}), 3.73(\mathrm{~s}, 3 \mathrm{H}), 3.44$ $(\mathrm{s}, 3 \mathrm{H}), 2.69(\mathrm{t}, 6.3 \mathrm{~Hz}, 2 \mathrm{H}), 2.56(\mathrm{t}, J=4.6 \mathrm{~Hz}, 4 \mathrm{H}) \mathrm{ppm} .{ }^{13} \mathrm{C} \mathrm{NMR}\left(101 \mathrm{MHz}, \mathrm{CDCl}_{3}\right) \delta 165.8(\mathrm{C}), 162.7$ (C), $161.5(\mathrm{C}), 157.4(\mathrm{C}), 151.5(\mathrm{C}), 137.2(\mathrm{C}), 131.6(\mathrm{CH}), 128.8(\mathrm{CH}), 128.3(\mathrm{CH}), 88.6(\mathrm{C}), 67.0\left(\mathrm{CH}_{2}\right)$, $57.2\left(\mathrm{CH}_{2}\right), 53.6\left(\mathrm{CH}_{2}\right), 37.7\left(\mathrm{CH}_{2}\right), 29.4\left(\mathrm{CH}_{3}\right), 27.8\left(\mathrm{CH}_{3}\right)$ ppm. HRMS (ESI): $m / z$ calculated for $\mathrm{C}_{20} \mathrm{H}_{25} \mathrm{~N}_{6} \mathrm{O}_{3}[\mathrm{M}+\mathrm{H}]^{+}=397.1983$, found $=397.1989$. M.p.: $156^{\circ} \mathrm{C}$.

\section{5-[(4-methoxyphenyl)amino]-1,3-dimethyl-7-phenylpyrimido[4,5-d]pyrimidine-2,4(1H,3H)-dione (6e) [JC959]}

The general procedure $\mathrm{C}$ was applied using $\mathrm{N}$-(1,3-dimethyl-2,6-dioxo-1,2,3,6-tetrahydropyrimidin-4yl)benzimidamide (1h, $129 \mathrm{mg}, 0.5 \mathrm{mmol}, 1.0$ equiv) and 4-methoxyphenyl isocyanide (133 $\mathrm{mg}, 1.0$ mmol, 2.0 equiv). The compound was purified by manual flash chromatography using cHex:EtOAc: $\mathrm{NEt}_{3}$ 
<smiles>COc1ccc(Nc2nc(-c3ccccc3)nc3c2c(=O)n(C)c(=O)n3C)cc1</smiles>

(4:1:0.01) as eluent. The title compound was isolated as a bright yellow solid (40 mg, 21\%). Unknown compound according to a Sci-finder search.

$\mathbf{R}_{\mathrm{F}}=0.27$ (cHex : EtOAc $\left.=4: 1\right) .{ }^{1} \mathbf{H}$ NMR $\left(500 \mathrm{MHz}, \mathrm{CDCl}_{3}\right) \delta 11.01(\mathrm{br} \mathrm{s}$, $1 \mathrm{H}), 8.46-8.39(\mathrm{~m}, 2 \mathrm{H}), 7.73-7.66(\mathrm{~m}, 2 \mathrm{H}), 7.56-7.49(\mathrm{~m}, 1 \mathrm{H}), 7.48$ $(\mathrm{dd}, J=8.2,6.5 \mathrm{~Hz}, 2 \mathrm{H}), 7.00-6.94(\mathrm{~m}, 2 \mathrm{H}), 3.86(\mathrm{~s}, 3 \mathrm{H}), 3.75(\mathrm{~s}, 3 \mathrm{H})$, $3.46(\mathrm{~s}, 3 \mathrm{H}) \mathrm{ppm} .{ }^{13} \mathrm{C}$ NMR $\left(126 \mathrm{MHz}, \mathrm{CDCl}_{3}\right) \delta 166.0$ (C), 162.9 (C), 159.4 (C), 157.4 (C), 156.5 (C), 151.2 (C), 136.9 (C), 131.8 (CH), 131.1 (C), 129.0 $(\mathrm{CH}), 128.4(\mathrm{CH}), 123.4(\mathrm{CH}), 114.0(\mathrm{CH}), 88.6(\mathrm{C}), 55.5\left(\mathrm{CH}_{3}\right), 29.5\left(\mathrm{CH}_{3}\right), 28.0\left(\mathrm{CH}_{3}\right)$ ppm. HRMS (ESI): $\mathrm{m} / z$ calculated for $\mathrm{C}_{21} \mathrm{H}_{20} \mathrm{~N}_{5} \mathrm{O}_{3}[\mathrm{M}+\mathrm{H}]^{+}=390.1561$, found $=390.1570$. M.p.: $209-213^{\circ} \mathrm{C}$.

\section{5-amino-1,3-dibenzyl-7-phenylpyrimido[4,5-d]pyrimidine-2,4(1H,3H)-dione (7)}<smiles>CC(C)(C)Nc1nc(-c2ccccc2)nc2[nH]c(=O)[nH]c(=O)c12</smiles>

To a solution of 1,3-dibenzyl-5-(tert-butylamino)-7-methylpyrimido[4,5d]pyrimidine-2,4(1H,3H)-dione (5a, $0.246 \mathrm{~g}, 0.5 \mathrm{mmol}, 1.0$ equiv) in dry methanol $(15 \mathrm{~mL})$ was added ammonium formate $(0.630 \mathrm{~g}, 10 \mathrm{mmol}, 20$ equiv) and $\mathrm{Pd} / \mathrm{C}(10 \%, 0.08 \mathrm{~g}, 7.5 \mathrm{~mol} \%)$ under argon. The resulting mixture was refluxed for $48 \mathrm{~h}$. After TLC indicated full conversion, the mixture was filtered over a pad of diatomaceous earth. The solid residue was washed 4 times with $\mathrm{MeOH}(4 \times 50 \mathrm{~mL})$ The filtrate was diluted with $\mathrm{DCM}(80 \mathrm{~mL})$, washed with sat. $\mathrm{NaHCO}_{3}(100 \mathrm{~mL})$, dried over $\mathrm{Na}_{2} \mathrm{SO}_{4}$, and concentrated in vacuo. The title compound was isolated without further purification as a white solid $(0.154 \mathrm{~g}, 50 \%)$. Unknown compound according to a Sci-finder search.

$\mathbf{R}_{\mathbf{F}}=0.29$ (cHex : EtOAc: NEt $\left.{ }_{3}\right)=1: 1: 0.01 .{ }^{1} \mathbf{H}$ NMR $\left(500 \mathrm{MHz}, \mathrm{DMSO}-\mathrm{d}_{6}\right) \delta 11.69$ (brs, 1H), 11.48 (brs, $1 \mathrm{H}), 8.98(\mathrm{~s}, 1 \mathrm{H}), 8.36(\mathrm{dd}, J=7.8,1.9 \mathrm{~Hz}, 2 \mathrm{H}), 7.64-7.51(\mathrm{~m}, 3 \mathrm{H}), 1.57(\mathrm{~s}, 9 \mathrm{H}) \mathrm{ppm} .{ }^{13} \mathrm{C} \mathrm{NMR}(125 \mathrm{MHz}$, DMSO-d $_{6}$ ) $\delta 164.7$ (C), 164.6 (C), 160.5 (C), 159.1 (C), 150.1 (C), 137.0 (C), 131.6 (CH), 128.6 (CH), 128.2 $(\mathrm{CH}), 87.9$ (C), 51.9 (C), $28.7\left(\mathrm{CH}_{3}\right) \mathrm{ppm}$. HRMS (ESI): $\mathrm{m} / z$ calculated for $\mathrm{C}_{16} \mathrm{H}_{18} \mathrm{~N}_{5} \mathrm{O}_{2}[\mathrm{M}+\mathrm{H}]^{+}=312.1455$, found $=312.1471$. M.p.: $257-259^{\circ} \mathrm{C}$.

\section{5-amino-1,3-dibenzyl-7-phenylpyrimido[4,5-d] pyrimidine-2,4(1H,3H)-dione (8) [JC890]}<smiles>Nc1nc(-c2ccccc2)nc2c1c(=O)n(Cc1ccccc1)c(=O)n2Cc1ccccc1</smiles>

To a solution of 1,3-dibenzyl-5-(tert-butylamino)-7-methylpyrimido[4,5d]pyrimidine-2,4(1H,3H)-dione ( $3 a, 0.246 \mathrm{~g}, 0.5 \mathrm{mmol}, 1.0$ equiv) in DCM $(5 \mathrm{~mL})$ was added triflic acid $(0.139 \mathrm{~g}, 2.5 \mathrm{mmol}, 5.0$ equiv), after which the mixture was stirred at room temperature for $2 \mathrm{~h}$. The resulting mixture was diluted with $\mathrm{DCM}(20 \mathrm{~mL})$, and washed twice with saturated $\mathrm{NaHCO}_{3}(50$ $\mathrm{mL}$ ). The solution was dried over $\mathrm{Na}_{2} \mathrm{SO}_{4}$, and concentrated in vacuo. The resulting solid was dried overnight on a lyophilizer to afford a fluffy white solid $(0.218 \mathrm{~g}, 100 \%)$. Unknown compound according to a Sci-finder search.

${ }^{1} \mathrm{H}$ NMR $\left(300 \mathrm{MHz}, \mathrm{DMSO}-\mathrm{d}_{6}\right): \delta 8.42(\mathrm{bd}, J=3.6 \mathrm{~Hz}, 1 \mathrm{H}), 8.37-8.30(\mathrm{~m}, 3 \mathrm{H}), 8.32(\mathrm{bd}, J=1.8 \mathrm{~Hz}, 1 \mathrm{H})$, $7.59-7.45(\mathrm{~m}, 3 \mathrm{H}), 7.40(\mathrm{~d}, J=6.9 \mathrm{~Hz}, 2 \mathrm{H}), 7.36-7.20(\mathrm{~m}, 7 \mathrm{H}), 5.48(\mathrm{~s}, 2 \mathrm{H}), 5.11(\mathrm{~s}, 2 \mathrm{H}) \mathrm{ppm} .{ }^{13} \mathrm{C}$ NMR (101 MHz, DMSO-d d $\left._{6}\right)$ ס 165.1 (C), 163.1 (C), 161.9 (C), 157.4 (C), 150.9 (C), 137.4 (C), 137.0 (C), $136.6(\mathrm{C}), 131.9(\mathrm{CH}), 128.6(\mathrm{CH}), 128.5(\mathrm{CH}), 127.5(\mathrm{CH}), 127.4(\mathrm{CH}), 127.3(\mathrm{CH}), 127.3(\mathrm{CH}), 88.2(\mathrm{C})$, $45.3\left(\mathrm{CH}_{2}\right), 44.1\left(\mathrm{CH}_{2}\right) \mathrm{ppm}$ ( $2 \mathrm{CH}_{\mathrm{Ar}}$ missing due to overlapping signal). HRMS (ESI): $m / z$ calculated for $\mathrm{C}_{26} \mathrm{H}_{22} \mathrm{~N}_{5} \mathrm{O}_{2}[\mathrm{M}+\mathrm{H}]^{+}=436.1768$, found $=436.1768$. M.p.: $231^{\circ} \mathrm{C}$. 


\section{$5{ }^{1} \mathrm{H}$ and ${ }^{13} \mathrm{C}$ NMR Spectra}

1a, ${ }^{1} \mathrm{H}$ NMR (300 MHz, $\left.\mathrm{CDCl}_{3}\right)$ (JC721)

JC721_pp/1

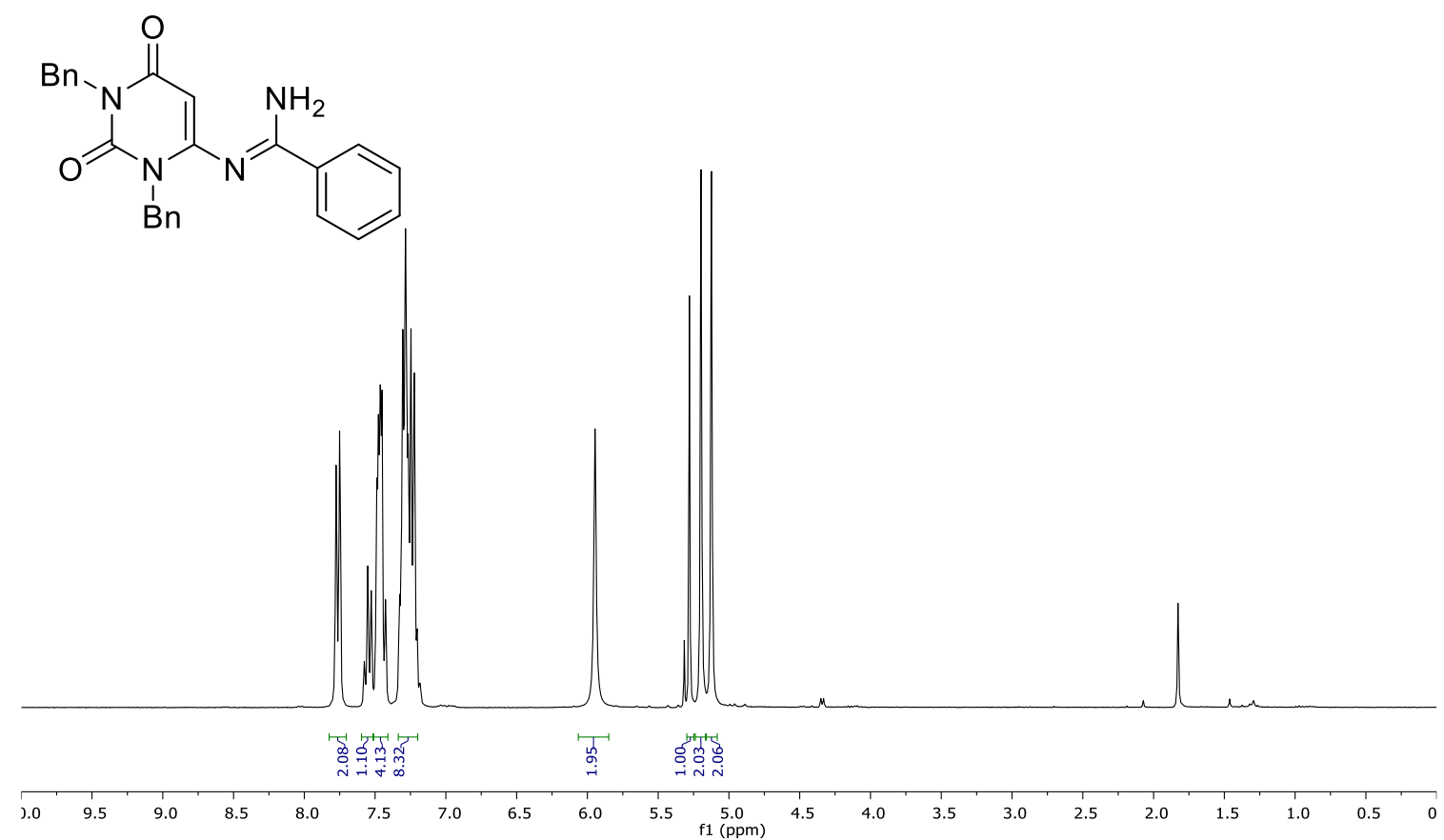

1a, $\left.{ }^{1} \mathrm{H} \mathrm{NMR} \mathrm{(125} \mathrm{MHz,} \mathrm{CDCl}_{3}\right)(\mathrm{JC721})$

BM-309-02-07-2015.12.fid

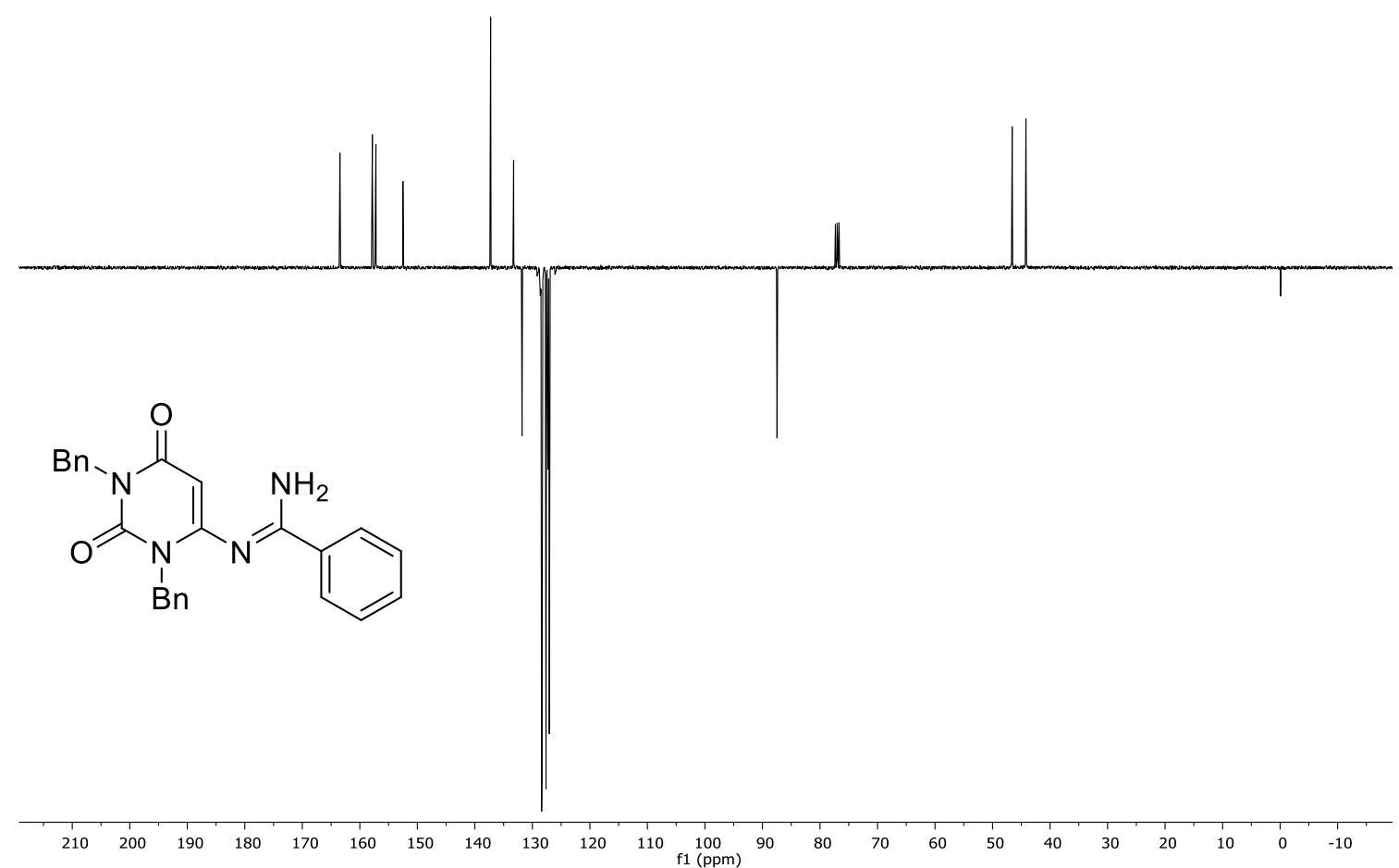


1b, ${ }^{1} \mathrm{H}$ NMR (300 $\left.\mathrm{MHz}, \mathrm{CDCl}_{3}\right)$

HL110.3.fid

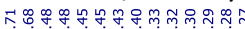

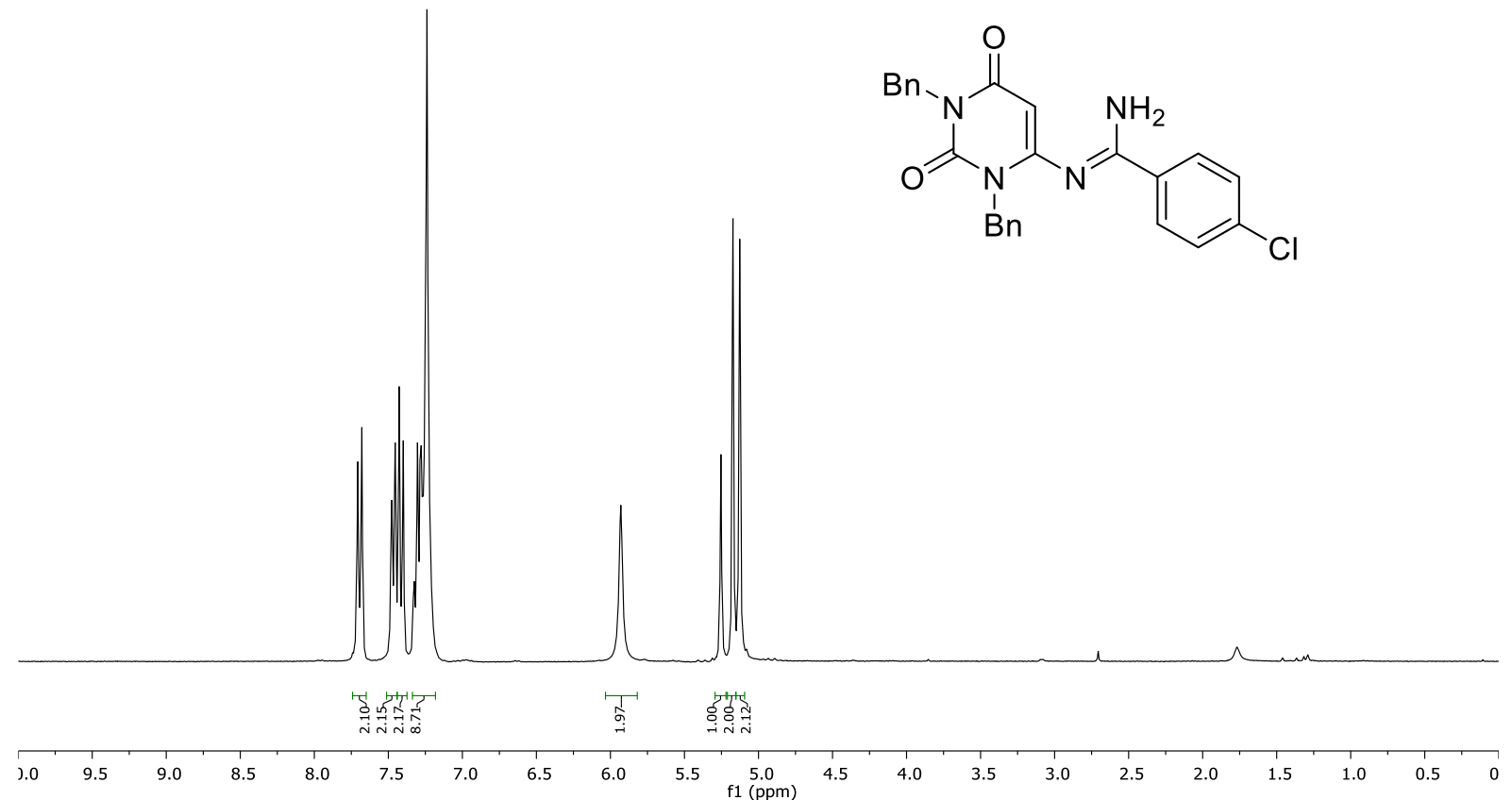

1b, ${ }^{1} \mathrm{H}$ NMR (125 MHz, $\mathrm{CDCl}_{3}$ )

HL110/1

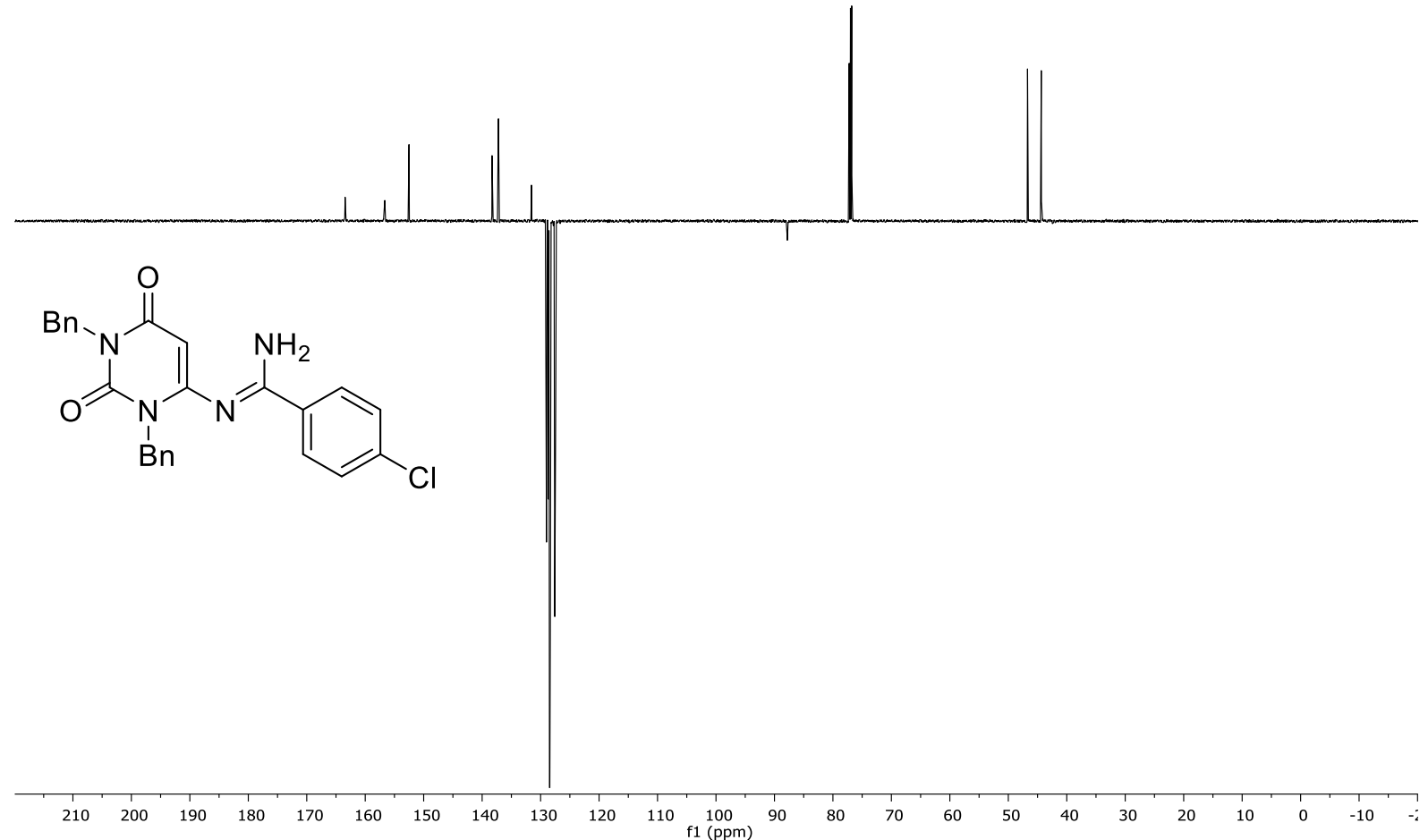


1c, ${ }^{1} \mathrm{H}$ NMR (300 MHz, $\left.\mathrm{CDCl}_{3}\right)$

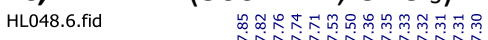

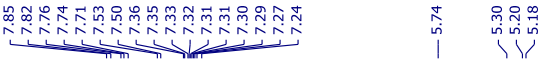

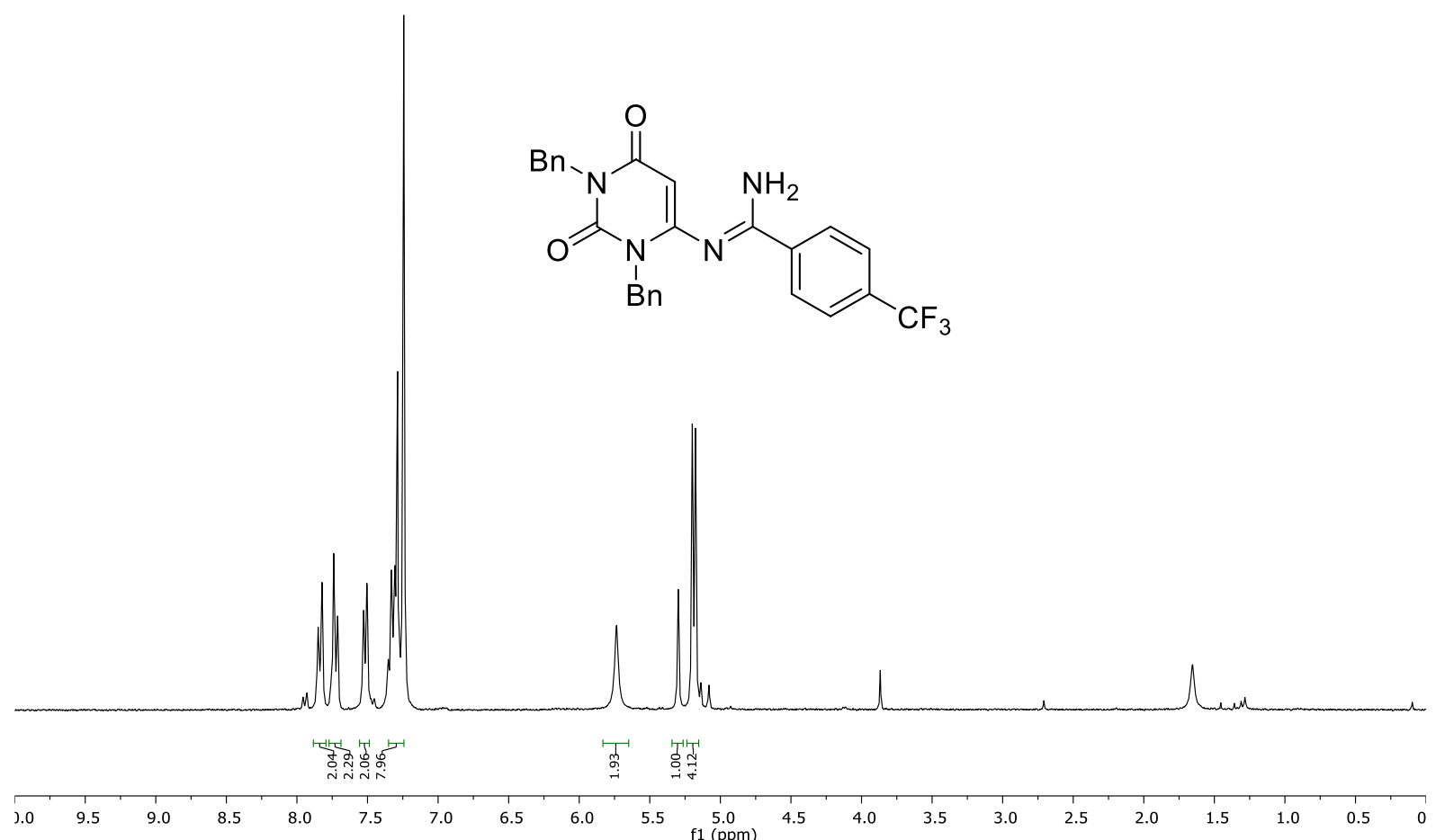

1c, ${ }^{1} \mathrm{H}$ NMR (125 MHz, $\left.\mathrm{CDCl}_{3}\right)$

HL048/1<smiles>N/C(=N\c1cc(=O)n(Cc2ccccc2)c(=O)n1Cc1ccccc1)c1ccc(C(F)(F)F)cc1</smiles>

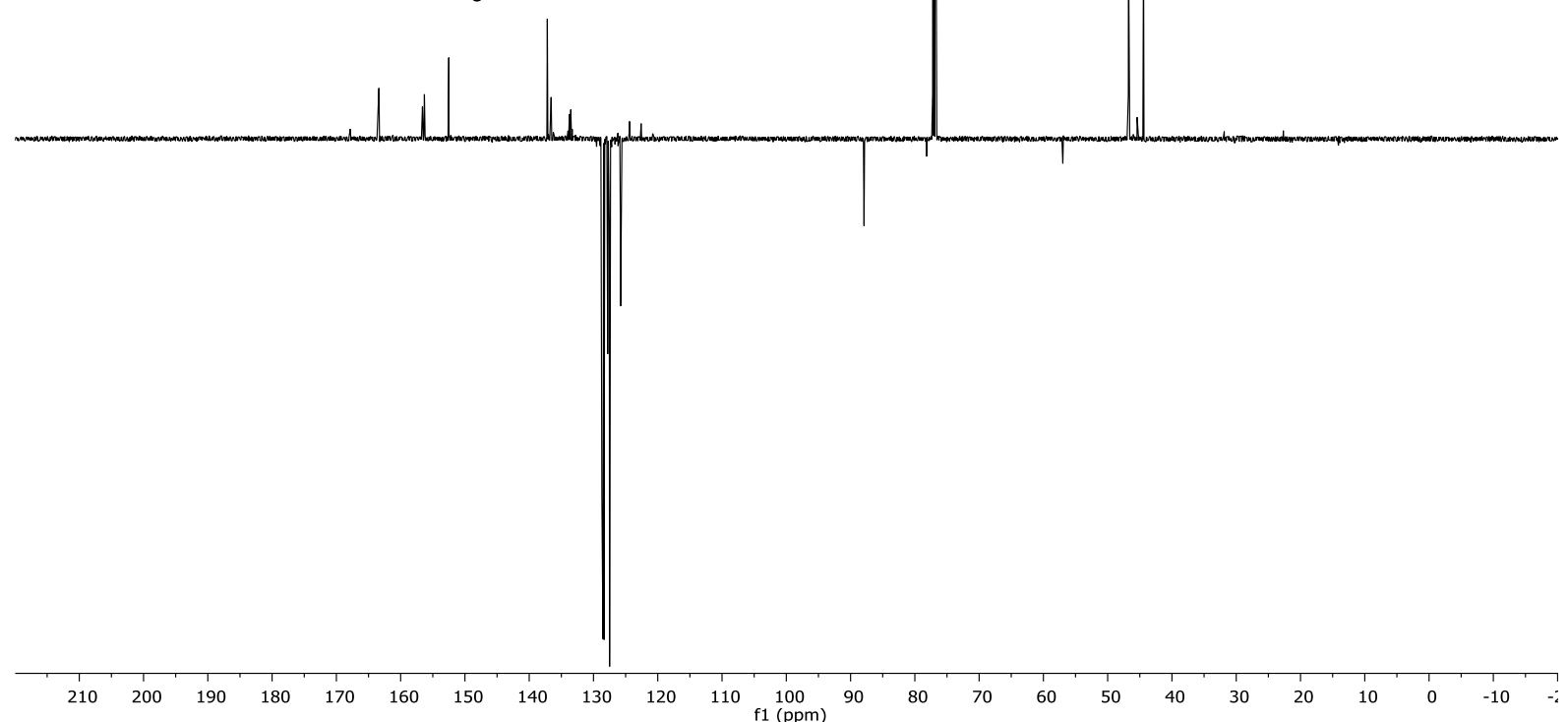


1d, ${ }^{1} \mathrm{H}$ NMR (300 MHz, $\left.\mathrm{CDCl}_{3}\right)$

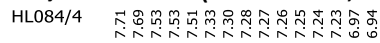

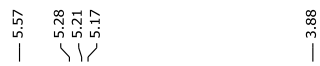

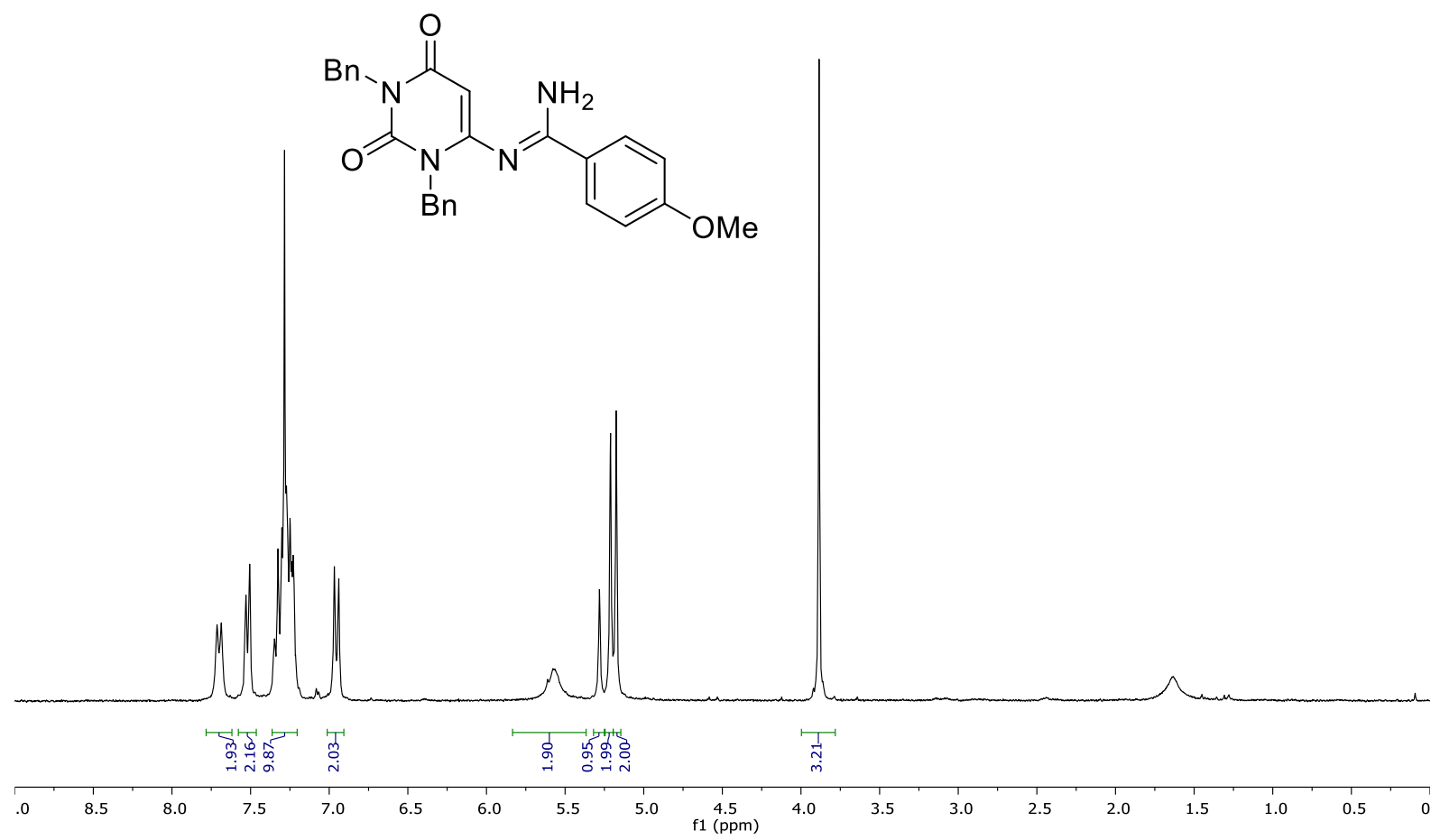

1d, ${ }^{1} \mathrm{H}$ NMR (151 MHz, $\mathrm{CDCl}_{3}$ )

HLO84/2<smiles>COc1ccc(/C(N)=N/c2cc(=O)n(Cc3ccccc3)c(=O)n2Cc2ccccc2)cc1</smiles>

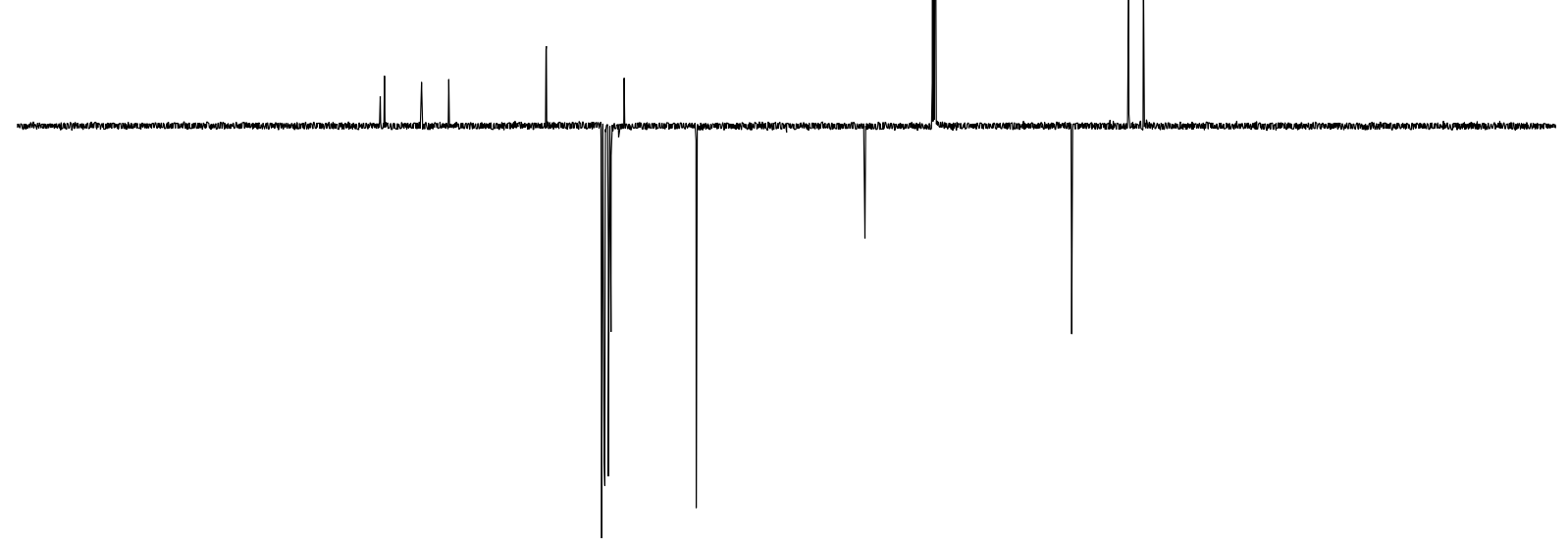

$\begin{array}{llllllllllllllllllllllllllll}210 & 200 & 190 & 180 & 170 & 160 & 150 & 140 & 130 & 120 & 110 & 100 & 90 & 80 & 70 & 60 & 50 & 40 & 30 & 20 & 10 & 0 & -10 & -\end{array}$ 
1e, ${ }^{1} \mathrm{H} \mathrm{NMR}\left(600 \mathrm{MHz}, \mathrm{CDCl}_{3}\right)$

HLo80/5
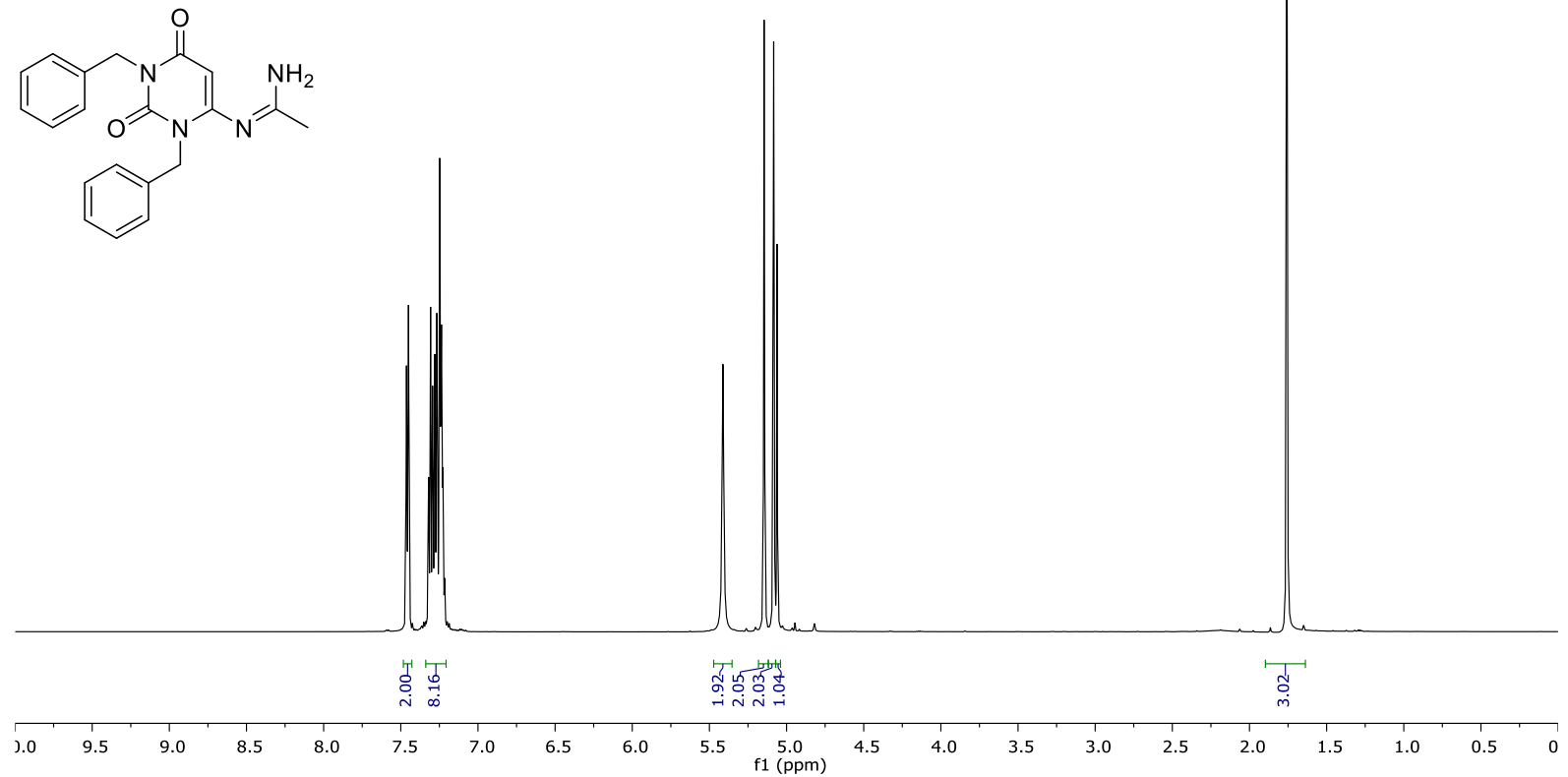

1e, ${ }^{1} \mathrm{H}$ NMR (151 MHz, $\mathrm{CDCl}_{3}$ ) HL080.8.fid

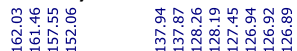
|广j
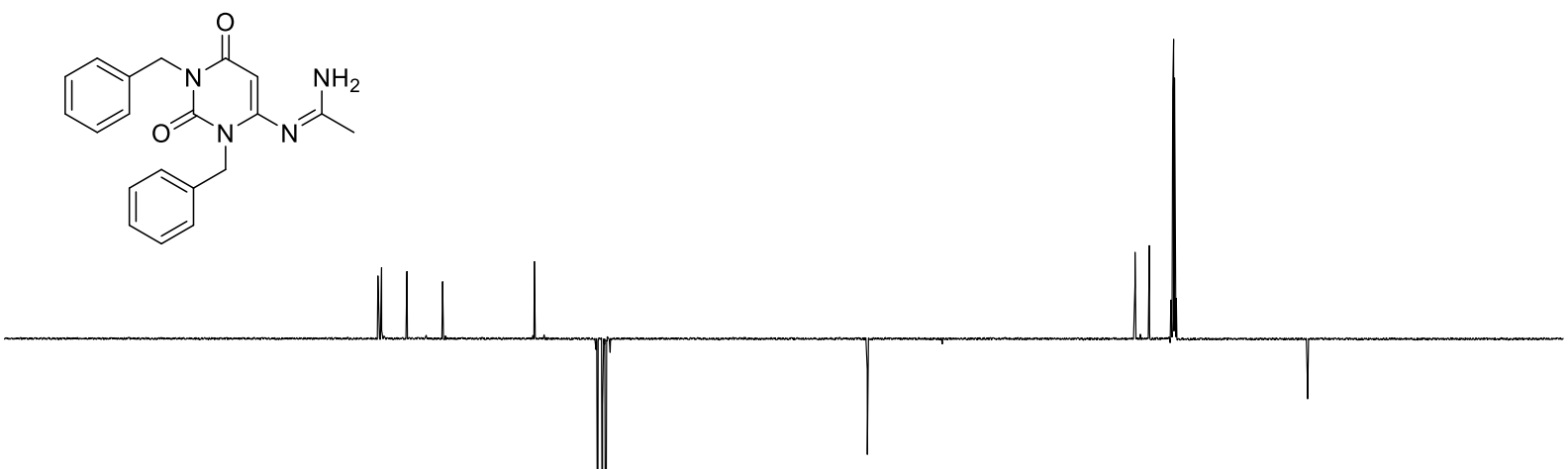

$\begin{array}{lllllllllllllllllllllllll}210 & 200 & 190 & 180 & 170 & 160 & 150 & 140 & 130 & 120 & 110 & \begin{array}{c}100 \\ \mathrm{f} 1(\mathrm{ppm})\end{array} & 90 & 80 & 70 & 60 & 50 & 40 & 30 & 20 & 10 & 0 & -10 & -2\end{array}$ 


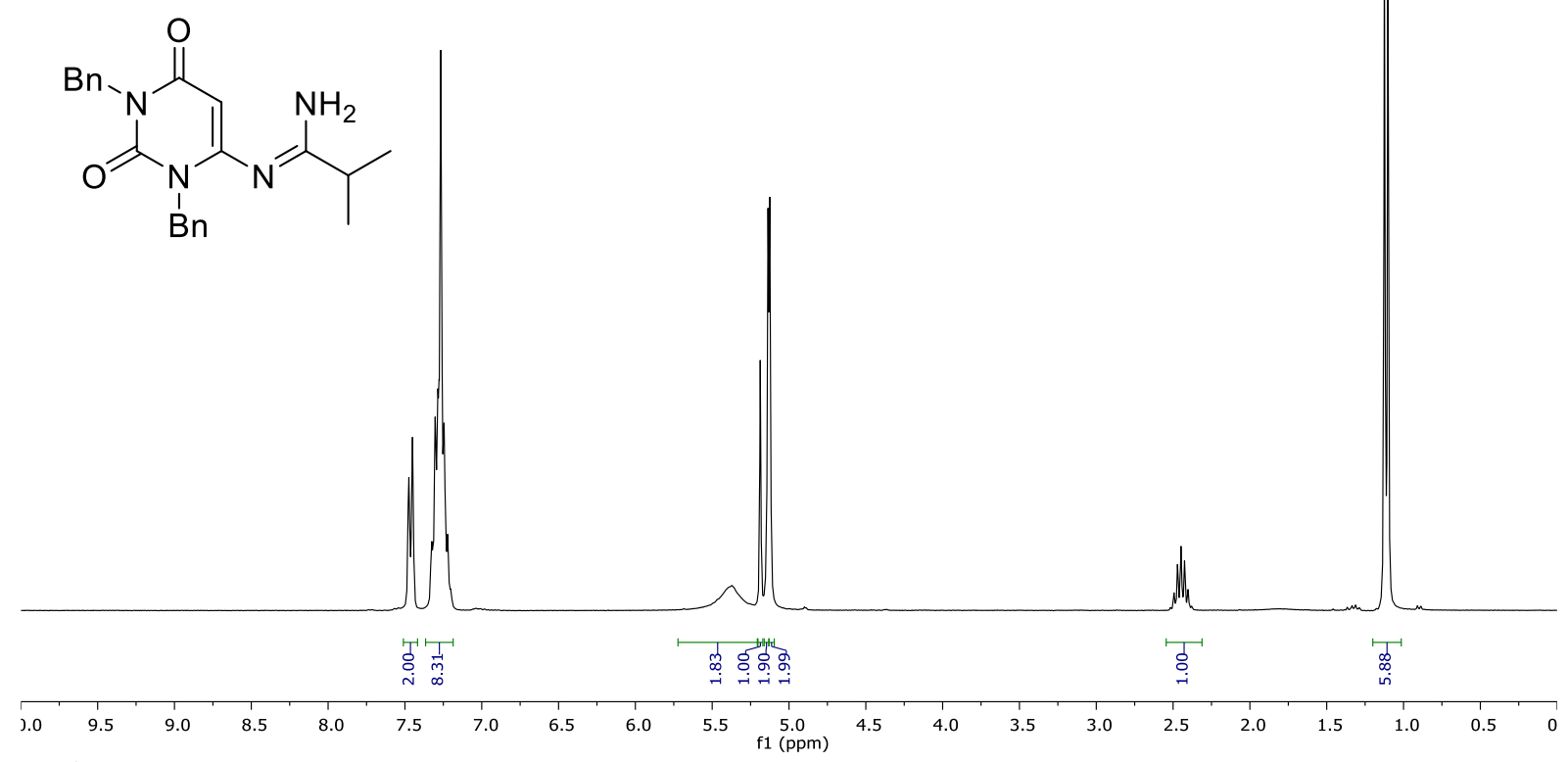

1f, ${ }^{1} \mathrm{H}$ NMR (125 MHz, $\mathrm{CDCl}_{3}$ )

HL095/2

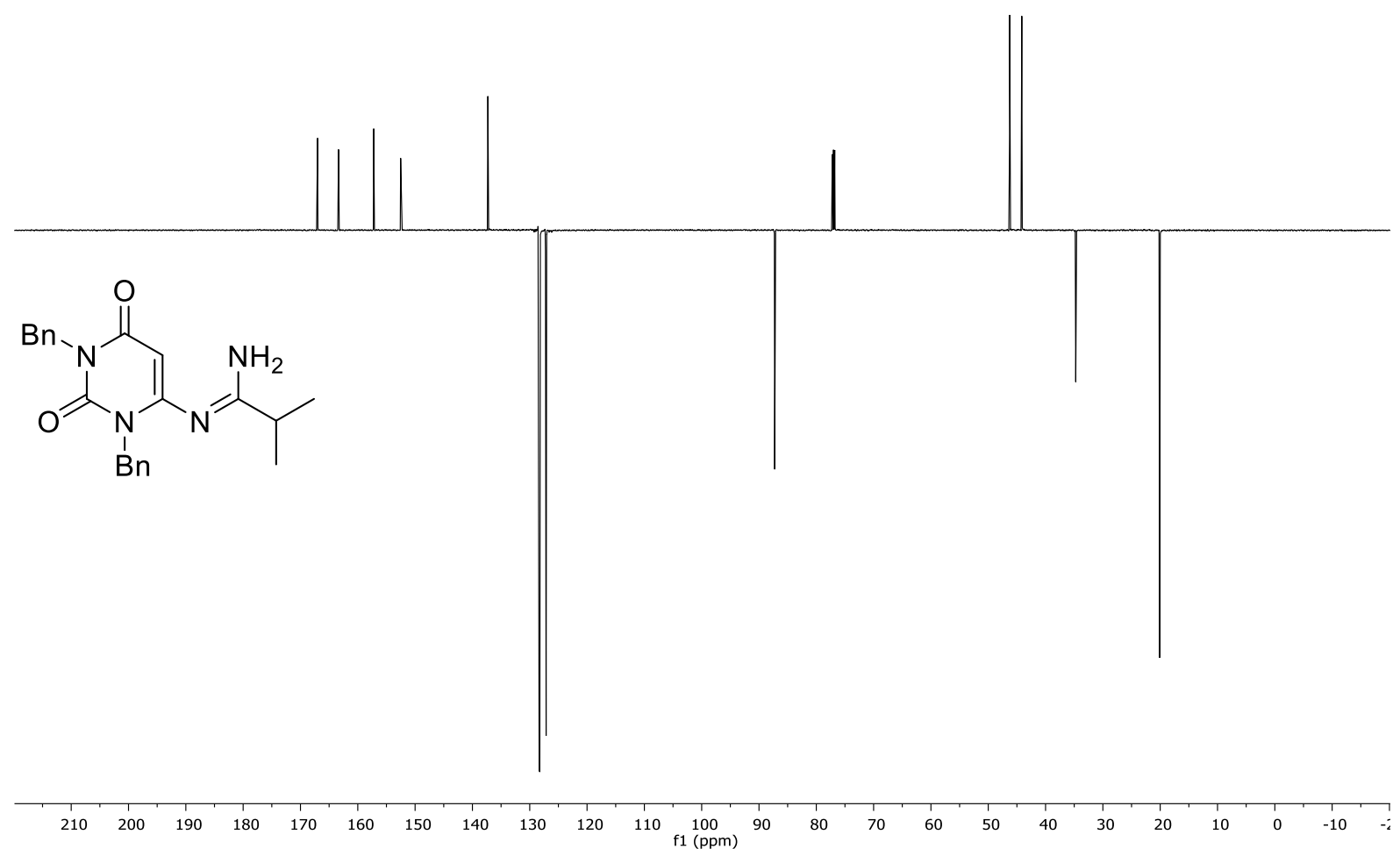


1g, ${ }^{1} \mathrm{H}$ NMR $\left(300 \mathrm{MHz}, \mathrm{CDCl}_{3}\right)$

HL069/4<smiles>CC(C)(C)/C(N)=N/c1cc(=O)n(Cc2ccccc2)c(=O)n1Cc1ccccc1</smiles>

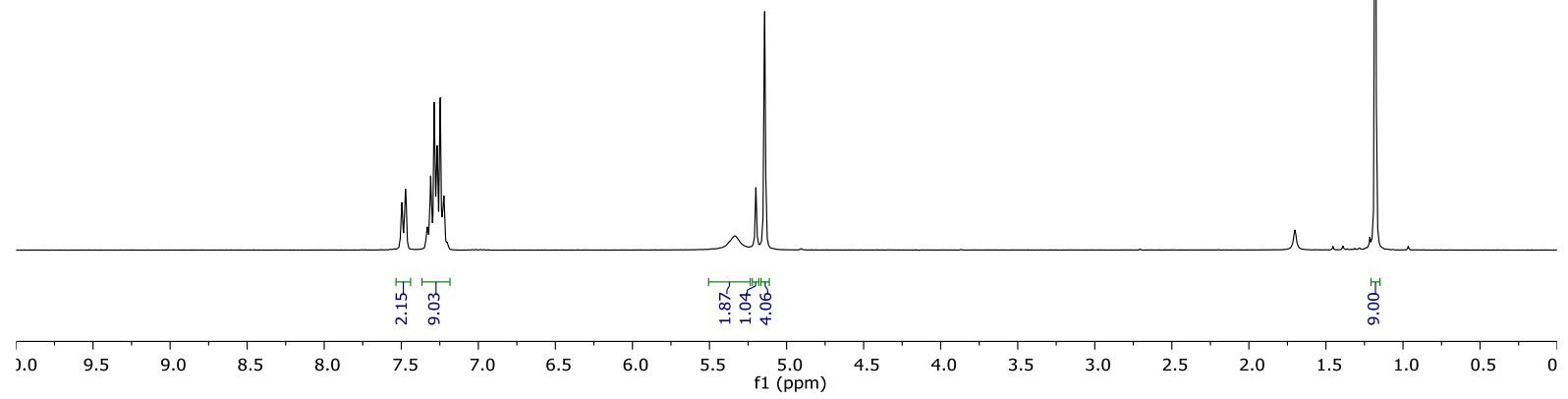

$1 \mathrm{~g},{ }^{1} \mathrm{H}$ NMR $\left(125 \mathrm{MHz}, \mathrm{CDCl}_{3}\right)$

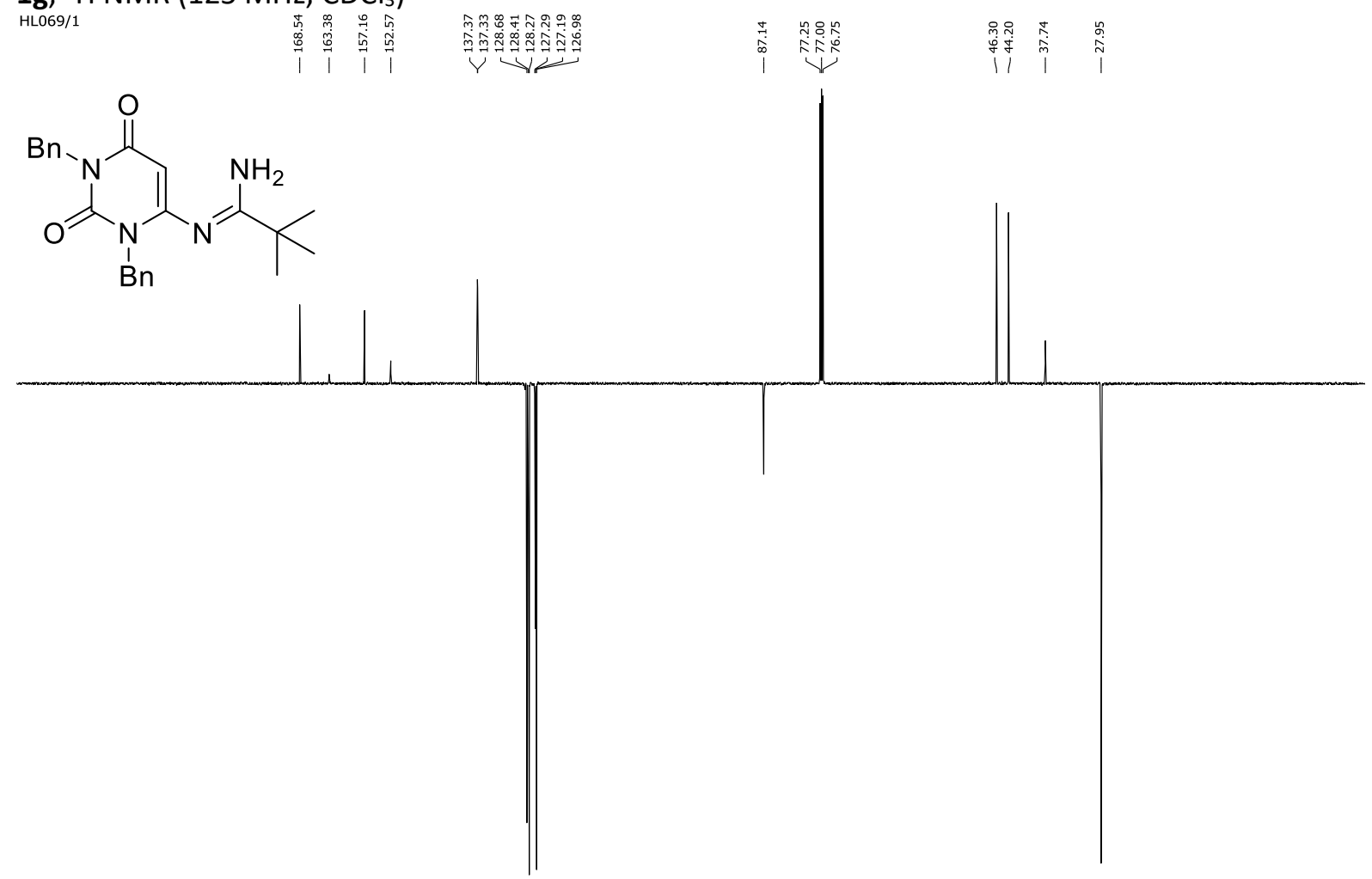

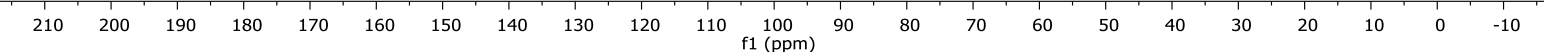


3a, ${ }^{1} \mathrm{H} \mathrm{NMR}\left(300 \mathrm{MHz}, \mathrm{CDCl}_{3}\right)$

HL062.3.fid<smiles>CC(C)(C)Nc1nc(-c2ccccc2)nc2c1c(=O)n(Cc1ccccc1)c(=O)n2Cc1ccccc1</smiles>

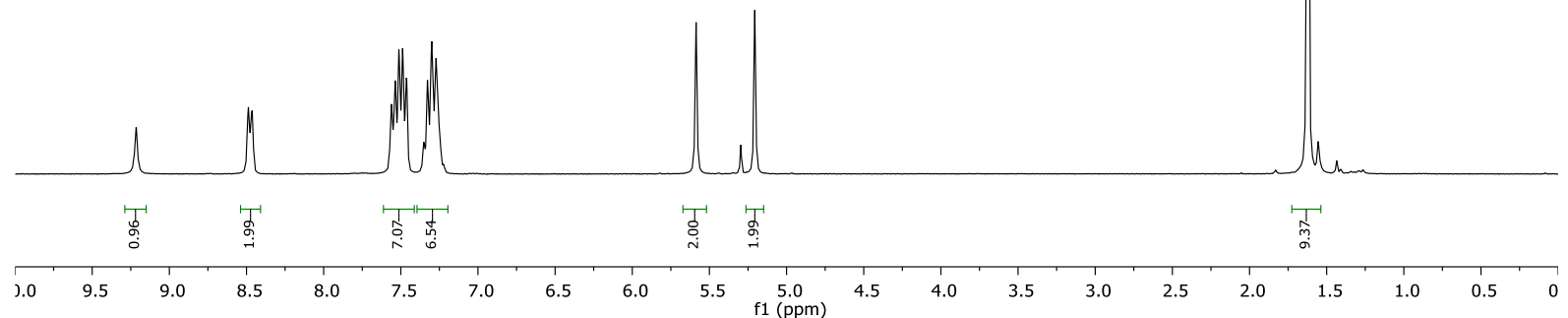

3a, ${ }^{1} \mathrm{H}$ NMR (126 MHz, $\mathrm{CDCl}_{3}$ )

JC720.11.fid

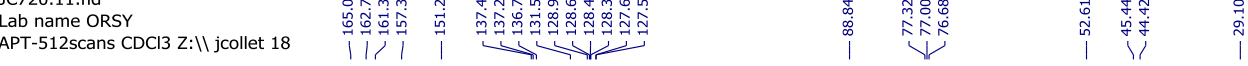

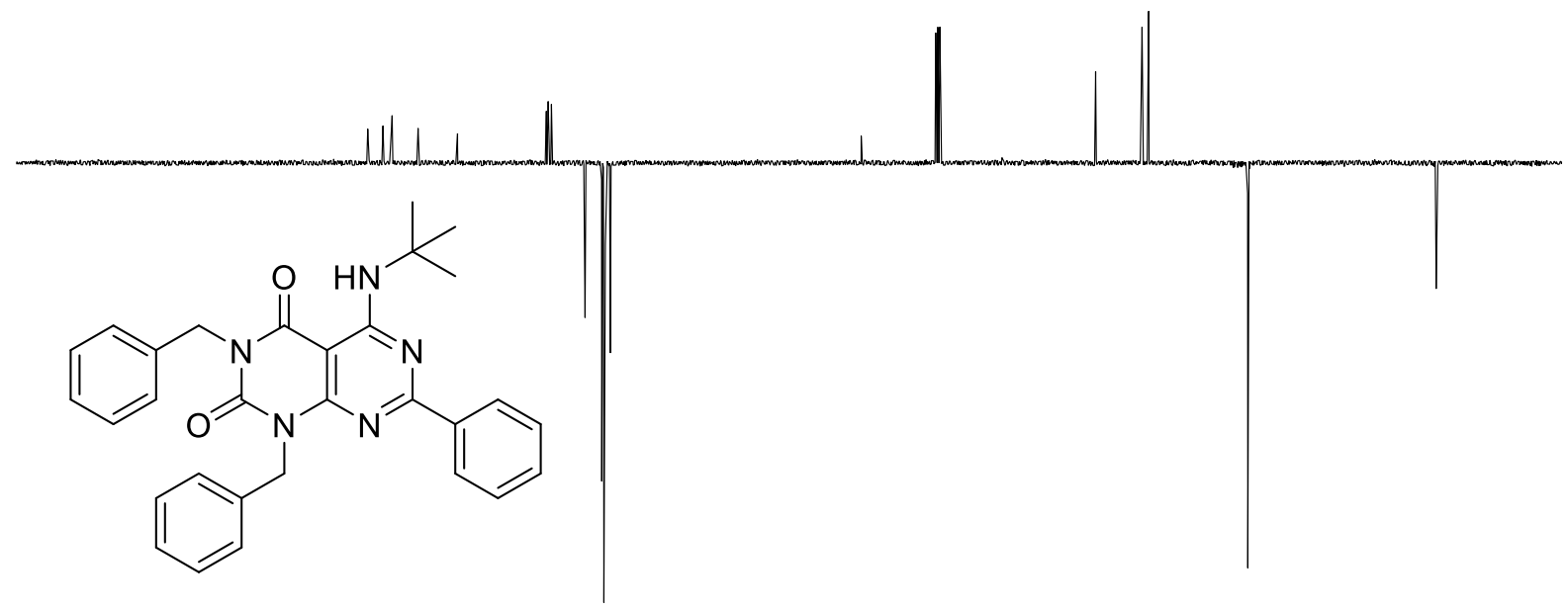

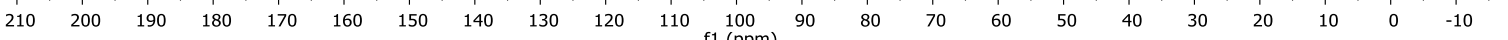


3b, ${ }^{1} \mathrm{H}$ NMR (500 MHz, $\mathrm{CDCl}_{3}$ )

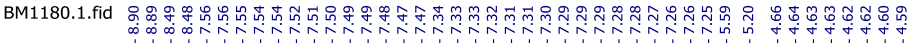<smiles>CC(C)Nc1nc(-c2ccccc2)nc2c1c(=O)n(Cc1ccccc1)c(=O)n2Cc1ccccc1</smiles>

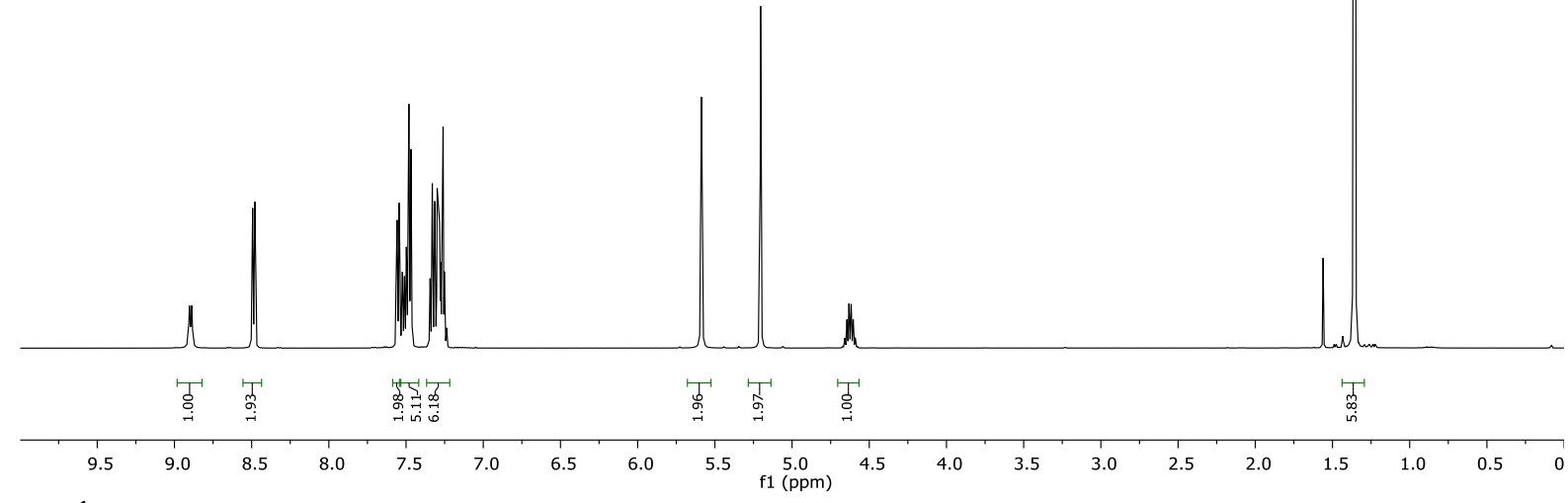

3b, ${ }^{1} \mathrm{H}$ NMR (126 MHz, $\mathrm{CDCl}_{3}$ )

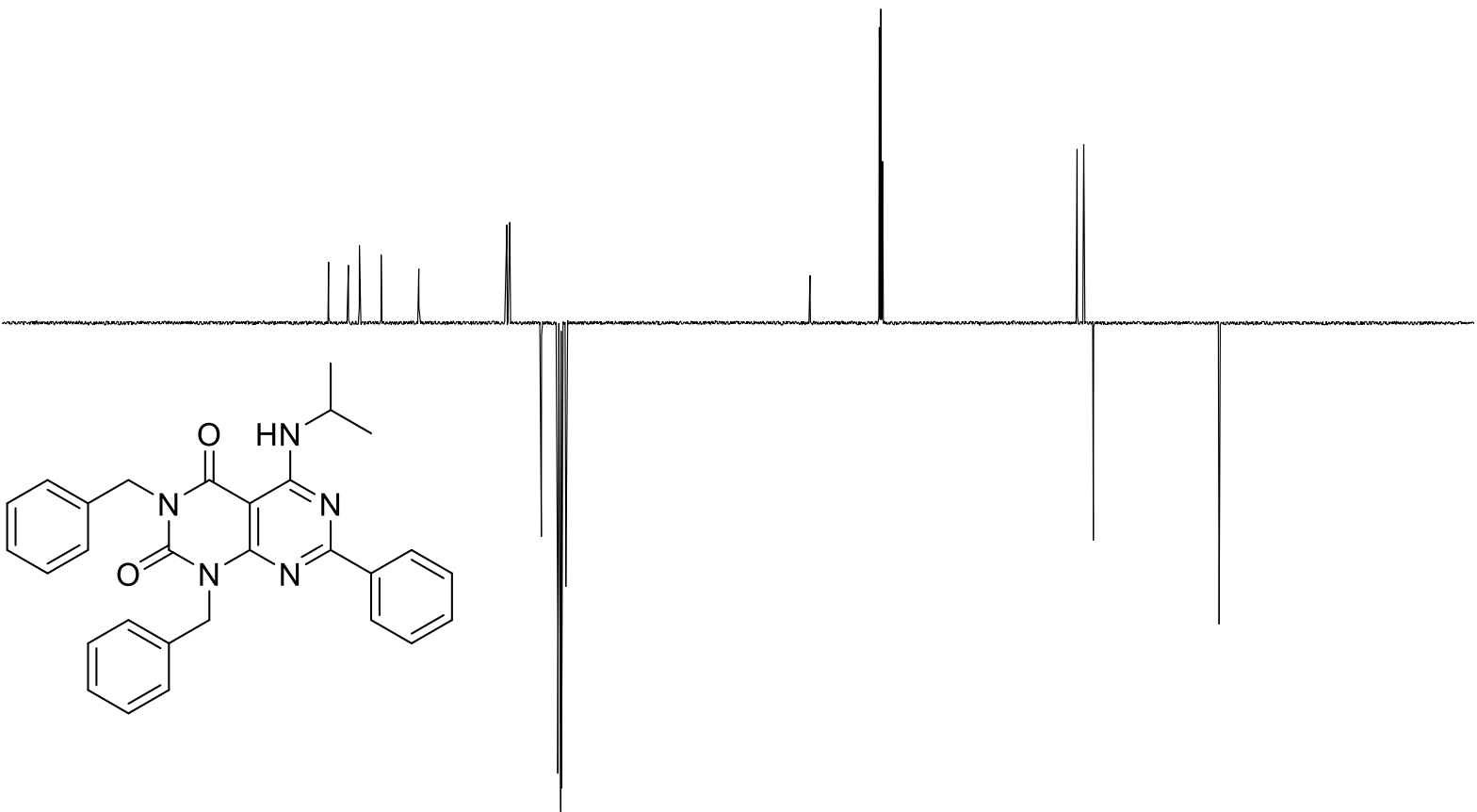

$\begin{array}{llllllllllllllllllllllllllllllllll}210 & 200 & 190 & 180 & 170 & 160 & 150 & 140 & 130 & 120 & 110 & 100 & 90 & 80 & 70 & 60 & 50 & 40 & 30 & 20 & 10 & 0 & -10\end{array}$ 
3c, ${ }^{1} \mathrm{H}$ NMR $\left(500 \mathrm{MHz}, \mathrm{CDCl}_{3}\right)$

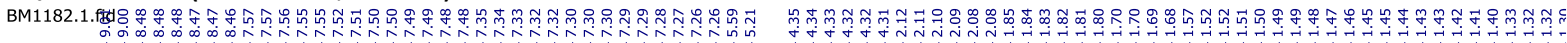
ن

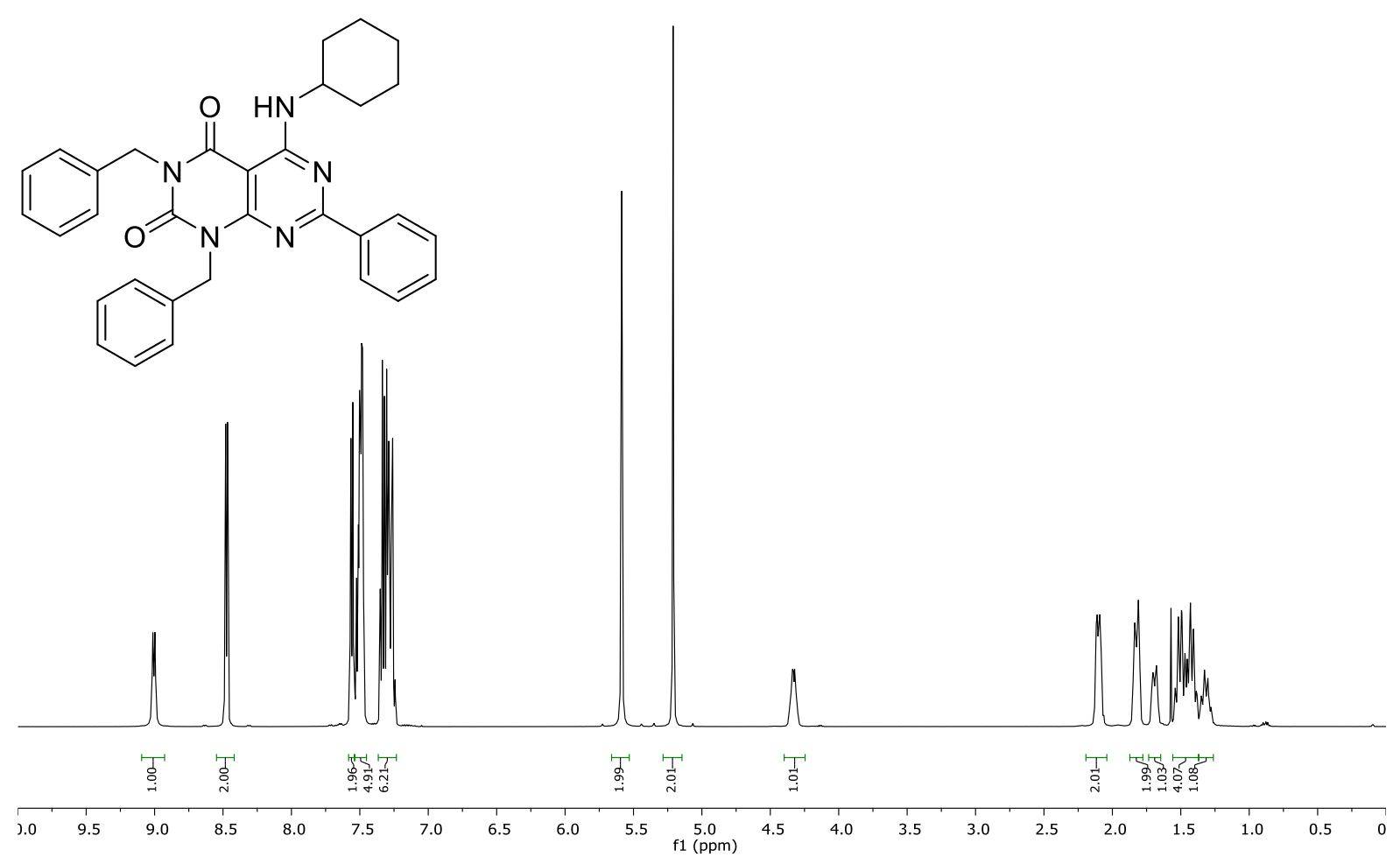

3c, ${ }^{1} \mathrm{H}$ NMR $\left(126 \mathrm{MHz}, \mathrm{CDCl}_{3}\right)$

BM1182.2.fid

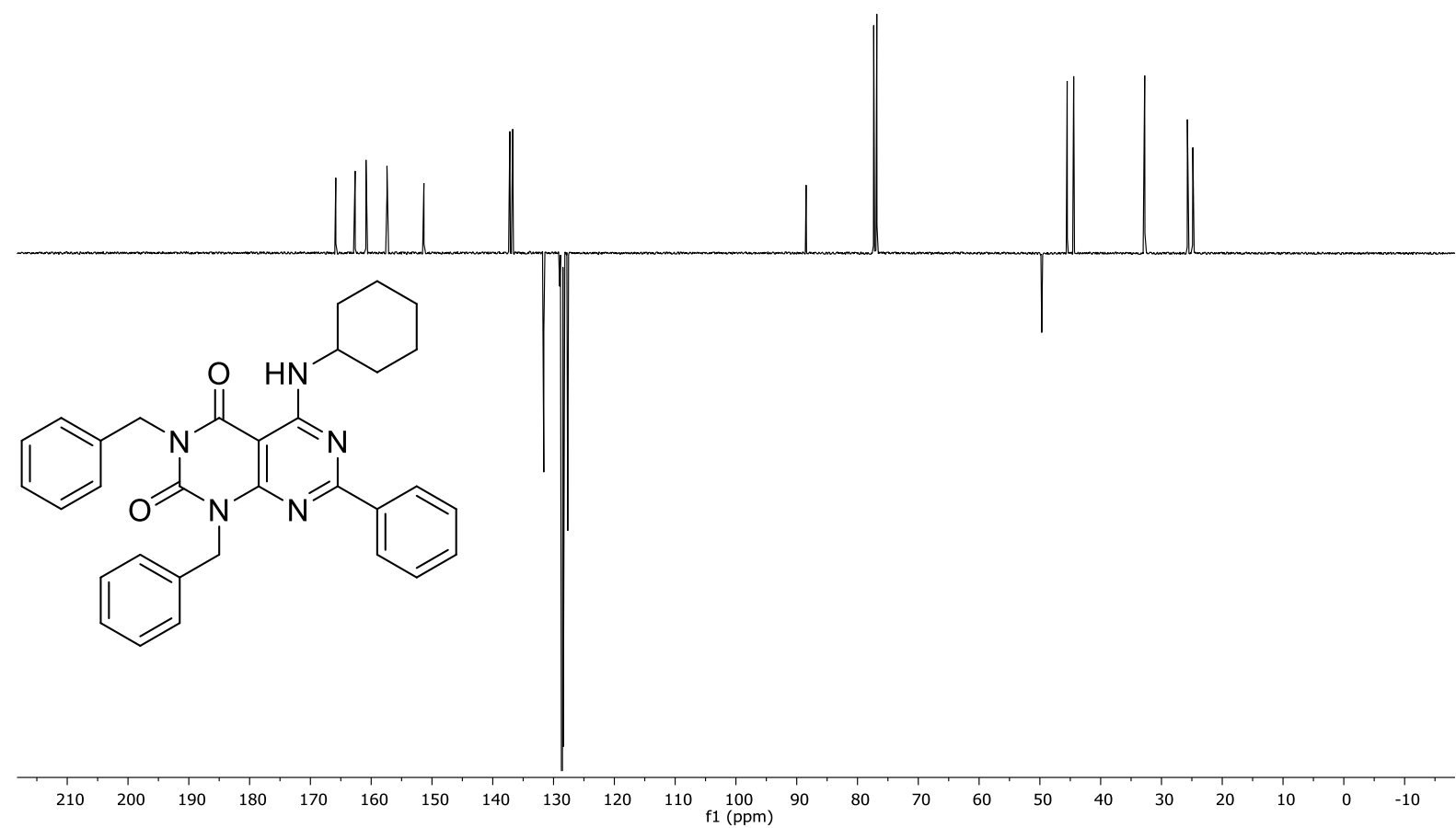


3d, ${ }^{1} \mathrm{H}$ NMR $\left(500 \mathrm{MHz}, \mathrm{CDCl}_{3}\right)$

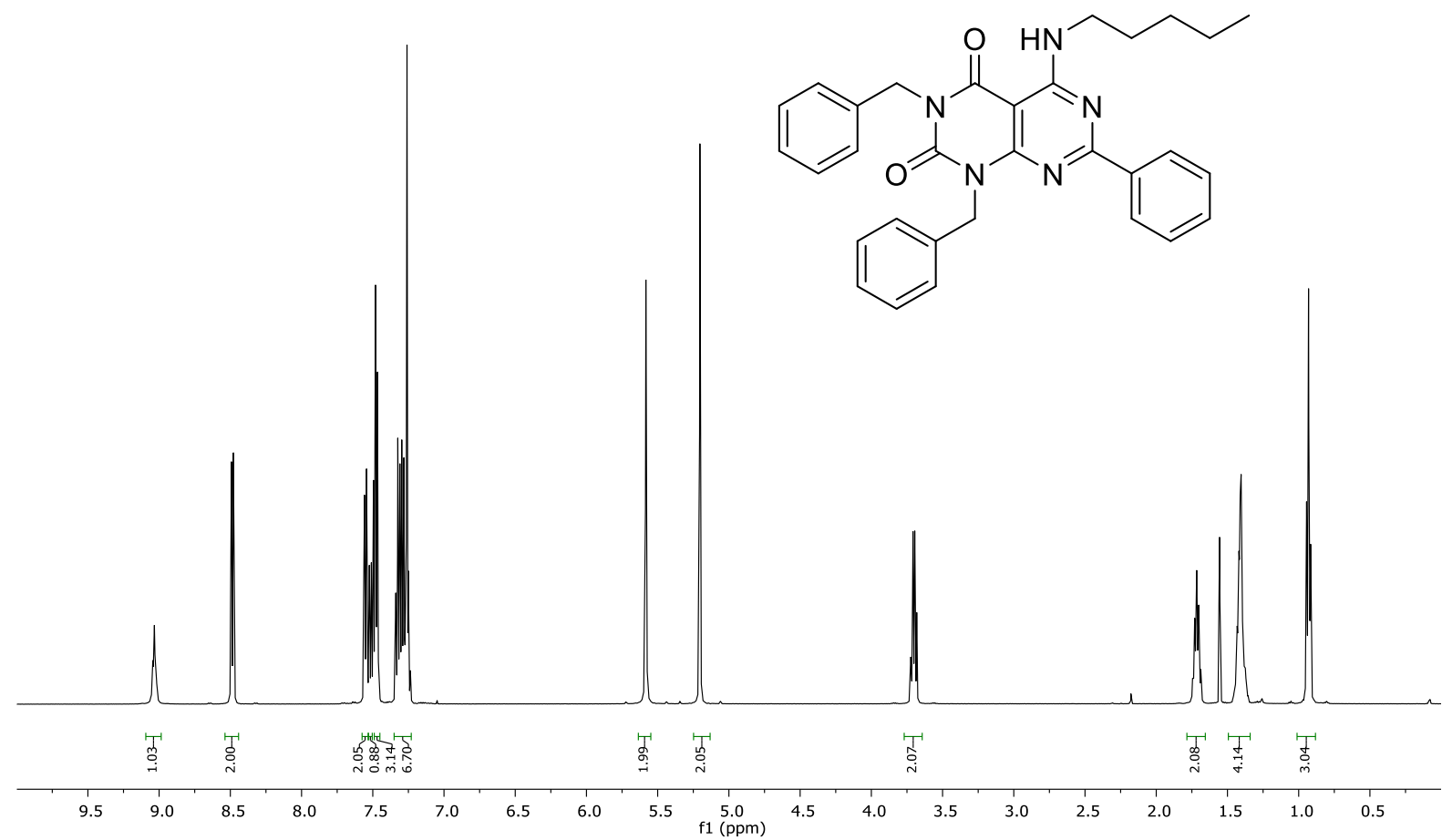

3d, ${ }^{1} \mathrm{H}$ NMR $\left(126 \mathrm{MHz}, \mathrm{CDCl}_{3}\right)$

BM1165.2.fid

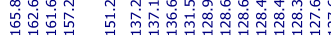
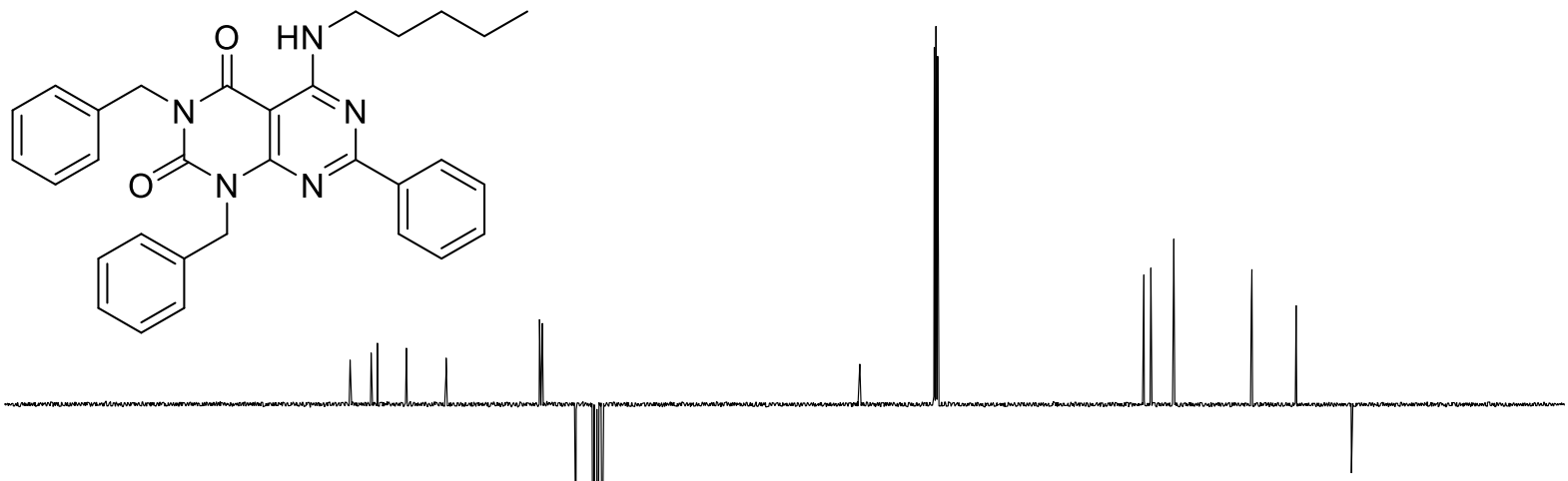

$\begin{array}{lllllllllllllllllllllll}210 & 200 & 190 & 180 & 170 & 160 & 150 & 140 & 130 & 120 & 110 & 100 & 90 & 80 & 70 & 60 & 50 & 40 & 30 & 20 & 10 & 0 & -10\end{array}$ 
3e, ${ }^{1} \mathrm{H}$ NMR $\left(500 \mathrm{MHz}, \mathrm{CDCl}_{3}\right)$

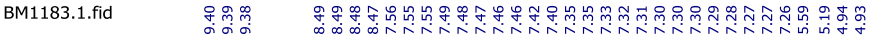

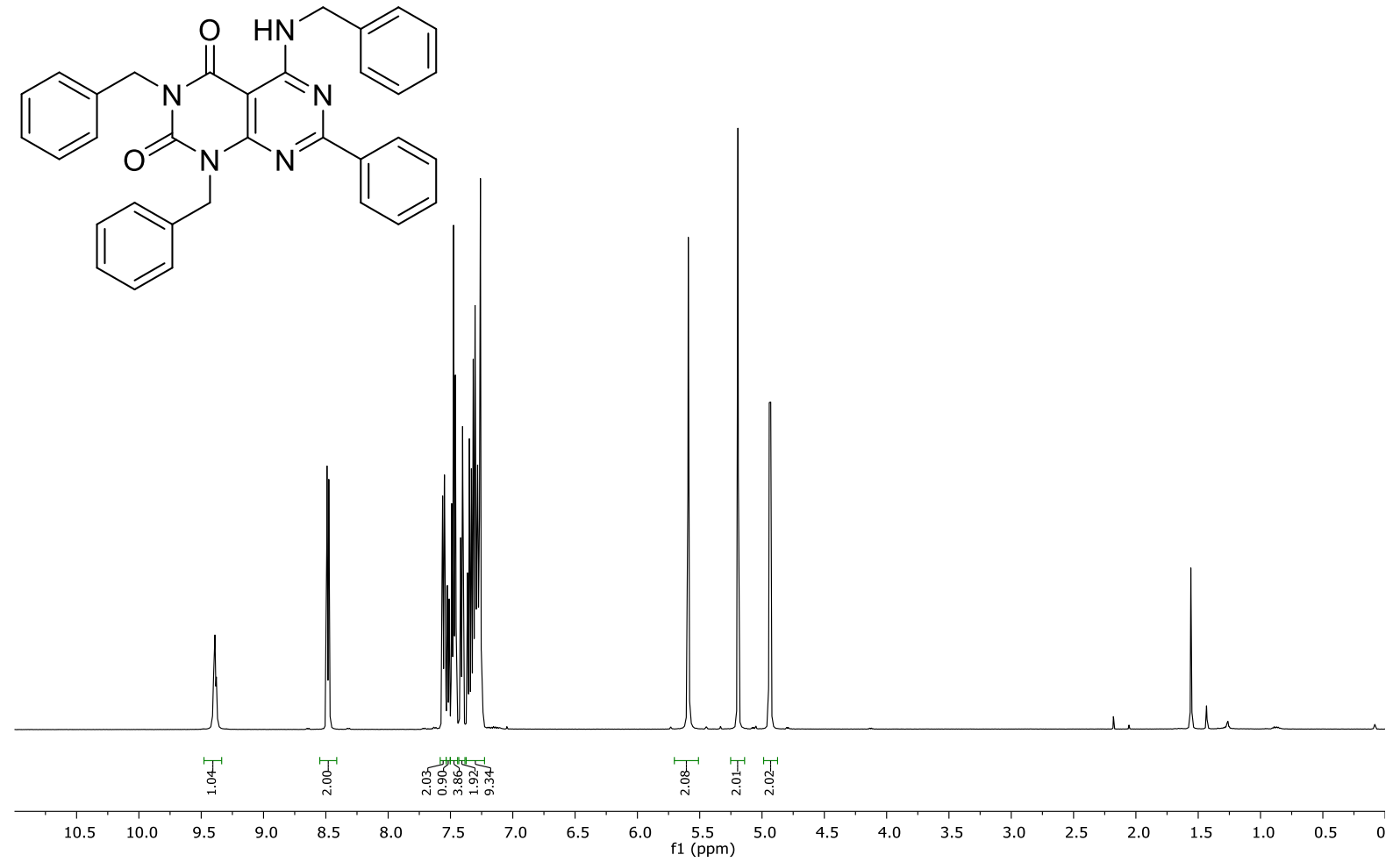

3e, ${ }^{1} \mathrm{H}$ NMR $\left(126 \mathrm{MHz}, \mathrm{CDCl}_{3}\right)$

BM1183.2.fid

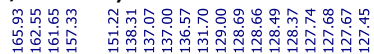

i)

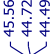

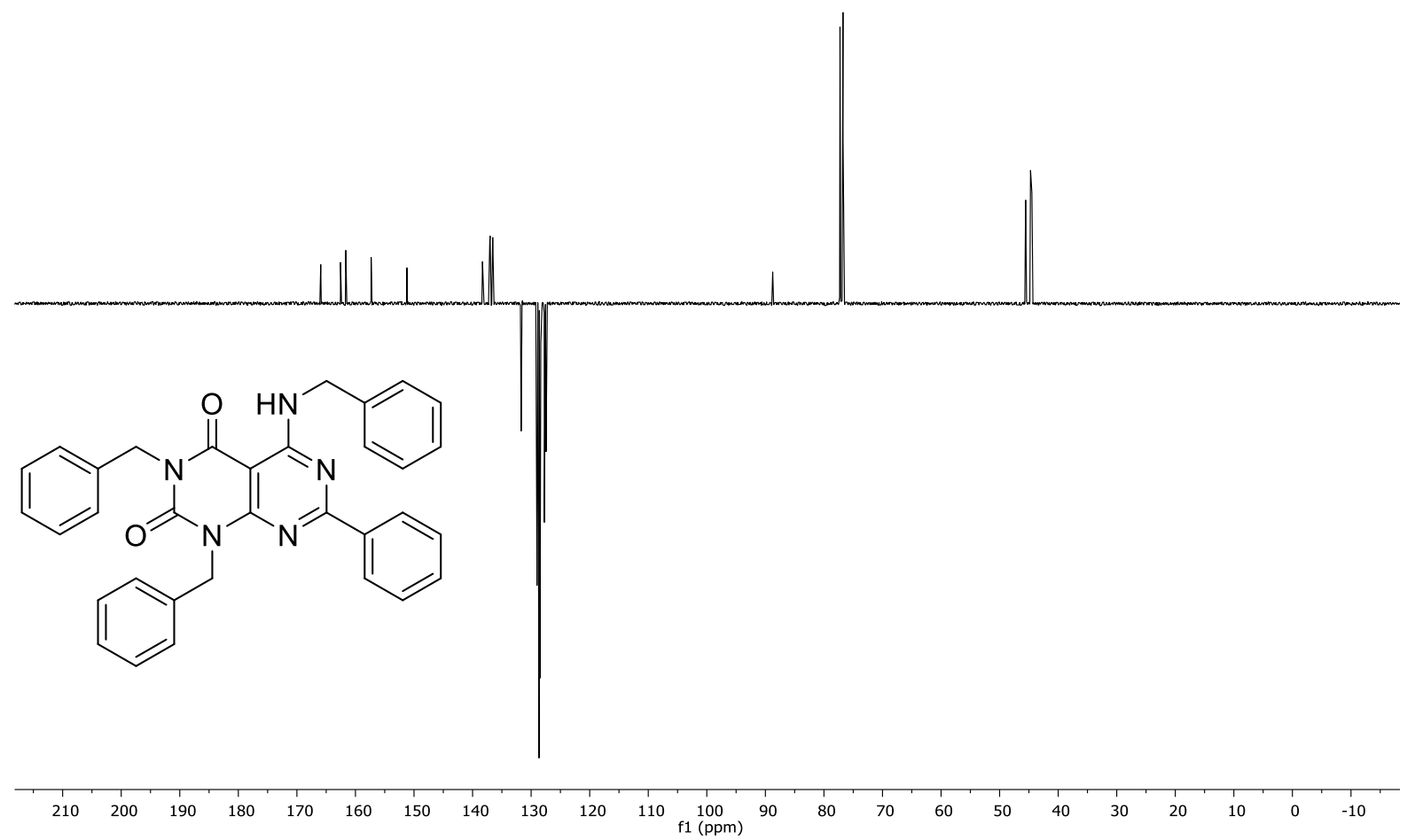


3f, ${ }^{1} \mathrm{H}$ NMR (500 MHz, $\mathrm{CDCl}_{3}$ )<smiles>CC(C)(C)CC(C)(C)Nc1nc(-c2ccccc2)nc2c1c(=O)n(Cc1ccccc1)c(=O)n2Cc1ccccc1</smiles>

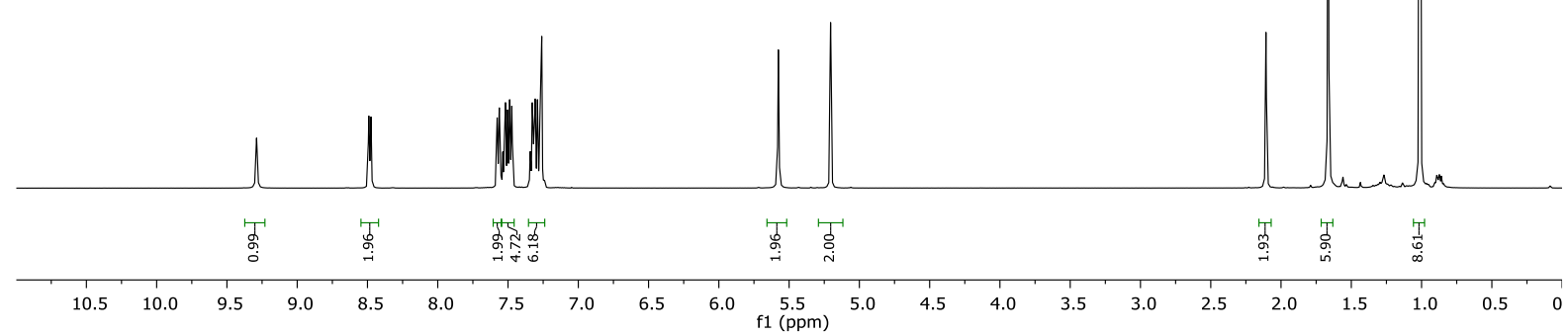

3f, ${ }^{1} \mathrm{H}$ NMR (126 MHz, $\mathrm{CDCl}_{3}$ )

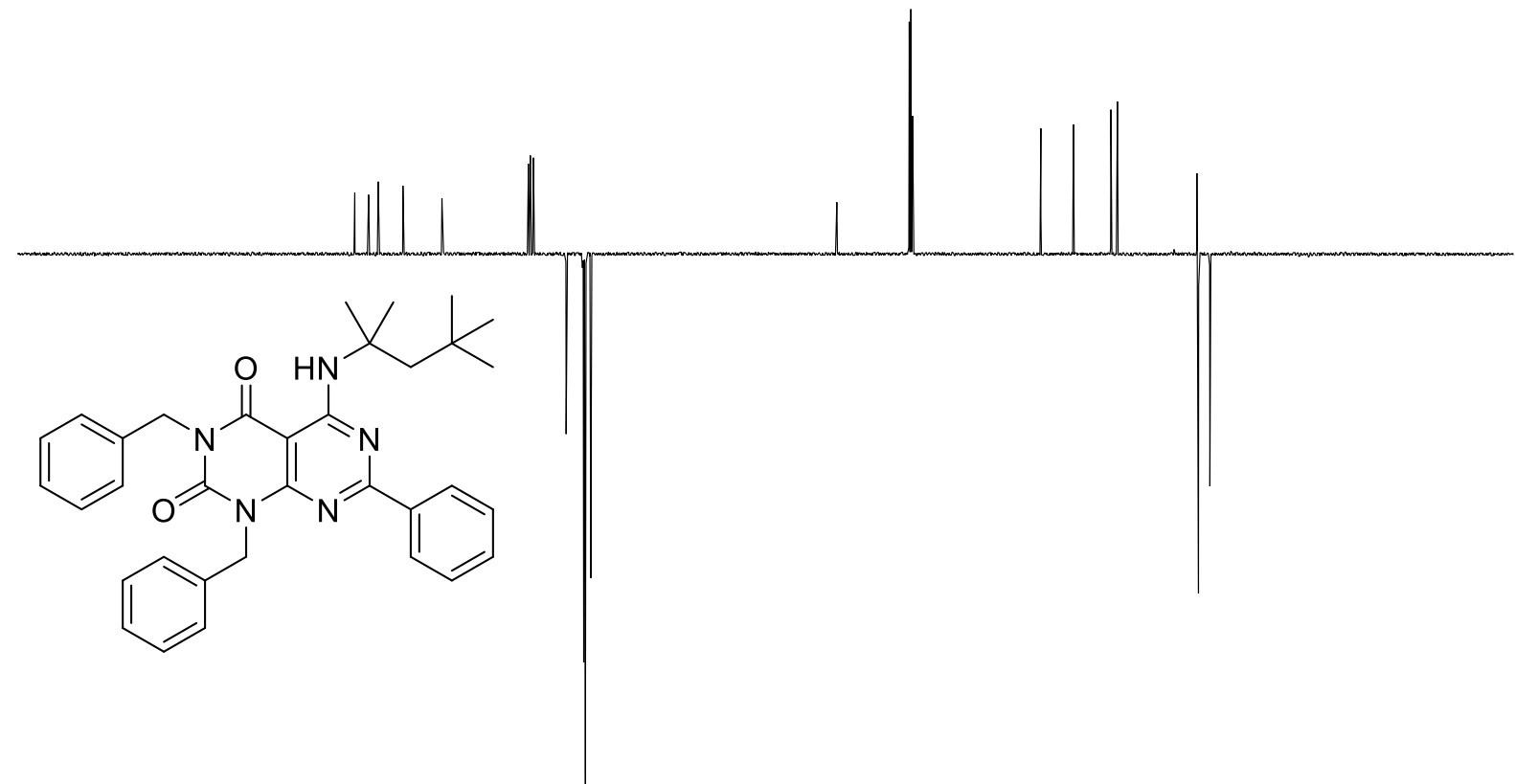

$\begin{array}{llllllllllllllllllllllllll}1 & 10 & 200 & 190 & 180 & 170 & 160 & 150 & 140 & 130 & 120 & 110 & 100 & 90 & 80 & 70 & 60 & 50 & 40 & 30 & 20 & 10 & 0 & -10\end{array}$ 
<smiles>CCN(CC)CCCC(C)Nc1nc(-c2ccccc2)nc2c1c(=O)n(Cc1ccccc1)c(=O)n2Cc1ccccc1</smiles>

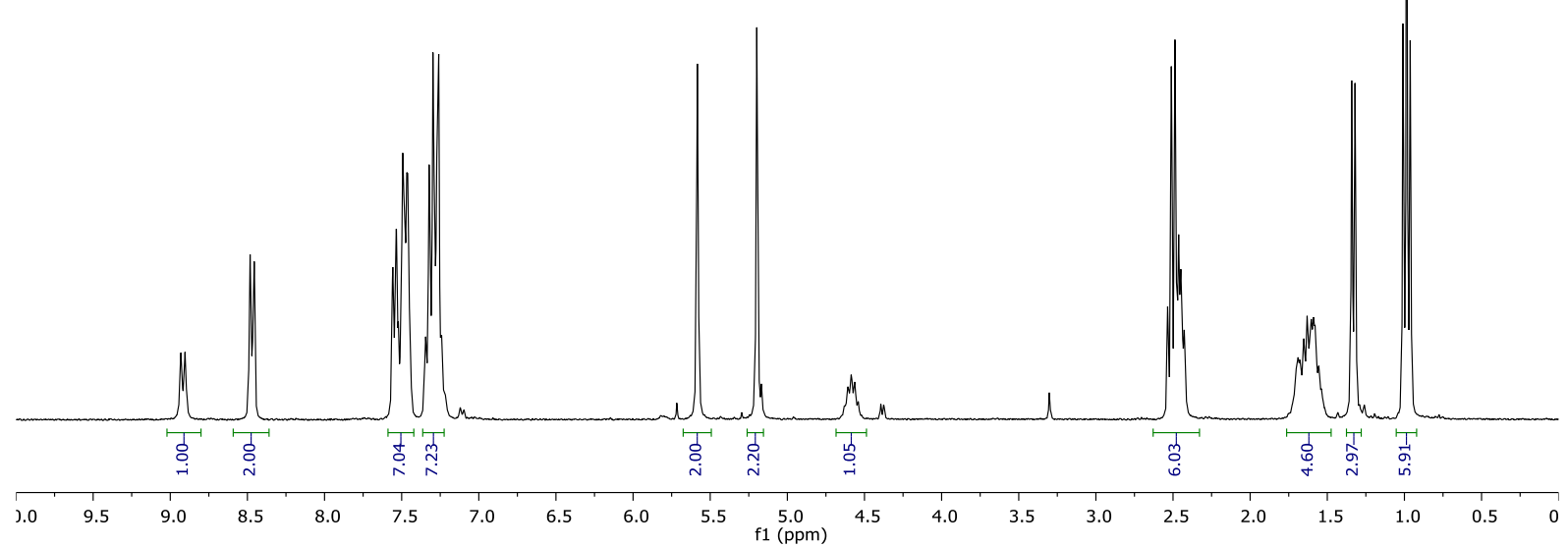

3g, ${ }^{1} \mathrm{H}$ NMR $\left(300 \mathrm{MHz}, \mathrm{CDCl}_{3}\right),(\mathrm{JC782A})$

JC782A.11.fid

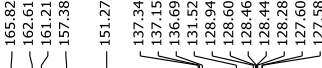

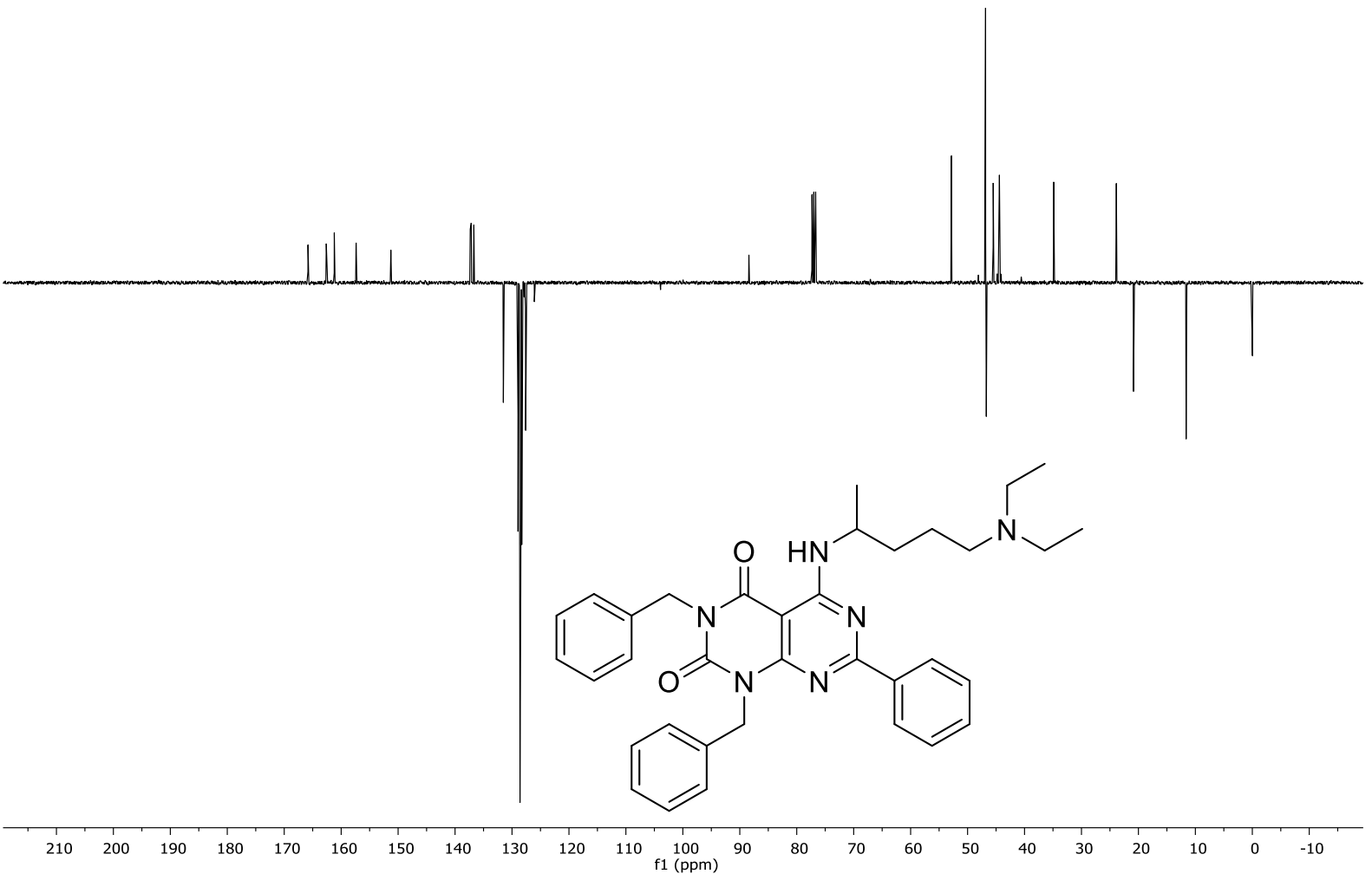


3h, ${ }^{1} \mathrm{H}$ NMR (500 MHz, $\mathrm{CDCl}_{3}$ ) BM1172.1.fi

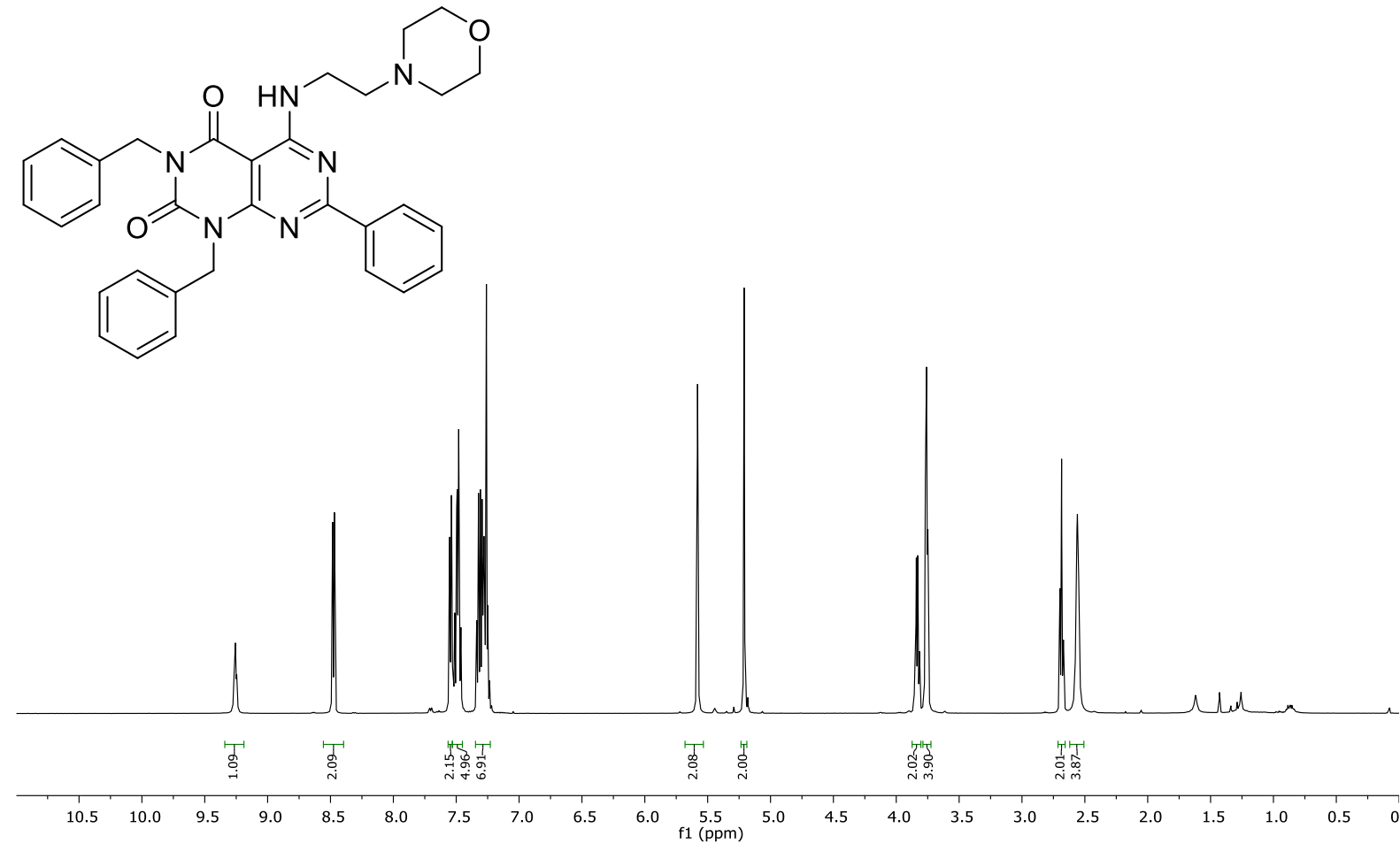

3h, ${ }^{1} \mathrm{H}$ NMR (126 MHz, $\mathrm{CDCl}_{3}$ )

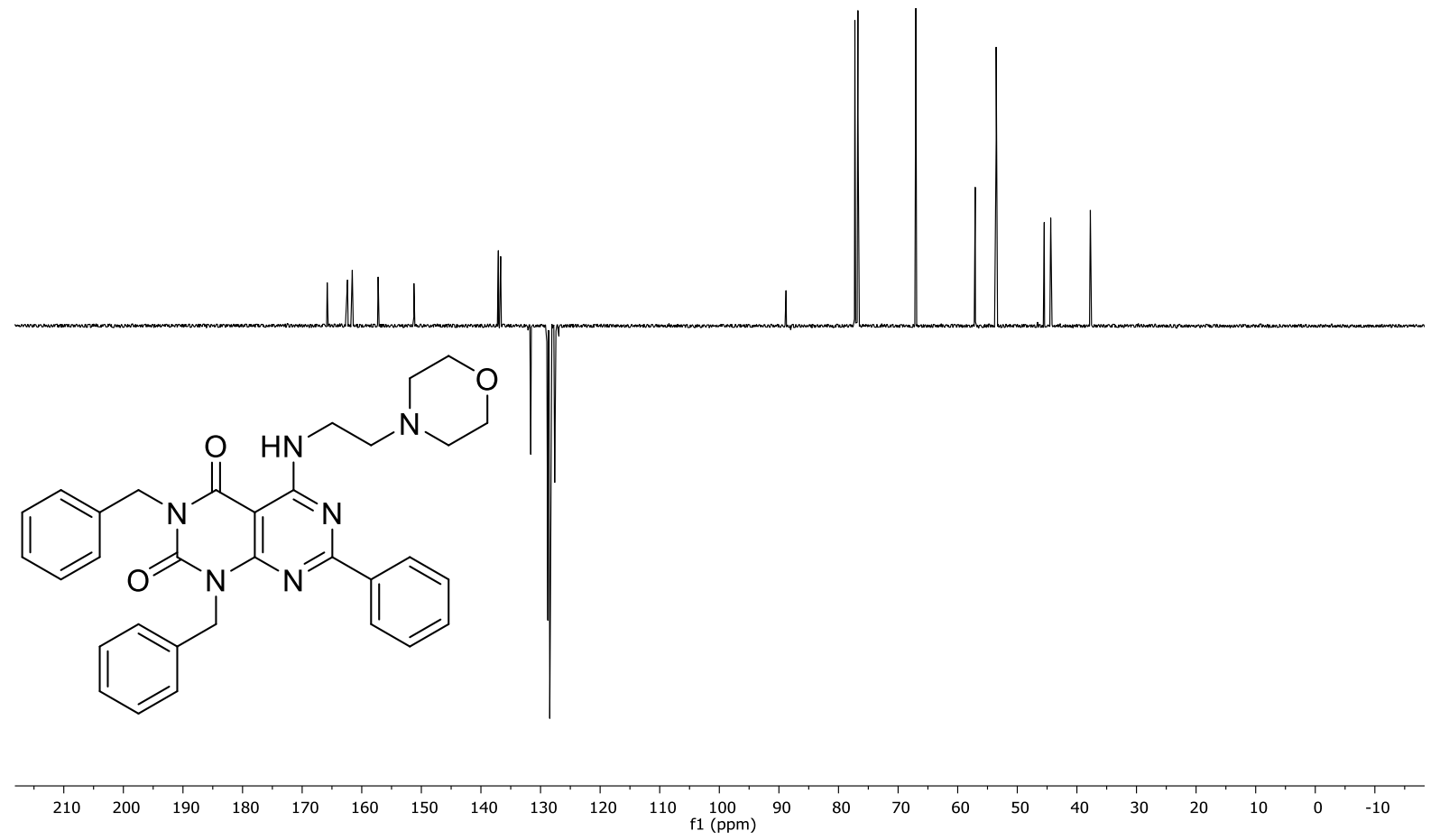


3i, ${ }^{1} \mathrm{H}$ NMR (300 MHz, $\left.\mathrm{CDCl}_{3}\right)$, (JC784A)

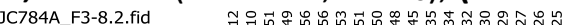

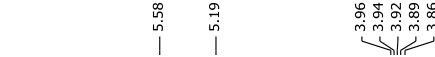<smiles>COc1ccc(CCNc2nc(-c3ccccc3)nc3c2c(=O)n(Cc2ccccc2)c(=O)n3Cc2ccccc2)cc1OC</smiles>

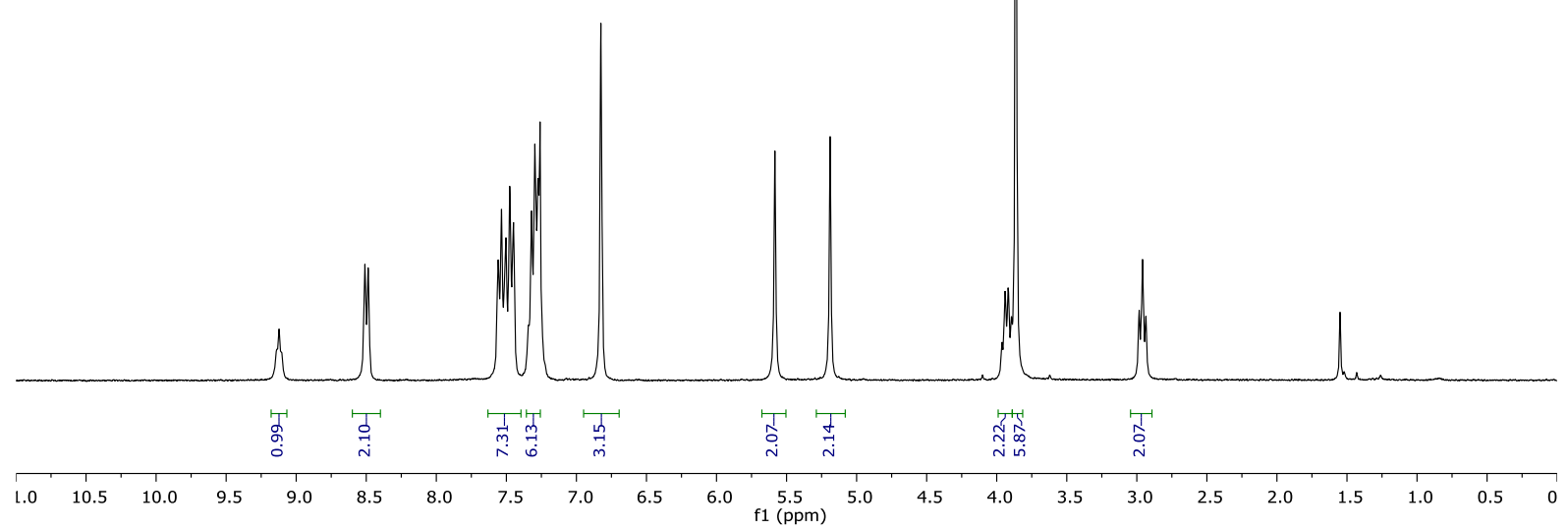

$3 \mathbf{i},{ }^{13} \mathrm{C} \mathrm{NMR}\left(100 \mathrm{MHz}, \mathrm{CDCl}_{3}\right),(\mathrm{JC784A})$

JC784A.11.fid

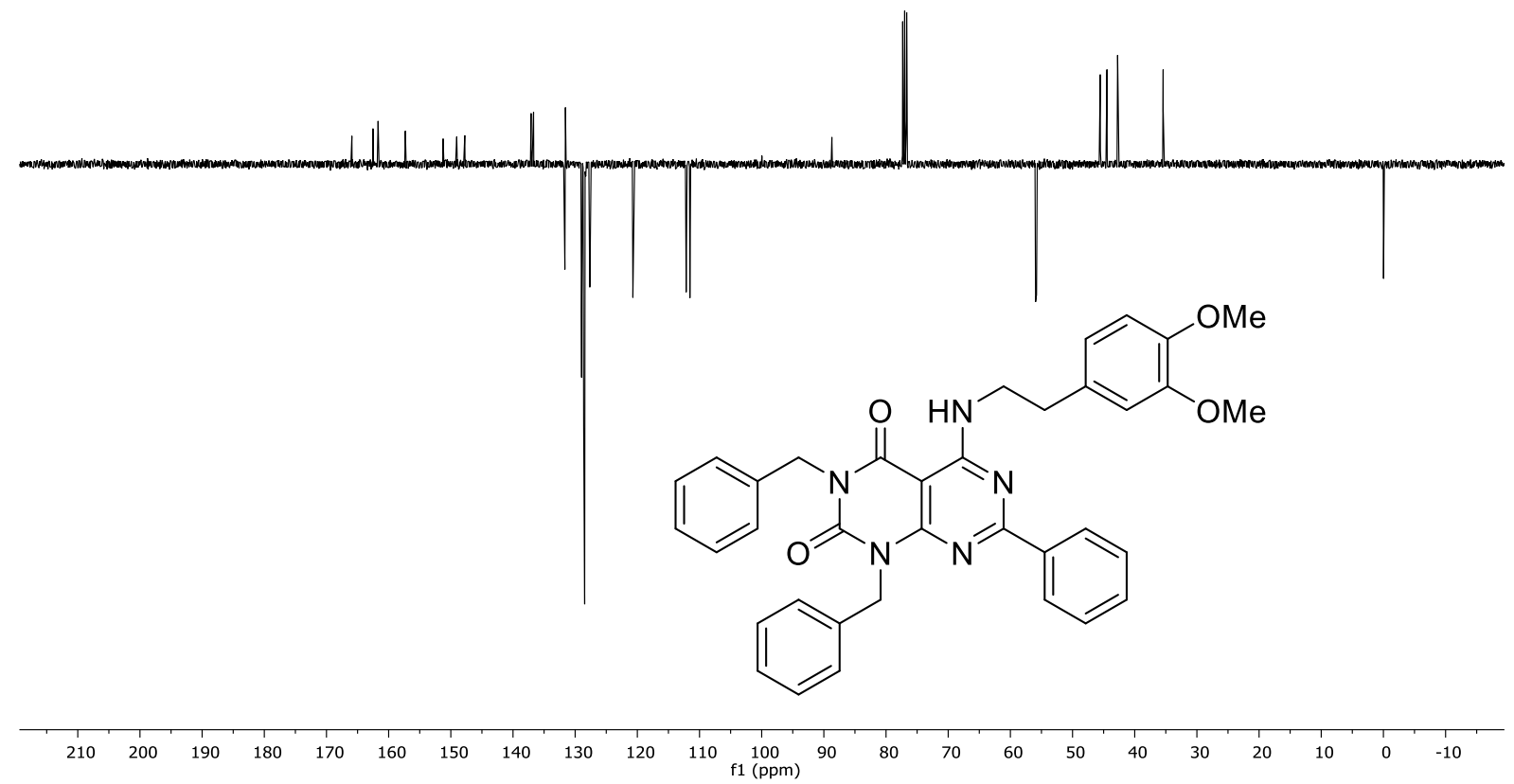


3j, ${ }^{1} \mathrm{H}$ NMR (300 MHz, $\left.\mathrm{CDCl}_{3}\right)$ (HL064)

HL064.2.fid :

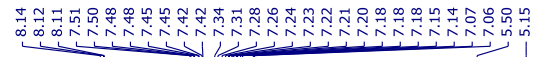<smiles>Cc1cccc(C)c1Nc1nc(-c2ccccc2)nc2c1c(=O)n(Cc1ccccc1)c(=O)n2Cc1ccccc1</smiles>

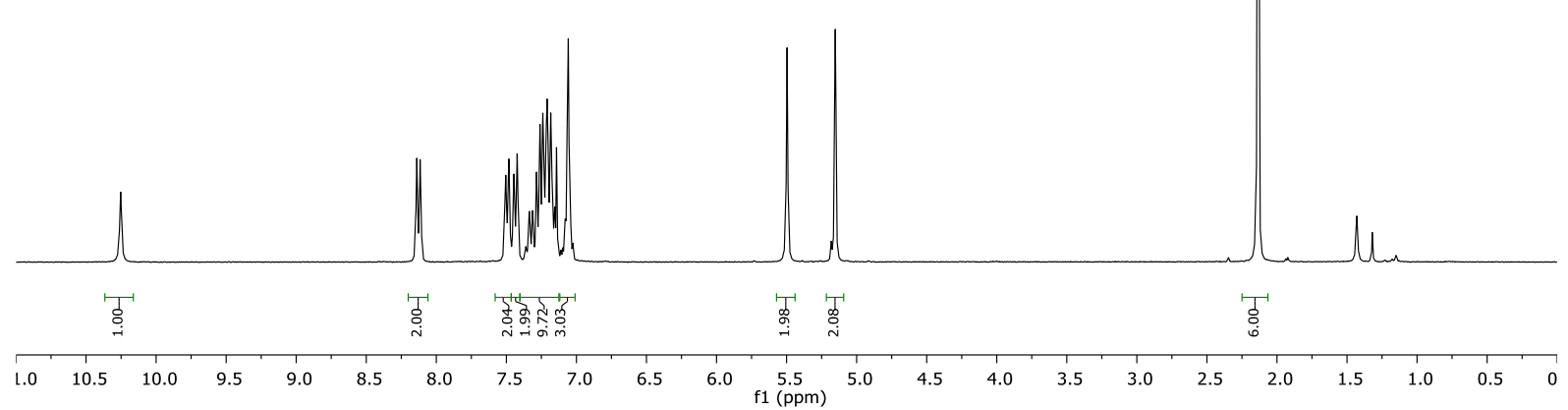

3j, ${ }^{1} \mathrm{H}$ NMR (100 MHz, CDCl $)$ (HL064/BM1160)

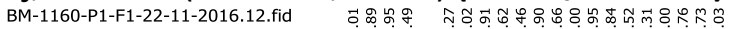

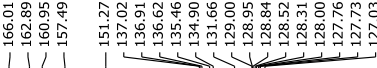

|

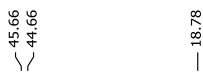

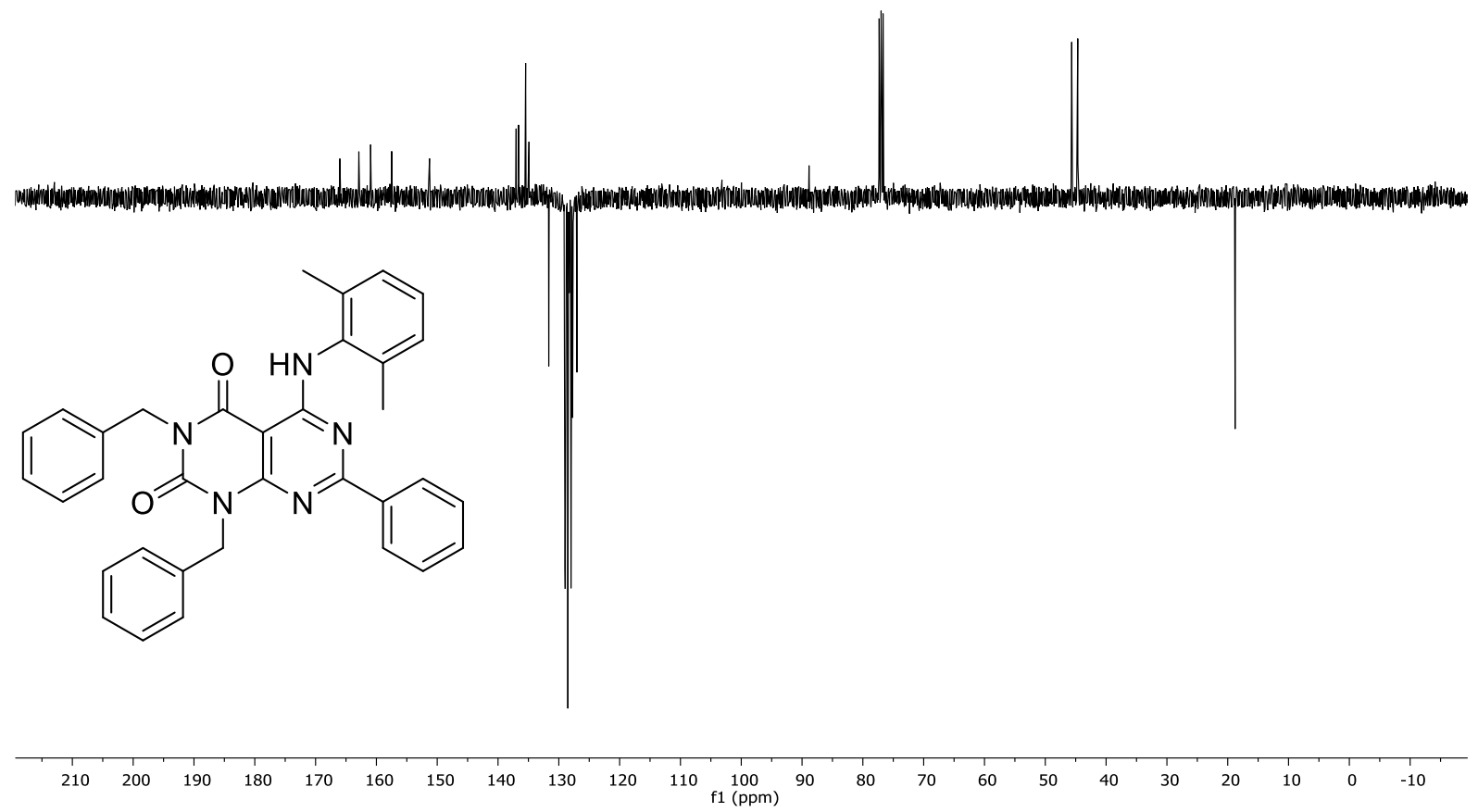


3m, ${ }^{1} \mathrm{H} \mathrm{NMR} \mathrm{(300} \mathrm{MHz,} \mathrm{CDCl}_{3}$ ) (JC803B)<smiles>COC(=O)c1ccccc1Nc1nc(-c2ccccc2)nc2c1c(=O)n(Cc1ccccc1)c(=O)n2Cc1ccccc1</smiles>
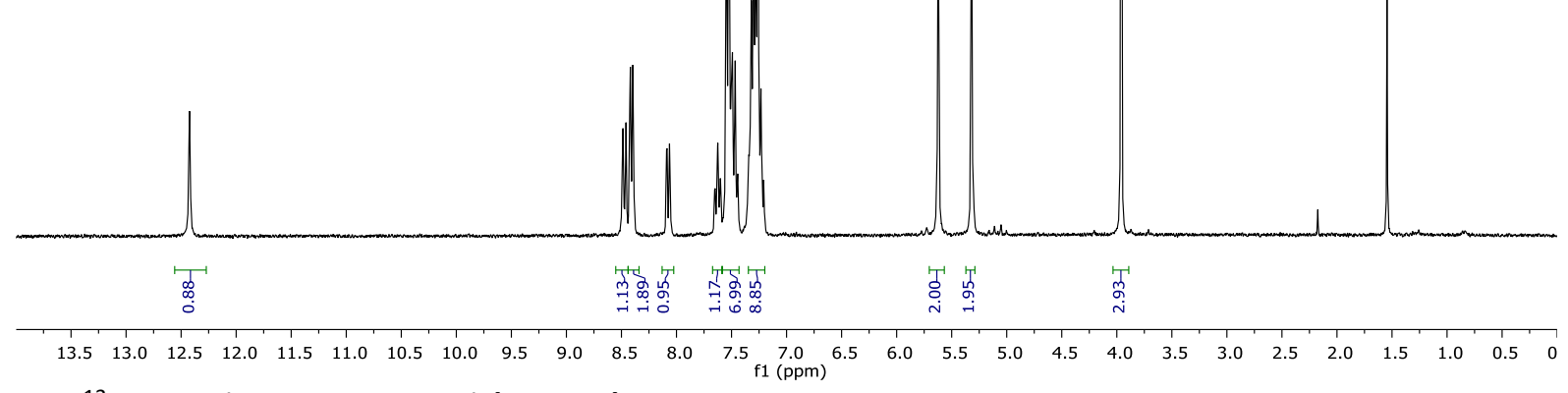

$3 m,{ }^{13} \mathrm{C} \mathrm{NMR} \mathrm{(126} \mathrm{MHz,} \mathrm{CDCl} 3$ ) (JC803B)

JC958_pp.3.fid

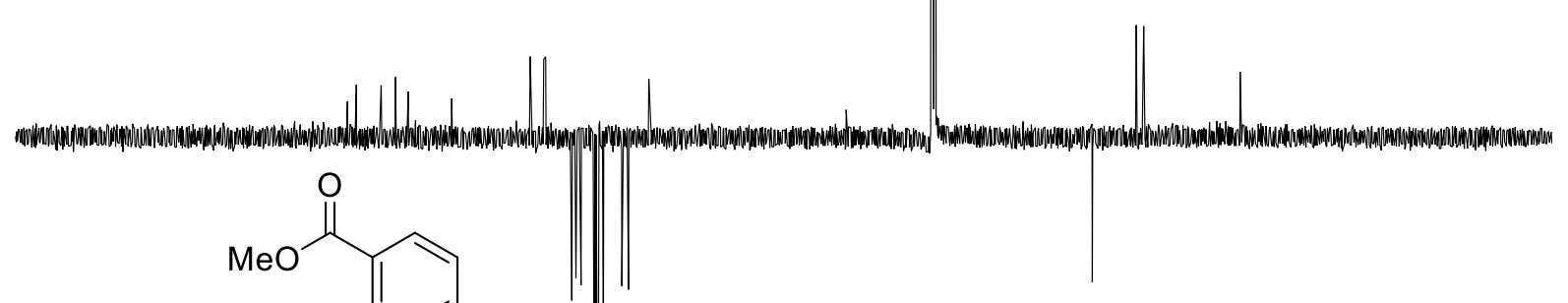<smiles>C/C=C\C(=O)Nc1nc(-c2ccccc2)nc2c1c(=O)n(Cc1ccccc1)c(=O)n2Cc1ccccc1</smiles>

$\begin{array}{llllllllllllllllllllllllllllll}210 & 200 & 190 & 180 & 170 & 160 & 150 & 140 & 130 & 120 & 110 & 100 & 90 & 80 & 70 & 60 & 50 & 40 & 30 & 20 & 10 & 0 & -10\end{array}$ 
4a, ${ }^{1} \mathrm{H}$ NMR (300 MHz, $\mathrm{CDCl}_{3}$ )

HL116.3.fid<smiles>CC(C)(C)Nc1nc(-c2ccc(Cl)cc2)nc2c1c(=O)n(Cc1ccccc1)c(=O)n2Cc1ccccc1</smiles>

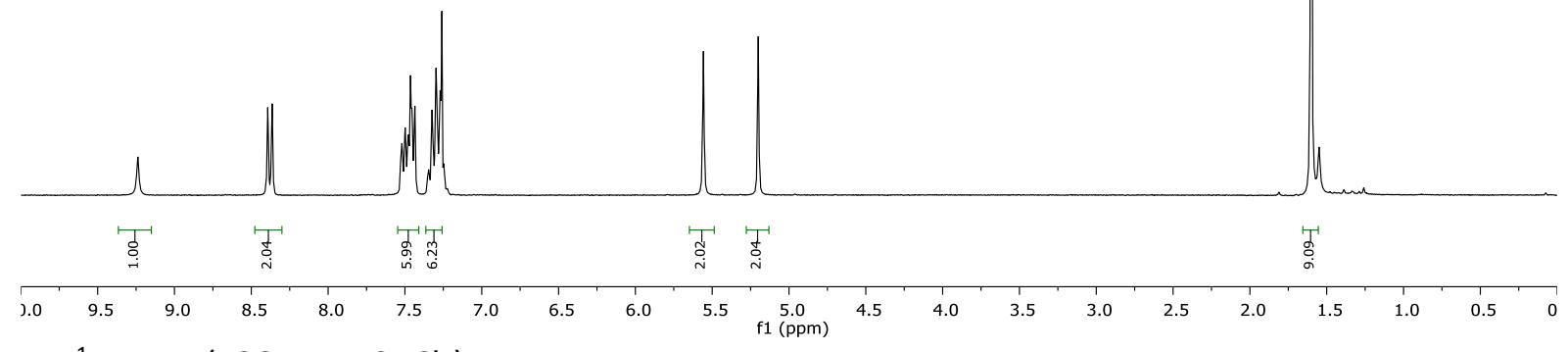

4a, ${ }^{1} \mathrm{H}$ NMR (126 MHz, $\mathrm{CDCl}_{3}$ )

HL116.2.fid

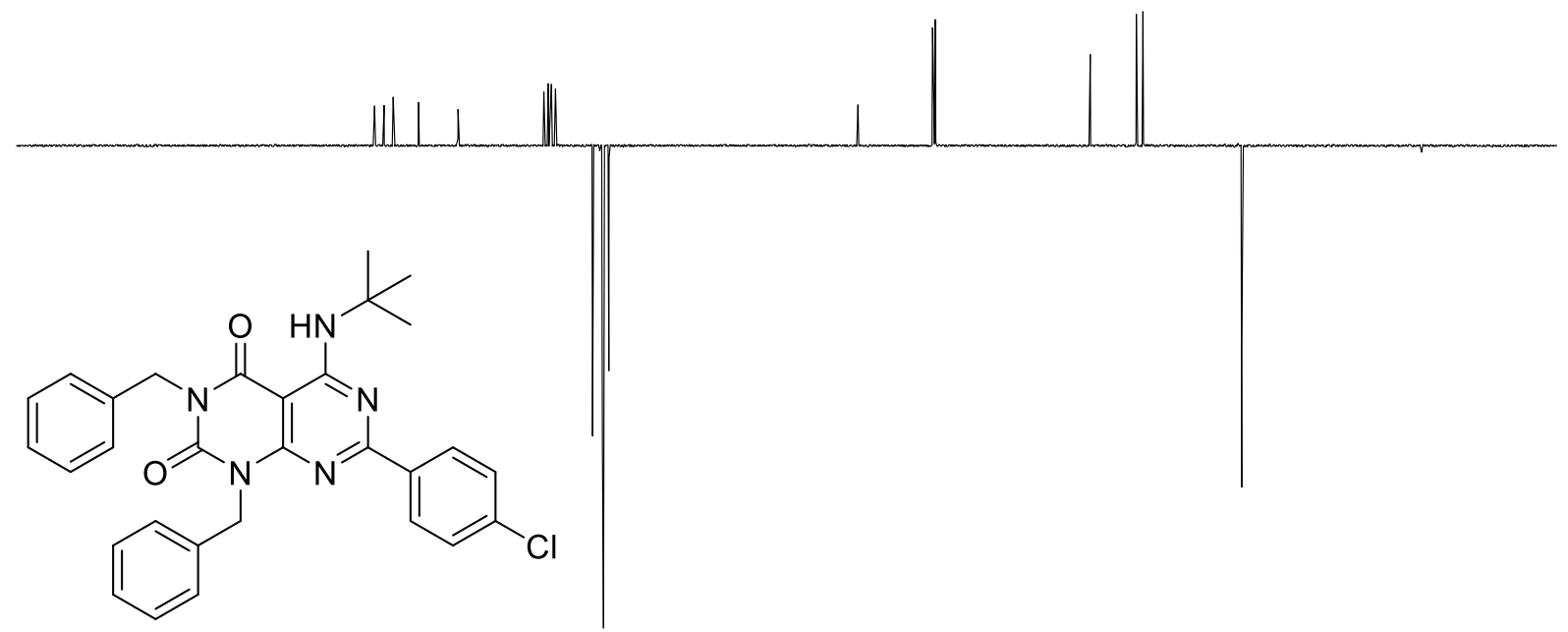

$\begin{array}{llllllllllllllllllllllllll}210 & 200 & 190 & 180 & 170 & 160 & 150 & 140 & 130 & 120 & 110 & 100 & 90 & 80 & 70 & 60 & 50 & 40 & 30 & 20 & 10 & 0 & -10 & -:\end{array}$ 
4b, ${ }^{1} \mathrm{H}$ NMR (300 MHz, $\mathrm{CDCl}_{3}$ )

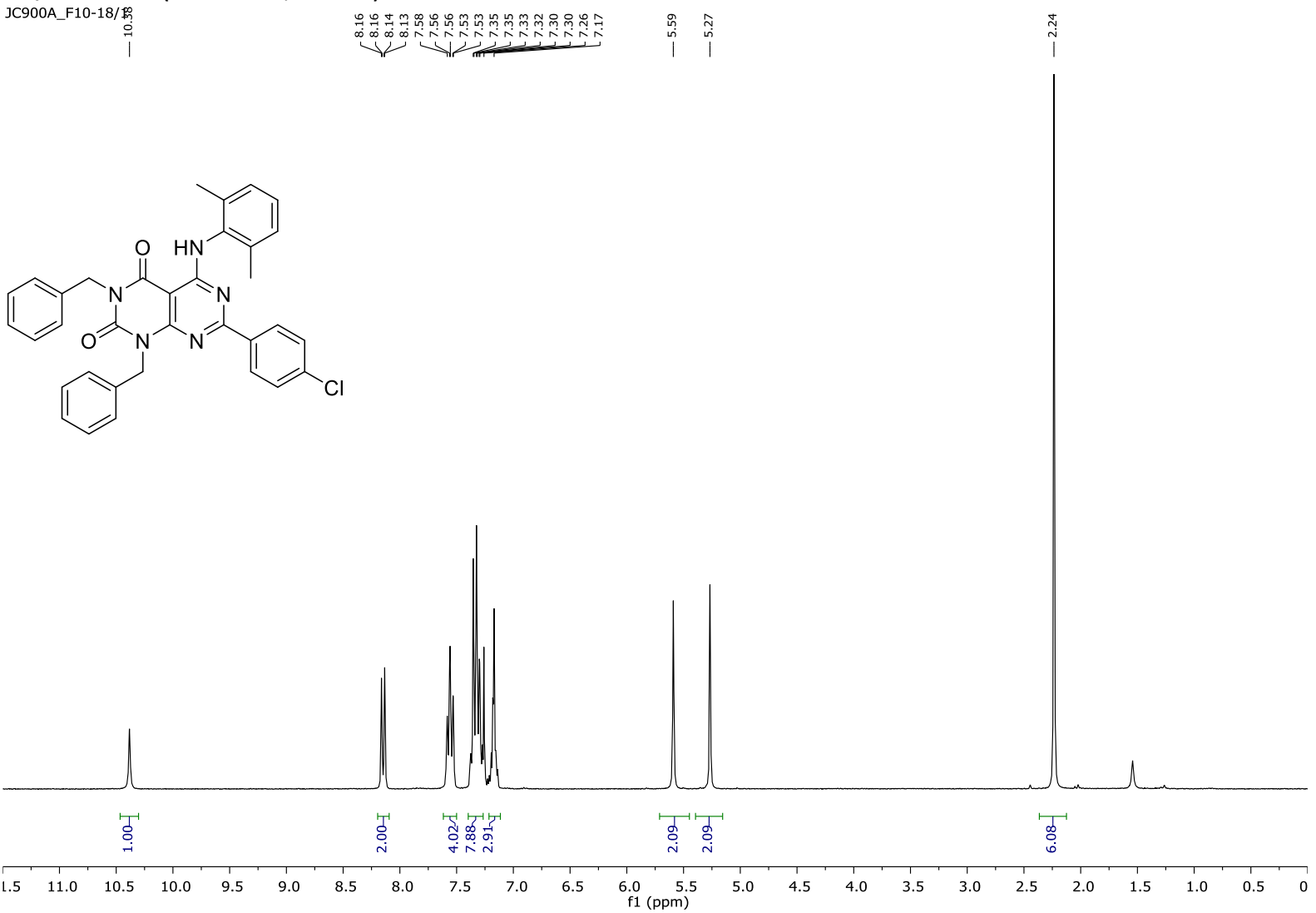

4b, ${ }^{13} \mathrm{C}$ NMR (100 MHz, $\left.\mathrm{CDCl}_{3}\right)$

JC900A.11.fid

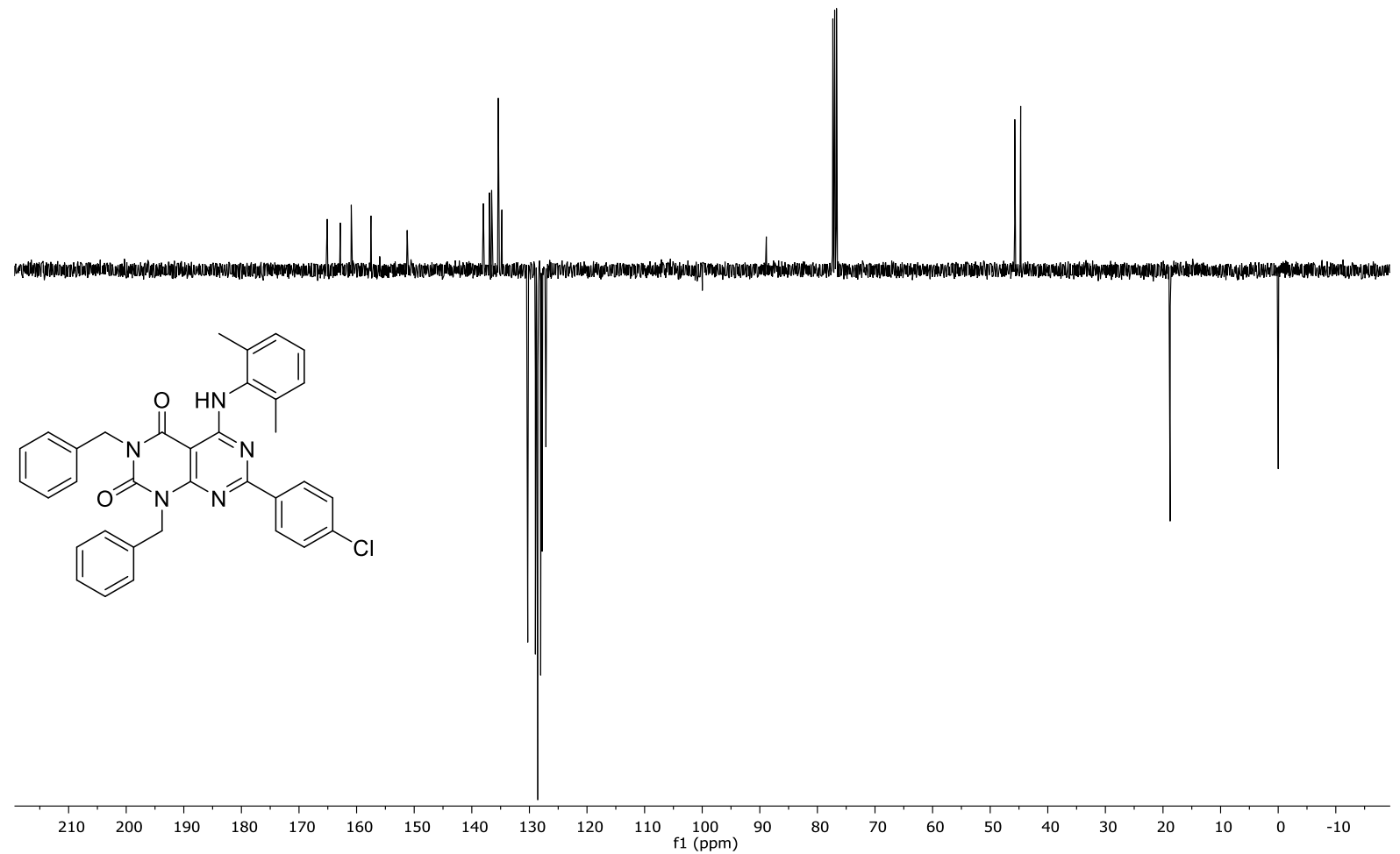


4c, ${ }^{1} \mathrm{H} \mathrm{NMR}\left(400 \mathrm{MHz}, \mathrm{CDCl}_{3}\right.$ ) (JC800B)

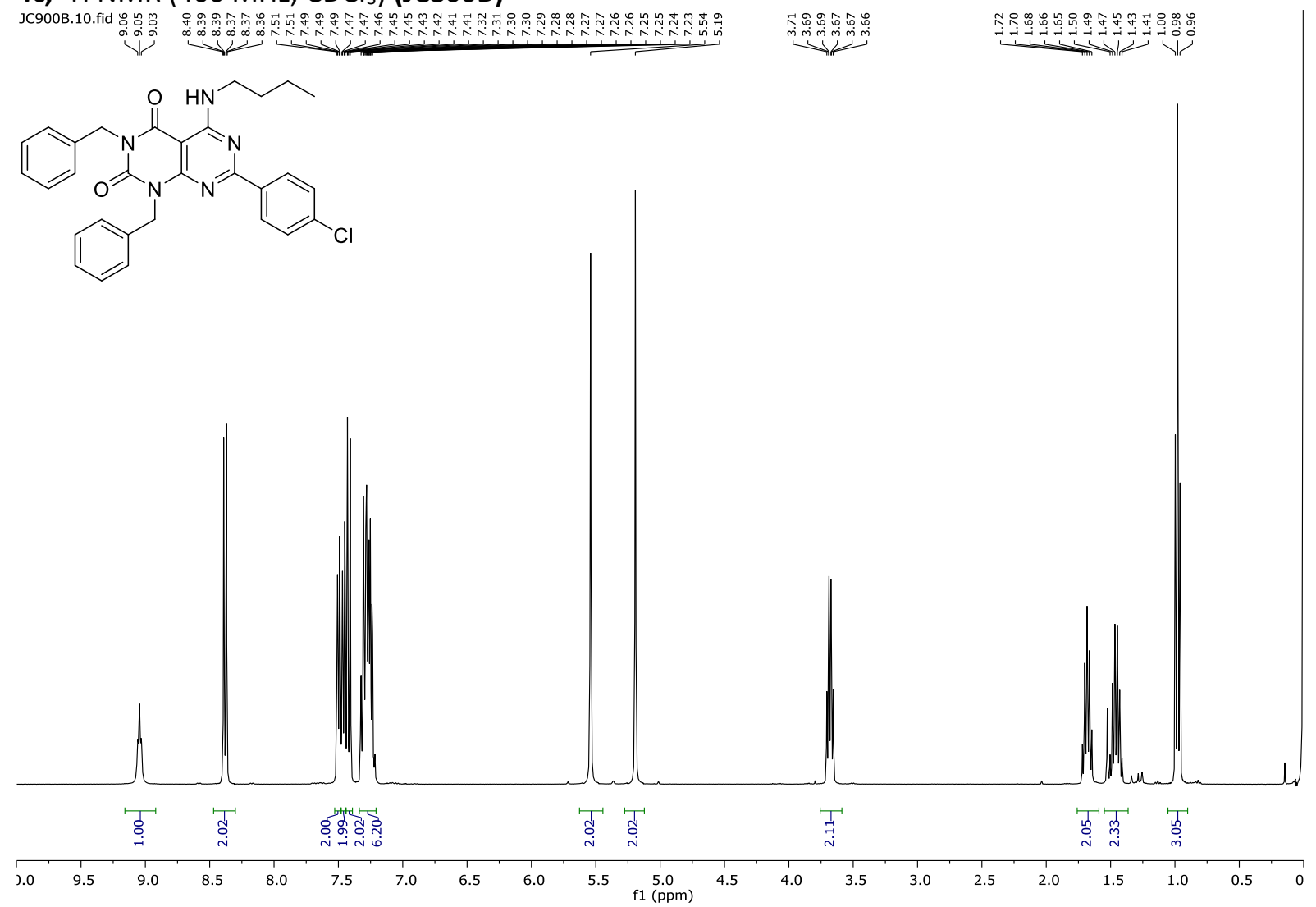

4c, ${ }^{13} \mathrm{C}$ NMR (100 MHz, CDCl $)$ (JC800B)

JC900B.11.fid

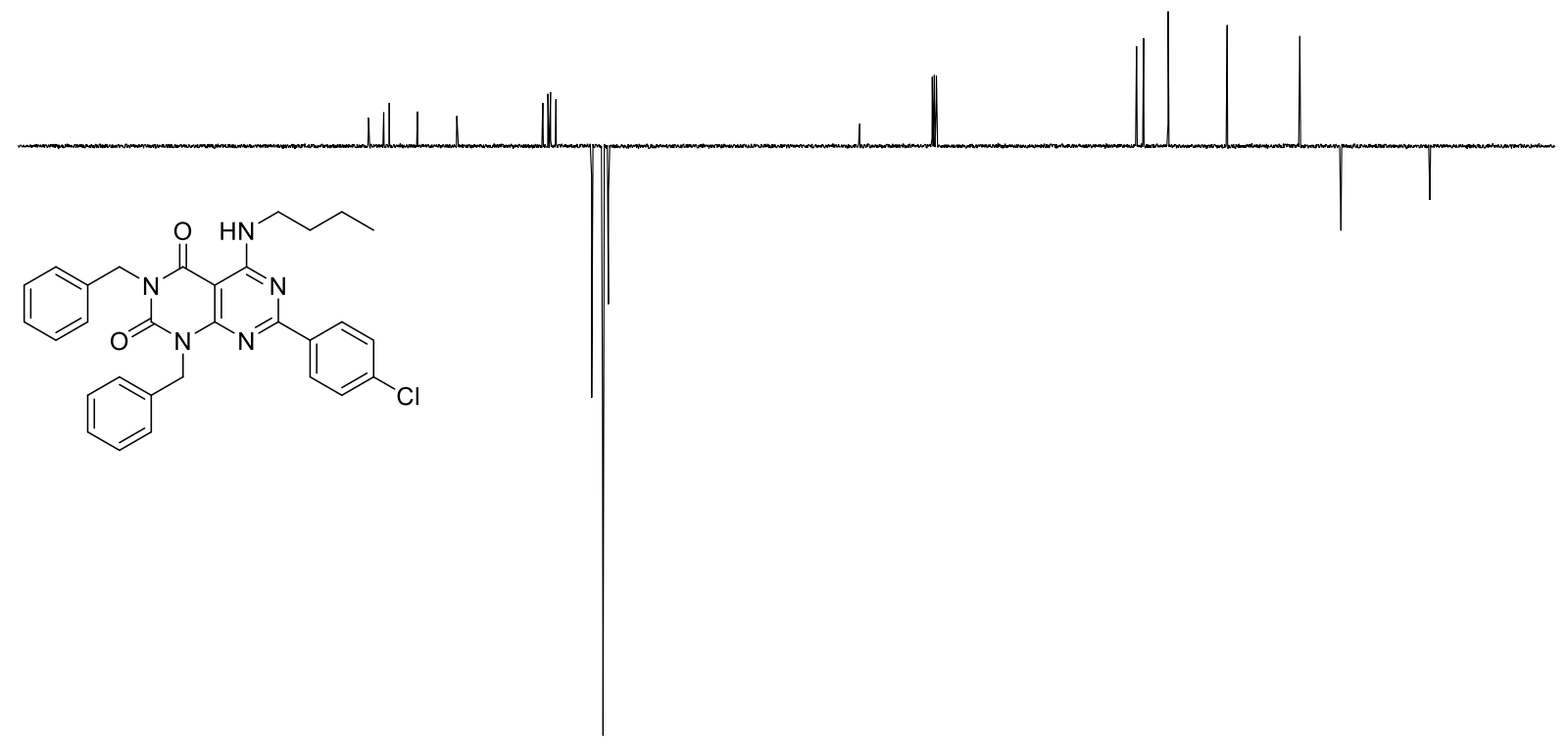

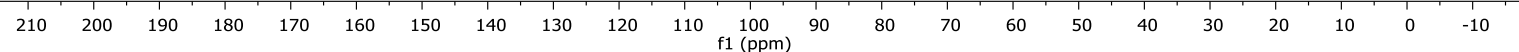


4d, ${ }^{1} \mathrm{H}$ NMR (300 MHz, $\mathrm{CDCl}_{3}$ )

HLo55/7 每<smiles>CC(C)(C)Nc1nc(-c2ccc(C(F)(F)F)cc2)nc2c1c(=O)n(Cc1ccccc1)c(=O)n2Cc1ccccc1</smiles>

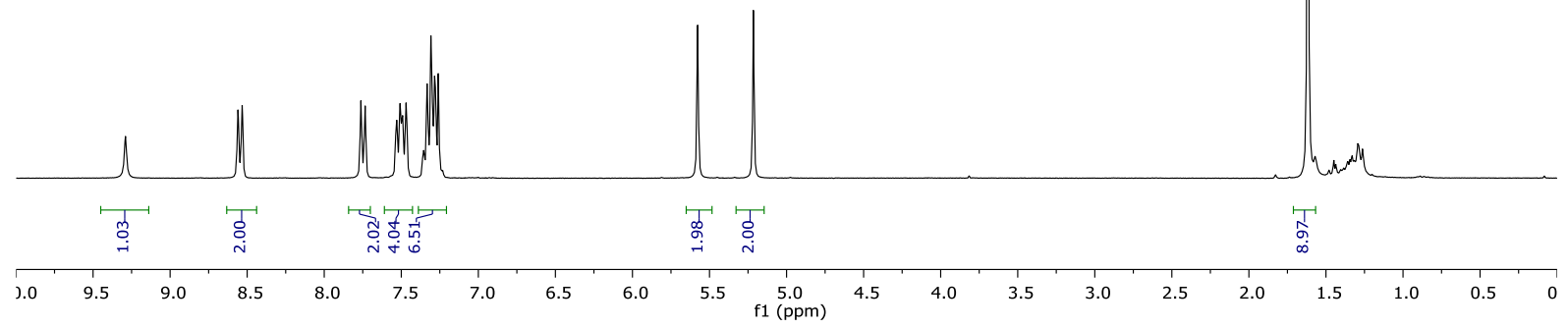

4d, ${ }^{1} \mathrm{H}$ NMR (126 MHz, $\mathrm{CDCl}_{3}$ )

HL055.2.fid

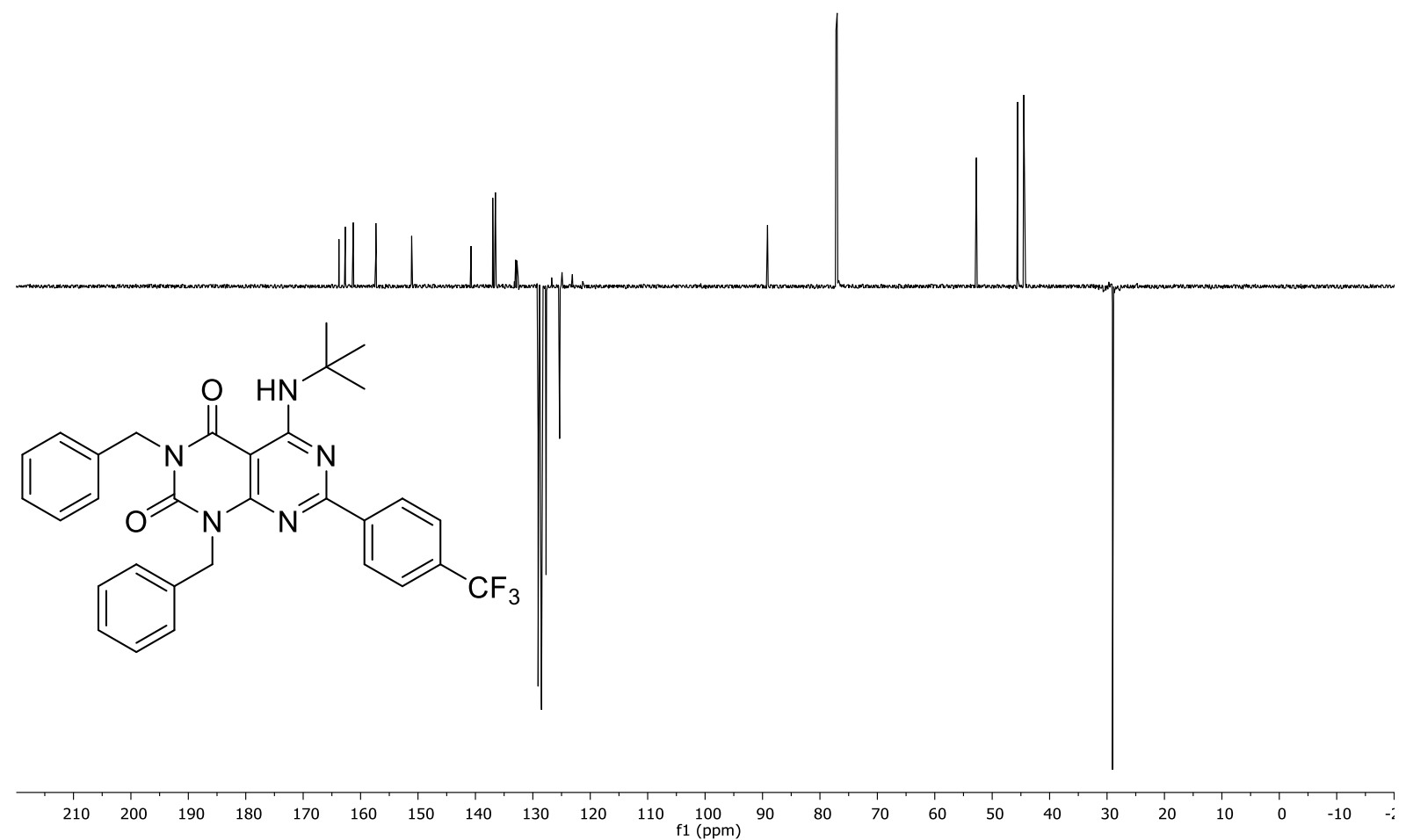


4e, ${ }^{1} \mathrm{H}$ NMR (300 MHz, $\mathrm{CDCl}_{3}$ )

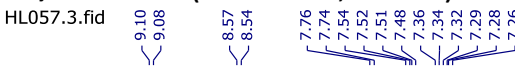

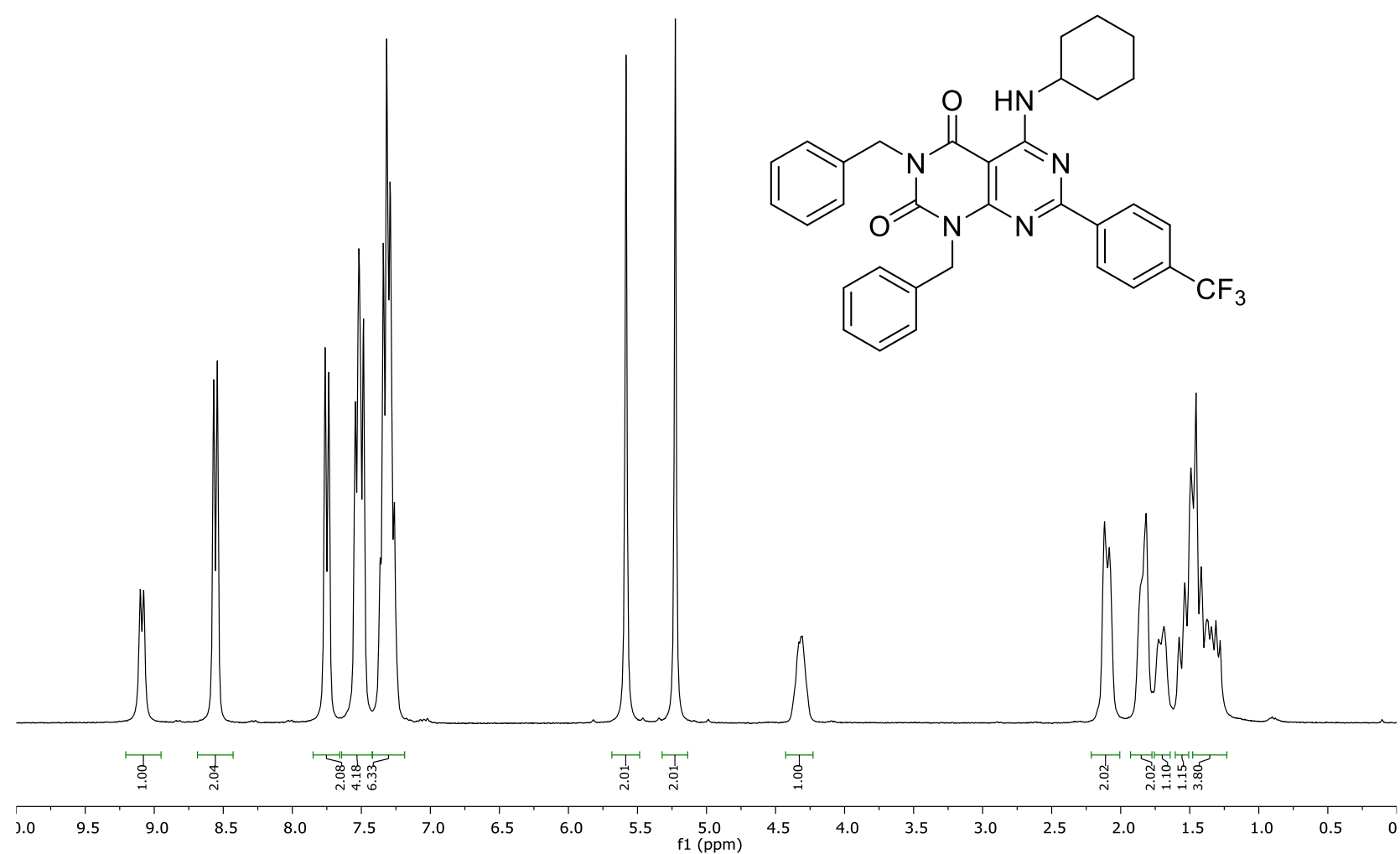

4e, ${ }^{1} \mathrm{H}$ NMR (126 MHz, $\mathrm{CDCl}_{3}$ )

HL057.1.fid

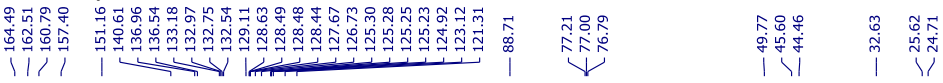

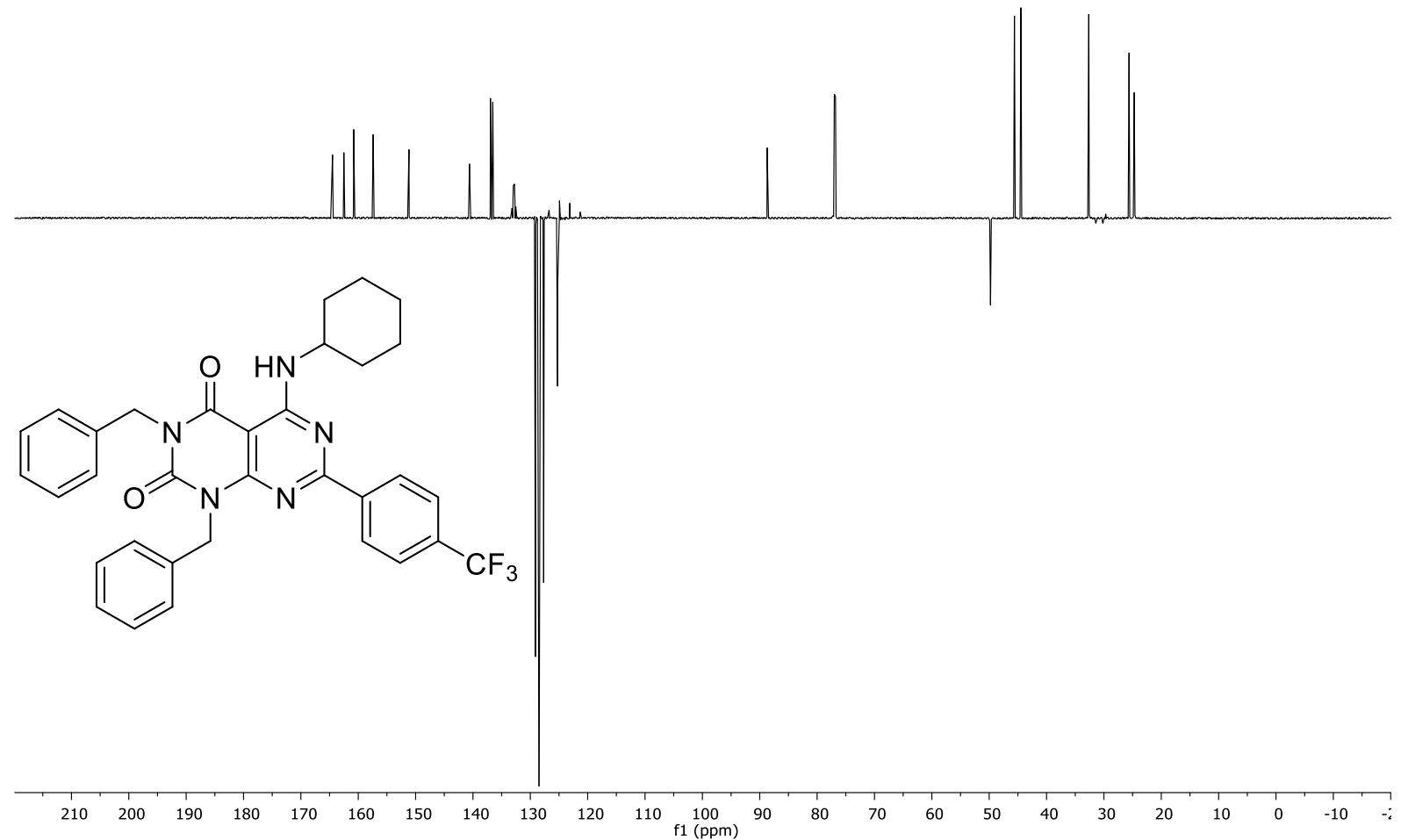




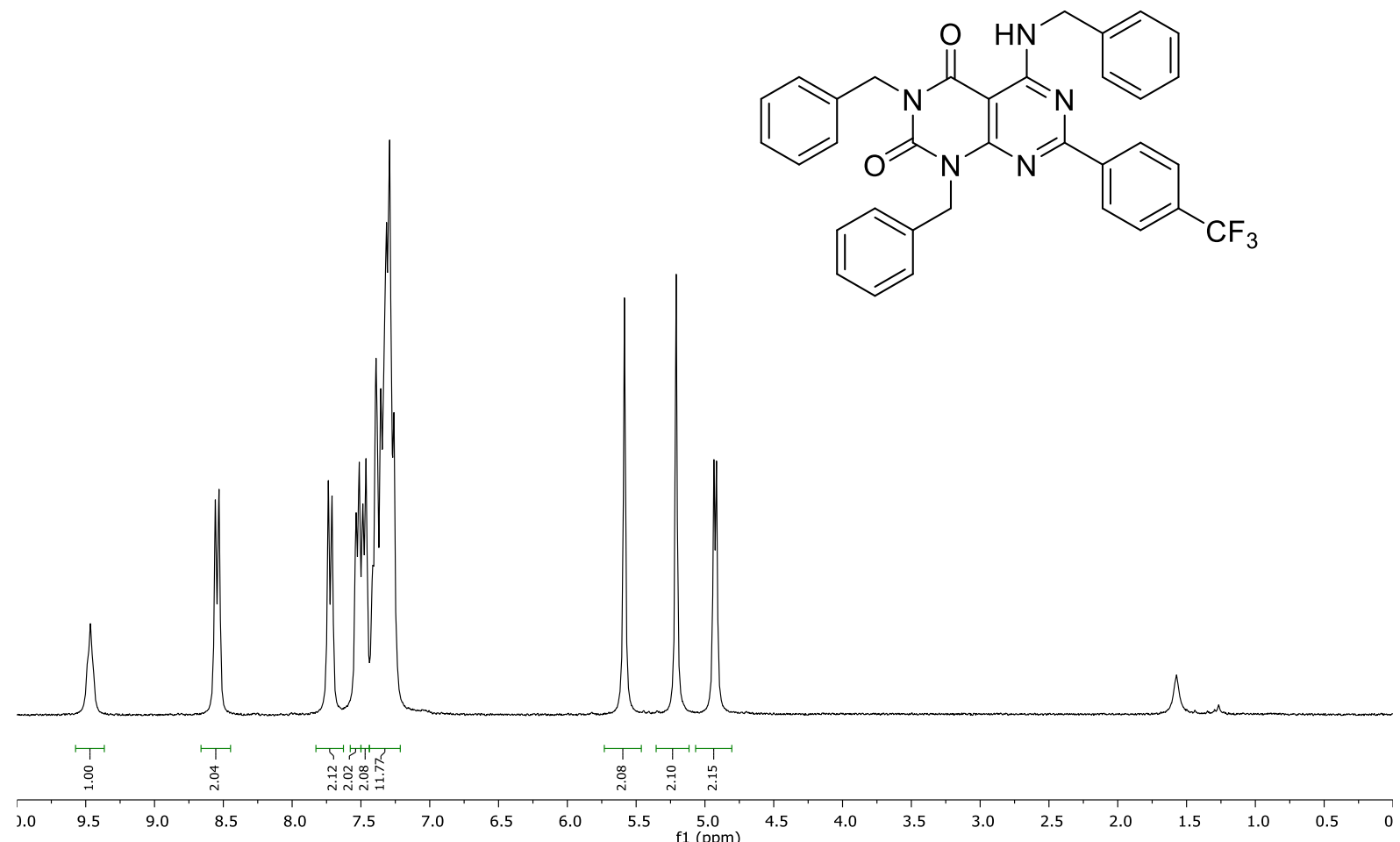

4f, ${ }^{1} \mathrm{H}$ NMR $\left(126 \mathrm{MHz}, \mathrm{CDCl}_{3}\right)$

HLo56.1.1id

|1।

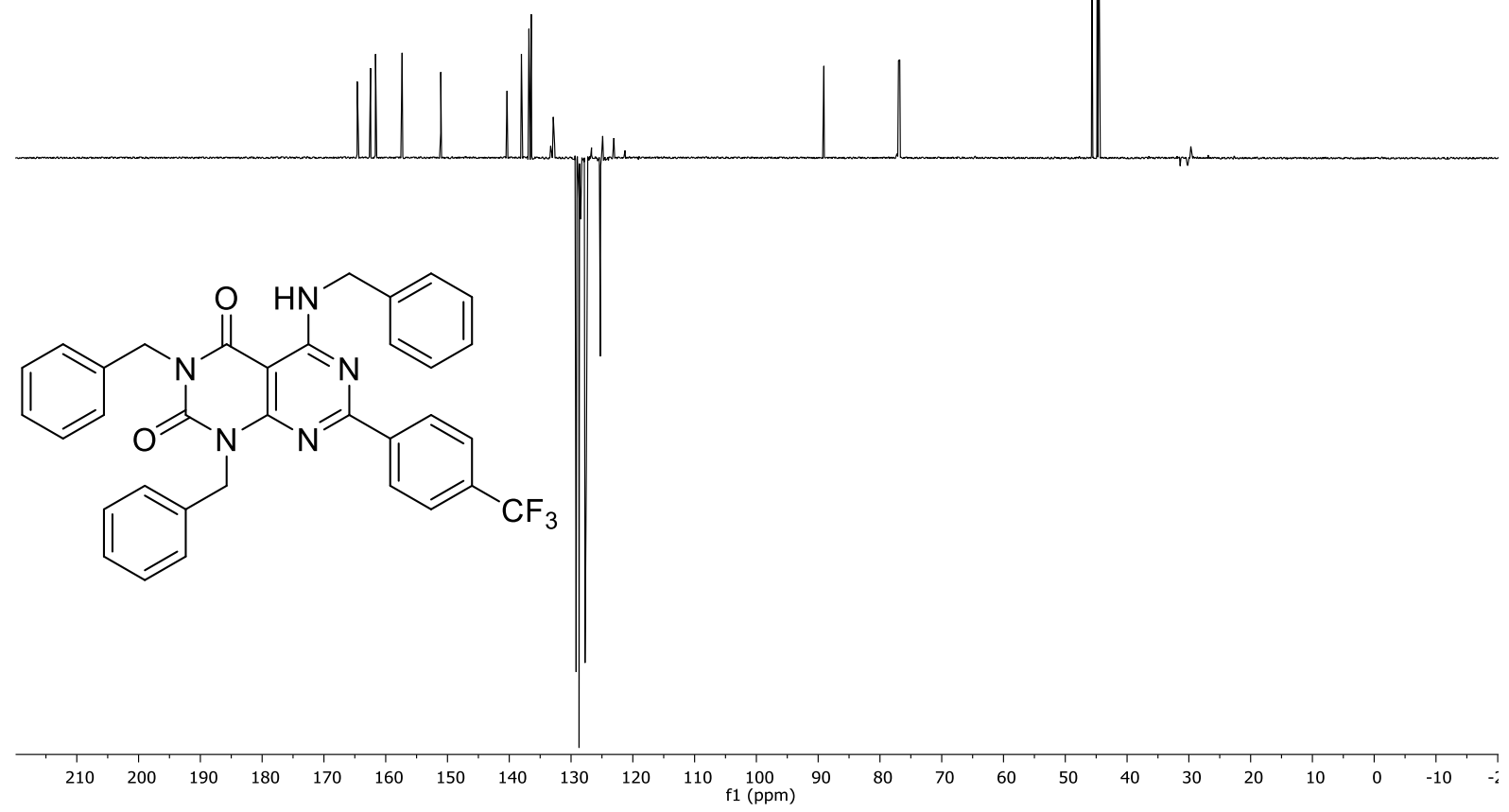


4g, ${ }^{1} \mathrm{H}$ NMR (300 MHz, $\mathrm{CDCl}_{3}$ )

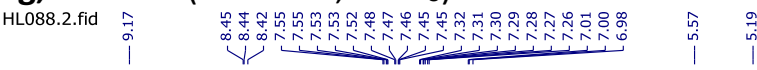<smiles>COc1ccc(-c2nc(NC(C)(C)C)c3c(=O)n(Cc4ccccc4)c(=O)n(Cc4ccccc4)c3n2)cc1</smiles>

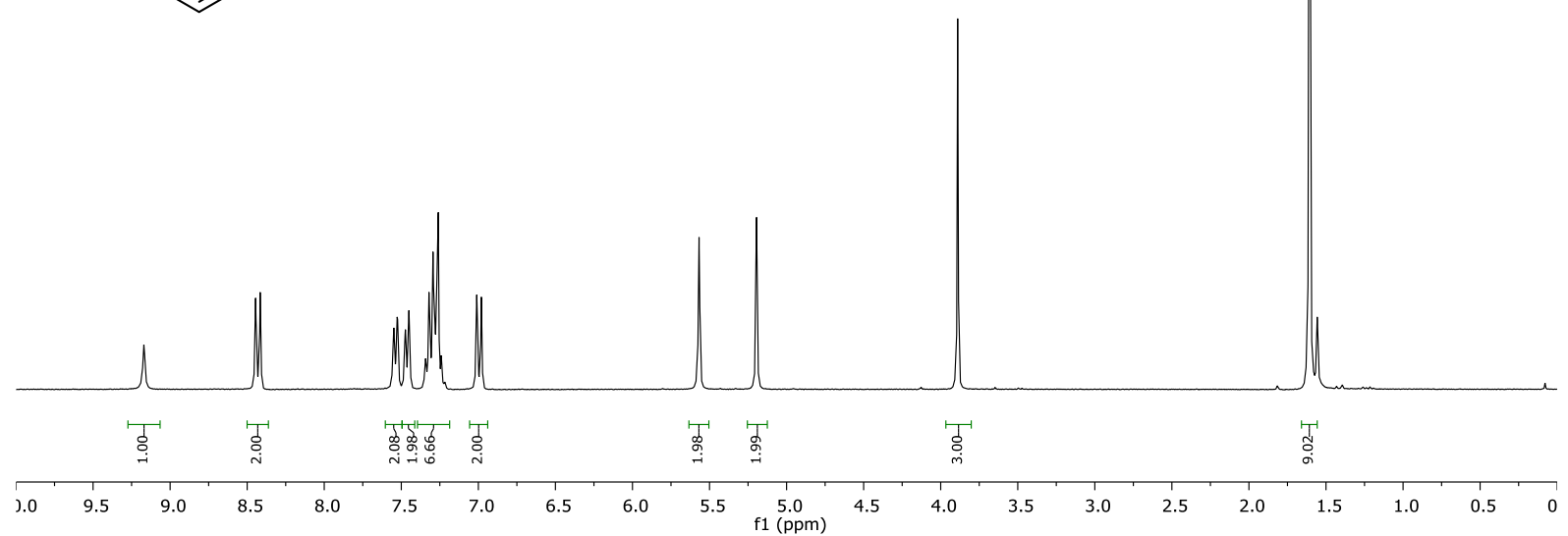

4g, ${ }^{1} \mathrm{H}$ NMR (126 MHz, $\mathrm{CDCl}_{3}$ )

HL088.1.fid<smiles>COc1ccc(-c2nc(NC(C)(C)C)c3c(=O)n(Cc4ccccc4)c(=O)n(Cc4ccccc4)c3n2)cc1</smiles>

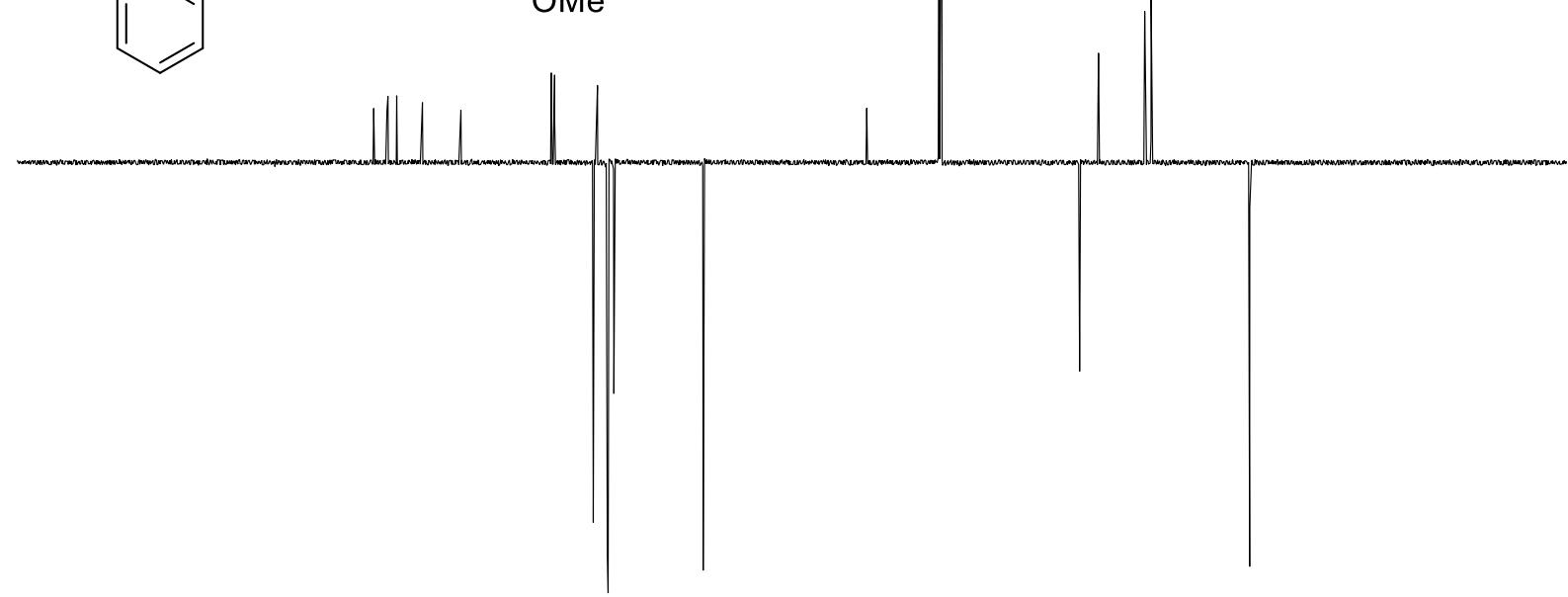

$\begin{array}{lllllllllllllllllllllllll}20 & 210 & 200 & 190 & 180 & 170 & 160 & 150 & 140 & 130 & 120 & 110 & 100 & 90 & 80 & 70 & 60 & 50 & 40 & 30 & 20 & 10 & 0 & -10 & -:\end{array}$ 
4h, ${ }^{1} \mathrm{H}$ NMR (300 MHz, $\mathrm{CDCl}_{3}$ )

HLo90.4.f倩

i.

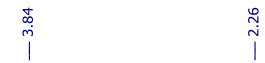<smiles>COc1ccc(-c2nc(Nc3c(C)cccc3C)c3c(=O)n(Cc4ccccc4)c(=O)n(Cc4ccccc4)c3n2)cc1</smiles>

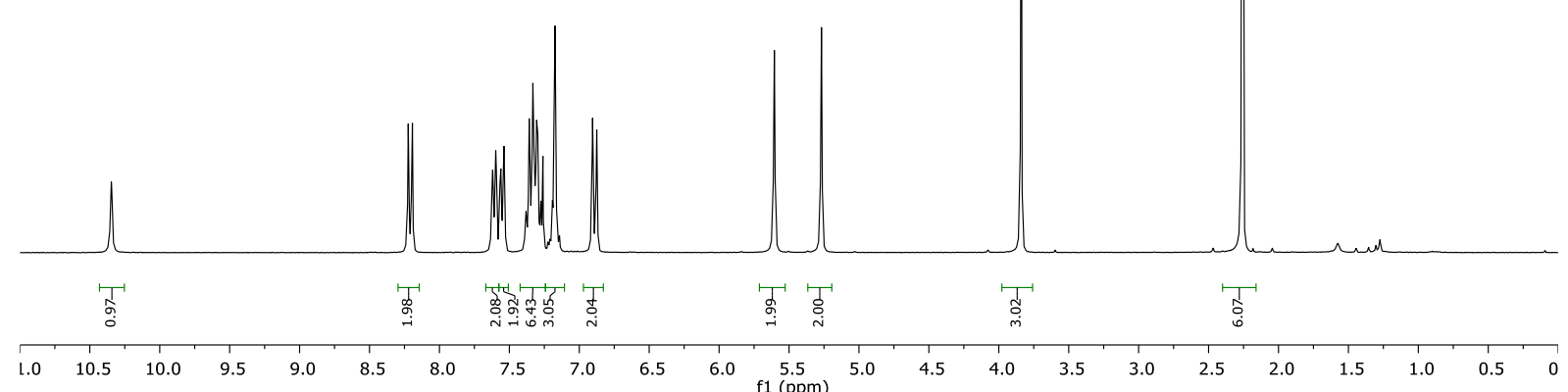

4h, ${ }^{1} \mathrm{H}$ NMR (126 MHz, $\mathrm{CDCl}_{3}$ )

HL090.1.fid

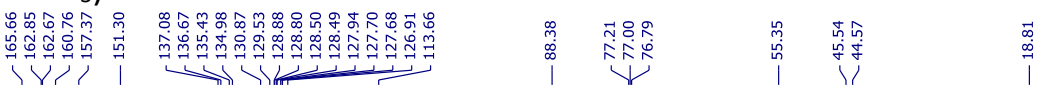

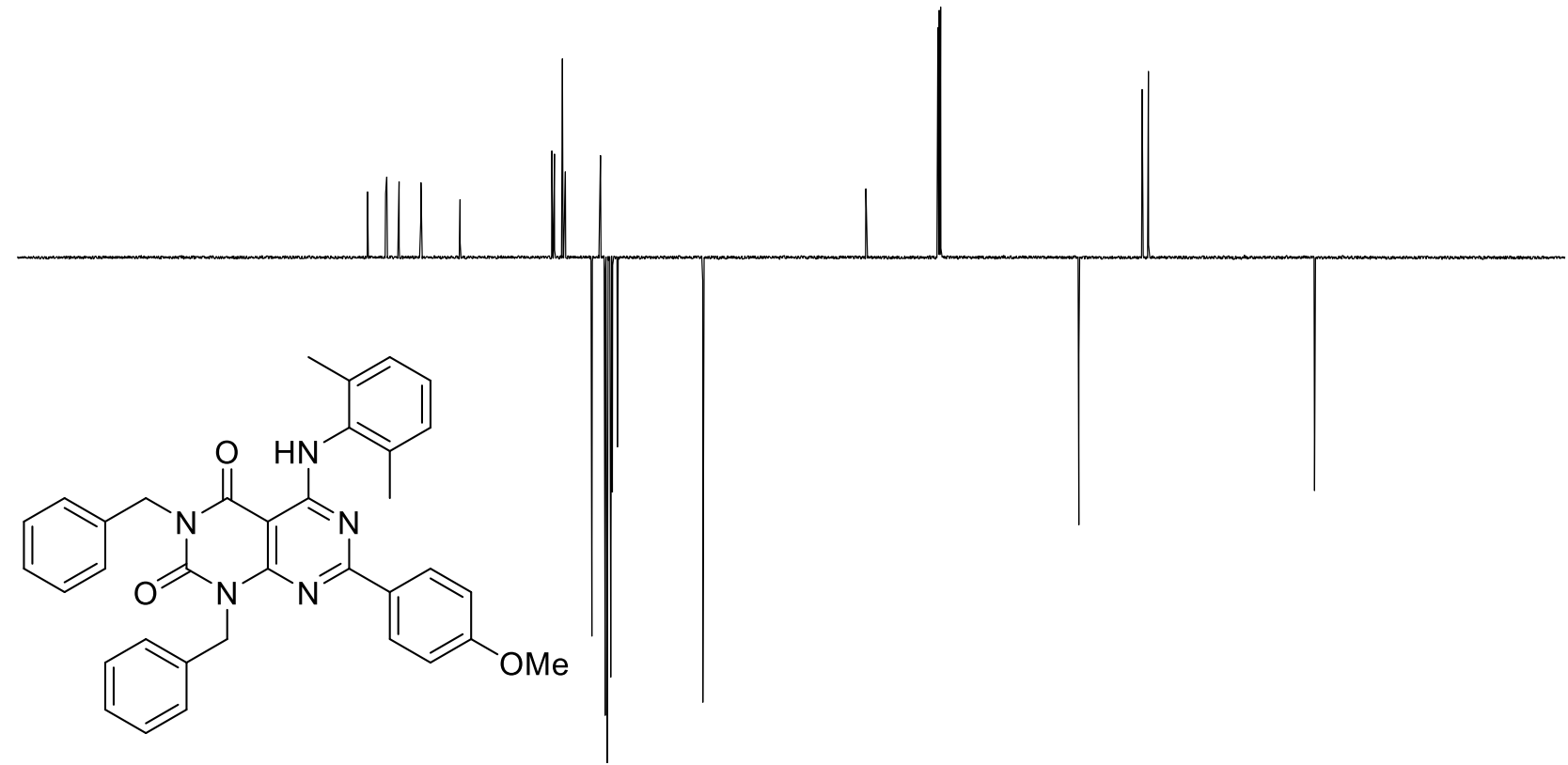

$\begin{array}{lllllllllllllllllllllllllllll}210 & 200 & 190 & 180 & 170 & 160 & 150 & 140 & 130 & 120 & 110 & 100 & 90 & 80 & 70 & 60 & 50 & 40 & 30 & 20 & 10 & 0 & -10 & -\end{array}$ 
4i, ${ }^{1} \mathrm{H}$ NMR $\left(300 \mathrm{MHz}, \mathrm{CDCl}_{3}\right)$

HLo89.3.fid

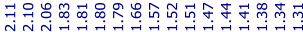

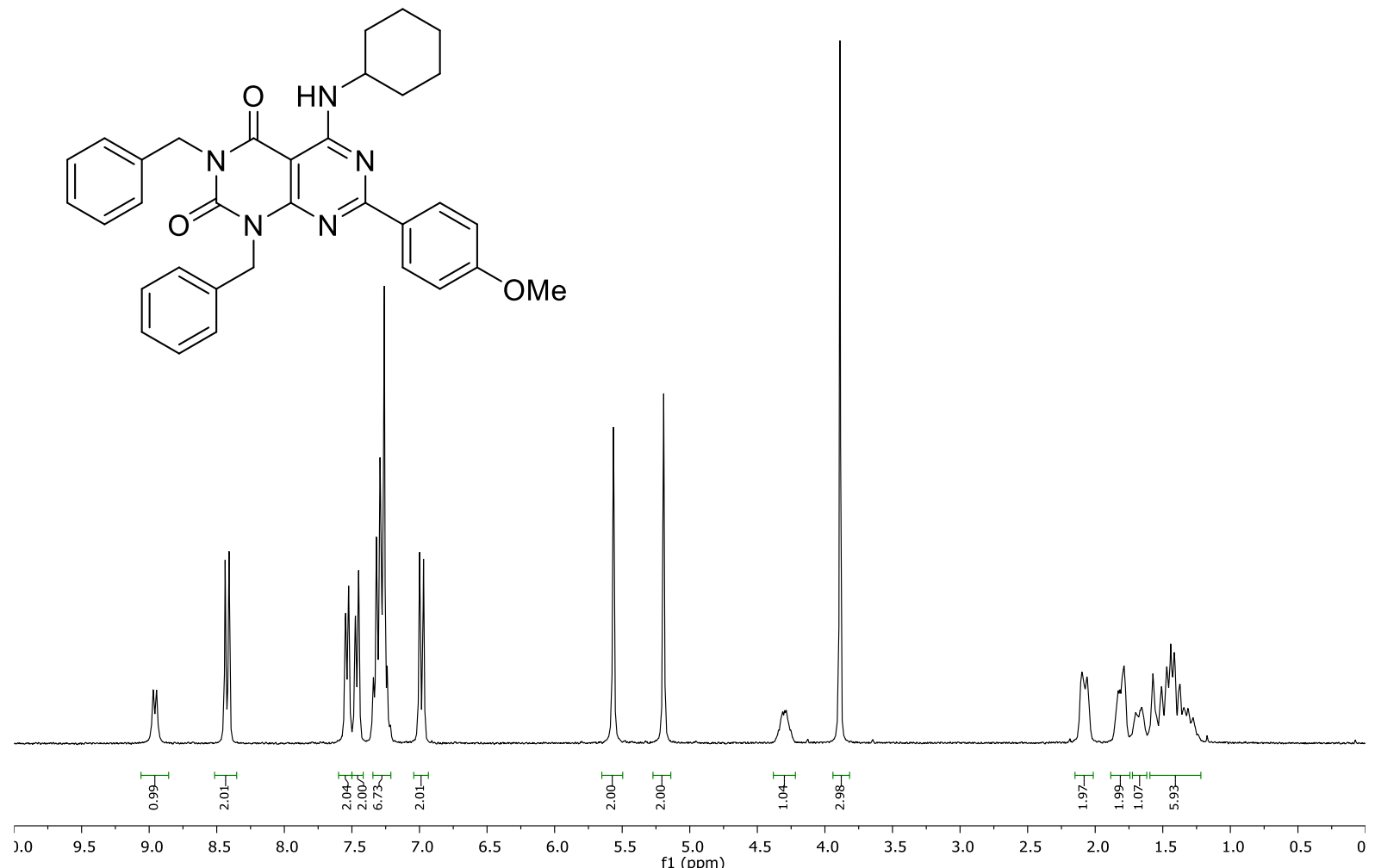

4i, ${ }^{1} \mathrm{H}$ NMR (126 MHz, $\mathrm{CDCl}_{3}$ )

HL089.3.fid

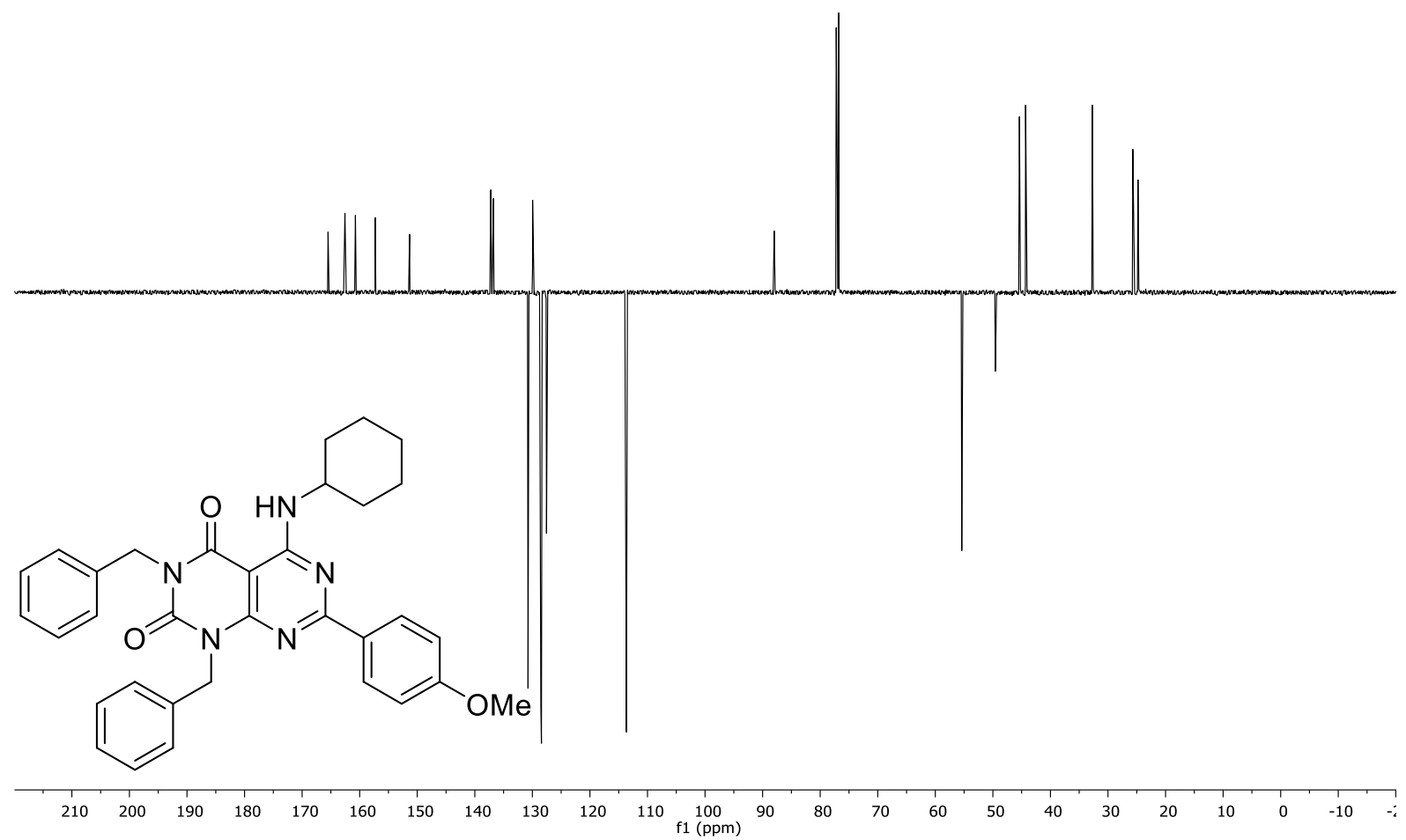


5a, ${ }^{1} \mathrm{H} \mathrm{NMR}\left(300 \mathrm{MHz}, \mathrm{CDCl}_{3}\right)$

HL082.1.fid 号<smiles>Cc1nc(NC(C)(C)C)c2c(=O)n(Cc3ccccc3)c(=O)n(Cc3ccccc3)c2n1</smiles>

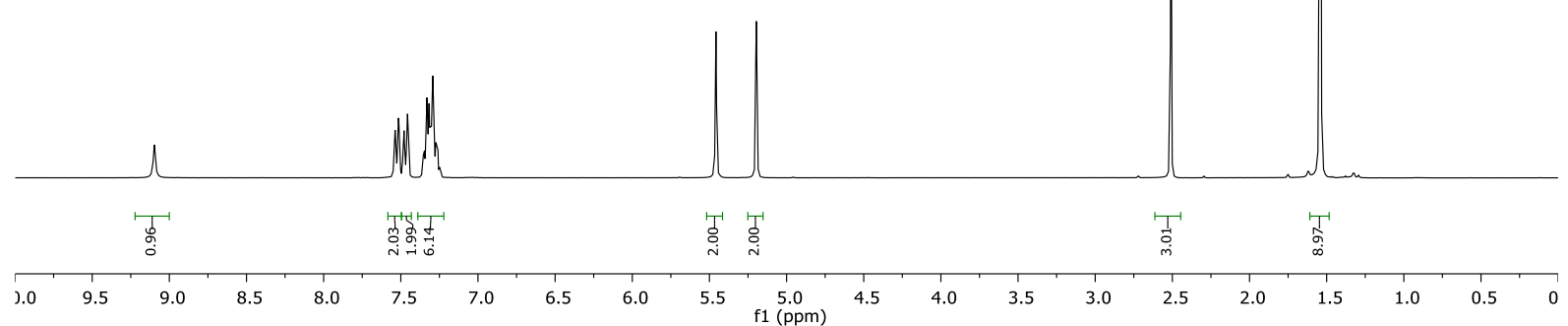

5a, ${ }^{1} \mathrm{H}$ NMR (126 MHz, $\mathrm{CDCl}_{3}$ )

HLo82.1.fid

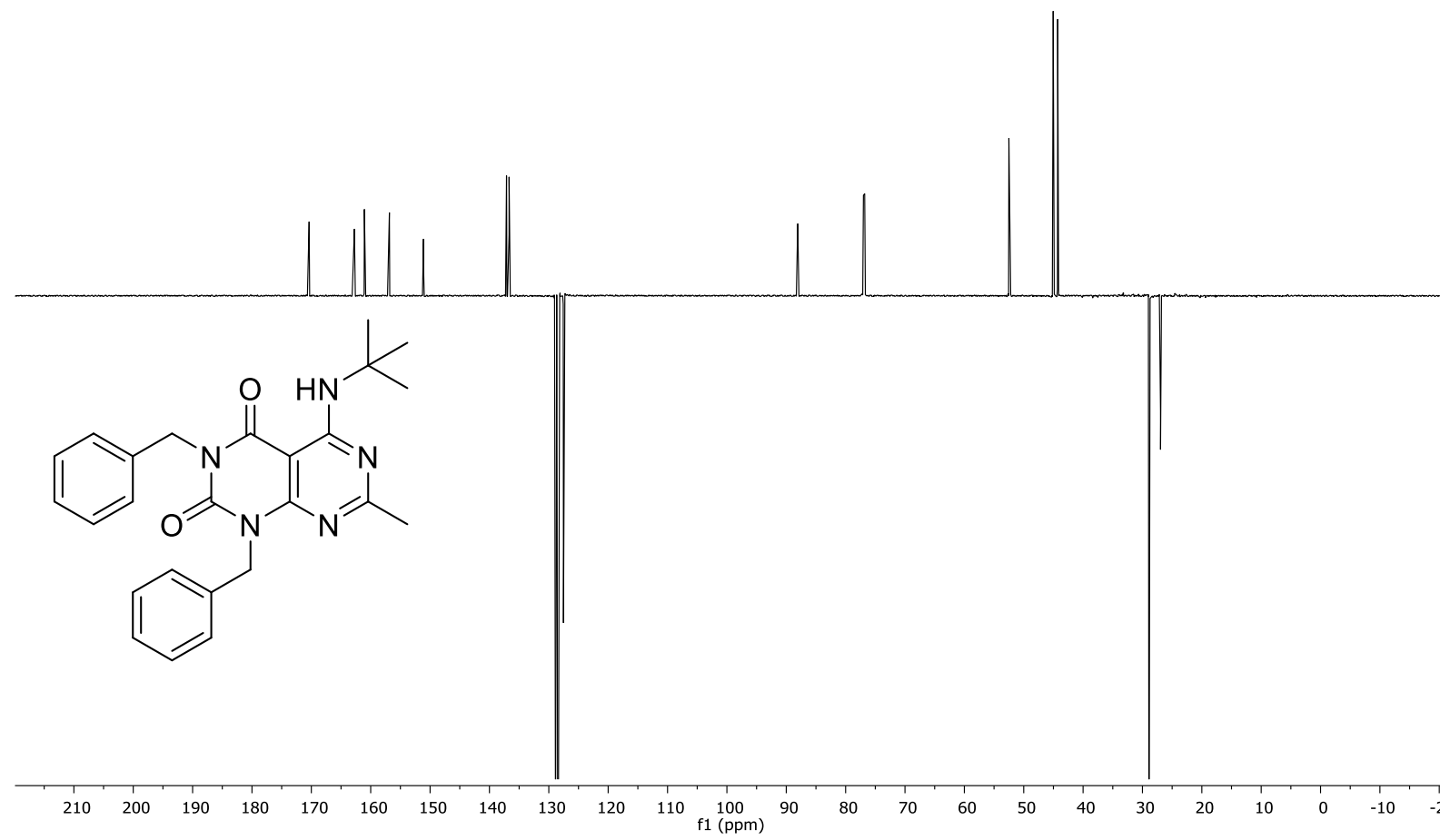


5b, ${ }^{1} \mathrm{H}$ NMR (300 MHz, $\mathrm{CDCl}_{3}$ )

HL131.2.fid

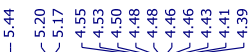

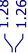<smiles>Cc1nc(NC(C)C)c2c(=O)n(Cc3ccccc3)c(=O)n(Cc3ccccc3)c2n1</smiles>

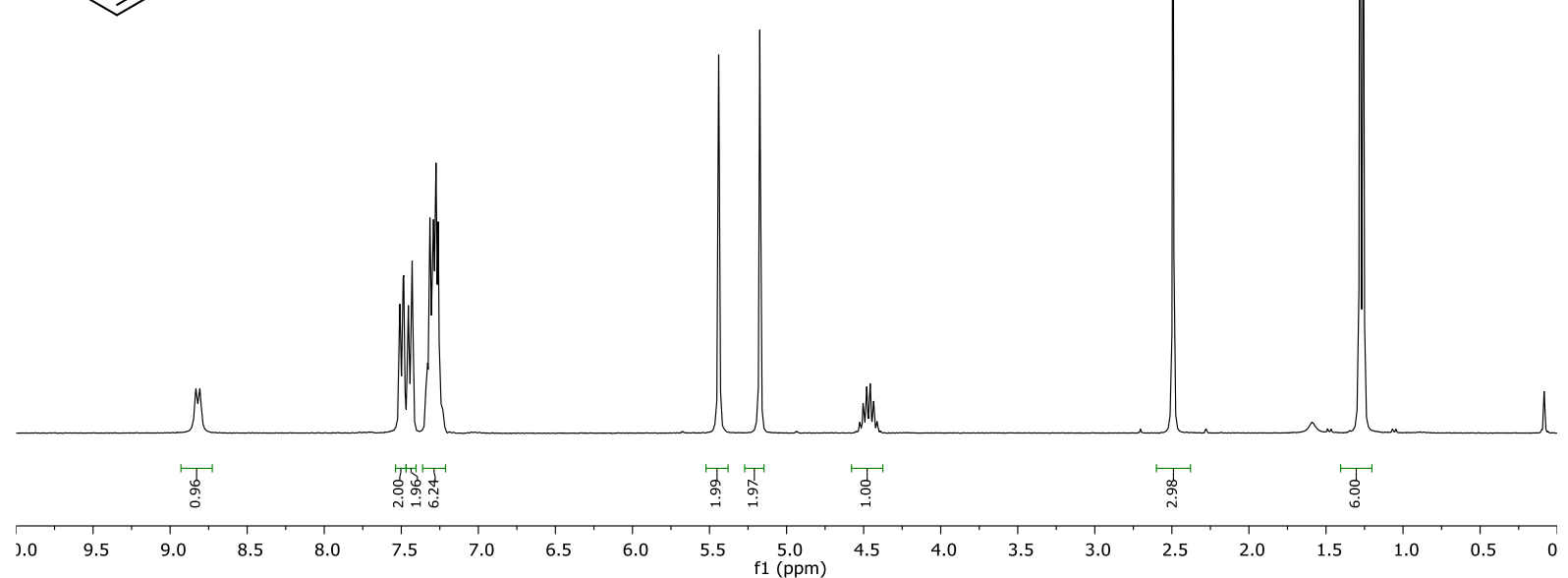

5b, ${ }^{1} \mathrm{H}$ NMR (126 MHz, $\mathrm{CDCl}_{3}$ )

HL131.1.fid

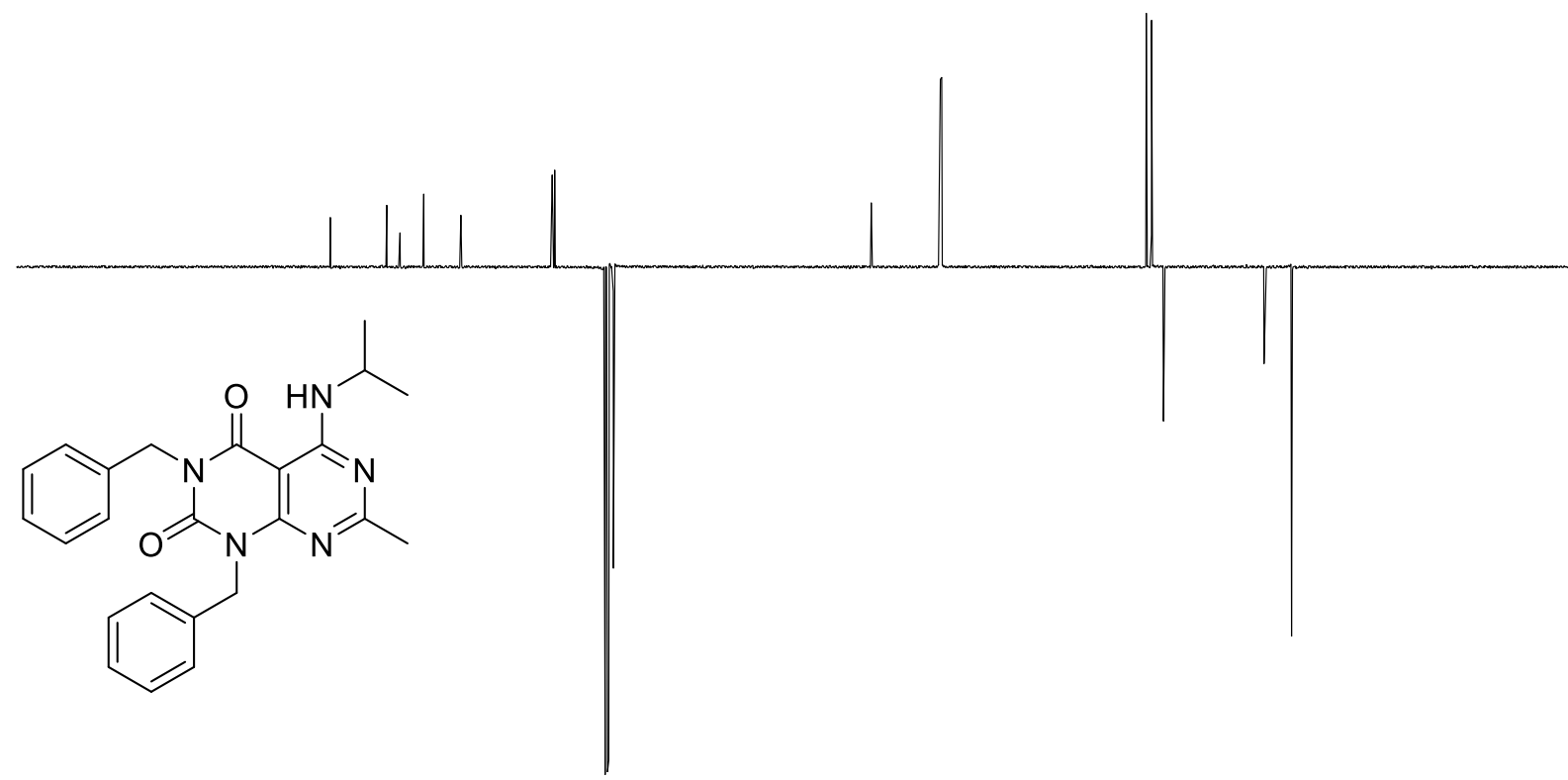

$\begin{array}{llllllllllllllllllllllll}210 & 200 & 190 & 180 & 170 & 160 & 150 & 140 & 130 & 120 & 110 & \begin{array}{c}100 \\ \mathrm{f} 1(\mathrm{ppm})\end{array} & 90 & 80 & 70 & 60 & 50 & 40 & 30 & 20 & 10 & 0 & -10 & -\mathrm{i}\end{array}$ 
<smiles>Cc1nc(NC2CCCCC2)c2c(=O)n(Cc3ccccc3)c(=O)n(Cc3ccccc3)c2n1</smiles>

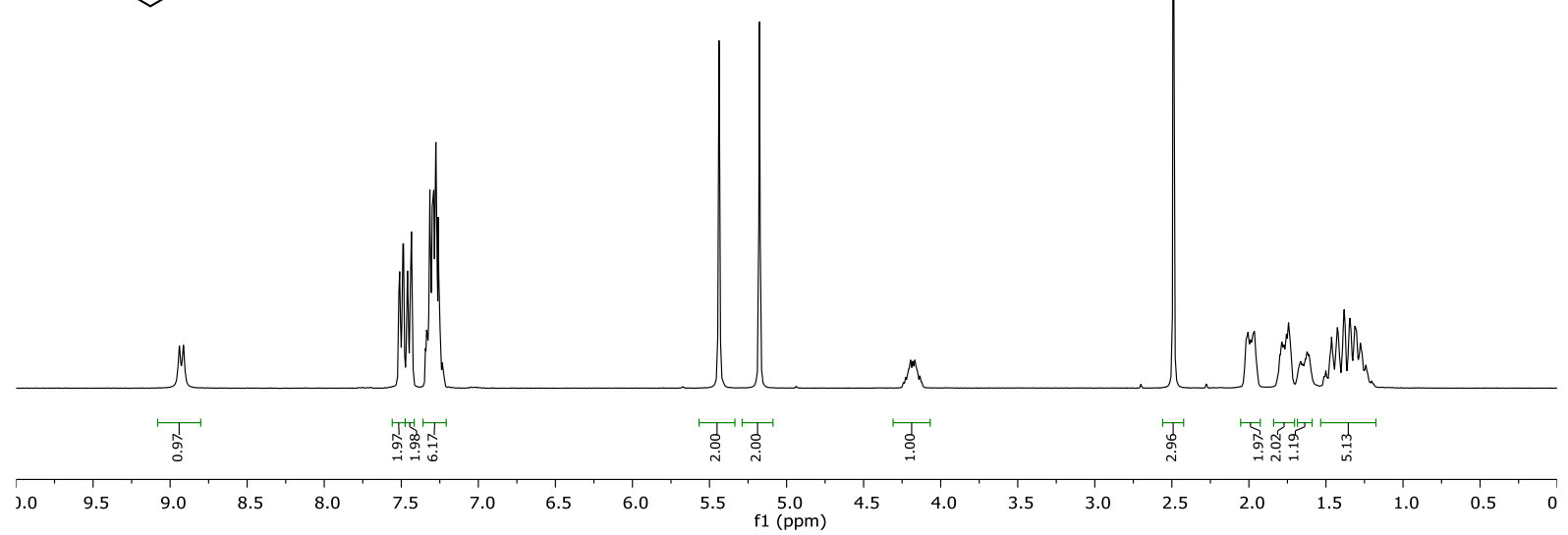

5c, ${ }^{1} \mathrm{H}$ NMR $\left(126 \mathrm{MHz}, \mathrm{CDCl}_{3}\right)$

HL113.1.fid
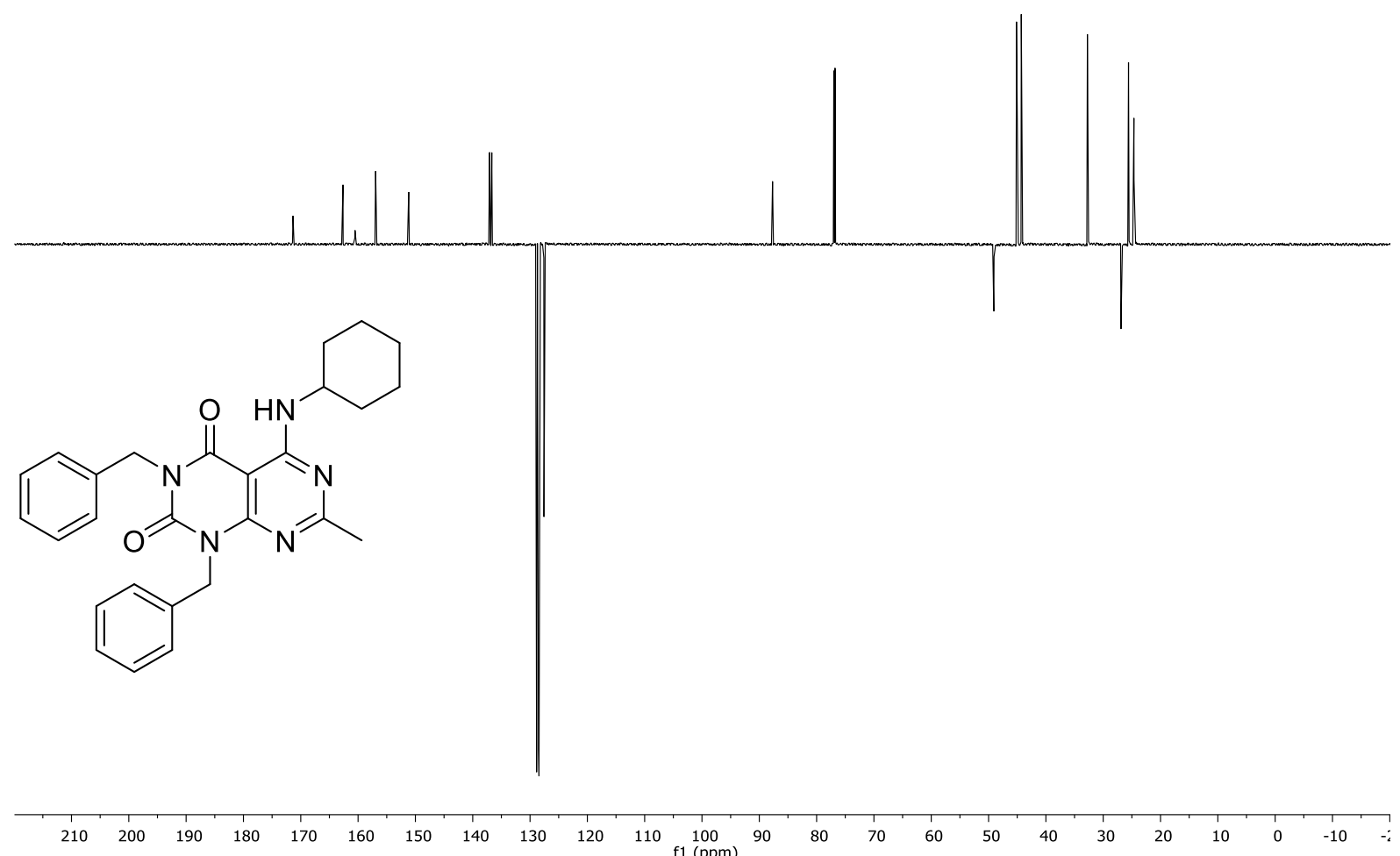
5d, ${ }^{1} \mathrm{H}$ NMR (300 MHz, $\left.\mathrm{CDCl}_{3}\right)$

HL129.1.fid

在

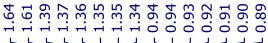<smiles>CCCCCNc1nc(C)nc2c1c(=O)n(Cc1ccccc1)c(=O)n2Cc1ccccc1</smiles>

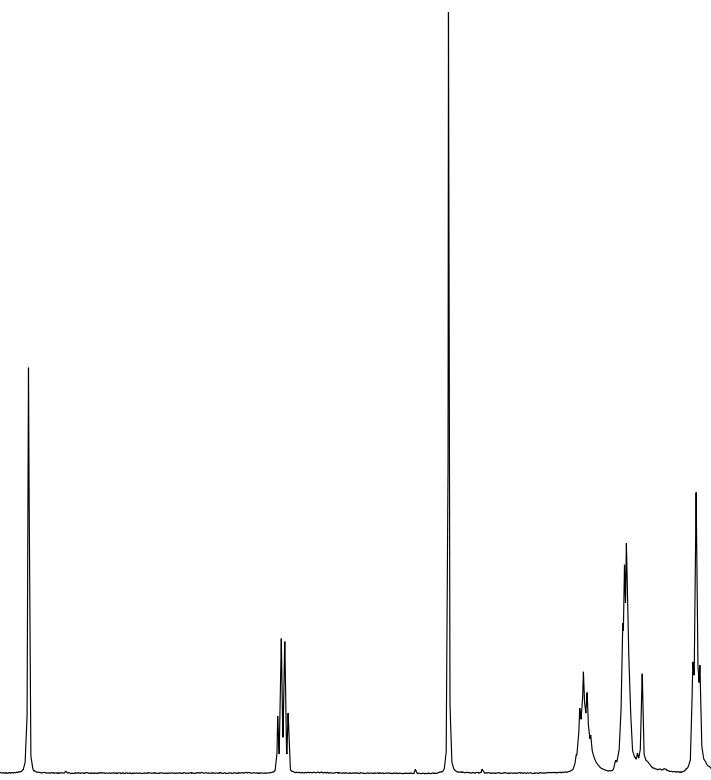

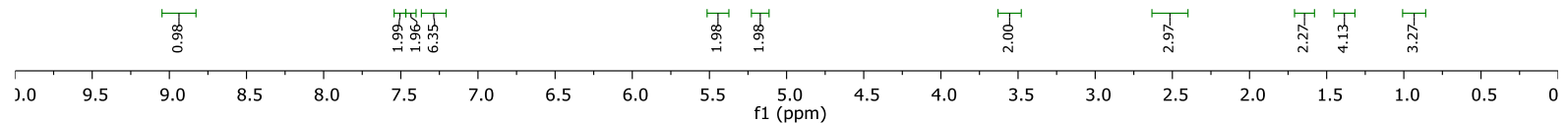

5d, ${ }^{1} \mathrm{H}$ NMR (126 MHz, $\mathrm{CDCl}_{3}$ )

HL129.1.fid<smiles>CCCCCNc1nc(C)nc2c1c(=O)n(Cc1ccccc1)c(=O)n2Cc1ccccc1</smiles>

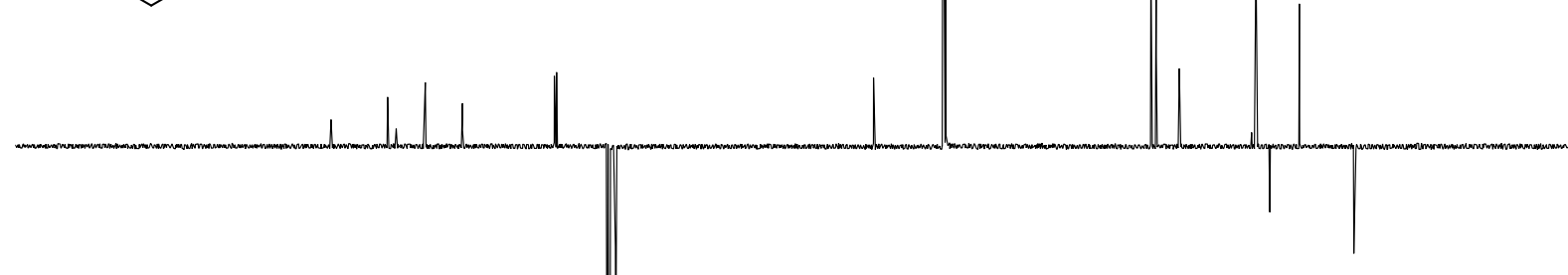

$\begin{array}{llllllllllllllllllllllll}210 & 200 & 190 & 180 & 170 & 160 & 150 & 140 & 130 & 120 & 110 & \begin{array}{c}100 \\ \mathrm{f} 1(\mathrm{ppm})\end{array} & 90 & 80 & 70 & 60 & 50 & 40 & 30 & 20 & 10 & 0 & -10 & -:\end{array}$ 
5e, ${ }^{1} \mathrm{H} \mathrm{NMR}\left(300 \mathrm{MHz}, \mathrm{CDCl}_{3}\right)$

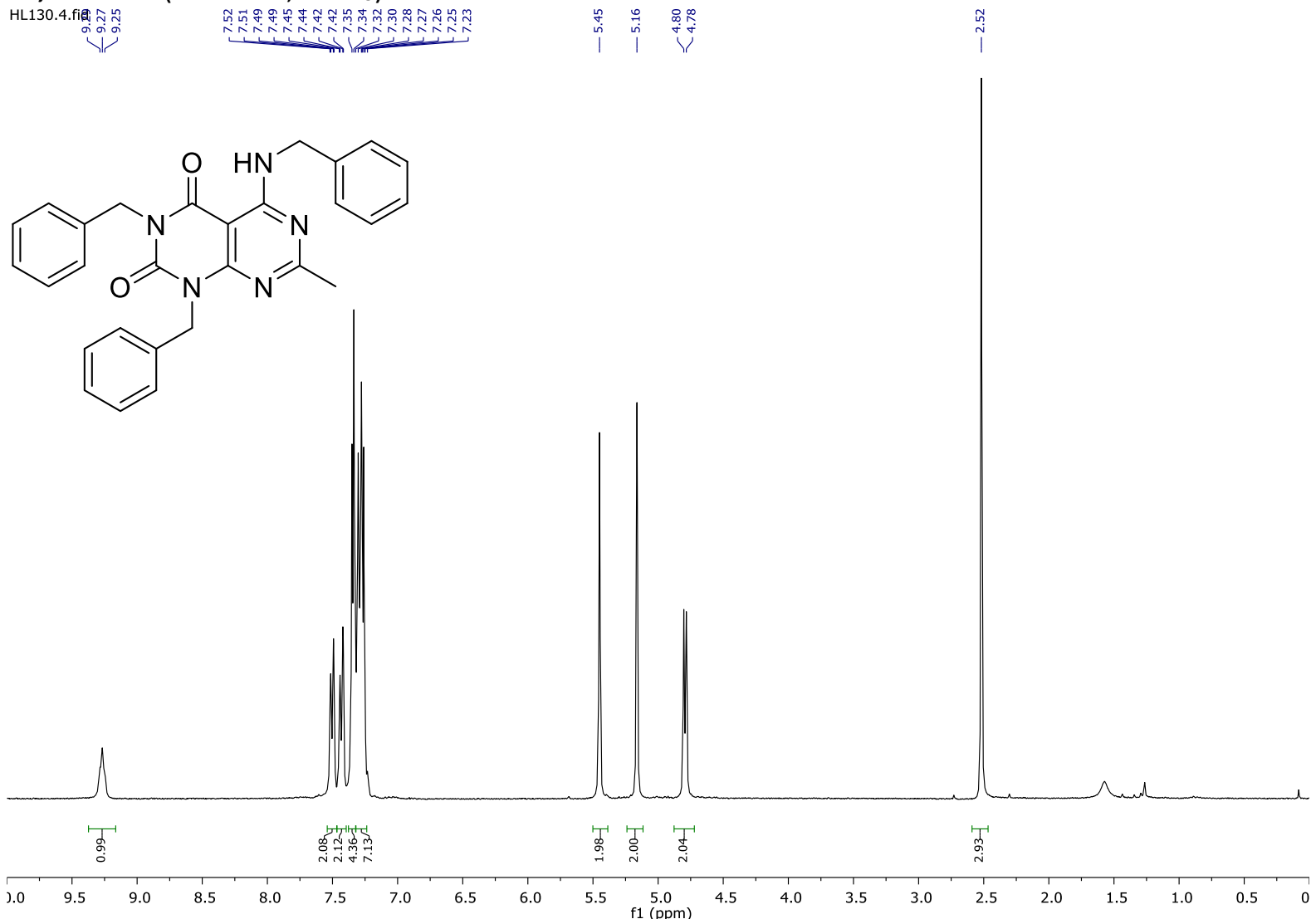

5e, ${ }^{1} \mathrm{H}$ NMR (126 MHz, $\mathrm{CDCl}_{3}$ )

HL130.1.fid<smiles>Cc1nc(NCc2ccccc2)c2c(=O)n(Cc3ccccc3)c(=O)n(Cc3ccccc3)c2n1</smiles>

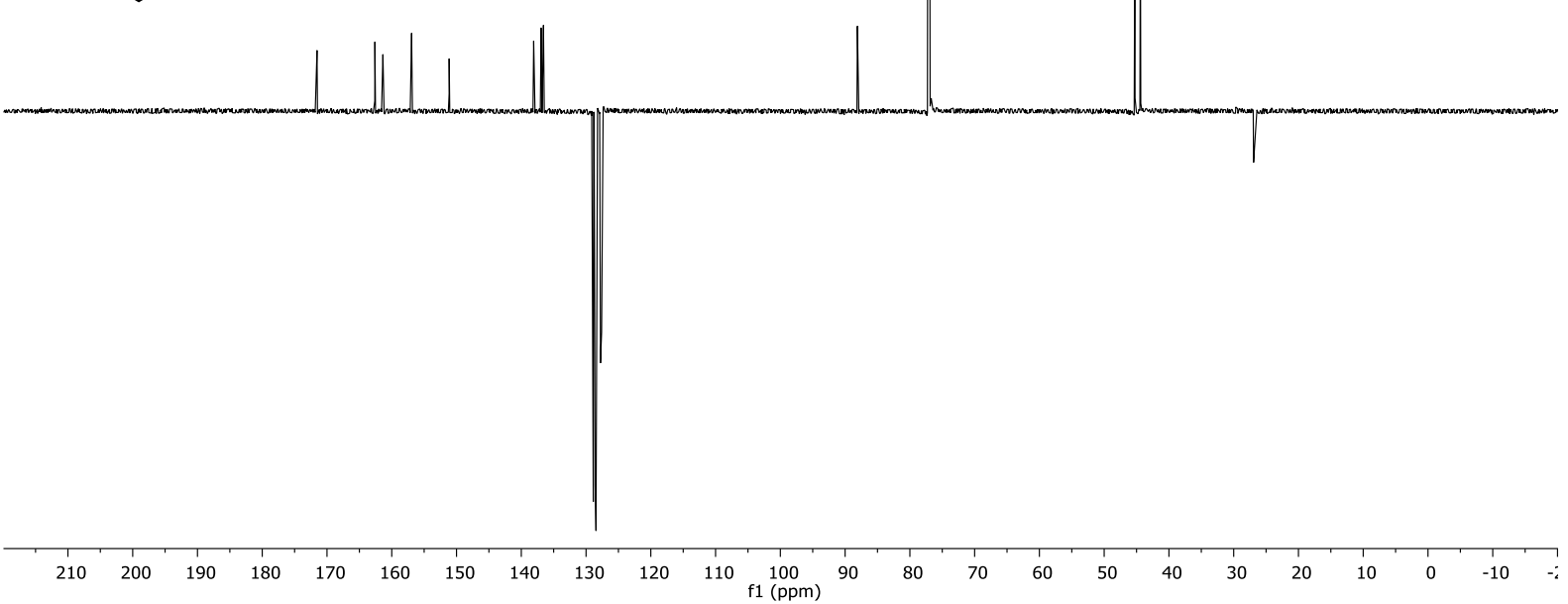


5f, ${ }^{1} \mathrm{H}$ NMR (300 MHz, $\left.\mathrm{CDCl}_{3}\right)$

HL132.2.fid<smiles>Cc1nc(NC(C)(C)CC(C)(C)C)c2c(=O)n(Cc3ccccc3)c(=O)n(Cc3ccccc3)c2n1</smiles>

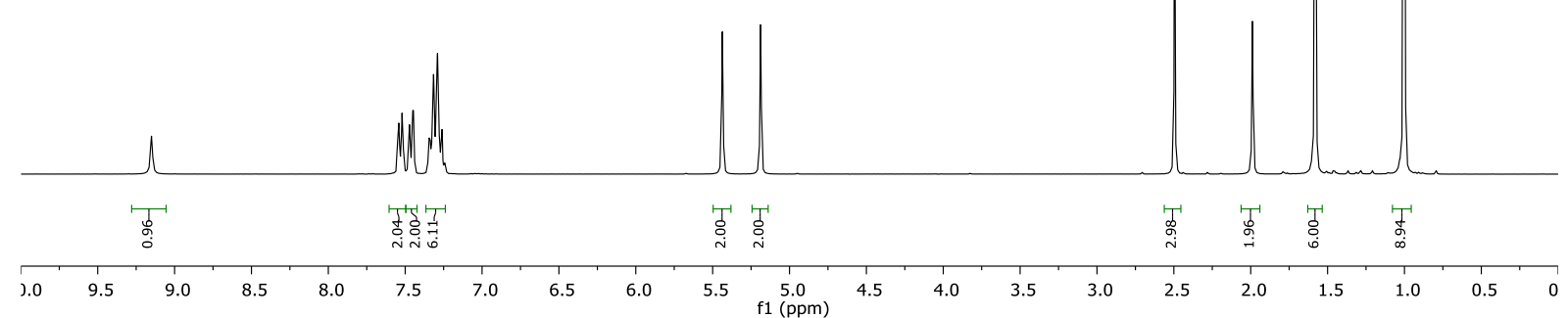

5f, ${ }^{1} \mathrm{H}$ NMR (126 MHz, $\mathrm{CDCl}_{3}$ )

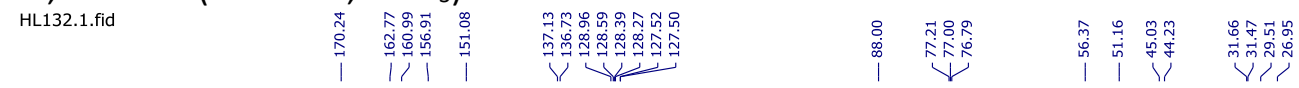

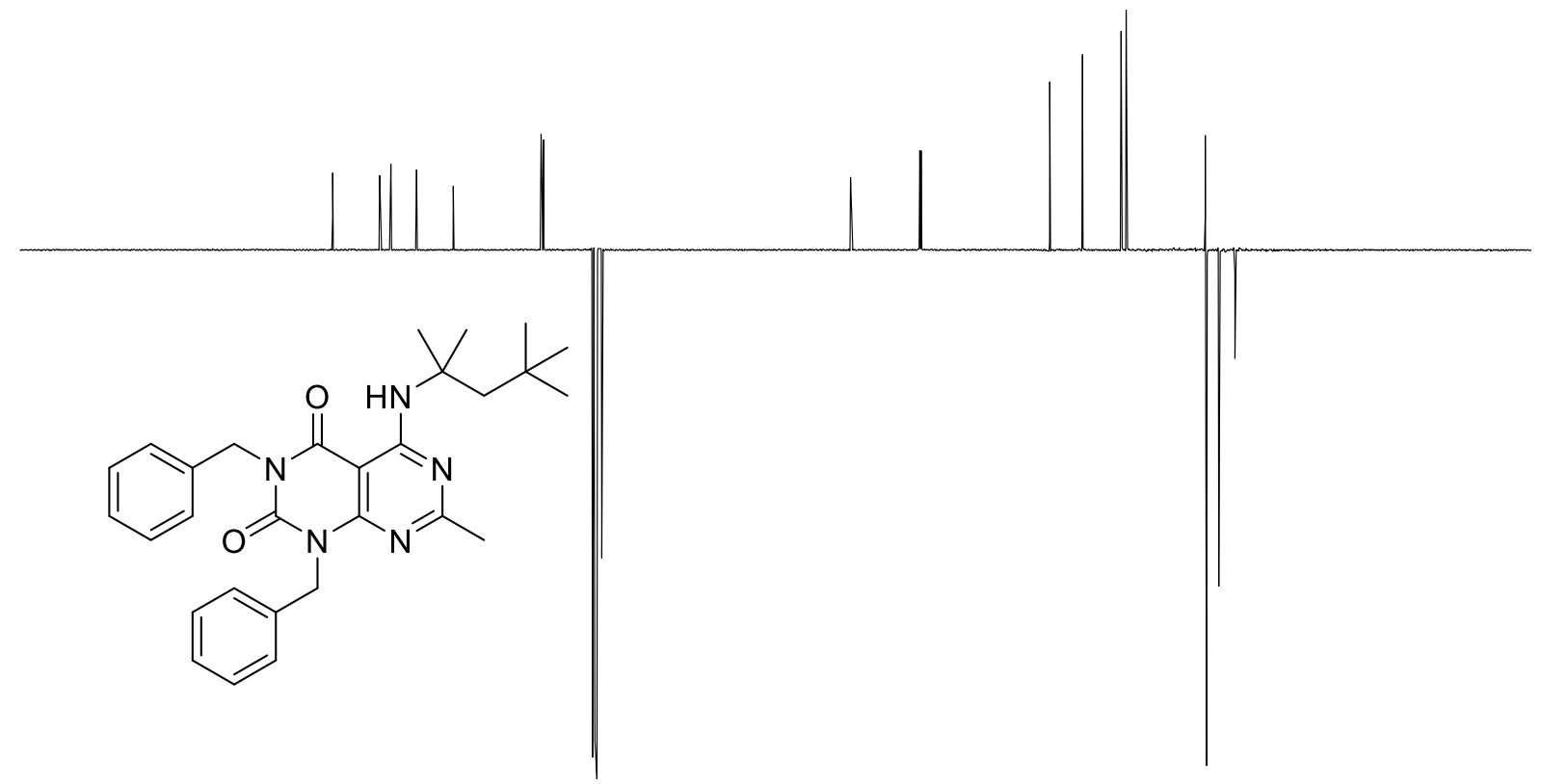

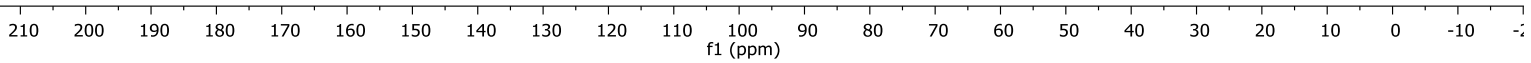


5g, ${ }^{1} \mathrm{H}$ NMR (300 MHz, $\mathrm{CDCl}_{3}$ )

HL135.4.fid

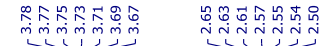<smiles>Cc1nc(NCCN2CCOCC2)c2c(=O)n(Cc3ccccc3)c(=O)n(Cc3ccccc3)c2n1</smiles>

宊

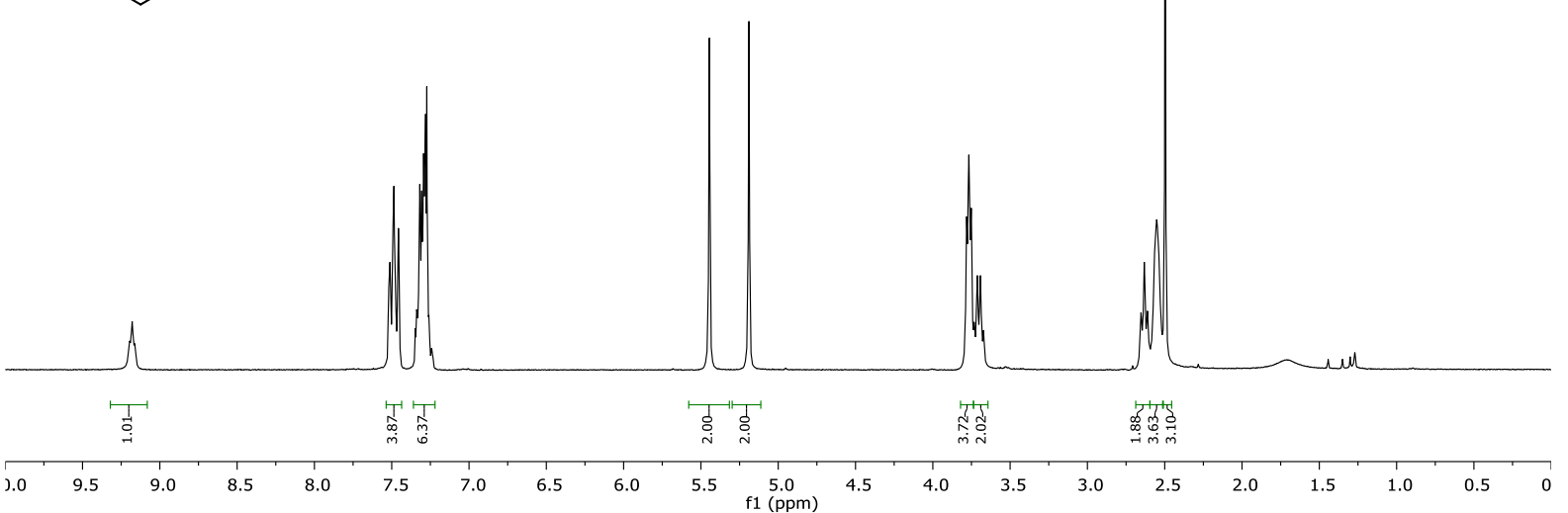

5g, ${ }^{1} \mathrm{H}$ NMR $\left(126 \mathrm{MHz}, \mathrm{CDCl}_{3}\right)$ HL135.1.fid

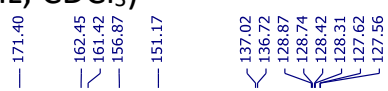

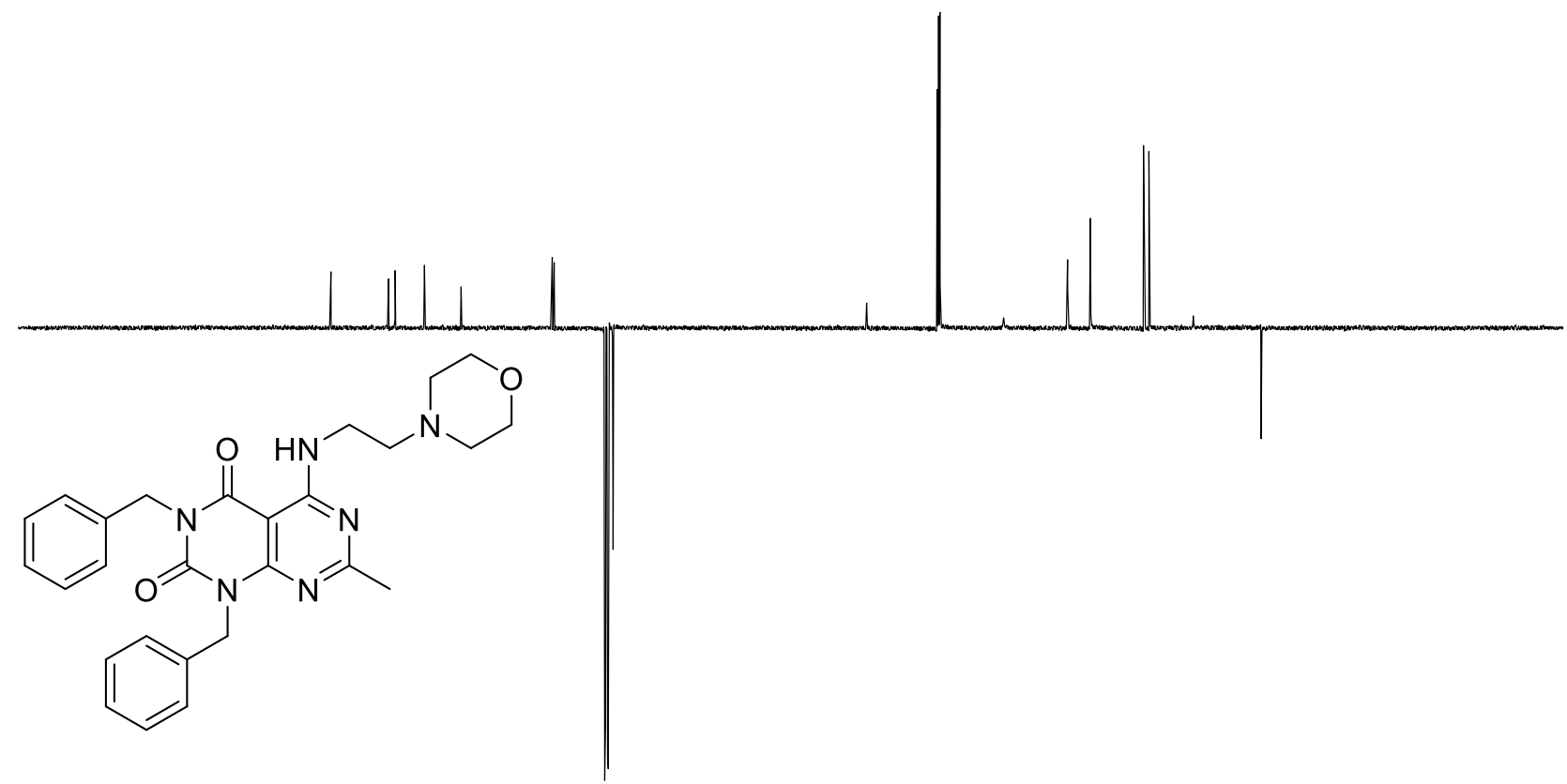

$\begin{array}{lllllllllllllllllllllllll}210 & 200 & 190 & 180 & 170 & 160 & 150 & 140 & 130 & 120 & 110 & 100 & 90 & 80 & 70 & 60 & 50 & 40 & 30 & 20 & 10 & 0 & -10 & -\end{array}$ 
5h, ${ }^{1} \mathrm{H}$ NMR (300 MHz, $\mathrm{CDCl}_{3}$ )

HL114.5.fifich<smiles>Cc1nc(Nc2c(C)cccc2C)c2c(=O)n(Cc3ccccc3)c(=O)n(Cc3ccccc3)c2n1</smiles>

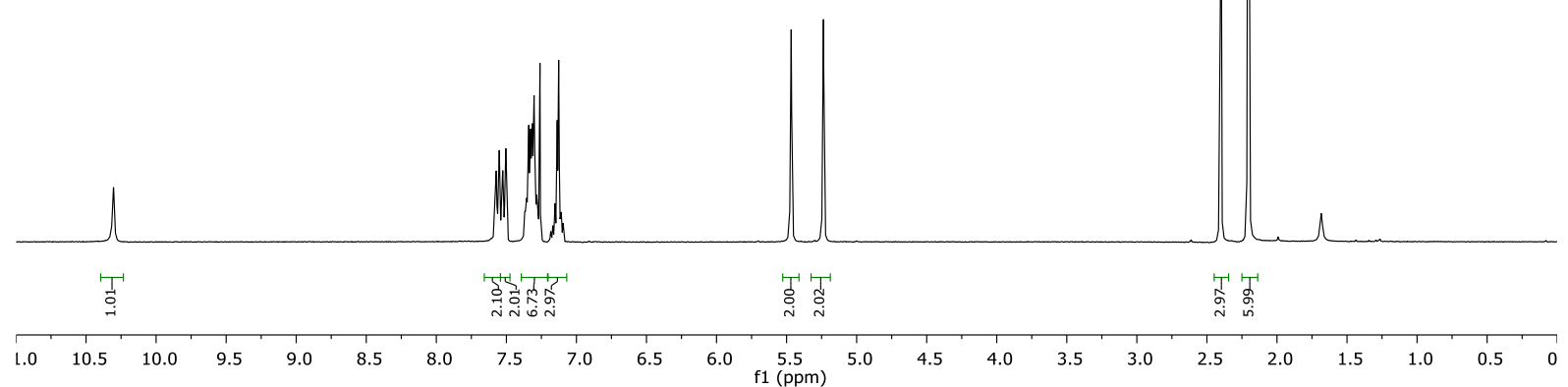

5h, ${ }^{1} \mathrm{H}$ NMR (126 MHz, $\mathrm{CDCl}_{3}$ )

HL14.1.1.

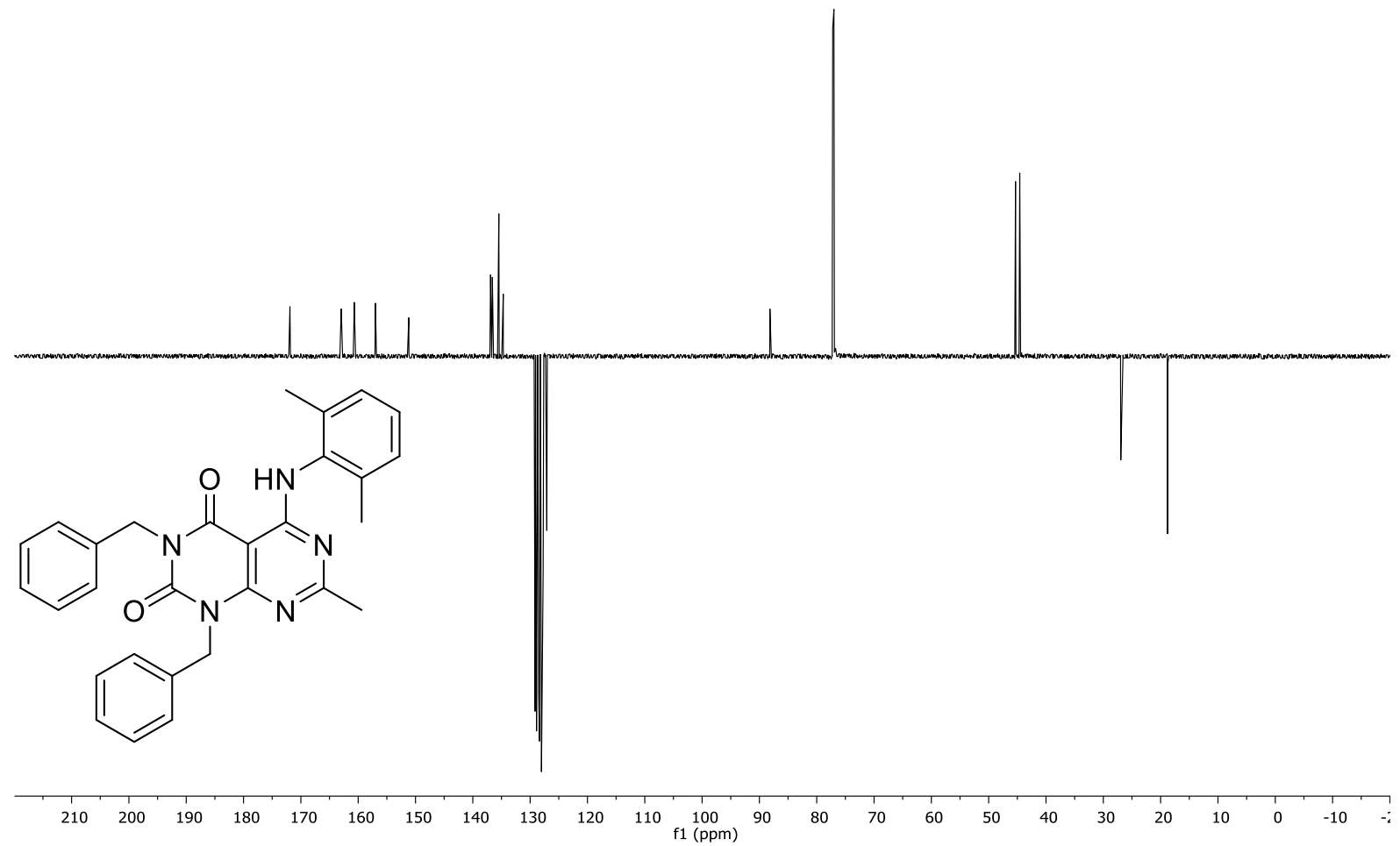


5j, ${ }^{1} \mathrm{H}$ NMR $\left(300 \mathrm{MHz}, \mathrm{CDCl}_{3}\right)$

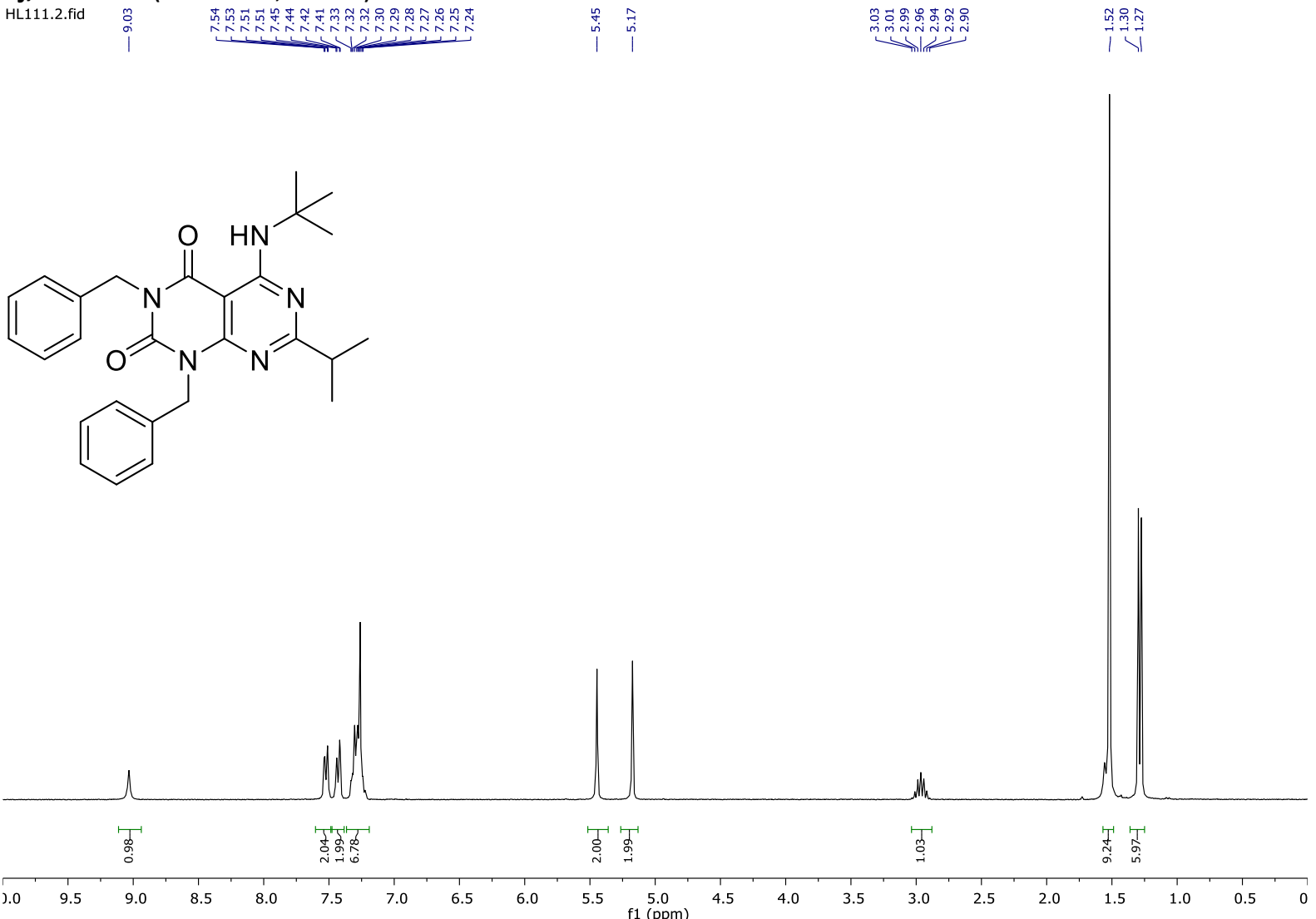

5j, ${ }^{1} \mathrm{H}$ NMR (126 MHz, $\left.\mathrm{CDCl}_{3}\right)$

HL111.1.fid

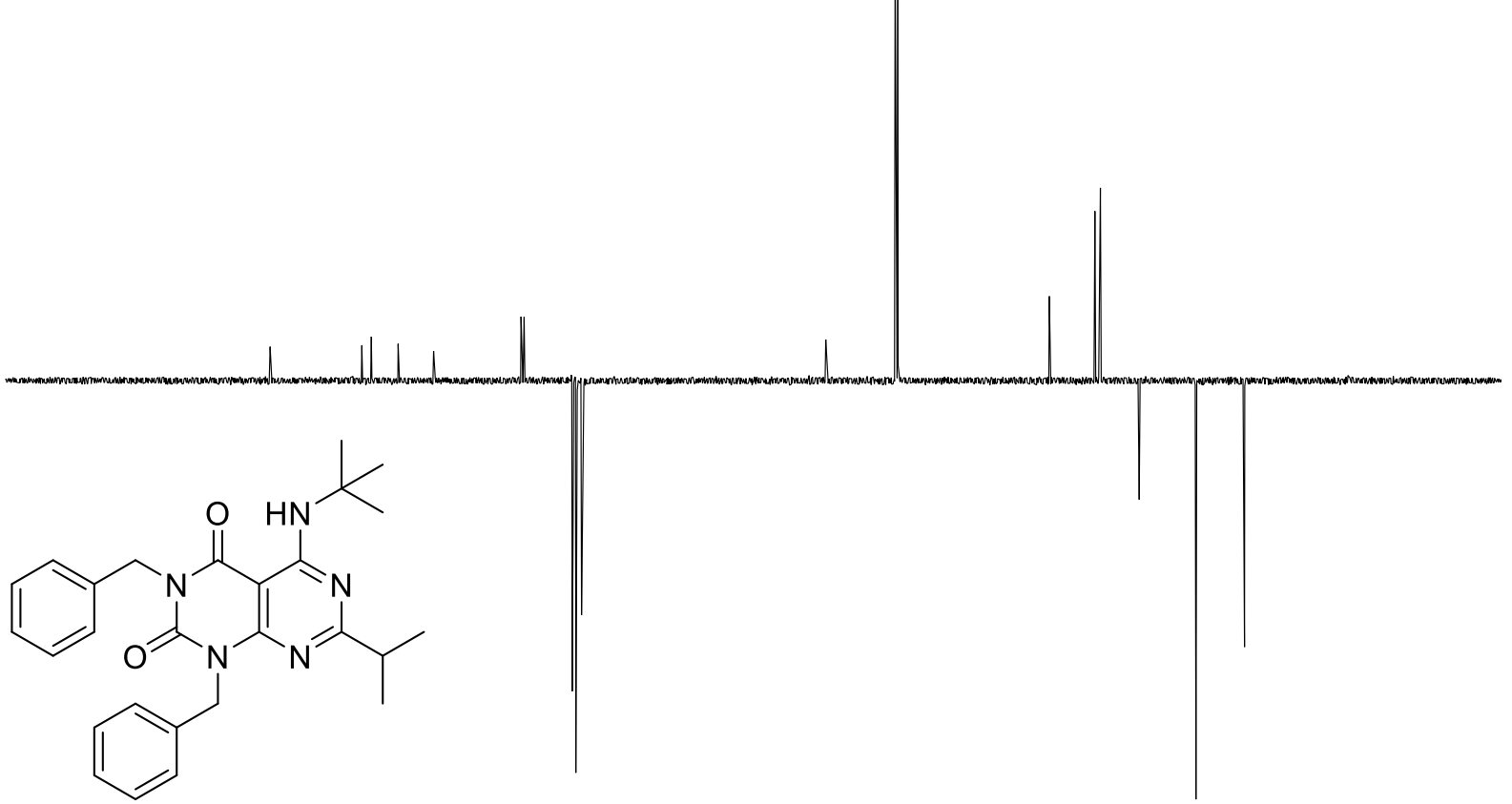

$\begin{array}{lllllllllllllllllllllllllll}20 & 210 & 200 & 190 & 180 & 170 & 160 & 150 & 140 & 130 & 120 & 110 & 100 & 90 & 80 & 70 & 60 & 50 & 40 & 30 & 20 & 10 & 0 & -10 & -:\end{array}$ 
5k, ${ }^{1} \mathrm{H}$ NMR (300 MHz, $\mathrm{CDCl}_{3}$ ) (JC901A)

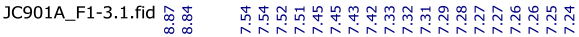<smiles>CC(C)c1nc(NC2CCCCC2)c2c(=O)n(Cc3ccccc3)c(=O)n(Cc3ccccc3)c2n1</smiles>

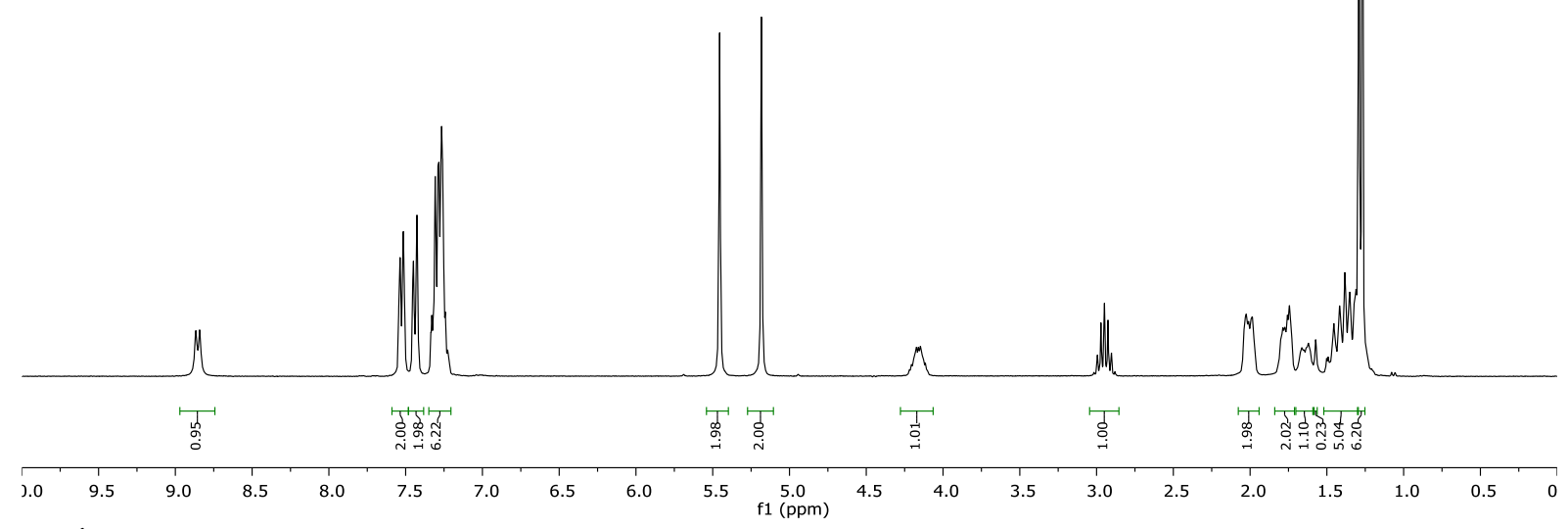

5k, ${ }^{1} \mathrm{H}$ NMR (125 MHz, $\mathrm{CDCl}_{3}$ ) (JC901A)

JC901A.11.fid (125)

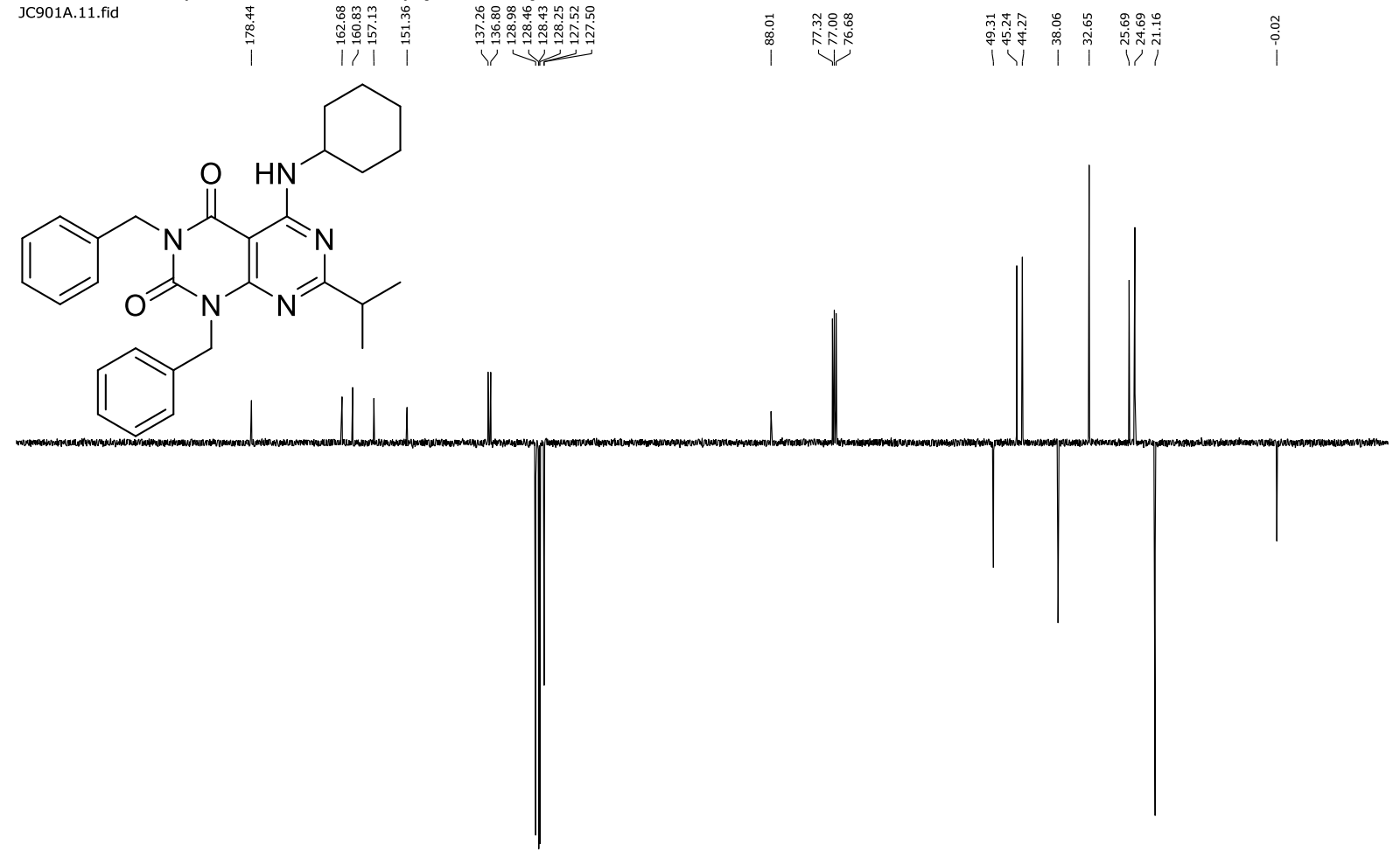

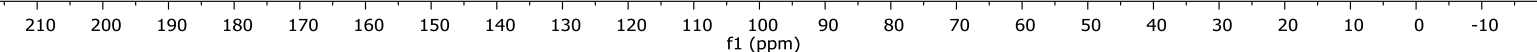


5m, ${ }^{1} \mathrm{H} \mathrm{NMR} \mathrm{(300} \mathrm{MHz,} \mathrm{CDCl}_{3}$ ) (JC901B)<smiles>Cc1cccc(C)c1Nc1nc(C(C)C)nc2c1c(=O)n(Cc1ccccc1)c(=O)n2Cc1ccccc1</smiles>

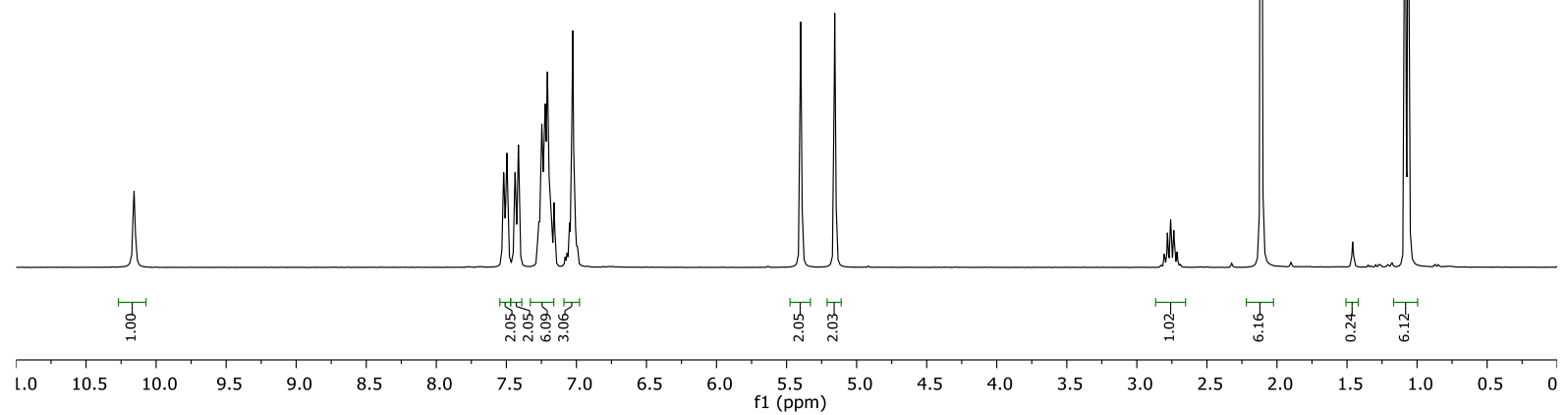

5m, ${ }^{1} \mathrm{H} \mathrm{NMR} \mathrm{(125} \mathrm{MHz,} \mathrm{CDCl} 3$ ) (JC901B)

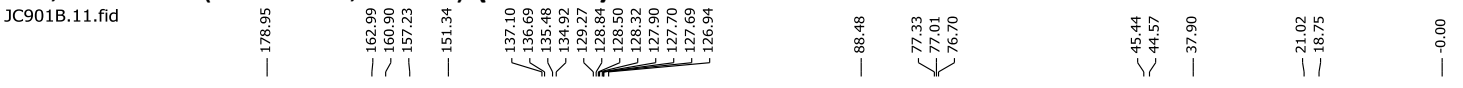

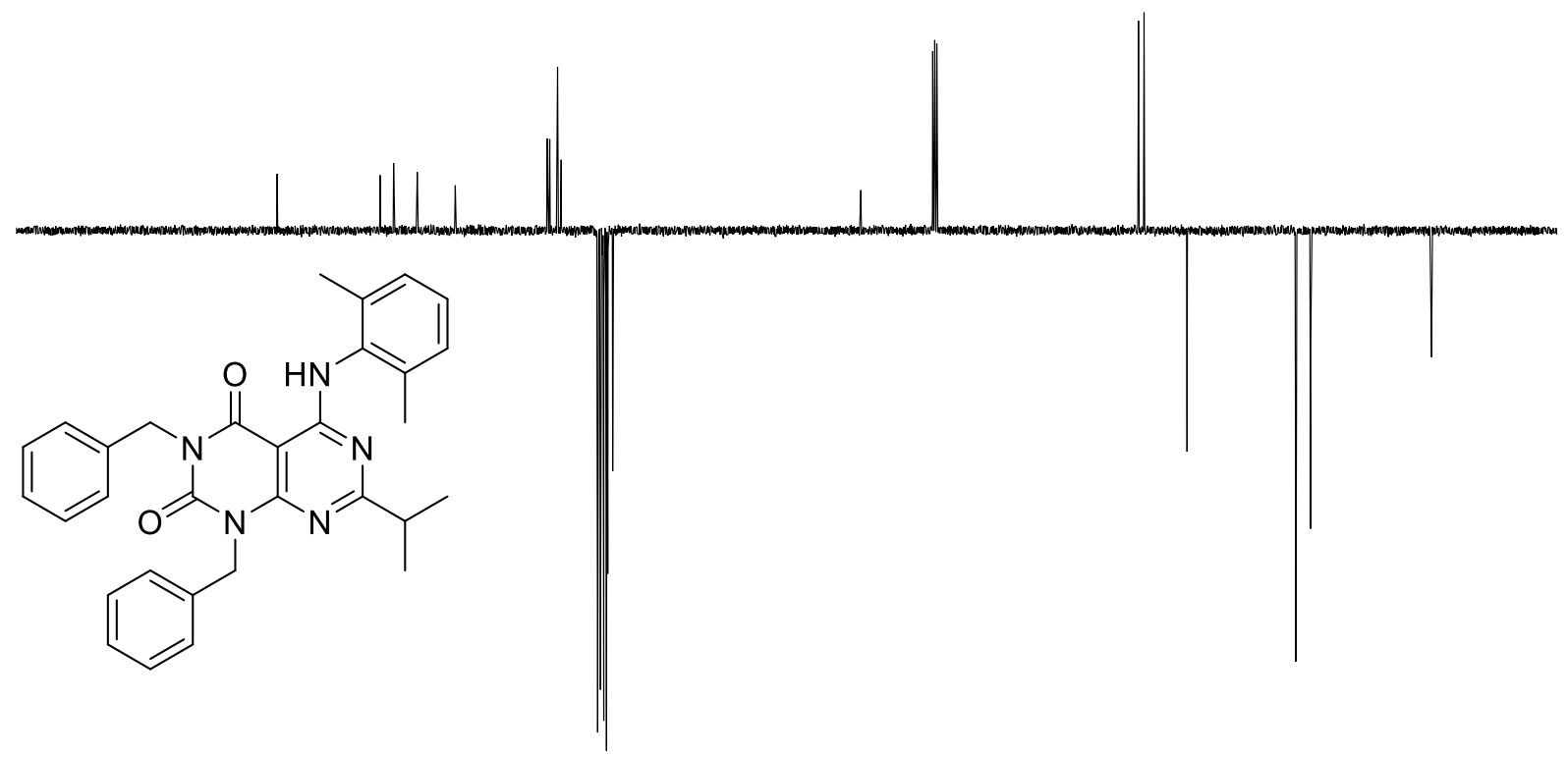

$\begin{array}{lllllllllllllllllllllllllll}210 & 200 & 190 & 180 & 170 & 160 & 150 & 140 & 130 & 120 & 110 & 100 & 90 & 80 & 70 & 60 & 50 & 40 & 30 & 20 & 10 & 0 & -10 & \end{array}$ 
5o, ${ }^{1} \mathrm{H}$ NMR (300 MHz, $\mathrm{CDCl}_{3}$ )

HLo70<smiles>CC(C)(C)Nc1nc(C(C)(C)C)nc2c1c(=O)n(Cc1ccccc1)c(=O)n2Cc1ccccc1</smiles>

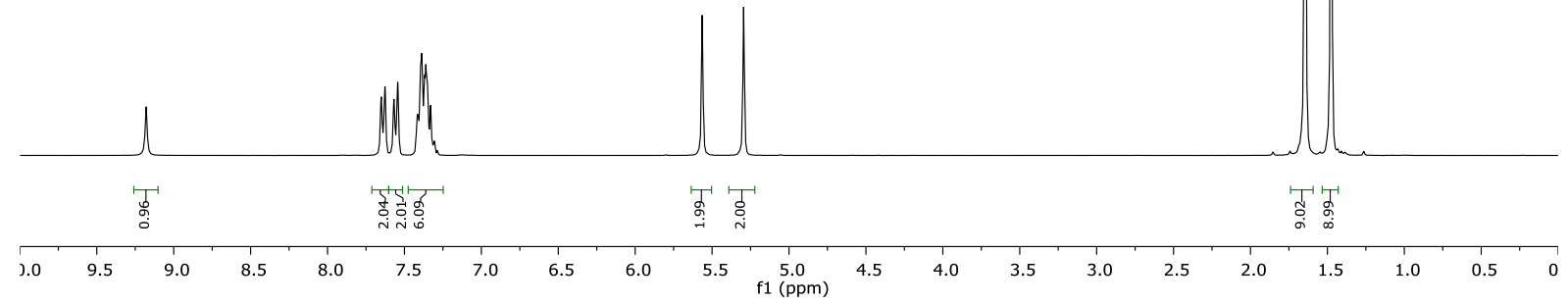

5o, ${ }^{1} \mathrm{H}$ NMR (126 MHz, $\mathrm{CDCl}_{3}$ )

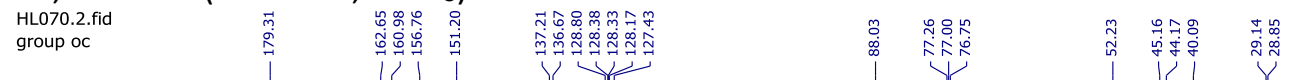

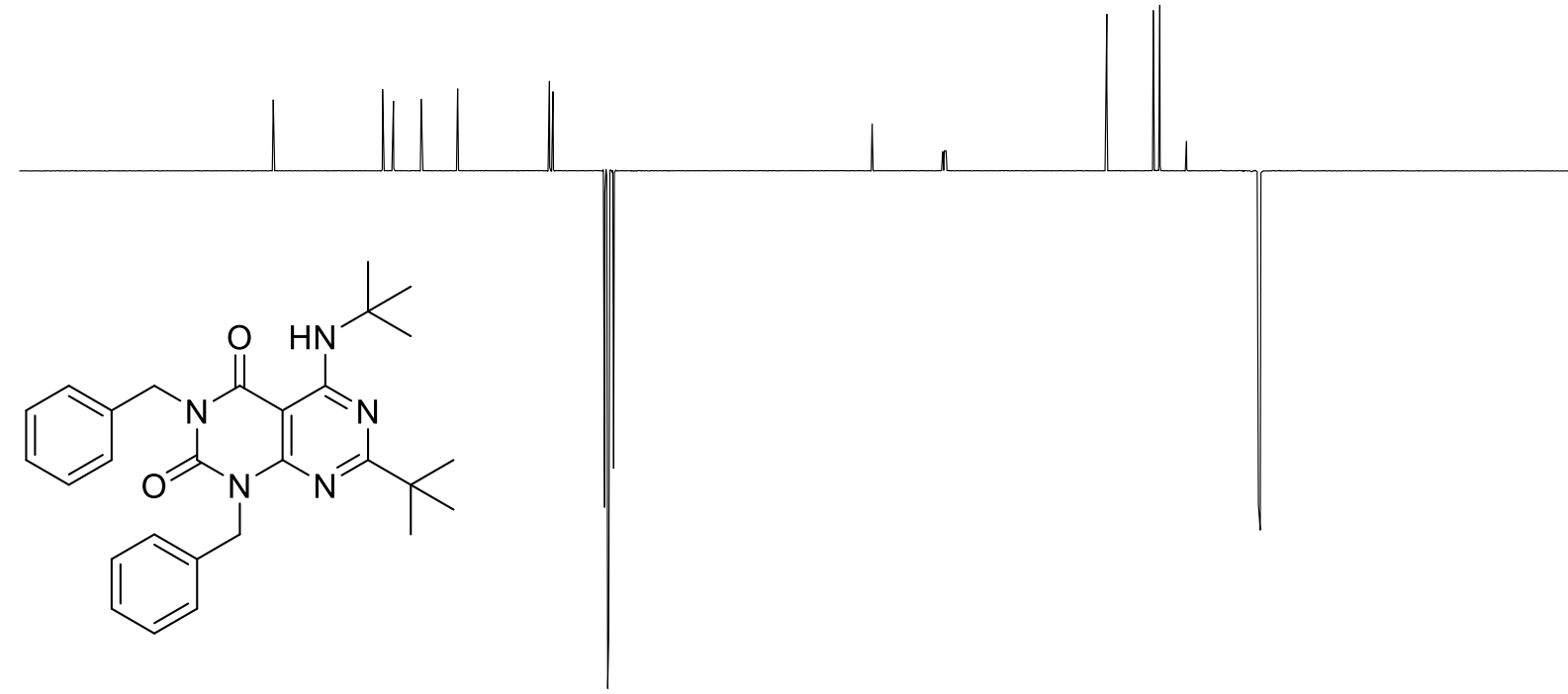

$\begin{array}{lllllllllllllllllllllll}210 & 200 & 190 & 180 & 170 & 160 & 150 & 140 & 130 & 120 & 110 & \begin{array}{c}100 \\ \mathrm{f} 1(\mathrm{ppm})\end{array} & 90 & 80 & 70 & 60 & 50 & 40 & 30 & 20 & 10 & 0 & -10\end{array}$ 
5p, ${ }^{1} \mathrm{H}$ NMR (300 MHz, $\mathrm{CDCl}_{3}$ )

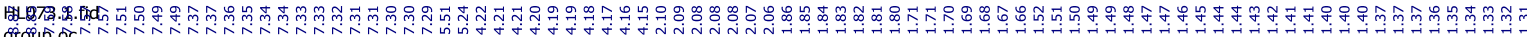<smiles>CC(C)(C)c1nc(NC2CCCCC2)c2c(=O)n(Cc3ccccc3)c(=O)n(Cc3ccccc3)c2n1</smiles>

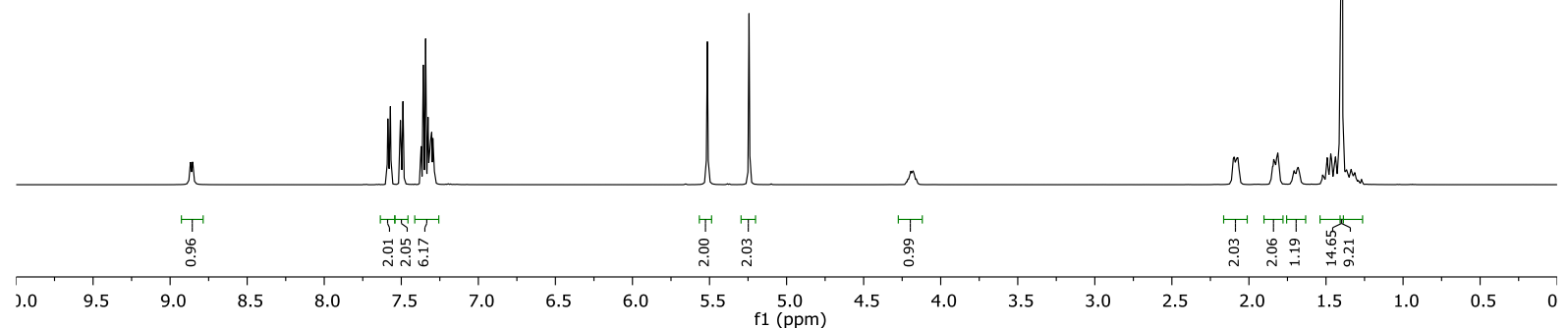

5p, ${ }^{1} \mathrm{H}$ NMR (126 MHz, $\mathrm{CDCl}_{3}$ )

$\begin{array}{llll}\text { HL073.2.fid } & \text { group oc } & \text { m } & 0\end{array}$

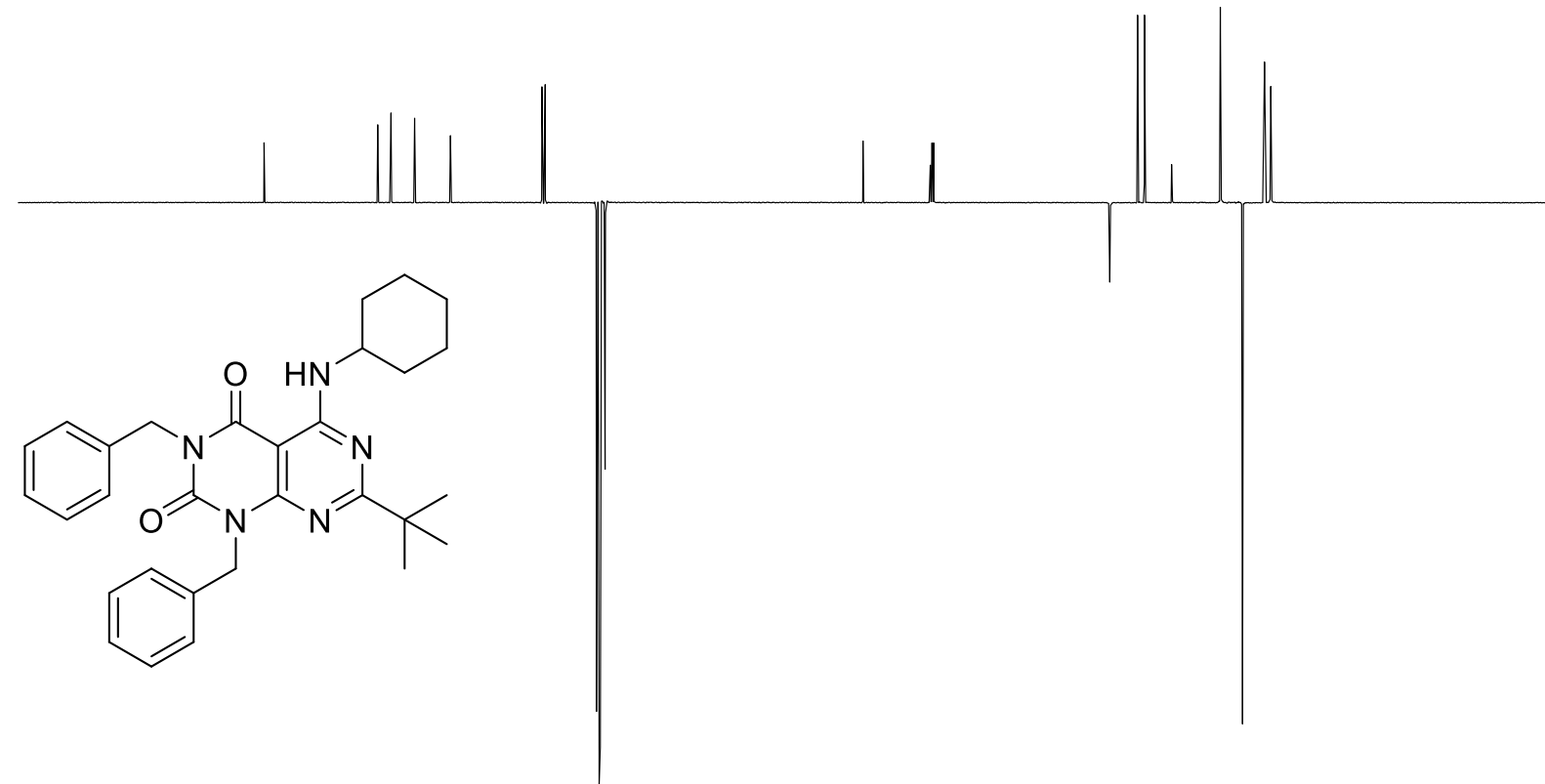

$\begin{array}{llllllllllllllllllllllllll}210 & 200 & 190 & 180 & 170 & 160 & 150 & 140 & 130 & 120 & 110 & 100 & 90 & 80 & 70 & 60 & 50 & 40 & 30 & 20 & 10 & 0 & -10\end{array}$ 
5q, ${ }^{1} \mathrm{H}$ NMR (300 MHz, $\mathrm{CDCl}_{3}$ )

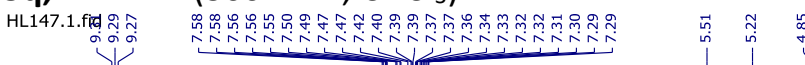

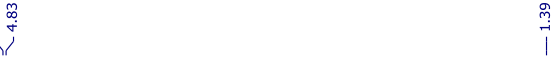<smiles>CC(C)(C)c1nc(NCc2ccccc2)c2c(=O)n(Cc3ccccc3)c(=O)n(Cc3ccccc3)c2n1</smiles>

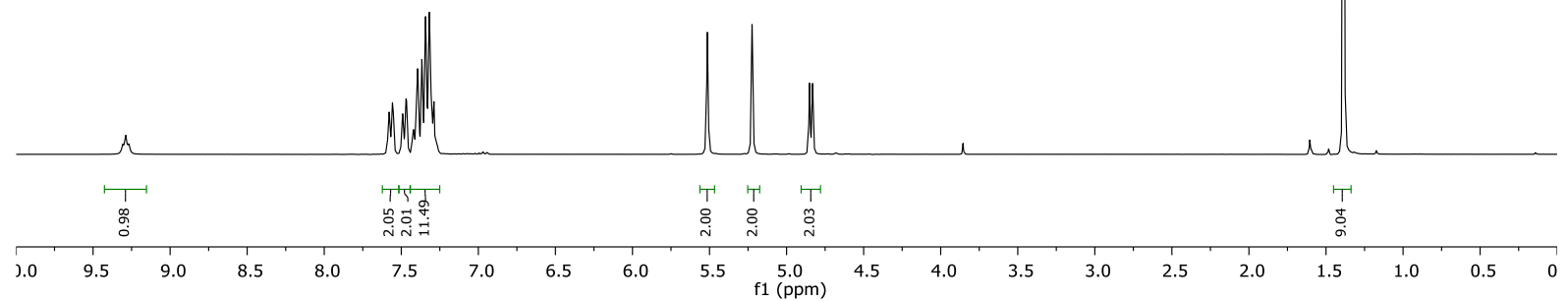

5q, ${ }^{1} \mathrm{H}$ NMR (126 MHz, $\mathrm{CDCl}_{3}$ )

HL147.1.fid

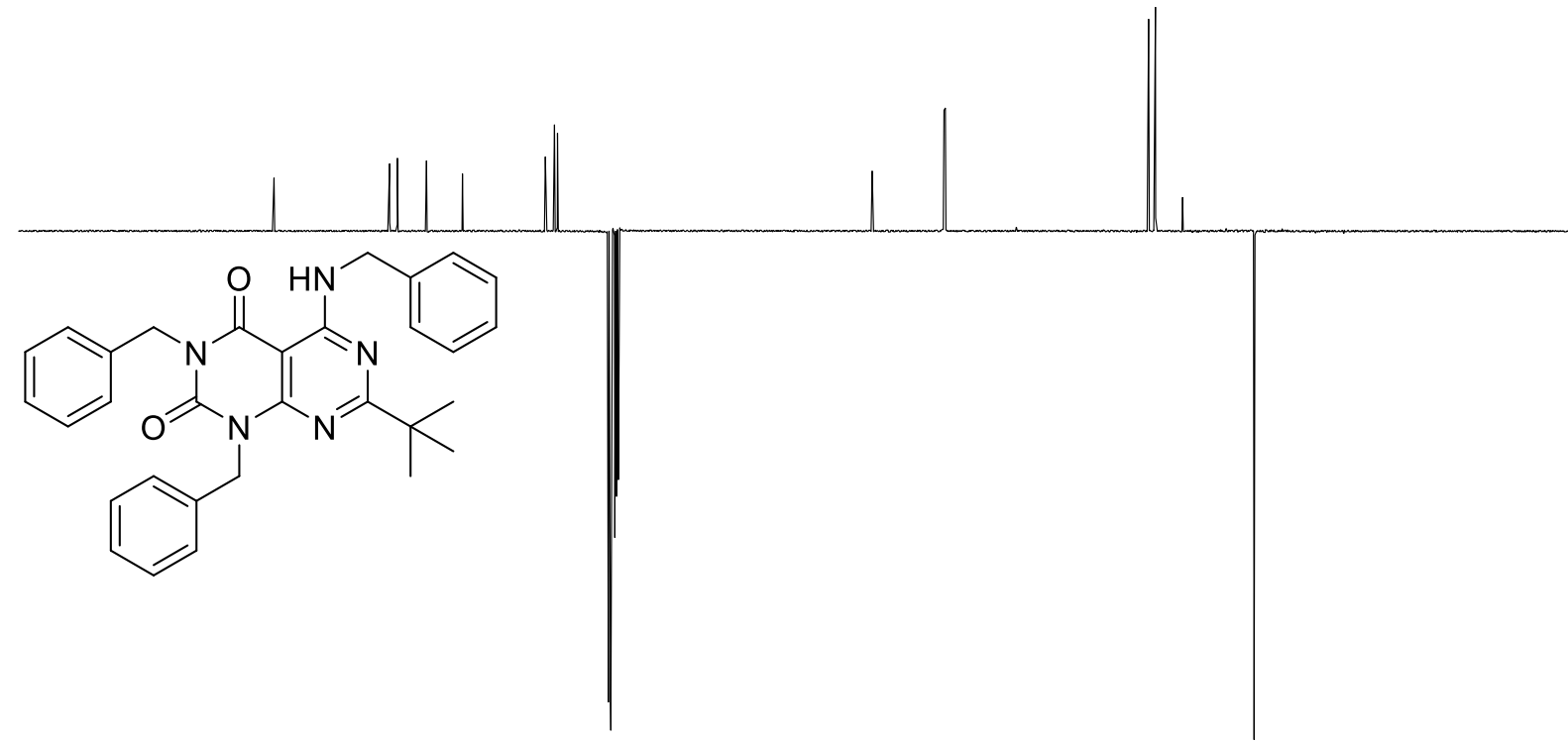

$\begin{array}{llllllllllllllllllllllllllll}210 & 200 & 190 & 180 & 170 & 160 & 150 & 140 & 130 & 120 & 110 & 100 & 90 & 80 & 70 & 60 & 50 & 40 & 30 & 20 & 10 & 0 & -10 & -i\end{array}$ 
5r, ${ }^{1} \mathrm{H}$ NMR (300 MHz, $\mathrm{CDCl}_{3}$ )

HL076.

|<smiles>Cc1cccc(C)c1Nc1nc(C(C)(C)C)nc2c1c(=O)n(Cc1ccccc1)c(=O)n2Cc1ccccc1</smiles>

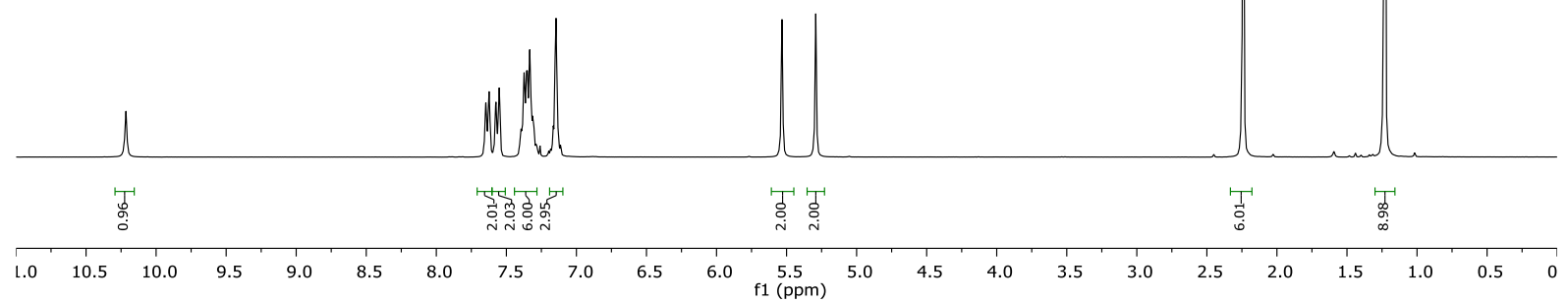

$5 r,{ }^{1} \mathrm{H}$ NMR (126 MHz, $\left.\mathrm{CDCl}_{3}\right)$

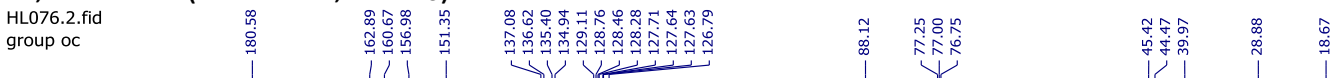

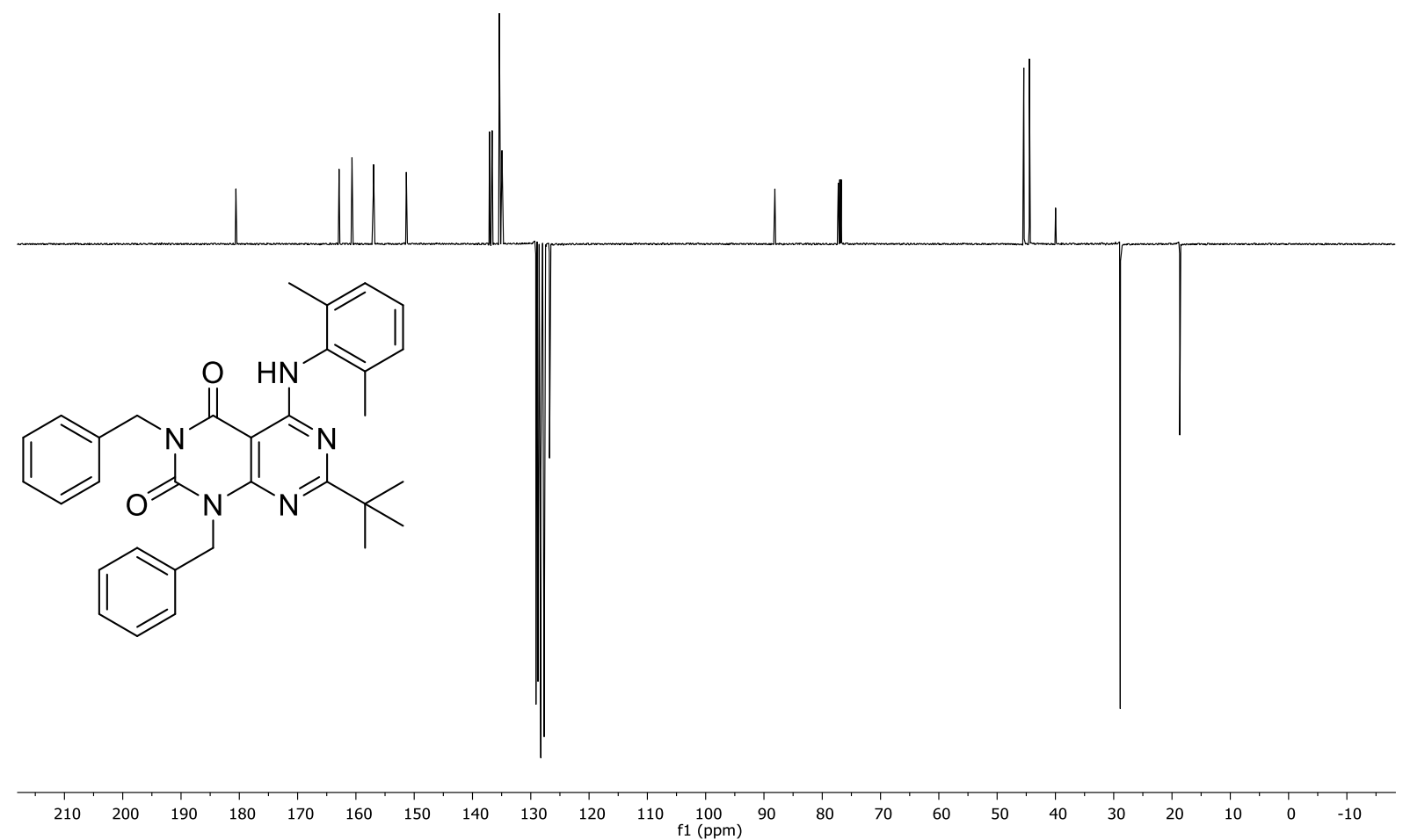


5s, ${ }^{1} \mathrm{H}$ NMR $\left(300 \mathrm{MHz}, \mathrm{CDCl}_{3}\right)$

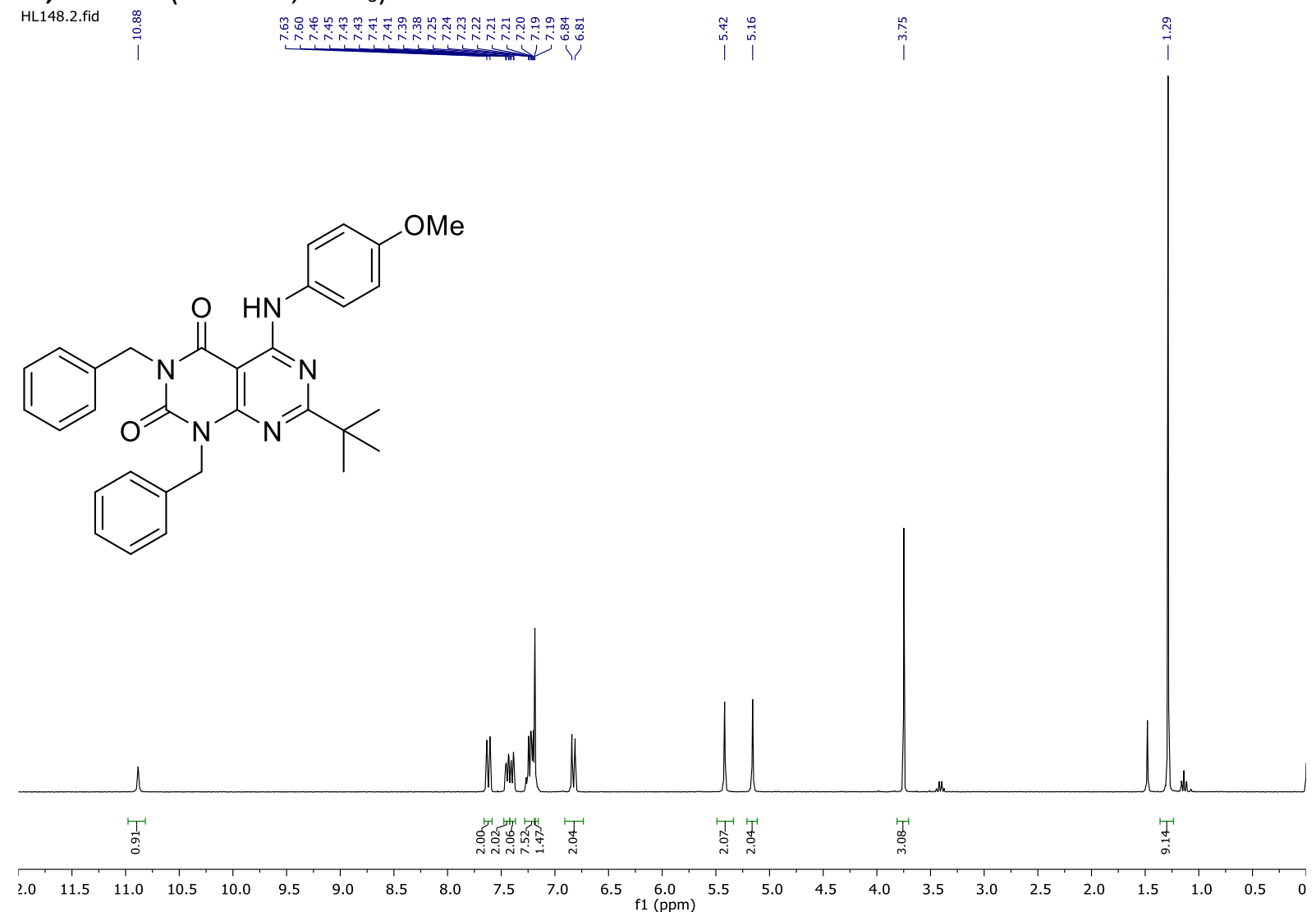

5s, ${ }^{1} \mathrm{H}$ NMR (126 MHz, $\mathrm{CDCl}_{3}$ )

HL148.1.fid

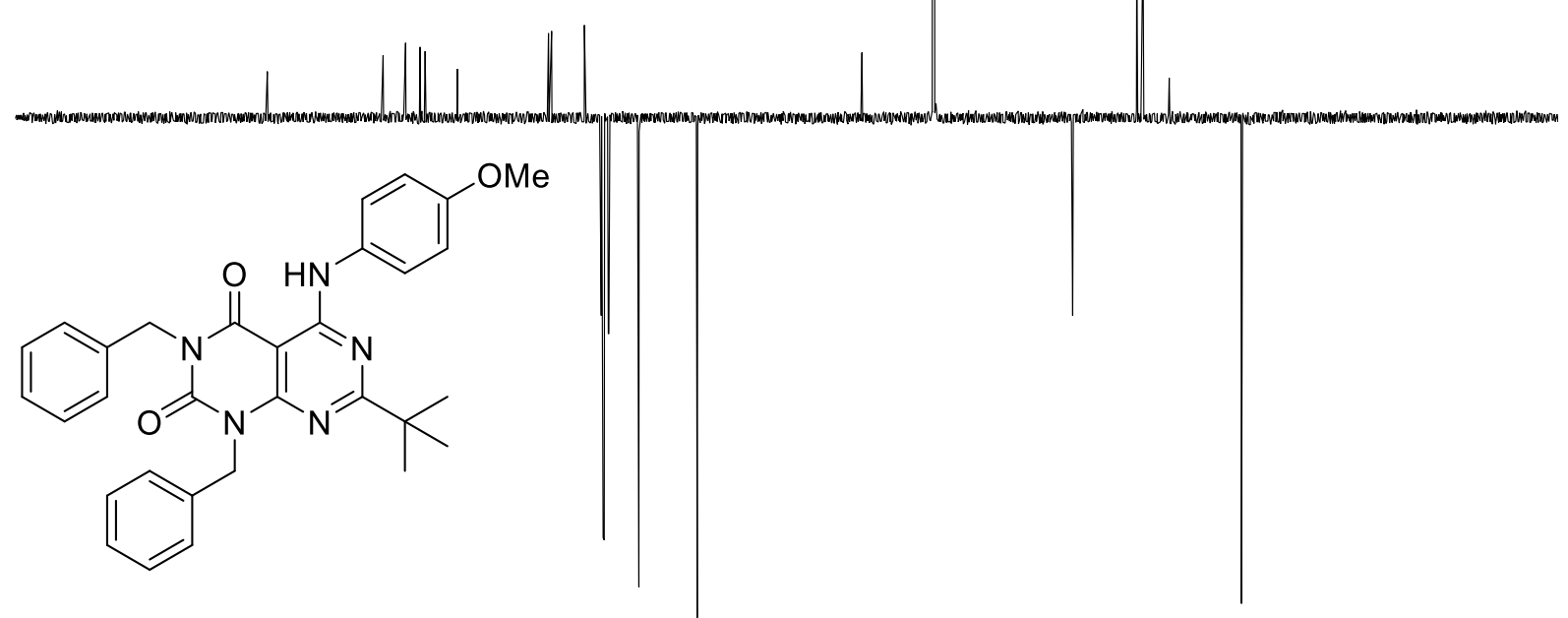

\begin{tabular}{rllllllllllllllllllllllllll}
\hline 20 & 210 & 200 & 190 & 180 & 170 & 160 & 150 & 140 & 130 & 120 & 110 & 100 & 90 & 80 & 70 & 60 & 50 & 40 & 30 & 20 & 10 & 0 & -10 & $-:$
\end{tabular} 
5t, ${ }^{1} \mathrm{H}$ NMR $\left(300 \mathrm{MHz}, \mathrm{CDCl}_{3}\right)$

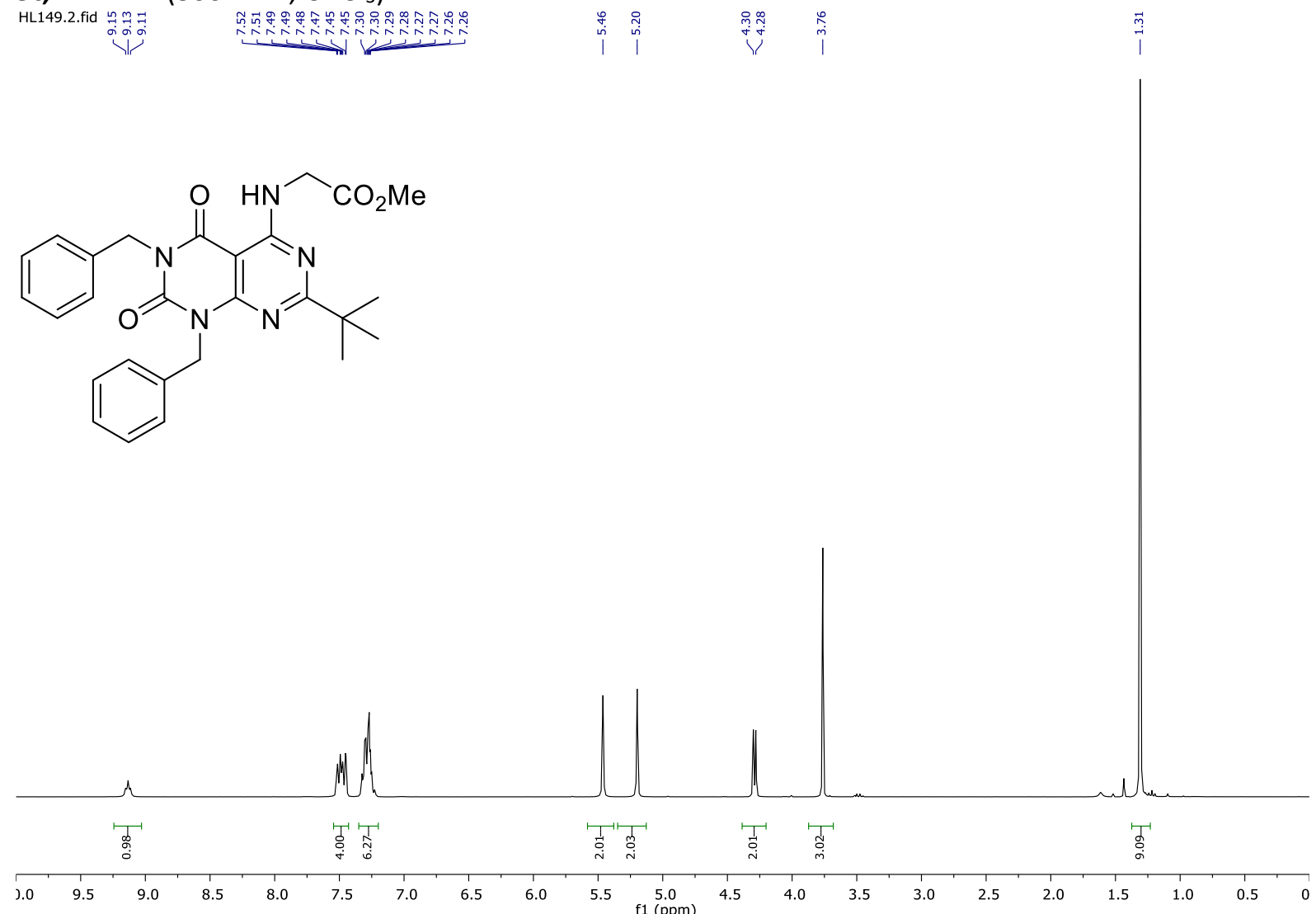

5t, ${ }^{1} \mathrm{H}$ NMR (126 MHz, $\mathrm{CDCl}_{3}$ )

HL149.1.fid

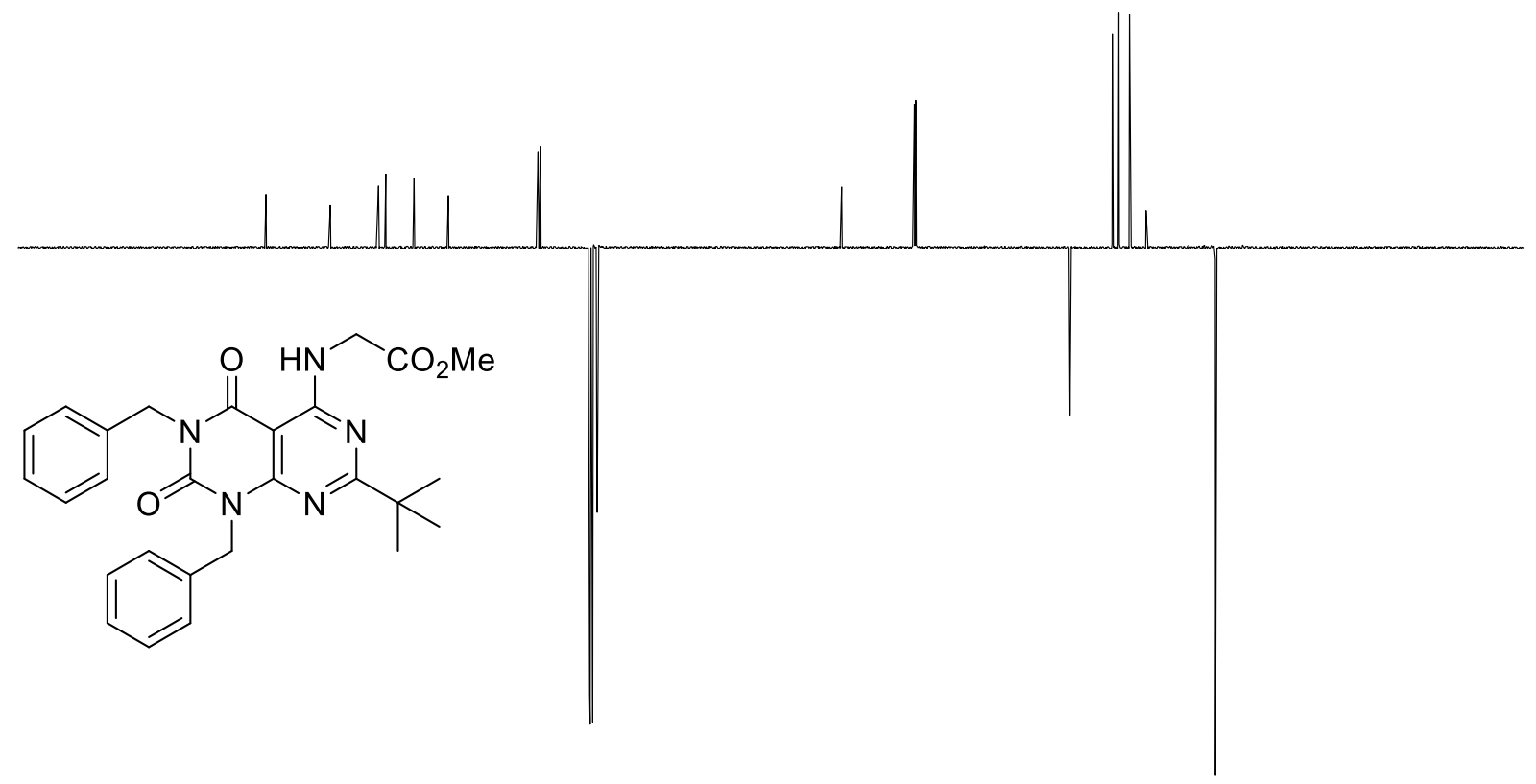

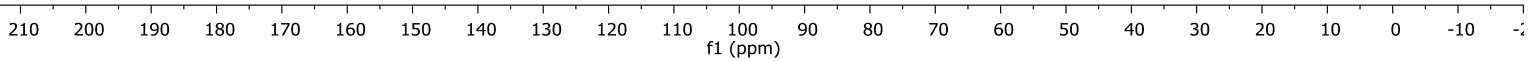


6a, $\left.{ }^{1} \mathrm{H} \mathrm{NMR} \mathrm{(300} \mathrm{MHz,} \mathrm{CDCl}_{3}\right)$ (JC755)

JC755_F3-6/1-10<smiles>Cn1c(=O)c2c(NC(C)(C)C)nc(-c3ccccc3)nc2n(C)c1=O</smiles>

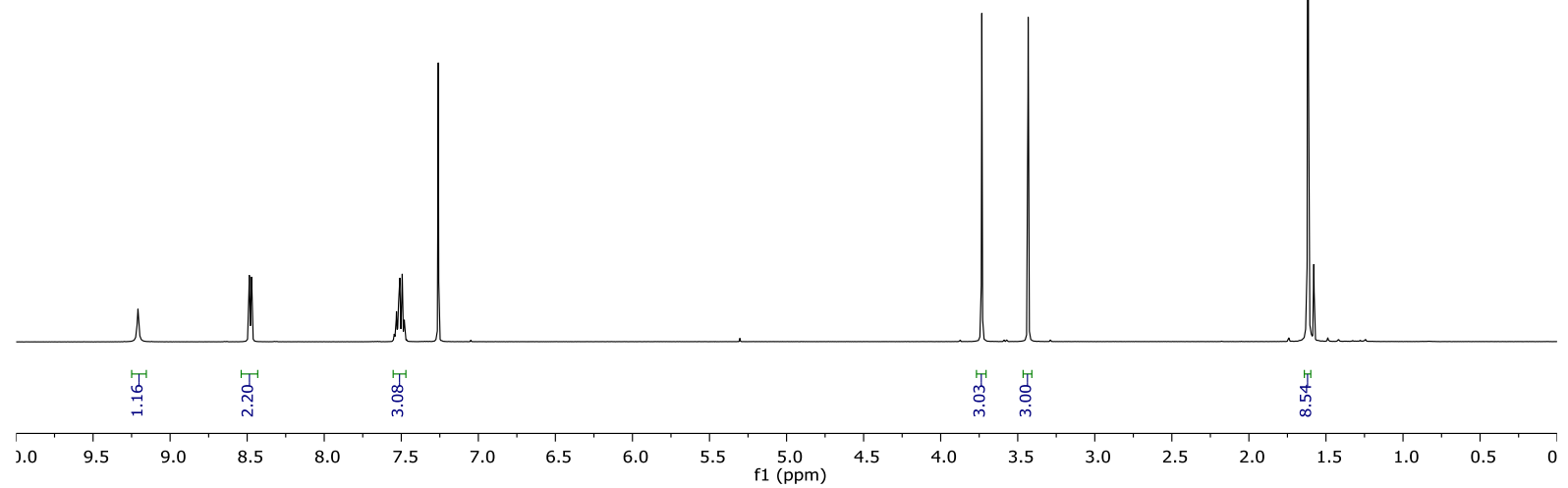

6a, ${ }^{1} \mathrm{H}$ NMR (125 MHz, $\left.\mathrm{CDCl}_{3}\right)$ (JC755)

JC755.11.fid

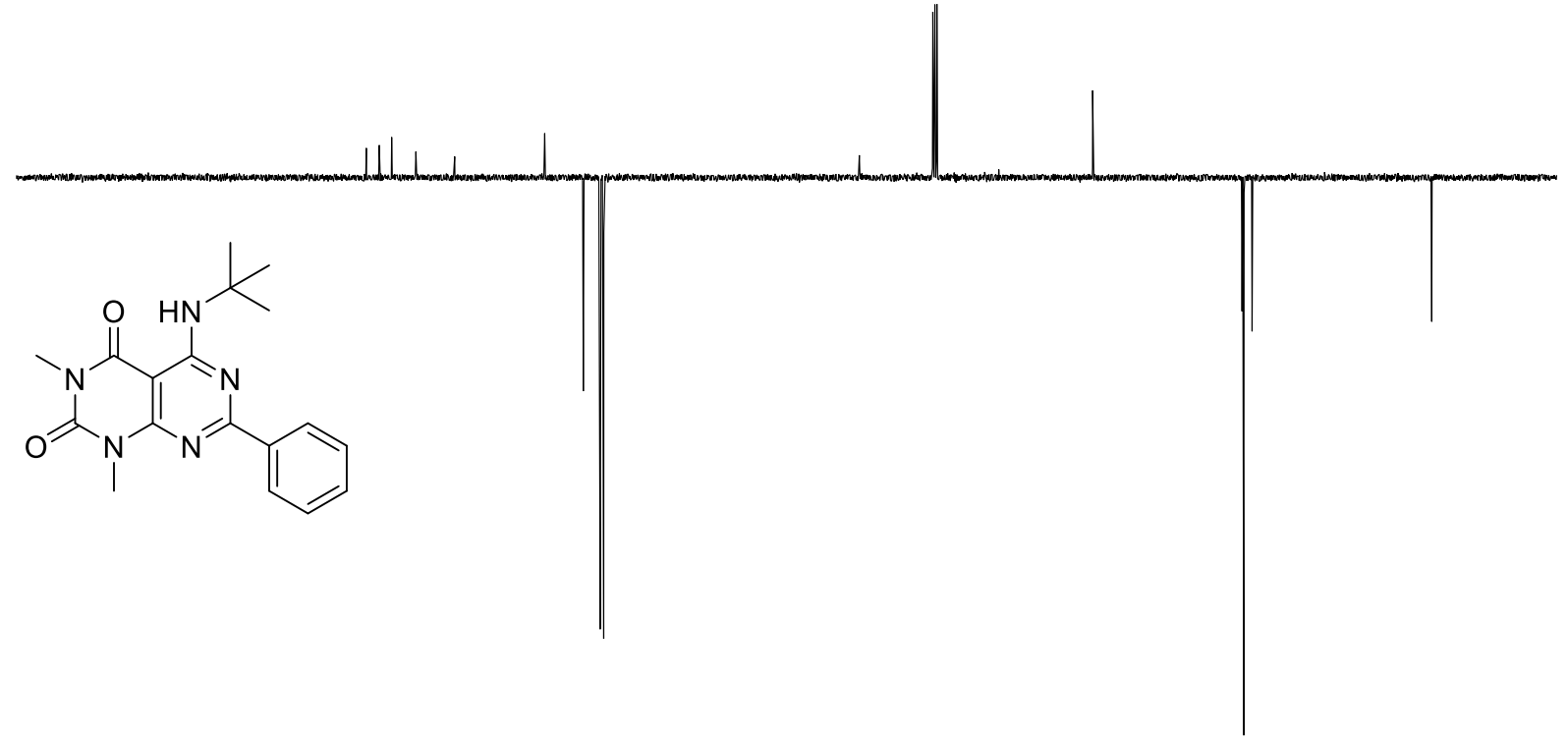

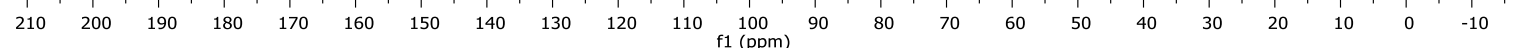


6b, ${ }^{1} \mathrm{H}$ NMR (400 MHz, $\mathrm{CDCl}_{3}$ ) (JC733)

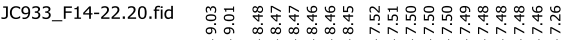<smiles>Cn1c(=O)c2c(NC3CCCCC3)nc(-c3ccccc3)nc2n(C)c1=O</smiles>

M.Mm Mu

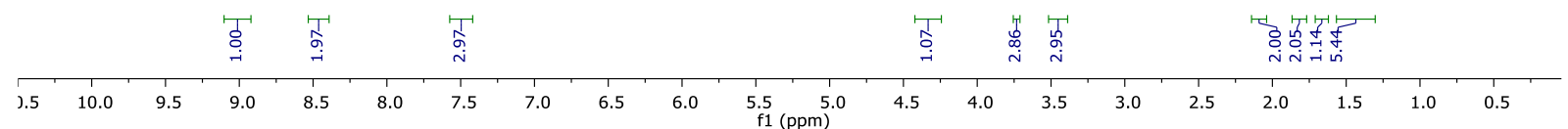

6b, ${ }^{1} \mathrm{H}$ NMR (126 MHz, $\mathrm{CDCl}_{3}$ ) (JC733)

JC933_F14-22.21.fid<smiles>Cn1c(=O)c2c(NC3CCCCC3)nc(-c3ccccc3)nc2n(C)c1=O</smiles>

$\begin{array}{lllllllllll}210 & 200 & 190 & 180 & 170 & 160 & 150 & 140 & 130 & 120 & \begin{array}{c}110 \\ \mathrm{f} 1(\mathrm{ppm})\end{array}\end{array}$

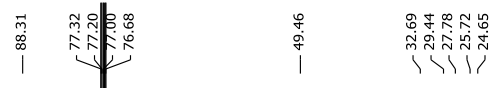


6c, ${ }^{1} \mathrm{H}$ NMR (300 MHz, $\mathrm{CDCl}_{3}$ )

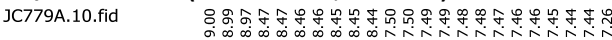<smiles>CCCCNc1nc(-c2ccccc2)nc2c1c(=O)n(C)c(=O)n2C</smiles>

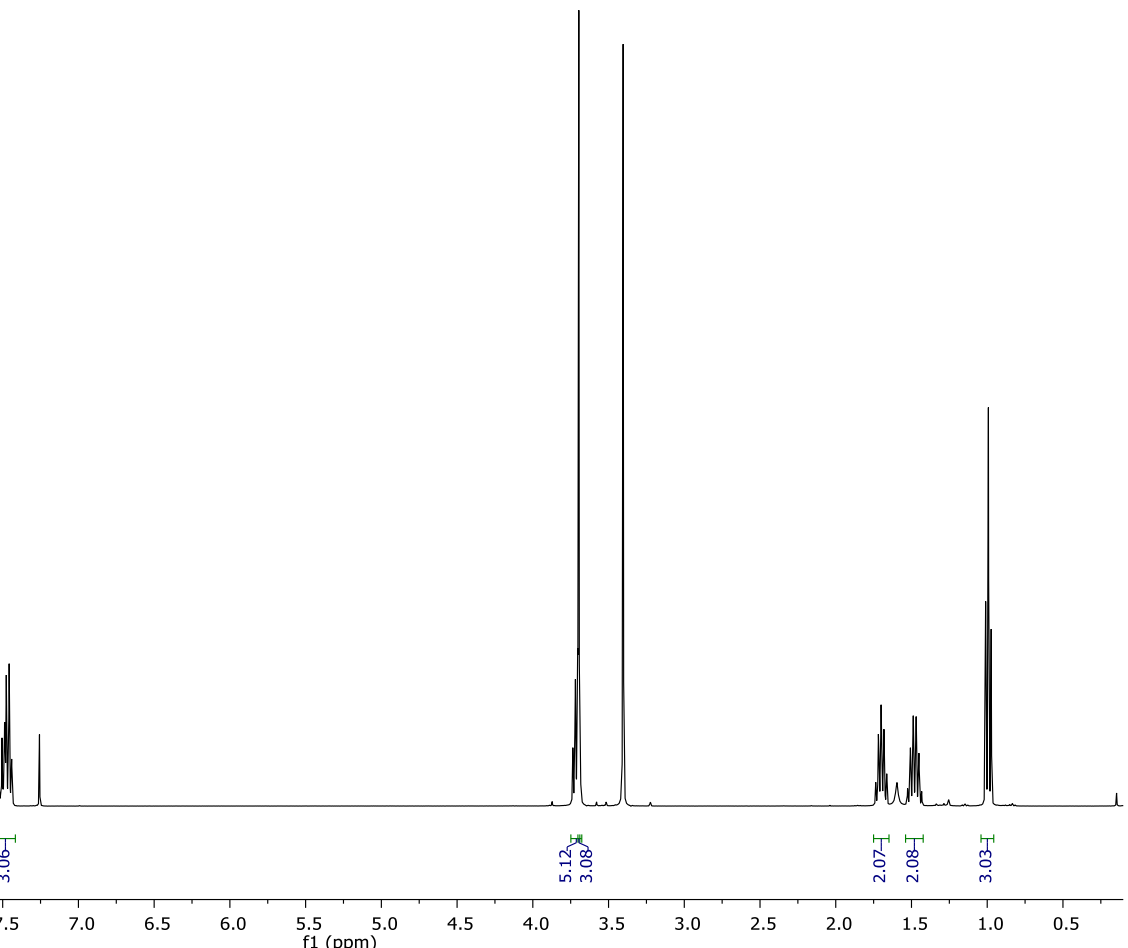

6c, ${ }^{1} \mathrm{H}$ NMR (126 MHz, $\left.\mathrm{CDCl}_{3}\right)$

JC779A.11.fid

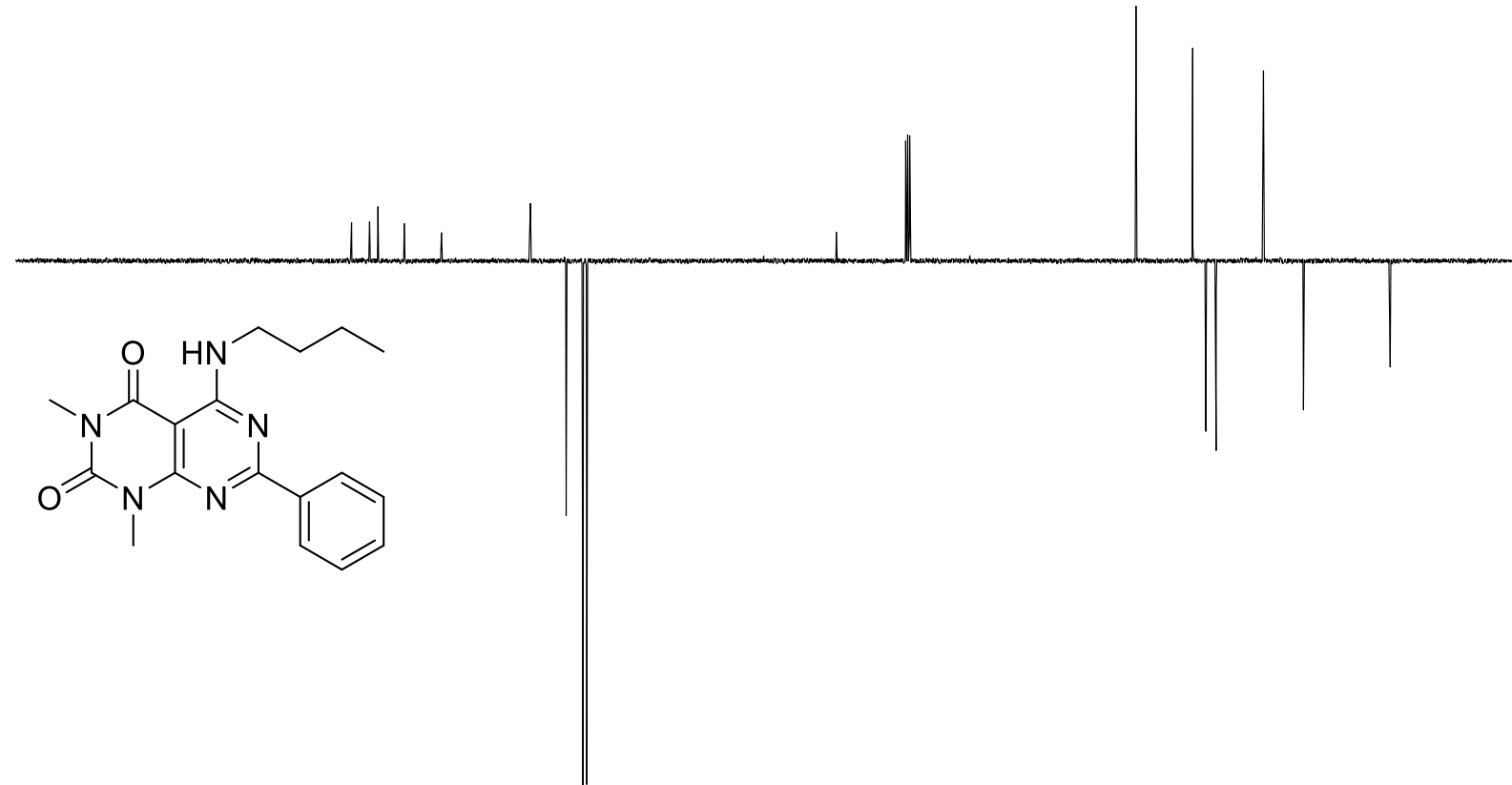

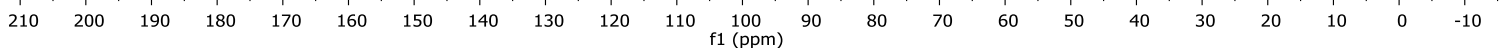


6d, ${ }^{1} \mathrm{H}$ NMR (300 MHz, $\mathrm{CDCl}_{3}$ ) (JC784B)

JC784B_F3-28.1.fid<smiles>Cn1c(=O)c2c(NCCN3CCOCC3)nc(-c3ccccc3)nc2n(C)c1=O</smiles>
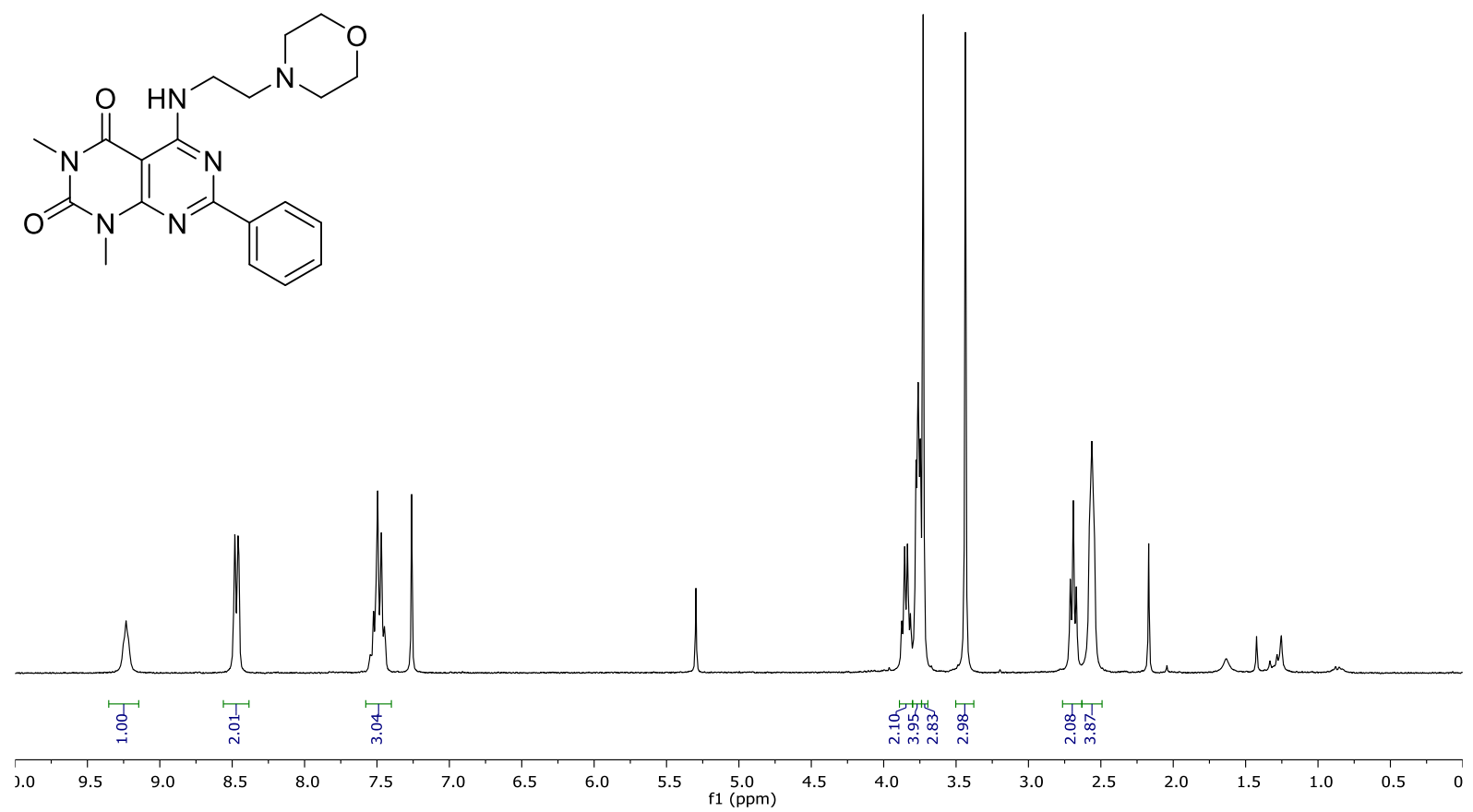

6d, ${ }^{1} \mathrm{H}$ NMR (125 MHz, $\mathrm{CDCl}_{3}$ ) (JC784B)

JC784B.11.fid<smiles>Cn1c(=O)c2c(NCCN3CCOCC3)nc(-c3ccccc3)nc2n(C)c1=O</smiles>
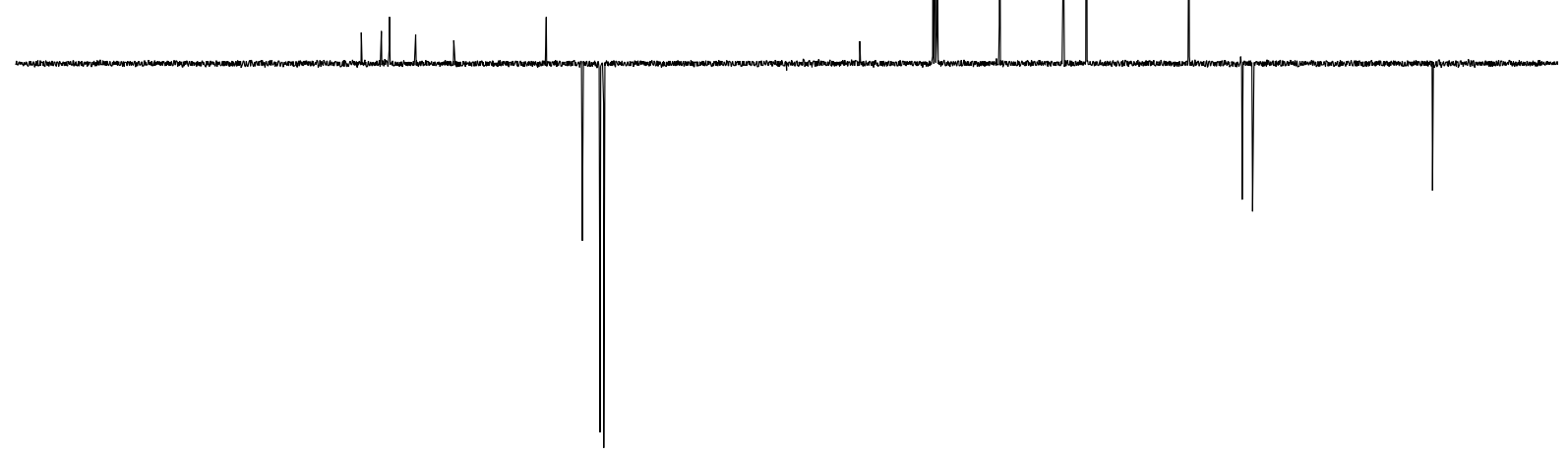

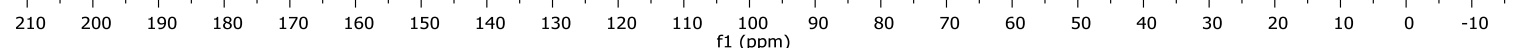


6e, ${ }^{1} \mathrm{H}$ NMR (300 MHz, CDCl 3 ) (JC959)

JC959_pp.3.fides
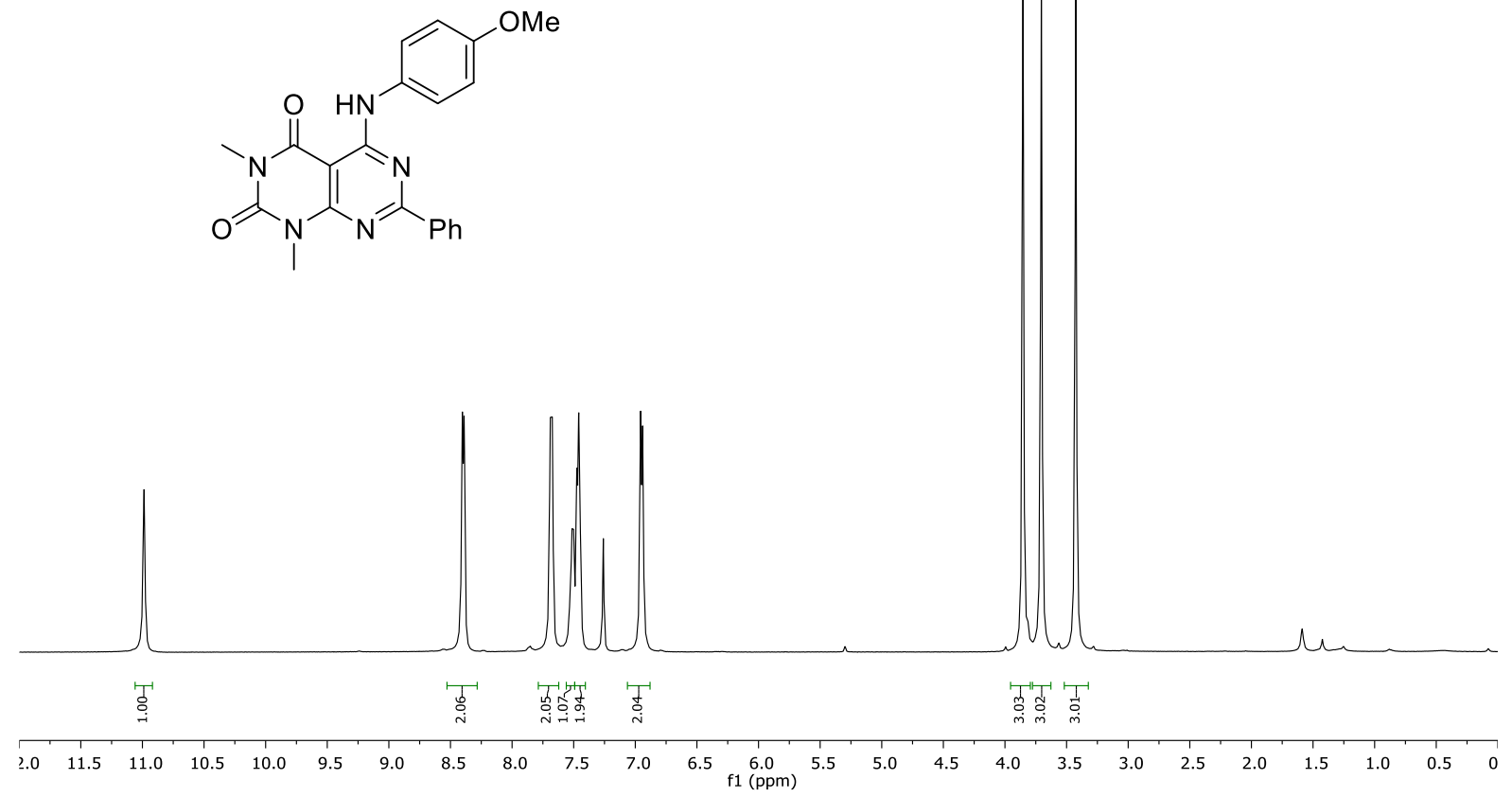

6e, ${ }^{1} \mathrm{H}$ NMR (126 MHz, $\mathrm{CDCl}_{3}$ ) 내요의

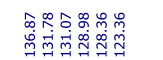

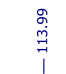<smiles>COc1ccc(Nc2nc(-c3ccccc3)nc3c2c(=O)n(C)c(=O)n3C)cc1</smiles>

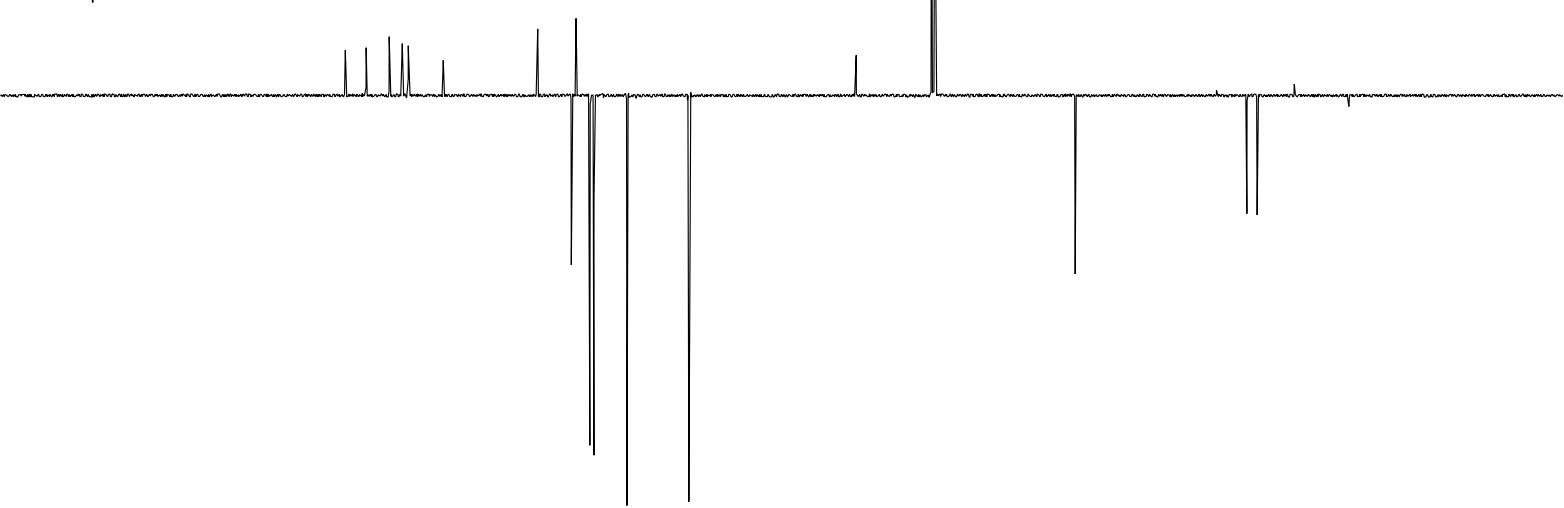

$\begin{array}{llllllllllllllllllllllll}210 & 200 & 190 & 180 & 170 & 160 & 150 & 140 & 130 & 120 & 110 & \begin{array}{c}100 \\ \mathrm{f} 1(\mathrm{ppm})\end{array} & 90 & 80 & 70 & 60 & 50 & 40 & 30 & 20 & 10 & 0 & -10\end{array}$ 
7, ${ }^{1} \mathrm{H}$ NMR (300 MHz, DMSO-d6)

总景

侽

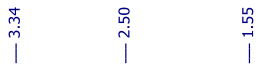<smiles>CC(C)(C)Nc1nc(-c2ccccc2)nc2[nH]c(=O)[nH]c(=O)c12</smiles>

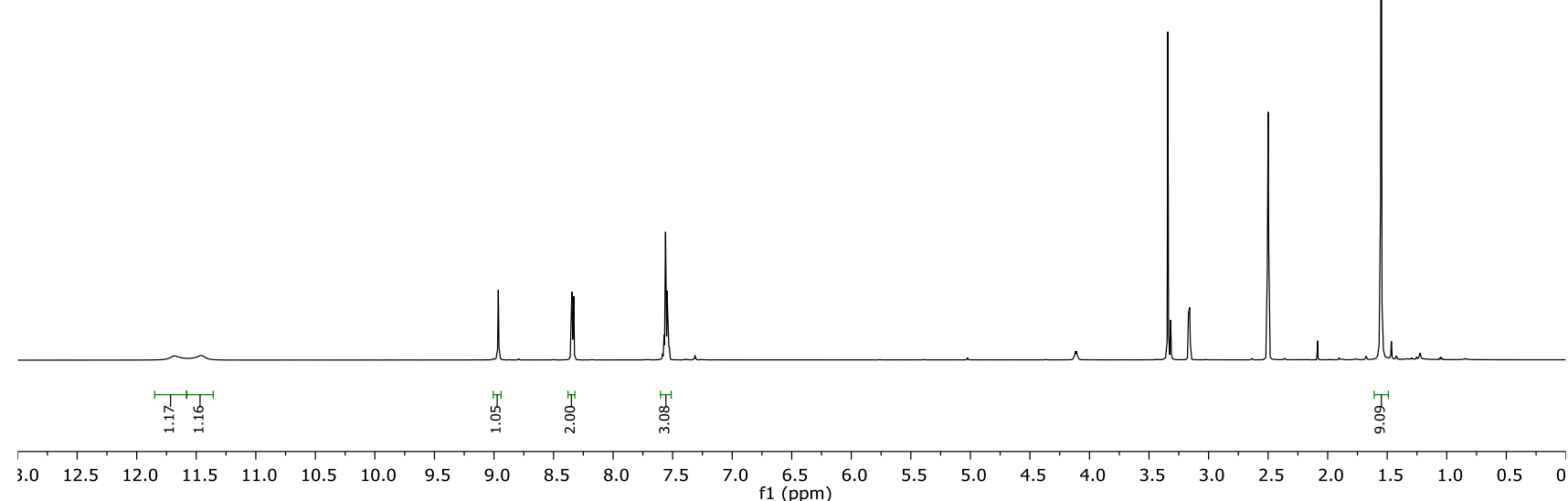

7, ${ }^{1} \mathrm{H}$ NMR (125 MHz, DMSO-d6)

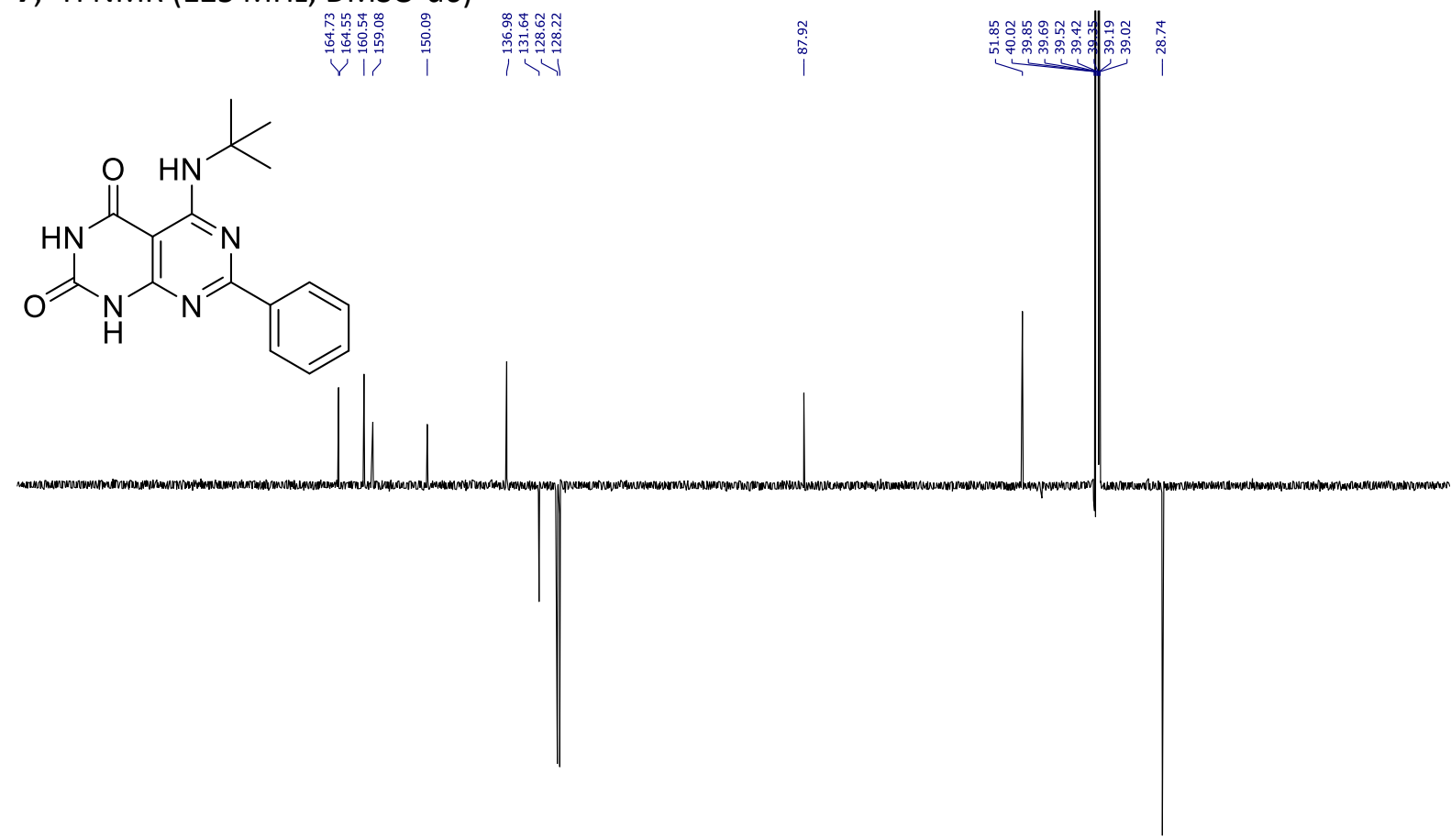

$\begin{array}{lllllllllllllllllllllll}210 & 200 & 190 & 180 & 170 & 160 & 150 & 140 & 130 & 120 & 110 & 100 & 90 & 80 & 70 & 60 & 50 & 40 & 30 & 20 & 10 & 0 & -10\end{array}$ 
8, ${ }^{1} \mathrm{H}$ NMR (300 MHz, DMSO-d6)

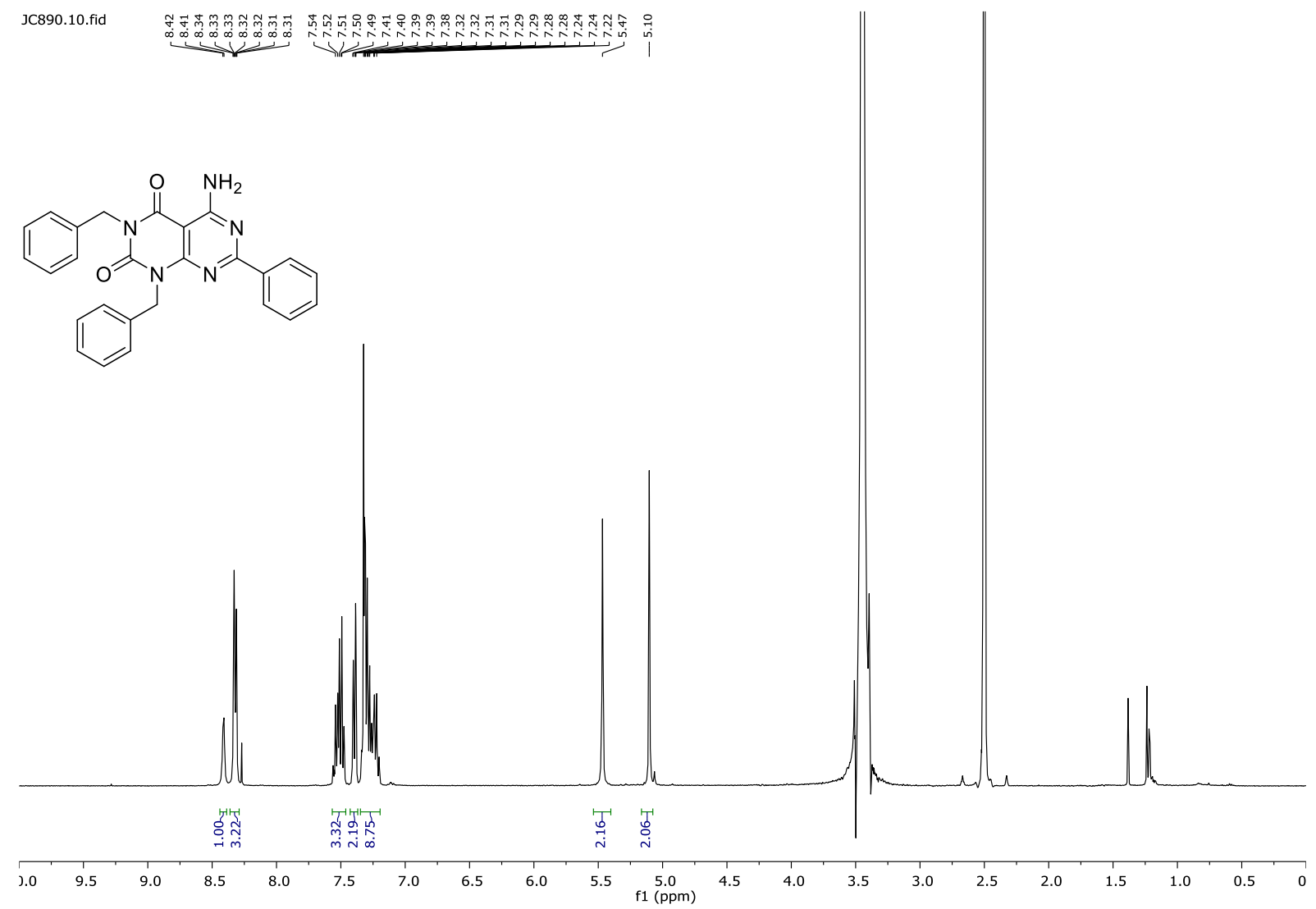

8, ${ }^{1} \mathrm{H}$ NMR (126 MHz, DMSO-d6)

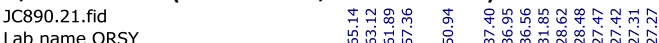

Lab name ORSY

jcollet 44! I) । I
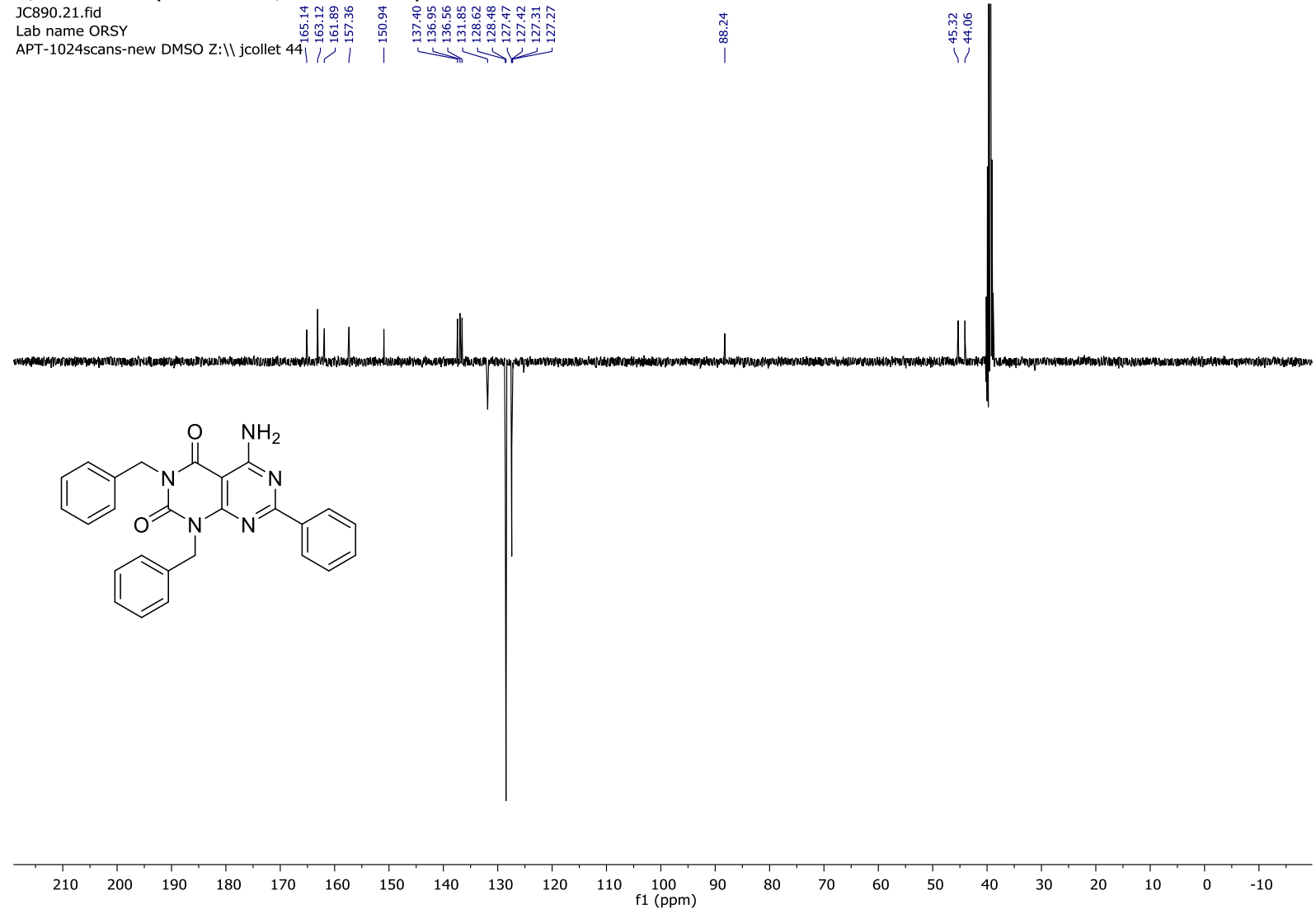


\section{References}

[1] Y. Wang, H. Wang, J. Peng, Q. Zhu, Org. Lett. 2011, 13, 4604-4607.

[2] D. Prat, A. Wells, J. Hayler, H. Sneddon, C. R. McElroy, S. Abou-Shehada, P. J. Dunn, Green Chem. 2016, 18, 288-296.

[3] a) T.-H. Zhu, S.-Y. Wang, T.-Q. Wei, S.-J. Ji, Adv. Synth. Catal. 2015, 357, 823-828. b) Z.-Q. Zhu, T.-T. Wang, P. Bai, Z.-Z. Huang, Org. Biomol. Chem. 2014, 12, 5839-5842.

[4] B. Morel, P. Franck, J. Bidange, S. Sergeyev, D. A. Smith, J. D. Moseley, B. U. W. Maes, ChemSusChem 2017, 10, 624-628. 